\title{
OPTIMIZING MACRO AND MICRO BASE STATIONS \\ LOCATIONS AND USER ASSOCIATIONS IN HETEROGENEOUS WIRELESS NETWORKS
}

\author{
by \\ Noha HASSAN \\ B.SC Faculty of Engineering, Alexandria University, Egypt, 2002 \\ M.SC Faculty of Engineering, Alexandria University, Egypt, 2011
}

\begin{abstract}
A dissertation presented to Ryerson University
in partial fulfillment of the

requirements for the degree

of Doctor of Philosophy in the

program of Electrical and Computer Engineering
\end{abstract}

Toronto, Ontario, Canada, 2019

(C) Noha Hassan, 2019 


\section{Declaration of Authorship}

- I hereby declare that I am the sole author of this dissertation. This is a true copy of the dissertation, including any required final revisions, as accepted by my examiners.

- I authorize Ryerson University to lend this dissertation to other institutions or individuals for the purpose of scholarly research.

- I further authorize Ryerson University to reproduce this dissertation by photocopying or by other means, in total or in part, at the request of other institutions or individuals for the purpose of scholarly research.

- I understand that my dissertation may be made electronically available to the public 


\title{
Optimizing Macro and Micro Base Stations Locations and User Associations in Heterogeneous Wireless Networks
}

\author{
Electrical and Computer Engineering \\ Ryerson University
}

Noha Hassan, 2019 Doctor of Philosophy

\begin{abstract}
Heterogeneous Networks (HetNets) have gained the attraction of the communication industry recently, due to their promising ability to enhance the performance of future broadband Fifth Generation $(5 \mathrm{G})$ networks and are integral parts of $5 \mathrm{G}$ systems. They can be viewed in multi-dimensional space where, each slice represents a unique tier that has its own Base Station (BS)s and User Equipment (UE)s. Different tiers cooperate with each other for their mutual benefit. Data can be interactively exchanged among the tiers, and UEs have the flexibility to switch between the tiers. The cells in such a heterogeneous cellular networks have variable sizes, shapes, and coverage regions.

However, in HetNets with ultra dense BSs, the distance between them gets very small and, they suffer from very high levels of mutual interference. To improve the performance of HetNets, we have done multiple contributions in this dissertation. First, we have developed analytical derivations for optimizing pilot sequence length which is a very crucial factor in acquiring the Channel State Information (CSI) and the channel estimation process in general. Poisson Point Process (PPP) has been widely used to allocate BSs among various tiers so far. However, BS locations obtained using PPP approach may not be optimum to reduce interference. Therefore, in this dissertation, BSs locations are optimized to reduce the interference and improve the coverage and received signal power. Also, we have derived expressions for static UEs coverage probability and network energy efficiency in HetNets.

A proper UE association algorithm for HetNets is a great challenge. The classic max-Signal to Interference and Noise Ratio (SINR) or max-received signal strength (RSS) user association algorithms are inappropriate solutions for HetNets as UEs in this context will tend to connect to the Macro BS, which is the one with the highest signal power. A severe load imbalance and significant
\end{abstract}


inefficiency arises and impacts the performance.

The aforementioned algorithms tend to associate UEs to BSs with the best received signal power or signal quality. In HetNets, usually Macro BSs are the ones transmitting the strongest signals; hence most UEs tend to associate with the Macro BS leaving Micro BSs with less load. Also, the conventional max-SINR and max-RSS algorithms do not provide adequate results in multi-tier systems. We suggest two centralized algorithms, LSTD and RTLB, for an even UE association to provide fair load distribution. However RTLB outperforms LSTD in real time scenarios as it easily and quickly adapts to rapid network changes. Furthermore, we consider the mobility of nodes. We derive coverage probability for moving UEs considering both handover and no handover scenarios. Proposed algorithms are fast enough to associate the moving users to different Micro and Macro BSs appropriately in real time. Our algorithms are proved to be feasible and provide a path towards attainable future communication systems. 


\section{Acknowledgement}

Praise be to Allah, who has favoured us over many of his believing servants. I thank Allah for all his blessing for me and my family during all years of my life and for answering my prayers and granting me the strength and patience to complete this work.

I would like to express my sincere gratitude to my advisor Prof. Xavier Fernando for the continuous support of my Ph.D study and related research, for his patience, motivation, and immense knowledge. His guidance helped me in all the time of research and writing of this thesis. I could not have imagined having a better advisor and mentor for my Ph.D study.

Besides my advisor, I would like to thank the rest of my thesis committee for their insightful comments and encouragement. I really thank them for taking the time and effort to review my thesis.

I thank my fellow lab mates for the stimulating discussions, for working together, and for their wonderful collaboration. You supported me greatly and were always willing to help me.

Last but not the least, I would like to thank my family: my mom specially who encouraged me to apply for this degree. I thank her for all she did for me and our family. I thank her for believing in me, encouraging me to do my best. May her soul rest in peace and be granted the highest degrees of paradise.

I offer my regards and blessings to my husband for his patience, and for his constant encouragement. Thank you for giving so much time helping me. My sincere thanks go to my brother and sister for supporting me spiritually throughout writing this thesis and my life in general. 


\section{Contents}

Declaration

Abstract

Acknowledgement $\quad$ v

List of Figures $\quad$ ix

List of Tables $\quad$ xiii

List of Acronyms xiv

List of Symbols $\quad$ xviii

1 Introduction $\quad 1$

1.1 Homogeneous Single-tier Networks . . . . . . . . . . . . . . . . . . . . . 1

1.1.1 Research Challenges in Homogeneous Networks . . . . . . . . . . . . . . . . 3

1.2 Heterogeneous Multi-tier Networks . . . . . . . . . . . . . . . . . . . 4

1.2.1 Small cell Base Stations . . . . . . . . . . . . . . . . . . . . . 4

1.2.2 Heterogeneous Networks Benefits . . . . . . . . . . . . . . . 5

1.2.3 Comparison of Homogeneous and Heterogeneous Networks _ . . . . . . . . 6

1.2.4 Research Challenges in Heterogeneous Networks . . . . . . . . . . . . . . . 10

1.3 Massive MIMO and Pilot Contamination . . . . . . . . . . . . . . 10

1.3.1 Massive MIMO . . . . . . . . . . . . . . . . . . . . . 10

1.3 .2 Pilot contamination . . . . . . . . . . . . . . . . 11

1.4 Optimizing Base Stations Locations _ . . . . . . . . . . . . . . . . 13 
1.5 User Association . . . . . . . . . . . . . . . . . . . . 14

1.6 Chapter Summary . . . . . . . . . . . . . . . . . . 15

2 System Model and its Components 16

2.1 Chapter Overview . . . . . . . . . . . . . . . . . . 16

2.2 Uplink and Downlink Operations . . . . . . . . . . . . . . . . 16

2.2 .1 Uplink Operation . . . . . . . . . . . . . . . . . . . . 17

2.2 .2 Downlink Operation . . . . . . . . . . . . . . . . . . . 23

2.3 Channel Estimation Methods: (TDD or FDD?) . . . . . . . . . . . . . . . . . 24

2.3.1 Why did we use TDD? . . . . . . . . . . . . . 24

2.3 .2 Why did not we use FDD? . . . . . . . . . . . . . . . . 24

2.4 OFCDM Systems . . . . . . . . . . . . . . . . . . . . . . . 25

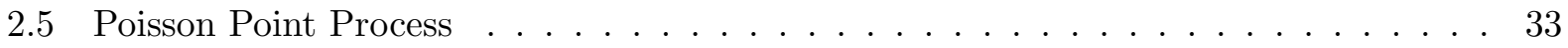

2.6 Description of System Model in this Dissertation . . . . . . . . . . . . . . . 33

2.7 Dissertation Motivation . . . . . . . . . . . . . . . 36

2.8 Dissertation Contributions . . . . . . . . . . . . . . . . . 37

2.9 Dissertation Outline . . . . . . . . . . . . . . . . . . . . 39

2.10 Chapter Summary . . . . . . . . . . . . . . . . . . . 41

3 Optimization of Pilot Sequence Length 42

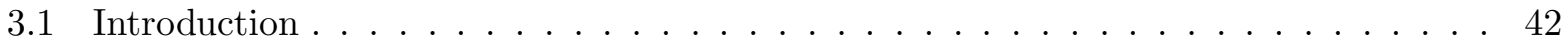

3.2 Related Work . . . . . . . . . . . . . . . . . . 43

3.3 Contributions and Organization . . . . . . . . . . . . . . . . 45

3.4 Pilot Length Problem Formulation . . . . . . . . . . . . . . . . . . . . 45

$3.4 .1 \quad$ Asymptotic Analysis . . . . . . . . . . . . . . . . . . . . . . . 49

3.5 Performance Analysis . . . . . . . . . . . . . . . . . . . 51

3.6 Chapter Summary . . . . . . . . . . . . . . . . . . . . 52

4 Optimization of Micro BSs Positions in HetNets 53

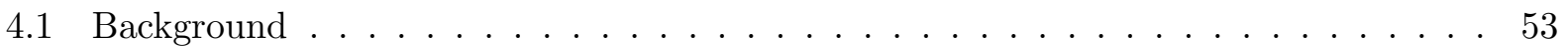

4.2 Related Work . . . . . . . . . . . . . . . . . . . . . 54 
4.3 Contributions and Organization . . . . . . . . . . . . . 58

4.4 Intermediate Distances Between Micro BSs . . . . . . . . . . . . . . . . . 58

4.5 Coverage Probability and Energy Efficiency Analysis in Two-tier Open Access HetNets 59

4.5.1 Coverage Probability Analysis . . . . . . . . . . . . . . . . . 59

4.5.2 Energy Efficiency Analysis . . . . . . . . . . . . . . . . . 64

4.6 Optimum Locations of Micro BSs. . . . . . . . . . . . . . . . . 67

4.6.1 Optimum Deployment of Micro BSs (OPLI Algorithm) . . . . . . . . . 68

4.6.2 Optimum Positions of Micro BSs to Maximize SINR . . . . . . . . . . . . 73

4.7 Performance Analysis . . . . . . . . . . . . . . . . . . 74

4.8 Chapter Summary . . . . . . . . . . . . . . . . . . 76

5 Optimizing Macro BSs Locations in HetNets for Eliminating Interference 82

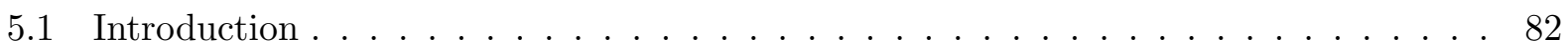

5.2 Related Work . . . . . . . . . . . . . . . . . . . . 83

5.3 Contributions and Organization . . . . . . . . . . . . . 84

5.4 Channel and Interference Models . . . . . . . . . . . . . . . . . . 84

5.5 Optimization of Macro BSs Locations in HetNets . . . . . . . . . . . . 86

5.5.1 Dummy Intersecting Circle (DIC) Problem Formulation . . . . . . . . . . 86

5.5.2 Modified Dummy Intersecting Circle (MDIC) Problem Formulation . . . . . . 89

5.6 Performance Analysis _ . . . . . . . . . . . . . . . . . . . 95

5.7 Sensitivity Analysis for Threshold Values . . . . . . . . . . . . . . 96

5.8 Chapter Summary . . . . . . . . . . . . . . . . . . . . 97

6 Load Distribution Standard Deviation (LSTD) User Association Algorithm 106

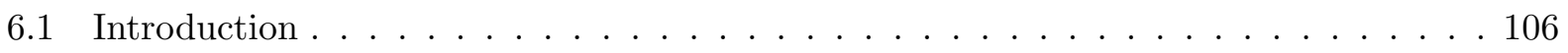

6.2 Related Work . . . . . . . . . . . . . . . . . . 106

6.3 Contributions and Organization . . . . . . . . . . . . . 107

6.4 Explanation of the Proposed LSTD Algorithm . . . . . . . . . . . . . . 107

6.5 Problem Formulation and Complexity Analysis . . . . . . . . . . . . . 108

6.6 Simulation Analysis f . . . . . . . . . . . . . . . . . 116

6.7 Chapter Summary . . . . . . . . . . . . . . . . . 117 
7 Real Time Load Balance (RTLB) UE Association Algorithm

7.1 Introduction . . . . . . . . . . . . . . . . . . . . . . 122

7.2 Related Work . . . . . . . . . . . . . . . . . . . 122

7.3 Contributions and Organization . . . . . . . . . . . . . . . . . 124

7.4 Explanation of RTLB User Association Algorithm . . . . . . . . . . . . . 124

7.5 Problem Formulation and Complexity Analysis . . . . . . . . . . . . . . . 126

7.6 UEs Mobility and Status Change Analysis . . . . . . . . . . . . . . . 131

7.7 Coverage Probability Considering Moving Users . . . . . . . . . . . . . . . . 132

7.8 Simulation Analysis . . . . . . . . . . . . . . . 136

7.9 Chapter Summary . . . . . . . . . . . . . . . . . . . . 139

8 Discussion and Future Work $\quad 145$

8.1 Objectives and Contributions . . . . . . . . . . . . . . 145

8.2 Future Developments . . . . . . . . . . . . . . . . . . . 146

8.3 Conclusions . . . . . . . . . . . . . . . . . . 147

$\begin{array}{ll}\text { Bibliography } & 147\end{array}$ 


\section{List of Figures}

1.1 An illustration of a PPP-based two-tier cellular network deployment. A $20 \mathrm{~km} \times 20$ $\mathrm{km}$ area, consisting of Femto-cells (crosses) and Macrocells (dots), is shown. . . . . . 7

1.2 NMSE and Sum-Rate capacity comparison f . . . . . . . . . . . . . . 9

2.1 Uplink and downlink operation. . . . . . . . . . . . . . . . . 17

2.2 BER for OFCDM System with Various Configurations. . . . . . . . . . . . . 30

2.3 OFDM-TDMA vs OFCDM-TDMA comparison. . . . . . . . . . . . . . 31

2.4 Poisson point process with $\lambda=55$, and thinning distance less than $200 \mathrm{~m}$. . . . . 32

2.5 An illustration of a PPP-based two-tier cellula network deployment of $20 \mathrm{~km} \times 20$

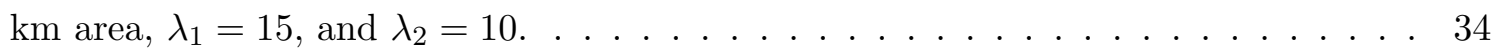

2.6 An illustration of a two-tier HetNet model of coverage area $(5000 \mathrm{~m} \times 5000 \mathrm{~m})$, $\lambda_{k}=20, \lambda_{u}=80, \mathrm{r}=500 \mathrm{~m}$, and $d_{\text {min }}=200 \mathrm{~m} \ldots \ldots \ldots \ldots$

2.7 Summary of dissertation outline. . . . . . . . . . . . . . . 39

3.1 Optimum length varying number of interfering cells $(\mathrm{L})$ for $U=10 \ldots \ldots$. . . . . 51

3.2 Optimum pilot length with no pilot contamination for various scaling factors for

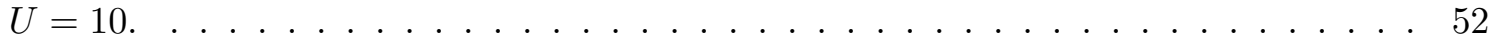

4.1 Interference power in ultra dense HetNets with ten Micro BSs . . . . . . . . . . . 54

4.2 Distances between Macro BS and Micro BSs . . . . . . . . . . . . . . . . . . 59

4.3 Distance between Micro BS and UE . . . . . . . . . . . . . . . 63

4.4 Distribution of Micro BSs around the Macro BS. . . . . . . . . . . . . 70

4.5 Flow chart for our proposed distance optimization algorithm. . . . . . . . . . . 71 
4.6 Micro-Micro interference comparison (coming from seven adjacent Micro BSs) for the first optimization problem. . . . . . . . . . . . . . . 74

4.7 Positions of three BSs from $[1] \ldots \ldots \ldots \ldots \ldots \ldots$

4.8 Position of three BSs after applying our suggested first optimization algorithm . . . 78

4.9 Average energy efficiency comparison before and after optimization . . . . . . . . 79

4.10 Comparison of our work with [1] for the second optimization problem. . . . . . . . 80

4.11 Comparison between interference power values for the second optimization problem . 81

5.1 An explanation of various interference levels in our model. . . . . . . . . . . . . . 85

5.2 An illustration of considered scenario: $B S_{1}$ gets interference from surrounding BSs $\quad 87$

5.3 Flow chart of our proposed MDIC algorithm _ . . . . . . . . . . . . . . 90

5.4 The total interference: Sum of (Macro-Macro), (Macro-Micro) and (Micro-Micro) interference before and after optimization . . . . . . . . . . . . 96

5.5 An illustration of how Macro cells became independent units and interference free. . 98

5.6 Interference power between Micro BSs associated with every Macro BS . . . . . . . . 99

5.7 Threshold combinations changing $\gamma_{1}$ related to Fig. 5.10 and Fig. 5.11. . . . . . . . . 99

5.8 Threshold combinations changing $\gamma_{2}$ and $\gamma_{4}$ related to Fig. 5.12. . . . . . . . . 100

5.9 Threshold combinations changing $\gamma_{3}$ related to Fig. 5.13. . . . . . . . . . . 100

5.10 Sum of interference in the whole network before and after optimization changing $\gamma_{1} .101$

5.11 Sum of interference in the whole network with various $\gamma_{1}$ values. . . . . . . . . . 102

5.12 Sum of interference in the whole network changing $\gamma_{2}$ and $\gamma_{4} \ldots \ldots$. . . . 103

5.13 Sum of interference in the whole network changing $\gamma_{3} \ldots \ldots \ldots 4$

5.14 Interference sum comparison for two threshold levels, ' 1 ' refers to $\gamma_{5}=0.005$ watts and '2' refers to $\gamma_{5}=0.008$ watts. . . . . . . . . . . . . . 105

6.1 Proposed optimization for user distribution . . . . . . . . . . . . . . 111

6.2 Explanation of the proposed user association algorithm selection criteria along iterations112

6.3 Selection criteria from all available combinations . . . . . . . . . . . . . . 113

6.4 Flow chart of the proposed user association algorithm. . . . . . . . . . . . . 114

6.5 User distribution changing as standard deviation converges . . . . . . . . . . 115

6.6 Transitional iterations as load in one BS changes . . . . . . . . . . . . . 116 
6.7 Comparison between network load of the proposed optimum standard deviation user association versus Max-SINR . . . . . . . . . . . . . 118

6.8 Comparison between the percentage of used bandwidth of the proposed standard deviation user association versus Max-SINR . . . . . . . . . . . . . . . 119

6.9 Comparison between network load standard deviation for various UE numbers . . . 120

6.10 Comparison between network load standard deviation for various Macro BS powers . 121

7.1 Selection criteria for UEs at every giving BS and for the accepting BS . . . . . . 125

7.2 Flow chart for the first iteration of our proposed RTLB user association algorithm. . 129

7.3 An illustration of handover from one cell to another. . . . . . . . . . . . . . 132

7.4 Initial data set for RTLB model for a scaled grid area of $4000 \times 4000 \mathrm{~m}$ and 500 total

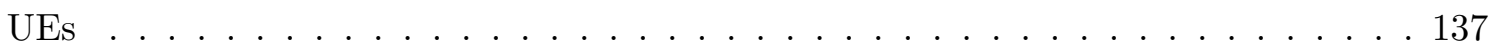

7.5 An illustration of algorithm performance along iterations as user calls are added or dropped . . . . . . . . . . . . . . . . . . . . . . . . . . . . . . .

7.6 An illustration of how our proposed algorithm supports variation in active users . . 138

7.7 An illustration of the elapsed time for RTLB versus LSTD . . . . . . . . . . . 139

7.8 BER Comparison for RTLB versus max-SINR . . . . . . . . . . . . . 140

7.9 Proposed Algorithm supports users' motion . . . . . . . . . . . . . . . . . 141

7.10 Comparison between network load of the proposed algorithm versus other algorithms

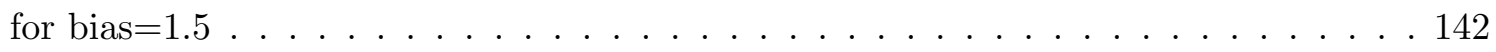

7.11 Average Load Per Tier BS Comparison . . . . . . . . . . . . . . 143

7.12 CDF of rate comparison for various association algorithms . . . . . . . . . 144 


\section{List of Tables}

1.1 Comparison between Homogeneous and Heterogeneous Networks. . . . . . . . . . 8

2.1 Comparison between spacial multiplexing, space-time coding, and spatial modulation. 18

2.2 Different detection techniques. . . . . . . . . . . . . . . . . 19

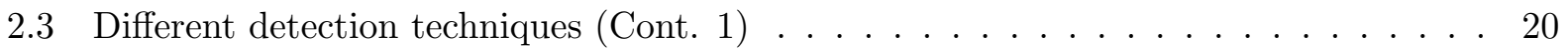

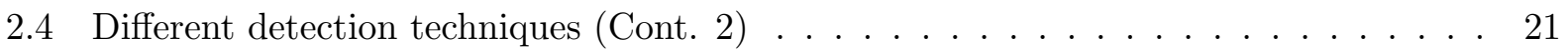

2.5 Different detection techniques $($ Cont. 3$) \ldots \ldots \ldots \ldots \ldots \ldots$

2.6 Various channel estimation techniques. . . . . . . . . . . . . 26

2.7 Various channel estimation techniques (Cont. 1) . . . . . . . . . . . . 27

2.8 Various channel estimation techniques $($ Cont. 2) $\ldots \ldots \ldots \ldots$

3.1 Comparison of related pilot length optimization work in literature. . . . . . . . . 44

4.1 Previous work done on PPP-based cellular networks . . . . . . . . . . . 55

4.2 Caption for $\mathrm{LOF} \ldots \ldots \ldots \ldots \ldots \ldots \ldots$

5.1 Parameters used for Simulation . . . . . . . . . . . . . . . . 95

6.1 Parameters used for LSTD user association algorithm . . . . . . . . . 117

7.1 Parameters used for RTLB user association algorithm . . . . . . . . . 136 


\section{List of Acronyms}

3D three dimensional

MIMO Multiple Input Multiple Output

SISO Single Input Single Output

MMSE Minimum Mean Squared Error

MSE Mean Squared Error

BS Base Station

UE User Equipment

5G Fifth Generation

SER Symbol Error Rate

BER Bit Error Rate

HetNets Heterogeneous Networks

CSI Channel State Information

LS Least-Squared

AOA Angle of Arrival

SNR Signal to Noise Ratio

SINR Signal to Interference and Noise Ratio

RSS Received Signal Strength 
MRC Maximum Ratio Combining

ZF Zero Forcing

OFDM Orthogonal Frequency Division Multiplexing

OFCDM Orthogonal Frequency and Code Division Multiplexing

TDMA Time Division Multiple Access

FDMA Frequency Division Multiple Access

CDMA Code Division Multiple Access

MCI Multi Code Interference Cancellation

ZF-SIC Zero Forcing Successive Interference Cancellation

LSTD Least Standard Deviation

RTLB Real Time Load Balancing

Std Standard Deviation

ID Identification Number

PPP Poisson Point Process

DIC Dummy Intersecting Circle

MDIC Modified Dummy Intersecting Circle

2D Two Dimensional

LTE Long Term Evolution

mmWave Millimeter Wave

MU Multiuser

WSN Wireless Sensor Networks

CSIT Channel State Information at the Transmitter 
TDD Time Division Duplexing

FDD Frequency Division Duplexing

DFT Discrete Fourier Transform

LR Lattice Reduction

MCMC Monte Carlo Markov Chain

PTP Point to Point

RF Radio Frequency

NMSE Normalized Mean Squared Error

SU-BF SISO or Single User Beamforming

FFR Fractional Frequency Reuse

QoS Quality of Service

ASE Area Spectral Efficiency

PN Pseudo Noise

PMF Probability Mass Function

OPLI Optimum Positions for Least Interference

RS Relay Stations

ICIC Inter Cell Interference Coordination

eICIC Enhanced Inter Cell Interference Coordination

ABS Almost Blank Subframes

STBC Space Time Block Coding

SDMA Space Division Multiple Access

SBS Selective Small Cell BS Deployment 
CRE Cell Range Expansion 


\section{List of Symbols}

$B_{n}$ Macro BS Antennas

$A_{n}$ Micro BS Antennas

$M$ Number of Macro BSs

$C$ Index of Micro BSs; $c=1,2,3, \ldots . C$

$U$ Index of Single Antenna Users; $u=1,2,3, \ldots . U$

$K$ Index of Tiers $; k=1,2,3, \ldots . K$

$\phi_{k}$ Poisson Process

$\lambda_{k}$ Poisson Process Density

$\rho_{k}$ BS Density Ratio Factor

$\tau$ Pilot Sequence Length

$p_{u}$ Pilot Power

$n_{j}$ Additive White Gaussian Noise

$P_{M}$ Macro BSs Transmitted Power

$P_{m}$ Micro BSs Transmitted Power

$P_{u}$ User Transmitted Power

$T$ OFDM Subcarrier Index; $t=1,2,3, \ldots . T$

$N_{b}$ Number of Transmitted Bits

xviii 
$S$ Total Available Data Streams Associated With Each UE

$N$ OFCDM Spreading Factor in Time Domain

F OFCDM Spreading Factor in Frequency Domain

$E_{b}$ Bit Energy

$E_{c}$ Chip Energy

$T_{c}$ Chip Duration

$Z$ OFCDM Subcarriers Per Group

$G_{d}$ OFCDM Subcarrier Groups with Index $d$

$\alpha$ Path Loss Exponent

$d_{\min }$ Min. Allowed Distance Between Micro BSs

$d_{c p}$ Min. Allowed Distance Between Macro and Micro BSs

$\beta_{j j}, \beta_{j l}$ Propagation Coefficients of Large-Scale Fading

$N_{t}$ Total Number of UEs in all Tiers

$N_{a}$ Number of Active UEs

$N_{m}$ Number of Active Mobile UEs

$N_{D}$ Number of Dropped UEs

$N_{d}$ Number of Added UEs

$W_{k}$ Total Bandwidth Assigned to Each BS

$L_{k}$ BS load

$\gamma_{k}$ SINR for the downlink of a given BS to its associated UE

$\zeta_{k}$ SINR Threshold

$P_{T_{k}}$ Tx power From a Tier BS 
$P_{c}\left(\gamma_{k}\right)$ Coverage Probability

$P_{s t_{k}}$ Static Power of BS in the $k^{t h}$ Tier 
CHAPTER 1

\section{Introduction}

Heterogeous networks (HetNets) are gaining the attraction of the communication society as they work in multi-layers cooperating together to fulfill the future dream of connecting the globe as one big network. Viewing the communication system as three dimensional (3D) with various tiers cooperating among each other is a new trend to present 5G HetNets. Base station (BS) in each tier operate with different power levels, access methods, and unique topologies. Instead of growing the communication system horizontally in one dimension, the future networks with dense nodes packed together will be grown in multiple dimensions and every dimension has its own unique characteristics. HetNets present a revolution in the future of communication systems and studying them with more depth will enable merging and cooperation between different networks throughout the globe. Everything in HetNets is variable; transmission power, access methods, cell coverage area and shape, antennas' sizes, and more.

\subsection{Homogeneous Single-tier Networks}

This represents a wireless network of spatially distributed Macro BSs. A certain user is served by the BS within its range of operation and undergoes handover to another BS with highest signal power when it changes its location. In homogeneous cellular networks, the system has a single network, with a number of BSs - either cooperating or non-cooperating to serve a number of simultaneous homogeneous users. Typically, in those systems, the BSs were identical in terms of average transmit 
power per unit area, access techniques, number of antennas, modulation and estimation schemes.

Macro BSs are high power nodes that are scattered uniformly along the geographical area to provide the following purposes:

- They are fundamental and core elements of any network to provide coverage and sufficient capacity to various parts.

- Every Macro BS covers up to $20 \mathrm{~km}$. So a less number of BSs is required to cover a certain area.

- In the past, the goal of any efficient system was to design high-performance, higher power, and energy-efficient BSs to reach distant and rural areas.

- Support a larger number of subcarriers.

- Interface with subcarriers using various access methods Time Division Multiple Access (TDMA)/Frequency Division Multiple Access (FDMA)/Code Division Multiple Access (CDMA).

- Used for coverage maximization.

- Used for interference reduction.

- As Macro BSs are very large, Massive number of antennas can be installed on it (Massive Multiple Input Multiple Output (MIMO)) which provides many benefits as:

1. A broad range of states of freedom, and greater selectivity in transmitting and receiving the data streams.

2. Improves capacity and reliability.

3. Channel estimation quality per antenna also improves with the number of BS antennas especially in the presence of high correlation among the antennas which is very typical [2].

4. Concentrates the released energy into small user centric zones, which dramatically increases throughput, energy efficiency [3], and latency.

5. Makes a proper use of beamforming techniques to reduce fading drops; this further boosts Signal to Noise Ratio (SNR), bit rate and reduces latency [4]. 
6. The channel becomes more predestined (due to channel hardening), and random detectors matrices are readily solved.

7. Interference cancellation is enhanced, where BSs can relatively easily avert transmission into undesired directions to alleviate harmful interference which, leads to low latency as well.

8. A higher number of BS antennas revokes the effects of uncorrelated noise and small-scale fading, and lowers the required transmitted energy per bit [5].

9. The more the antennas used, the finer the spatial focusing can be.

10. Aggressive spatial multiplexing in massive MIMO systems leads to an impressive improvement in the network capacity by minimizing Multiuser (MU) interference by steering the signal accurately in the right direction.

11. Furthermore, increasing the number of BS antennas above the number of active users leads to higher throughput [3].

12. The eigenvalue histogram of a single implementation converges to the average asymptotic eigenvalue distribution [6]. This leads to the possibility of employing simple low complexity detection techniques while preserving an excellent performance.

13. The more the BS antennas used, the more the data streams can be released to serve more terminals, reducing the radiated power, while boosting the data rate.

- It is used to connect large areas together and is used in areas where cables cannot be installed.

\subsubsection{Research Challenges in Homogeneous Networks}

However, Homogeneous networks technology suffered from some shortcomings like:

- Macro base stations provide poor quality of service at the cell edges.

- A large percentage of the cell area is uncovered properly.

- They are larger in size and provide a very large vacant deployment area.

- They deliver very high power levels, which are dangerous to be installed near civilian inhabited areas with large populations due to the risk of danger and diseases. 
- Not easily maintained.

- Their locations are fixed and once installed they are hard to be redeployed.

- Macro cells are affected by multipath signal loss.

- As communication is over the air and through large distances, it is subjected to scattering, attenuation, signal loss, physical obstructions, and climate conditions.

- It requires higher installation cost and the use of analog and hybrid approaches to cut down cost, which is primitive in the 21st century.

- Higher out-of-band radiation and high internal power consumption.

- The data must be accessible by every processing unit in order to compute inverse matrices. For a centralized system this will not be a problem, but for distributed systems all processors must have access to all of the data all of the time.

- It offers less data rate compare to wired networks such as fiber optics

\subsection{Heterogeneous Multi-tier Networks}

\subsubsection{Small cell Base Stations}

Small cells have been used recently and going small is thought of providing a better performance. Small cells mostly are used for indoor networks. Small cells provide flexibility in movement from one location to another and ease of installation. Also, they employ Less deployment, upgrading, and maintenance costs. Furthermore, they are more environmentally friendly as it carries less risks and pollution. They provide lower latency on the user end. For the performance advantage, we can list them as shown below:

- They provide an increased in Quality of Service (QoS) capabilities.

- Expand the capacity of wireless networks.

- Support higher capacity than Macro cells.

- Support high data rate. 
- Meet the demands for data, video, and applications.

- They provide an output power level of up to several watts.

- They are mounted on a lower height, so are less affected by interference.

However, small cell BSs have few advantages, which increases the need for a system that integrates both large and small cell BSs to benefit from advantages of both systems together and to limit the shortcomings. Small cell BSs support less number of subcarriers and have limited capabilities. Also, if they are close to each other, the interference level increases and managing the dense population becomes even harder. Besides, for outdoor applications backhaul options are limited.

\subsubsection{Heterogeneous Networks Benefits}

Due to the emerging small cell networks that provides many benefits, it became very vital to interact and integrate with the old Homogeneous Macro cell systems. The new born technology was termed as Heterogeneous networks, which is a network of two or more tiers connected together and every tier BSs has its own unique characteristics (delivers a certain power level, works with a certain access method, and serves a certain coverage area). In that new being, UEs have the capability to be associated to any tier BS and several interfaces are used to carry the information from one tier to another. The new trend in wireless communications to meet the growing demand is the introduction of low power nodes (Femto-cell, Pico-cells, Wi-Fi access points, distributed antennas, etc.) with heterogeneous users. In these HetNets, where there are multiple tiers working together at the same time, the capacity increases tremendously.

As our dissertation will be dealing with Heterogeneous networks, we will highlight some of the advantages of applying Heterogeneous networks as shown below:

- Interference level is reduced.

- Distributed approach works better and data exchange between the devices is not necessary.

- Improves coverage for large areas especially small cells.

- Supports user mobility in a better way.

- Easier to upgrade and maintain small cells. 
- Signals can reach very small areas with high level and coverage is maximized.

- Less cost for installing small cells.

- Acts as a solution to the problem of poor quality of service at the cell edges.

- Better outdoor-to-indoor coverage.

Heterogeneous networks have the following features:

- Different parts of the network are connected with different ways (wired, wireless, optical fiber, etc..).

- BSs operate with various transmission powers.

- Cells have different sizes and shapes, where the hexagonal cell shape presumption no longer exists.

- Users have the flexibility to connect to connect to any BS in any tier.

Figure 1.1 shows a PPP-based two-tier cellular network with a working area of $20 \mathrm{~km} \times 20 \mathrm{~km}$ area, consisting of Femto-cells (crosses) and Macrocells (dots). With the aid of stochastic geometry, the BS locations in each tier were modeled using a dependent or independent PPP to distribute the BSs randomly in different locations. The users associated with those BSs were modeled using a dependent PPP (using a parent-child relationship).

\subsubsection{Comparison of Homogeneous and Heterogeneous Networks}

HetNets can either be open access or closed access. In open access HetNets, the users can work under any BS in any tier, whereas in closed access networks, the user has limited access to certain tiers only, or has access to only the BSs in its own tier. The capacity and network lifwe time are higher in HetNets. Also, in HetNets the network has more capabilities, flexibility, and degrees of freedom. Table 1.1 highlights the differences between homogeneous and heterogeneous networks.

To emphasize the advantages of using HetNets over homogeneous networks, we plot and compare the performance of a two-tier HetNet and a homogeneous system as shown in Fig. 1.2. Fig. 1.2a shows a comparison of the NMSE versus SNR for the one-tier and two-tier system models. It is 


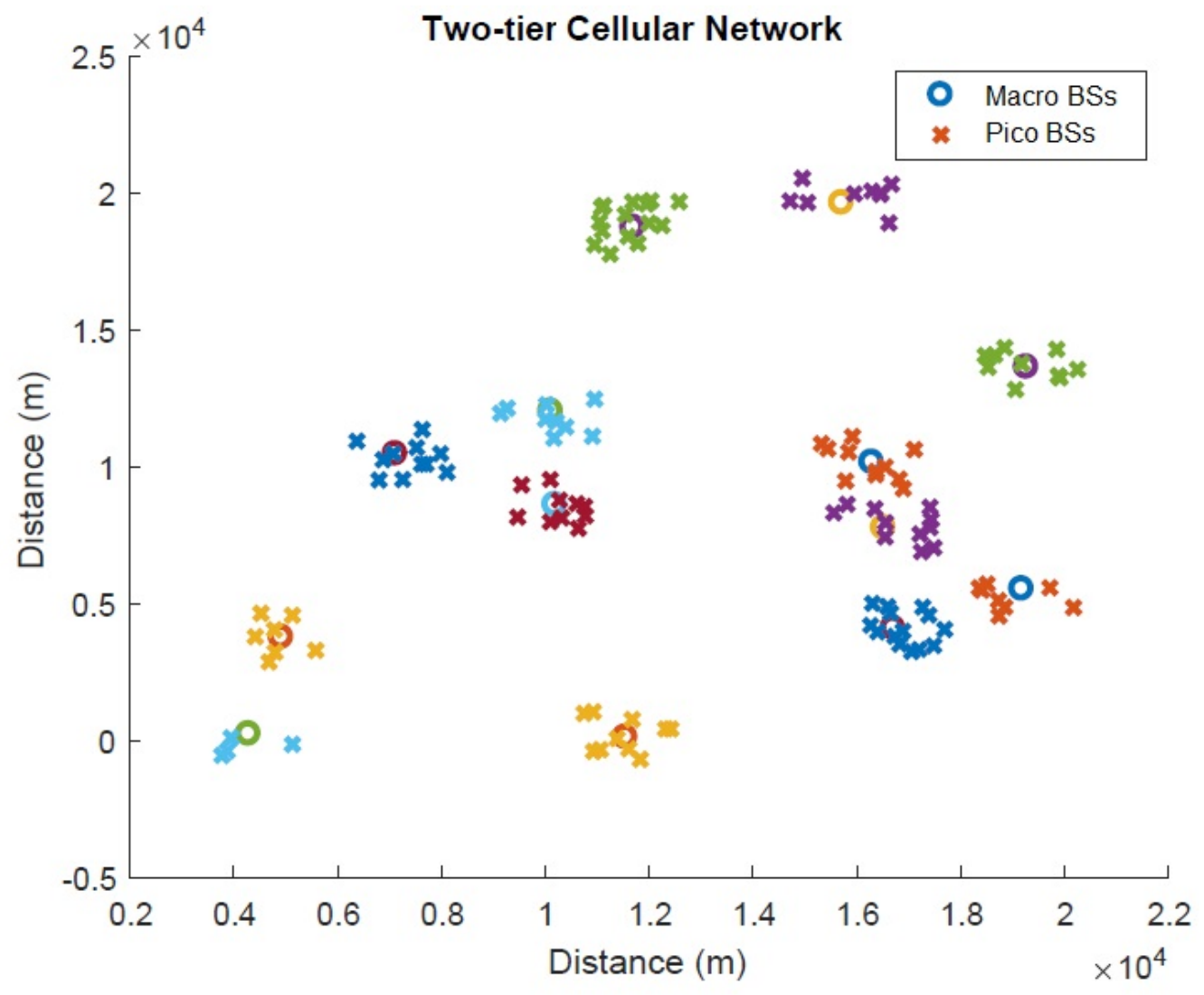

Figure 1.1: An illustration of a PPP-based two-tier cellular network deployment. A $20 \mathrm{~km} \times 20 \mathrm{~km}$ area, consisting of Femto-cells (crosses) and Macrocells (dots), is shown. 
Table 1.1: Comparison between Homogeneous and Heterogeneous Networks.

\begin{tabular}{cc}
\hline Heterogeneous Networks & Homogeneous Networks \\
\hline $\begin{array}{c}\text { Models the actual systems, which are composed of } \\
\text { multiple networks operating together at the same } \\
\text { time, and area }\end{array}$ & Non-realistic \\
\hline High sum-capacity in the order of thousands & Capacity increase, but not as HetNets \\
\hline Users can access any BS in any tier & Users access only the closest BS \\
\hline More interference is considered (inter-tier & Less affected by interference \\
\hline Flexible user choice and scheduling & User Selection is not flexible \\
\hline More flexibility and degrees of freedom & Less interference \\
\hline Optimal solutions to beamforming & the BS \\
\hline Networks with different capabilities & Limited \\
\hline More error rate & Less error rate \\
\hline BSs have different transmit powers, and multi-access \\
methods
\end{tabular}




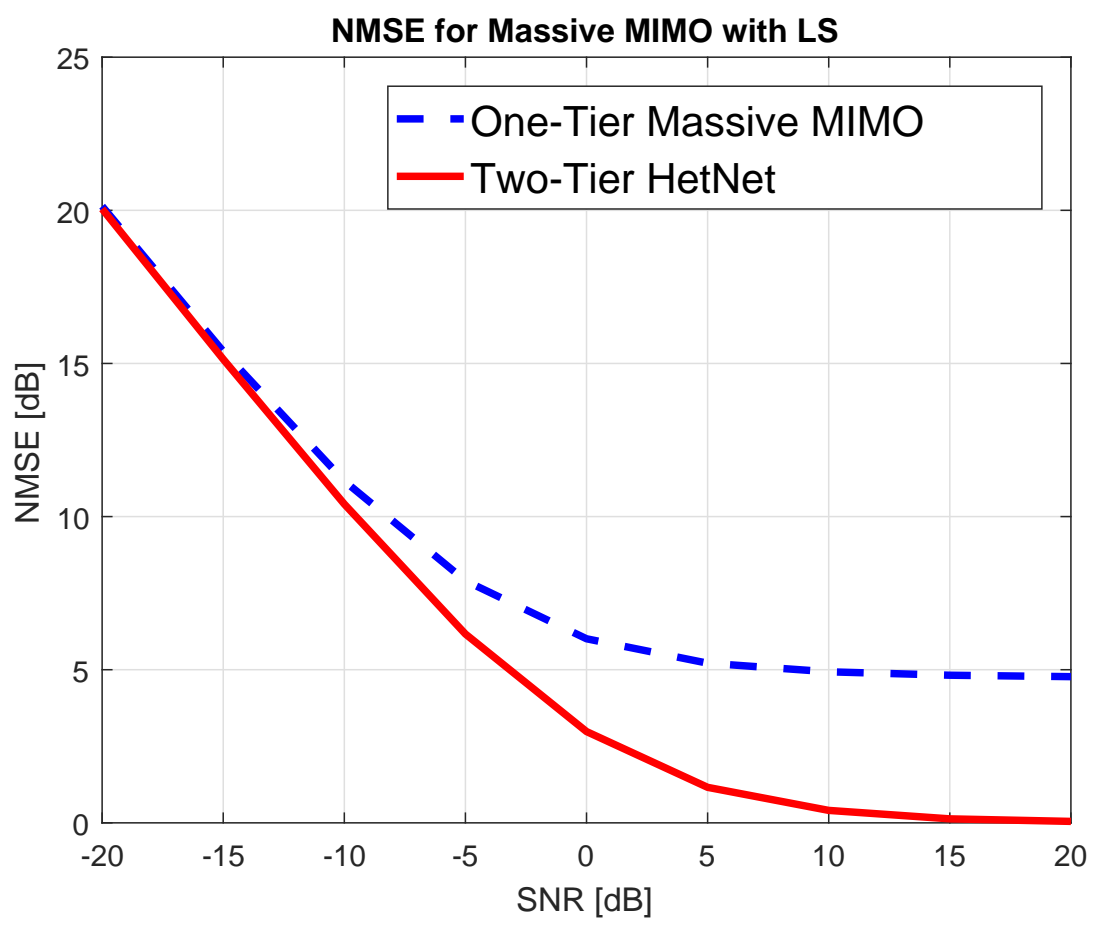

(a) NMSE for one-tier and two-tier systems.

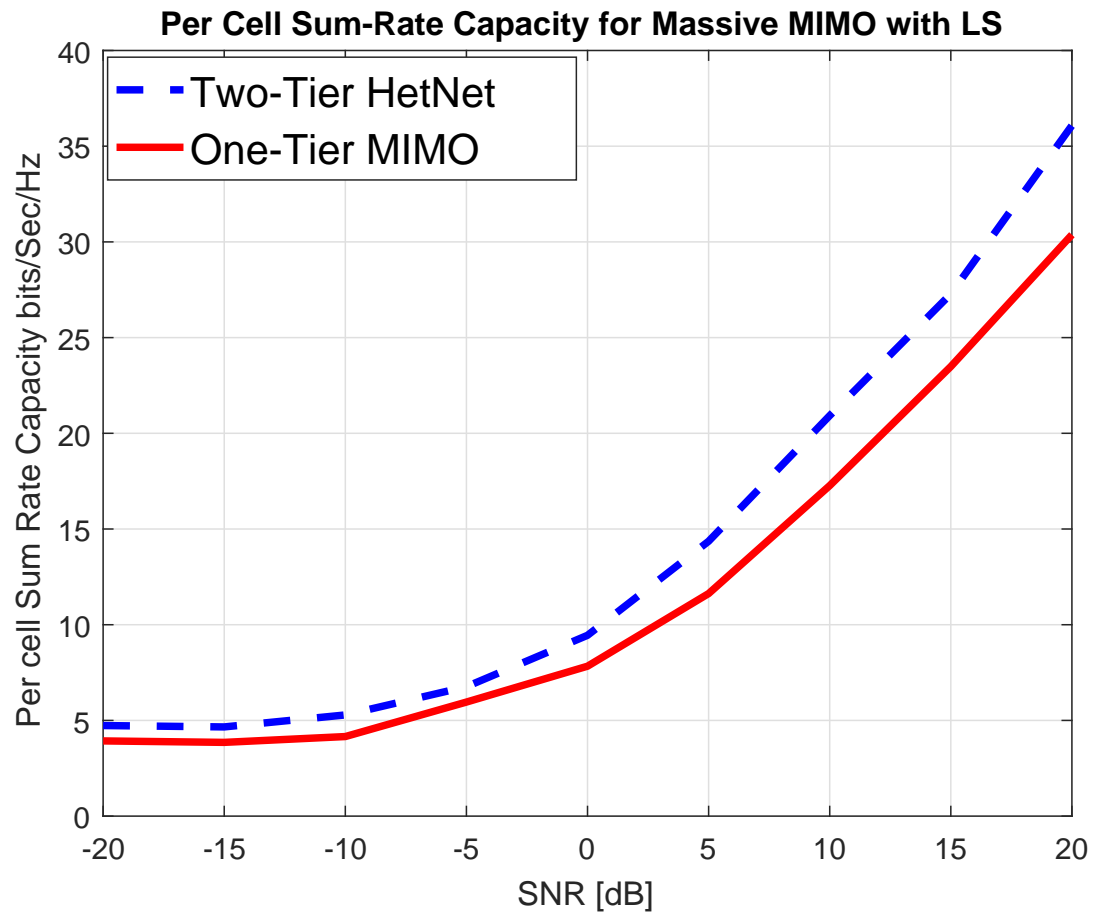

(b) Sum-Rate capacity for one-Tier and two-Tier systems.

Figure 1.2: NMSE and Sum-Rate capacity comparison 
clearly shown that the error is reduced in two-tier systems. Fig. 1.2b shows a comparison of the sum rate capacity (Bits/Sec/Hertz) versus SNR for the one-tier and two-tier systems. The capacity for the two-tier system is higher.

\subsubsection{Research Challenges in Heterogeneous Networks}

HetNets are arising on the surface due to the mandatory integration between the old single-tier networks and the newly introduced small cells. However, there are many research challenges that face the wide spread application of HetNets. Incase of applying Massive MIMO along with the HetNet technology, the problem of excess unnecessary antennas in the network arises. Small cells can take thefunction of the large number of MIMO antennas providing an adequate capacity and system performance. When integrating both systems together, those additional antennas might be of no use. Excess antennas increase intra and inter-tier interference in such ultra-dense networks. Also, it increases the delay spread, processing time, and load overhead. In addition, transmitted power to a user affects SINR level to the rest of the users; this effect should be compensated by careful choice of beamforming weights. Also, another serious problem that needs more attention is the requirement for proper synchronization between various parts and tiers. All access methods in all tiers need to be integrated into one access method. As small cells are very close to each other with the use of many antennas for transmission, combating high interference level in ultra dense small cells is becoming mandatory.

\subsection{Massive MIMO and Pilot Contamination}

\subsubsection{Massive MIMO}

Massive MIMO technology has got much attraction lately as it Promises truly broadband wireless networks [7]. Link reliability will also improve through spatial diversity and, it provides more degrees of freedom in the spatial domain, and improves the performance irrespective of the noisiness of the measurements. Besides, it provides a broad range of states of freedom, and greater selectivity in transmitting and receiving the data streams. Since all of the users can take part in the multiplexing gain, costly antenna array deployments are only necessary on the BS side, which saves on costs by sharing. This also leaves the user equipment less complex, often with a single antenna. 


\subsubsection{Pilot contamination}

When a signal propagates from the transmitter to the receiver, it experiences the effect of shadowing, scattering, fading, and path loss. Knowing the CSI in both forward and reverse links is crucial for accomplishing successful transmission under various channel conditions. Training sequences are often designed to optimize an equalizer at the receiver by providing CSI. In massive MIMO systems, the pilot sequence is used to estimate the CSI in both directions. For downlink transmission, the pilot sequences' sample period should be longer than the number of transmitting BS antennas. For uplink transmission, the pilot sequence sample period should be longer than the number of users, so that the BS would learn the uplink channel matrix.

Pilot contaminations results due to sending identical pilot sequences from users in adjacent cells and this would result in high channel estimation error. Also, covariance matrix estimation becomes more difficult.

Pilot contamination is a crucial problem in massive MIMO, which is caused by non-orthogonality of pilot sequences used in adjacent cells. Usually, reusing pilots in multiple cells is the main cause of the problem. In this case, the estimated channel vector in any cell is the summation of all the channel vectors of users from the neighboring cells (in addition to the original cell). As the number of interfering cells increase, the problem exponentially grows and eventually causes system malfunction.

Various solutions were introduced to solve this problem, which are:

- Channel Estimation Methods: These are based on some channel estimation algorithm to detect the CSI by picking up the strongest channel impulse responses, often done with less number of pilots than users.

- Time-Shifted Pilot Based Methods: These are based on insertion of shifted pilot locations in slots (or a shifted frame structure).

- Optimum Pilot Reuse Factor Methods: These are based on choosing a reuse factor greater than unity optimized in some sense. In addition, please note there are significant performance gaps that exist among different reuse patterns.

- Pilot Sequence Hopping Methods: These schemes switch users randomly to a new pilot 
between time slots, which provides randomization in the pilot contamination.

- Cooperative Methods: Here, each BS tries to find unique optimum pilots that are also suitable for other BSs.

- Cell Sectoring based Pilot Assignment: These schemes are based on sectioning the cells into a center and edge regions. Users in neighboring border areas partly reuse sounding sequences. This improves the quality of service by reducing the number of serviced users. However, by significantly reducing serviceable users, it degrades the system capacity.

- Angle of Arrival (AOA) based methods: Use the fact that non-overlapping user terminals reusing the pilots would have different AOA. However, this needs a way to detect AOA such as directional antennas.

There are other interconnected design issues that need to be properly understood and solved before widespread deployment of the massive MIMO technology. Several open research challenges are still facing the progress and development of this emerging technology which are:

\section{High deployment cost}

2. When more users are available; more pilot symbols are required to differentiate between users so training time goes up.

3. More advanced processing capabilities, precoding techniques, sophisticated channel estimation and sounding techniques, and acquisition and synchronization are required at the terminals. Also, the effect of hardware impairments becomes more clear and vigorous.

4. As detection becomes harder when the number of BS antennas increases, more advanced signal processing methods are required for better detection and are associated with introducing low complexity optimum and nonlinear detectors, and precoders to improve the performance and reduce the computational complexity.

5. For best beamforming, information of channels that is continuously changing should be known, so a lot of resources for downlink reference signal should be allocated which would cause waste of resources. 
6. More research is needed to introduce new adaptive beamforming techniques to achieve higher received symbol power and less interference.

7. Introducing efficient beamformers for Point to Point (PTP) networks to work under different constraints and with different types of channels would be beneficial for enabling PTP widespread application in massive MIMO systems.

8. Adding up multiple antenna output to a single beam will cause the width of the resulting beam to get narrower. Hence, the area covered by a beam would be very narrow and beamforming should be very quick, but this is challenging when the UE is fast moving.

9. Calibrating those huge number of antenna paths is challenging.

\subsection{Optimizing Base Stations Locations}

In this dissertation, we addressed BSs locations optimization and studied its effect on interference level, signal power, and coverage. BSs can be fixed or redeployable. Choosing the proper BSs locations has a direct impact on system performance. Changing BSs locations has an impact on the following:

- Interference level.

- The effect of deviations in the site locations is closely related to the average distance between BSs.

- Received pilot power is affected.

- Received data power.

- For a centralized approach, if locations of BSs are very far from the processing BS, performance is degraded as SINR level is affected.

- In order to make it possible for the customer on the move to continuously make or receive a call, the cells must necessarily overlap. The size of overlap area will have a direct effect on handover results.

- Coverage overlapping area. 
- Changing locations affects handover areas.

- The further BSs will be away from users, the poorer the signal quality and less battery life.

\subsection{User Association}

User association is about taking the proper decision on which user is more suitable for a certain BS. Sometimes, users are selected based on the highest signal power, the best signal quality, BS load, or overall network load. User association in HetNets is challenging due to their nature. Many algorithms were invented recently to address this challenge, however, there is no standard algorithm that researchers agreed upon that would be the best for HetNets. Sometimes, users were selected so as to optimize a certain network parameter like: energy efficiency, spectrum efficiency, QoS, fairness, and coverage probability [8]. User mobility has to be considered when choosing a certain association algorithm. An association algorithm that does not consider users' mobility will result in more handover frequencies than the conventional homogeneous networks, which in turn lead to more dropped calls and poor service quality.

User association is decided based on three approaches:

1. Centralized: The network contains a central unit that collects all information (data traffic, BSs load, signal power,....etc). Based on the received information, the central BSs decides which specific users will be associated to which BSs. This approach is the fastest, but it carries complicated signaling overhead.

2. Distributed: It is suitable for ultra dense large networks. There is no centre BS that takes over the processing overhead. Every BS and user in the network is responsible for its own decision by interacting with its neighbors. This approach tends to be slower, with less signalling overhead, but decisions do not consider the big picture of overall network performance and might be incorrect.

3. Hybrid: A centralized approach is used for one or more parameters, while a distributed approach is used for the rest of the parameters. 


\subsection{Chapter Summary}

Cellular systems can be Homogeneous or Heterogeneous. Homogeneous networks have been around for years and they have many advantages. The introduction of small cells improved QoS, and increased data rate. The integration between the old Homogeneous networks and the new small cells was a must to meet the rapidly growing data traffic. HetNet systems are an innovative technology that helps in the achievement of higher system throughput and reliable transmission for 5G and beyond wireless networks. We discussed the advantages and some challenges of using Homogeneous and Heterogeneous networks. Also, we studied BSs deployment and user association to BSs. 
CHAPTER 2

\section{System Model and its Components}

\subsection{Chapter Overview}

In this chapter, we describe the system model, and explain all its different components like the Poisson point process, multi-tier systems, Orthogonal Frequency and Code Division Multiplexing (OFCDM) systems, etc,.... Also, we explain in detail the reason for choosing every component and highlight its superiority over other conventional commonly used system components. In our system model, we considered a multi-tier HetNet system, where BSs in every tier are deployed according to a Poisson point process. Also we considered uplink pilot transmission and obtained an expression for optimum pilot sequence length, we considered uplink and downlink operations, we considered TDD for channel estimation, and we used OFCDM for the link between BSs and UEs. In this chapter, we explain in detail all the aforementioned terms and explain in detail the advantages of using them.

\subsection{Uplink and Downlink Operations}

Figure 2.1 shows the uplink and the downlink of single cell system, where the BS is composed of a few hundred service antennas serving a few hundred users each, usually with only one antenna. 


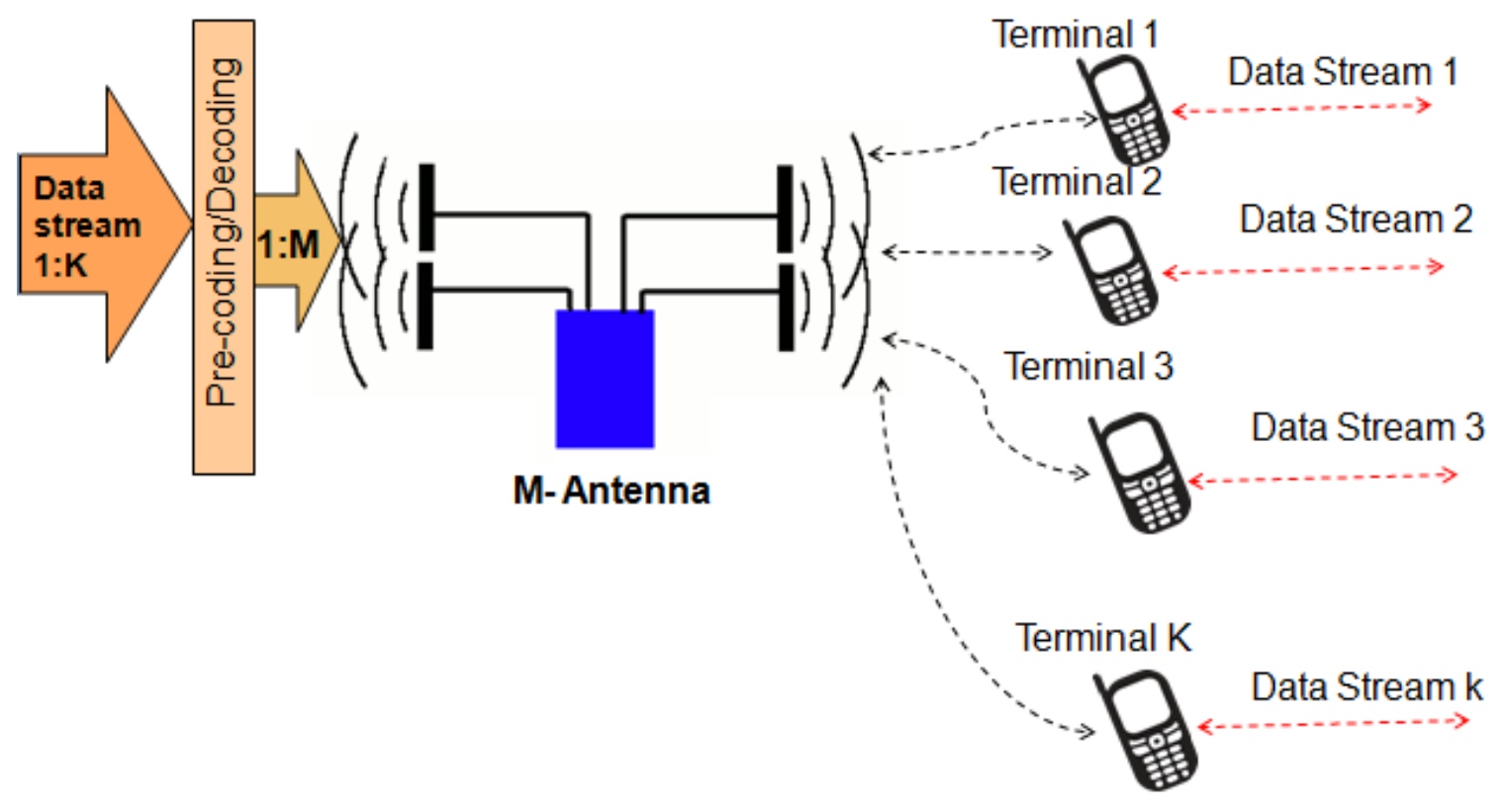

Figure 2.1: Uplink and downlink operation.

\subsubsection{Uplink Operation}

The communication going from the user side to the BS, or from the Micro to the Macro BS is called uplink. In chapter 3, we consider uplink operation, as the training phase is usually on the uplink side. Also, we apply uplink in chapter 5 for the communication from the dummy edge Micro BS to the Macro BS in the adjacent cell.

We will explain the uplink operation in more detail. Also, we highlight the different steps of this operation as follows [9]:

1. Encoding: Is employed to prepare data for transmission. Encoding is all about converting data into symbols appropriate for transmission over multiple transmit antennas. Space multiplexing and space-time coding are the commonly used encoding techniques, as they do not require knowledge of the CSI at the transmitter. Table 2.1 compares Spatial Multiplexing, Space-Time Coding, and Spatial Modulation. Encoding using known CSI at the transmitter is known as precoding [6].

2. Training: Pilot sequences and uplink data sequences are transmitted at the same time and 
Table 2.1: Comparison between spacial multiplexing, space-time coding, and spatial modulation.

\begin{tabular}{|c|c|c|}
\hline Spacial Multiplexing & Space-Time Coding & Spatial Modulation \\
\hline Achieves high rates. & $\begin{array}{c}\text { Achieves increased reliability through } \\
\text { transmit diversity. }\end{array}$ & $\begin{array}{l}\text { Allows fewer transmit Radio } \\
\text { frequency chains. }\end{array}$ \\
\hline $\begin{array}{l}\text { Information is carried on the } \\
\text { modulation symbols. }\end{array}$ & $\begin{array}{l}\text { Information is carried on the } \\
\text { modulation symbols. }\end{array}$ & $\begin{array}{l}\text { Information is carried on the } \\
\text { modulation symbols in addition to } \\
\text { the indices of the antennas on which } \\
\text { transmission takes place. }\end{array}$ \\
\hline $\begin{array}{l}\text { Simplest (only the receiver needs to } \\
\text { detect transmitted symbols). }\end{array}$ & Sophisticated. & $\begin{array}{l}\text { Simple, but requires additional } \\
\text { memory to construct encoding table } \\
\text { at the transmitter. }\end{array}$ \\
\hline Example: V-BLAST. & Space-Time Trellis. & Space Time Block Coding (STBC) \\
\hline
\end{tabular}

over the same frequencies from each user to the BS.

3. Estimation: The BS receives the sum of data streams from all the users, and estimates the channel. Receive combining is used in the uplink for differentiation between signals sent from different terminals.

4. Decoding and detection: They produce individual data streams by utilizing the estimated CSI. Received signals from different terminals are combined in the uplink using appropriate decoding. Signal detection implies accurate estimation of the transmit vector knowing the received vector and (sometimes) the channel. Detection of encoded signals is very demanding and probably the most important task, since the received signal is subjected to noise, fading, shadowing as well as spatial interference. Advanced signal processing methods are required for accurate detection. Since the elements of the transmitted vector belong to a predefined discrete alphabet, detection is harder when the alphabet is bigger. Some detection algorithms produce soft values of the estimate of the transmitted symbols while the others produce hard values $[6]$.

The estimated soft values are fed into the channel decoders in coded systems. Hard outputs like search-based algorithms test a set of discrete valued vectors and then choose the best one among them as the output. In general, the soft fed values give a better performance compared to hard inputs. The general trend has been to consider optimization algorithms and artificial intelligence to achieve superior detection performance. Detection can be done 
Table 2.2: Different detection techniques.

\begin{tabular}{|c|c|c|c|c|c|}
\hline Ref. & $\begin{array}{l}\text { Detection } \\
\text { strategy }\end{array}$ & System Type & $\begin{array}{c}\text { User } \\
\text { Antennas }\end{array}$ & Channel Type & $\begin{array}{c}\text { Performance } \\
\text { Metric }\end{array}$ \\
\hline$[10], 2008$ & Factor Graph & MU & Multiple & Dual-polarized & Sum-Rate \\
\hline$[11], 2010$ & Factor Graph & Single User & Multiple & Clustered Scattering & Mean Squared Error \\
\hline$[12], 2011$ & Factor Graph & Single User & Multiple & Ray-Tracing & Throughput \\
\hline [13], 2012 & Factor Graph & MU & Single & Ray-Tracing & Spatial Correlation \\
\hline$[14], 2012$ & Factor Graph & Single User & Multiple & $\begin{array}{c}\text { Frequency Selective } \\
\text { Fading Channel }\end{array}$ & Geometric Mean \\
\hline [15], 2013 & Factor Graph & Single User & Multiple & Block-Fading & $\begin{array}{c}\text { Average Probability of } \\
\text { Detection Error }\end{array}$ \\
\hline$[16], 2014$ & Factor Graph & $\mathrm{MU}$ & Multiple & Gaussian & Bit Error Rate (BER) \\
\hline$[17], 2003$ & $\begin{array}{l}\text { Interference } \\
\text { Cancellation }\end{array}$ & MU & Multiple & Flat Fading & BER \\
\hline$[18], 2007$ & $\begin{array}{l}\text { Interference } \\
\text { Cancellation }\end{array}$ & $\mathrm{MU}$ & Multiple & Complex Gaussian & BER \\
\hline$[19], 2008$ & $\begin{array}{l}\text { Interference } \\
\text { Cancellation }\end{array}$ & MU & Multiple & Gaussian & BER \\
\hline [20], 2009 & $\begin{array}{l}\text { Interference } \\
\text { Cancellation }\end{array}$ & Single User & Multiple & NA & $\begin{array}{c}\text { Block Error Rate and } \\
\text { Throughput }\end{array}$ \\
\hline$[21], 2009$ & $\begin{array}{l}\text { Interference } \\
\text { Cancellation }\end{array}$ & Single User & Multiple & NA & Bit Error Rate \\
\hline$[22], 2011$ & $\begin{array}{l}\text { Interference } \\
\text { Cancellation }\end{array}$ & MU & Multiple & Fading & Packet Error Rate \\
\hline$[23], 2011$ & $\begin{array}{l}\text { Interference } \\
\text { Cancellation }\end{array}$ & $\mathrm{MU}$ & Single & Flat Fading & BER \\
\hline$[24], 2012$ & $\begin{array}{l}\text { Interference } \\
\text { Cancellation }\end{array}$ & Single-user & Multiple & Complex Gaussian & $\begin{array}{c}\text { Achievable rate and } \\
\text { BER }\end{array}$ \\
\hline$[25], 2012$ & $\begin{array}{c}\text { Interference } \\
\text { Cancellation MUs }\end{array}$ & Multiple & $\begin{array}{l}\text { Complex } \\
\text { Gaussian }\end{array}$ & BER & \\
\hline$[26], 2012$ & $\begin{array}{l}\text { Interference } \\
\text { Cancellation }\end{array}$ & $\begin{array}{c}\text { Single and } \\
\text { Multiple Users }\end{array}$ & Multiple & $\begin{array}{c}\text { Multipath } \\
\text { Discrete-Time Block } \\
\text { Fading }\end{array}$ & BER \\
\hline$[27], 2012$ & $\begin{array}{l}\text { Interference } \\
\text { Cancellation }\end{array}$ & Single User & Multiple & Block Fading & BER \\
\hline$[28], 2014$ & $\begin{array}{l}\text { Interference } \\
\text { Cancellation }\end{array}$ & Single User & Multiple & NA & BER \\
\hline$[29], 2014$ & $\begin{array}{l}\text { Interference } \\
\text { Cancellation }\end{array}$ & MU & Multiple & NA & BER \\
\hline [30], 2014 & $\begin{array}{l}\text { Interference } \\
\text { Cancellation }\end{array}$ & $\mathrm{MU}$ & Multiple & Complex Gaussian & BER \\
\hline
\end{tabular}


Table 2.3: Different detection techniques (Cont. 1)

\begin{tabular}{|c|c|c|c|c|c|}
\hline Ref. & $\begin{array}{l}\text { Detection } \\
\text { strategy }\end{array}$ & System Type & $\begin{array}{c}\text { User } \\
\text { Antennas }\end{array}$ & Channel Type & $\begin{array}{c}\text { Performance } \\
\text { Metric }\end{array}$ \\
\hline$[31], 2016$ & $\begin{array}{l}\text { Interference } \\
\text { Cancellation }\end{array}$ & $\mathrm{MU}$ & Single & Flat Fading & $\begin{array}{c}\text { BER, and Average } \\
\text { Number of } \\
\text { Computations }\end{array}$ \\
\hline$[31], 2017$ & $\begin{array}{l}\text { Interference } \\
\text { Cancellation }\end{array}$ & $\mathrm{MU}$ & Single & Flat Fading & BER \\
\hline$[32], 2007$ & Linear & $\mathrm{MU}$ & Multiple & Rayleigh Block Fading & BER \\
\hline [33], 2009 & Linear & Single User & Multiple & $\begin{array}{c}\text { Rich-Scattering } \\
\text { Flat-Fading }\end{array}$ & BER \\
\hline$[34], 2011$ & Linear & $\mathrm{MU}$ & Multiple & Frequency-Flat Fading & $\begin{array}{l}\text { Empirical Cumulative } \\
\text { Distribution and BER }\end{array}$ \\
\hline$[35], 2011$ & Linear & Single User & Multiple & Block Fading & $\begin{array}{c}\text { Bit and Packet Error } \\
\text { Rate }\end{array}$ \\
\hline [36], 2012 & Linear & Single User & Single & Two-Way Relay & BER \\
\hline [37], 2013 & Linear & Multiple Users & Multiple & NA & Block Error Rate \\
\hline$[38], 2013$ & Linear & Single User & Multiple & Flat Fading & BER \\
\hline$[39], 2013$ & Linear & Single User & Multiple & $\begin{array}{l}\text { Time-Varying Flat } \\
\text { Fading }\end{array}$ & BER \\
\hline$[40], 2013$ & Linear & Single User & Multiple & NA & BER \\
\hline$[41], 2016$ & Linear & MU & Single & Block Flat Fading & Sum Rate \\
\hline$[42], 2008$ & Local Search & MU & Single & Rayleigh flat & BER \\
\hline$[43], 2015$ & Local Search & Single User & Multiple & NA & BER \\
\hline$[44], 2016$ & Local Search & $\mathrm{MU}$ & Single & Flat Fading & $\begin{array}{l}\text { Bit Error Rate, and } \\
\text { Average Number of } \\
\text { Arithmetic Operations }\end{array}$ \\
\hline$[45], 2016$ & Local Search & $\mathrm{MU}$ & Single & $\begin{array}{l}\text { Quasi-Static Flat } \\
\text { Fading }\end{array}$ & $\begin{array}{c}\text { Bit Error Rate, and } \\
\text { Normalized Spectral } \\
\text { Efficiency }\end{array}$ \\
\hline$[46], 2007$ & $\begin{array}{l}\text { Lattice Reduction } \\
\text { (LR)-Aided }\end{array}$ & MU & Multiple & NA & $\begin{array}{l}\text { BER, Packet error } \\
\text { rate and Throughput }\end{array}$ \\
\hline$[47], 2008$ & LR-Aided & $\mathrm{MU}$ & Multiple & $\begin{array}{l}\text { Flat-Fading } \\
\text { Quasi-Static }\end{array}$ & BER \\
\hline$[48], 2010$ & LR-Aided & Multiple Users & Multiple & Rayleigh faded & BER \\
\hline$[49], 2010$ & LR-Aided & Single User & Multiple & Flat-Fading & BER \\
\hline$[50], 2011$ & LR-Aided & Single User & Multiple & Flat-Fading & BER \\
\hline$[34], 2011$ & LR-Aided & Single User & Multiple & Frequency-Flat Fading & $\begin{array}{c}\text { Empirical Cumulative } \\
\text { Distribution Function } \\
\text { and BER }\end{array}$ \\
\hline
\end{tabular}


Table 2.4: Different detection techniques (Cont. 2)

\begin{tabular}{|c|c|c|c|c|c|}
\hline Ref. & $\begin{array}{l}\text { Detection } \\
\text { Strategy }\end{array}$ & System Type & $\begin{array}{c}\text { User } \\
\text { Antennas }\end{array}$ & Channel Type & $\begin{array}{l}\text { Performance } \\
\text { Metric }\end{array}$ \\
\hline$[51], 2012$ & LR-Aided & Single User & Multiple & $\begin{array}{l}\text { Quasi-Stationary } \\
\text { (Block Fading) }\end{array}$ & $\begin{array}{c}\text { Average Orthogonality } \\
\text { Defect and BER }\end{array}$ \\
\hline$[52], 2012$ & LR-Aided & Single User & Multiple & Rayleigh Flat-Fading & BER \\
\hline [53], 2012 & LR-Aided & Single User & Multiple & NA & BER \\
\hline [38], 2013 & LR-Aided & Single User & Multiple & Flat Fading & BER \\
\hline$[54], 2014$ & LR-Aided & Single User & Multiple & Flat Fading & BER \\
\hline$[54], 2014$ & LR-Aided & Three-User & Multiple & Frequency Flat Fading & BER \\
\hline [55], 2014 & LR-Aided & Single User & Multiple & Rayleigh Flat-Fading & $\begin{array}{c}\text { Channel Correlation } \\
\text { Effect and BER }\end{array}$ \\
\hline$[56], 2016$ & LR-Aided & Multiple Users & Multiple & Rayleigh Flat-Fading & $\begin{array}{c}\text { BER, Sum Rate, and } \\
\text { Plog Cond }\end{array}$ \\
\hline [57], 2016 & LR-Aided & Multiple Users & Multiple & $\begin{array}{l}\text { Quasi-Static Block } \\
\text { Fading }\end{array}$ & BER \\
\hline [58], 2017 & LR-Aided & MU & Single & Rayleigh Flat-Fading & $\begin{array}{c}\text { BER, and Average } \\
\text { Flops }\end{array}$ \\
\hline$[59], 2005$ & $\begin{array}{c}\text { Monte Carlo } \\
\text { Markov } \\
\text { Chain (MCMC) }\end{array}$ & Single User & Multiple & Flat Fading & BER \\
\hline$[60], 2007$ & MCMC & Single User & Multiple & Flat Fading & BER \\
\hline [61], 2008 & MCMC & Single User & Single & Block Fading & $\mathrm{BER}$ \\
\hline$[21], 2009$ & MCMC & Single User & Multiple & NA & BER \\
\hline$[62], 2011$ & MCMC & Single User & Multiple & NA & BER \\
\hline [63], 2011 & $\mathrm{MCMC}$ & Single User & Multiple & NA & BER \\
\hline [64], 2012 & $\mathrm{MCMC}$ & Multiple Users & Multiple & $\begin{array}{c}\text { Frequency } \\
\text { Non-Selective Fading }\end{array}$ & BER \\
\hline [65], 2015 & MCMC & Multiple Users & Single & NA & BER \\
\hline$[66], 2016$ & MCMC & Multiple Users & Single & NA & BER \\
\hline$[67], 2016$ & MCMC & Multiple Users & Single & NA & BER \\
\hline [68], 2006 & $\begin{array}{l}\text { Optimum } \\
\text { Detection }\end{array}$ & Single User & Multiple & $\begin{array}{l}\text { Uncorrelated Rayleigh } \\
\text { Fading }\end{array}$ & BER \\
\hline [69], 2007 & $\begin{array}{l}\text { Optimum } \\
\text { Detection }\end{array}$ & Single User & Multiple & Quasi-Static & Frame Error Rate \\
\hline [70], 2007 & $\begin{array}{l}\text { Optimum } \\
\text { Detection }\end{array}$ & Single User & Multiple & Flat Rayleigh Fading & BER \\
\hline
\end{tabular}


Table 2.5: Different detection techniques (Cont. 3)

\begin{tabular}{|c|c|c|c|c|c|}
\hline Ref. & $\begin{array}{l}\text { Detection } \\
\text { Strategy }\end{array}$ & System Type & $\begin{array}{c}\text { User } \\
\text { Antennas }\end{array}$ & Channel Type & $\begin{array}{c}\text { Performance } \\
\text { Metric }\end{array}$ \\
\hline$[71], 2009$ & $\begin{array}{l}\text { Optimum } \\
\text { Detection }\end{array}$ & $\mathrm{MU}$ & Multiple & NA & BER \\
\hline$[72], 2009$ & $\begin{array}{l}\text { Optimum } \\
\text { Detection }\end{array}$ & Single User & Multiple & Block fading & Frame Error Rate \\
\hline [73], 2015 & $\begin{array}{l}\text { Optimum } \\
\text { Detection }\end{array}$ & $\mathrm{MU}$ & Single & Rayleigh Fading & BER, and Flops \\
\hline [74], 2016 & $\begin{array}{l}\text { Optimum } \\
\text { Detection }\end{array}$ & $\mathrm{MU}$ & Single & Rayleigh Fading & $\begin{array}{c}\text { BER, Frame Error } \\
\text { Rate, and Normalized } \\
\text { Info Rates }\end{array}$ \\
\hline$[75], 2006$ & $\begin{array}{c}\text { Probabilistic Data } \\
\text { Association }\end{array}$ & $\mathrm{MU}$ & Multiple & $\begin{array}{c}\text { Quasi-Static Rayleigh } \\
\text { Fading }\end{array}$ & BER \\
\hline$[76], 2008$ & $\begin{array}{c}\text { Probabilistic Data } \\
\text { Association }\end{array}$ & Single User & Multiple & NA & BER \\
\hline [77], 2009 & $\begin{array}{c}\text { Probabilistic Data } \\
\text { Association }\end{array}$ & Single User & Multiple & NA & BER \\
\hline [78], 2011 & $\begin{array}{c}\text { Probabilistic Data } \\
\text { Association }\end{array}$ & Single User & Multiple & NA & $\begin{array}{c}\text { Bit and Symbol Error } \\
\text { Rate }\end{array}$ \\
\hline [78], 2011 & $\begin{array}{c}\text { Probabilistic Data } \\
\text { Association }\end{array}$ & Single User & Multiple & NA & BER \\
\hline [79], 2013 & $\begin{array}{c}\text { Probabilistic Data } \\
\text { Association }\end{array}$ & $\mathrm{MU}$ & Multiple & Nakagami-m Fading & BER \\
\hline [80], 2013 & $\begin{array}{c}\text { Probabilistic Data } \\
\text { Association }\end{array}$ & $\mathrm{MU}$ & Multiple & Nakagami-m Fading & BER \\
\hline [81], 2013 & $\begin{array}{c}\text { Probabilistic Data } \\
\text { Association }\end{array}$ & Single User & Multiple & Nakagami-m Fading & BER \\
\hline$[82], 2017$ & $\begin{array}{c}\text { Probabilistic Data } \\
\text { Association }\end{array}$ & $\mathrm{MU}$ & Single & Flat Rayleigh Fading & BER \\
\hline [83], 2008 & $\begin{array}{l}\text { Soft-Input } \\
\text { Soft-Output }\end{array}$ & $\mathrm{MU}$ & Single & Flat Rayleigh Fading & BER \\
\hline [84], 2009 & $\begin{array}{l}\text { Soft-Input } \\
\text { Soft-Output }\end{array}$ & MU & Single & $\begin{array}{c}\text { Rayleigh Multi-Path } \\
\text { Fading }\end{array}$ & $\begin{array}{c}\text { Average Complexity, } \\
\text { and Rate }\end{array}$ \\
\hline [85], 2014 & $\begin{array}{l}\text { Soft-Input } \\
\text { Soft-Output }\end{array}$ & $\mathrm{MU}$ & Single & Rayleigh Fading & Frame Error Rate \\
\hline
\end{tabular}


using linear or nonlinear algorithms. Linear detection generally generates soft estimates of the transmitted vectors [6]. Linear detection has less complexity but yields lower performance and limited spectral efficiency. The performance of linear detectors deteriorates rapidly as the number of transmitting antennas increases. LR based linear detection has better performance than ordinary linear detection. However, instead of applying the linear transformation to the received signal model, they apply it to an equivalent system model obtained using LR-techniques. The new channel matrix is more orthogonal than the old one. Slicing is done on the data vector instead of the transmitted vector. Interference cancelation detectors are nonlinear, which perform estimation and removal of interference in multiple stages. There are optimal detectors such as Maximum Likelihood Detectors. Local search detectors also seek for optimal solution. However, these methods have an issue of huge problem size, as well as the lack of knowledge of the problem structure. The good thing about local search is its neighborhood function that guides the search to a right solution. Another popular technique is the polynomial time approximation algorithm, but it gives an inferior solution. Tabu search is a mathematical optimization method that is used to solve combinatorial optimization problems. It is effective when the problem size gets very large. It has the ability of quickly find near-optimal solutions [6]. Low-complexity MIMO uses detection based on probabilistic data association. Remote sensing applications have been using this detection method for target tracking for some time. Since signals coming from the targets are weak, the detection threshold is lower. However, this leads to detection of additional unwanted signals and noise. Data association means to specify which measurements are the most suitable to be used in tracking filters. This technique's principal aim is to track targets where there is uncertainty in their data association [6]. Tables 2.2, 2.3, 2.4, and 2.5 show the different detection techniques.

\subsubsection{Downlink Operation}

The communication in the opposite direction, from the BS to the UE or from the Macro to the Micro BS is called downlink. We consider downlink operation in chapters 4 and 5. So, we will explain the different steps of the downlink process in more detail as follows:

1. Beamforming: Data streams are transmitted from the BSs to only the intended users by means of beamforming, where the different data streams may occupy the same frequencies at 
the same time (space division multiplexing).

2. Precoding: The previous operation is carried out knowing the frequency response of the propagation channels (or CSI) between each of its elements and each user and precoding the signals accordingly. Unlike the conventional MIMO, massive MIMO uses linear precoders, such as Maximum Ratio Combining (MRC), matched filtering, conjugate beamforming, Minimum Mean Squared Error (MMSE) receive combining, and ZF [2]. Linear precoding techniques at the downlink aim to focus each signal at its desired terminal and mitigate interference towards other terminals [3]. Low-complexity precoding methods are mandatory and critical to minimize the computational complexity of the precoder [86].

\subsection{Channel Estimation Methods: (TDD or FDD?)}

In our system model proposed in this dissertation, we considered Time Division Duplexing (TDD) for channel estimation. A non-stationary wireless channel needs to be re-estimated after every coherence time lap. Massive MIMO systems were originally envisioned for TDD operation, in which the channel is periodically estimated in one direction and compensation can be applied in both directions assuming reciprocity.

\subsubsection{Why did we use TDD?}

TDD systems have the following features:

1. The time required to acquire CSI does not depend on the number of BSs or users.

2. Only the BS needs to know the information about the channels to process antennas coherently.

In TDD systems, multi-user precoding in the downlink and detection in the uplink require CSI knowledge at the BS. The resource, time or frequency needed for channel estimation is proportional to the number of the transmit antennas.

\subsubsection{Why did not we use FDD?}

In Frequency Division Duplexing (FDD), uplink and downlink use different frequency bands (different CSI in both links). The uplink channel estimation at the BS is done by letting all users send different 
pilot sequences. To get the CSI for the downlink channel, the BS transmits pilot symbols to all users. The users respond by the estimated CSI for the downlink channels [2].

CSI can be estimated at the receiver side only, or at both at the transmitter and the receiver. Estimation at both sides has some advantages. The CSI does not have to be transmitted, which yields low latency and high capacity. In addition, more power can be allocated to the Orthogonal Frequency Division Multiplexing (OFDM) subchannels with higher channel gain. Schemes with estimation at the receiver side only experience higher outage probability with fast fading channels but have lower complexity.

As the number of BS antennas goes up, the time required to transmit the downlink pilot symbols increases. In addition, as the number of BS antennas grows, FDD channel estimation becomes almost impossible and a TDD approach can resolve this issue. In TDD systems, due to channel reciprocity, only CSI for the uplink needs to be estimated. In addition, linear MMSE based channel estimation can provide near-optimal performance with low complexity [87].

Tables 2.6, 2.7, and 2.8 compare various channel estimation techniques.

\subsection{OFCDM Systems}

In our system model, we have used OFCDM for the link between base stations and users and used OFDM for the link between large and small cell tier base stations due to the following reasons:

- The traditional systems previously used MIMO in combination with one dimensional access techniques as, TDMA allocating all subcarriers to a user for a certain amount of time, FDMA allocating each user a particular subcarrier for transmission (each user will use a different frequency), CDMA assigning a unique code to each user, then they all share a spectrum of various frequencies, and OFDM for higher data rates, which is a combination between transmission of different signals over multiple BS antennas, and dividing the transmission channel into a number of sub channels each using a certain frequency in frequency domain, and into various transmission slots in time domain, so the channel will be composed of frequency-time blocks. OFDM has many drawbacks. When the same subcarriers are used in adjacent cell, this leads to intra cell interference. Also, it does not have coherence frequency diversity. 
Table 2.6: Various channel estimation techniques.

\begin{tabular}{|c|c|c|c|c|c|}
\hline Ref. & $\begin{array}{c}\text { Channel } \\
\text { Estimation }\end{array}$ & System Type & $\begin{array}{c}\text { User } \\
\text { Antennas }\end{array}$ & Channel Type & $\begin{array}{c}\text { Performance } \\
\text { Metric }\end{array}$ \\
\hline $\begin{array}{l}{[88],} \\
2013\end{array}$ & $\begin{array}{l}\text { Compressive } \\
\text { Sensing-Based }\end{array}$ & $\mathrm{MU}$ & Single & $\begin{array}{c}\text { TDD , Flat-Fading } \\
\text { Quasi-Static }\end{array}$ & Estimation Error \\
\hline $\begin{array}{l}{[89],} \\
2013\end{array}$ & $\begin{array}{l}\text { Direction of } \\
\text { Arrival } \\
\text { Estimation }\end{array}$ & $\mathrm{MU}$ & Single & TDD, Ray Vectors & $\begin{array}{l}\text { Mean Square } \\
\text { Errors and } \\
\text { Capacity Loss }\end{array}$ \\
\hline $\begin{array}{r}{[90],} \\
2014 \\
\end{array}$ & $\begin{array}{c}\text { Semi-Orthogonal } \\
\text { Pilot-Assisted } \\
\end{array}$ & $\mathrm{MU}$ & Single & TDD, Rayleigh & $\begin{array}{c}\text { Overall Achievable } \\
\text { Rates }\end{array}$ \\
\hline $\begin{array}{l}{[91],} \\
2014\end{array}$ & $\begin{array}{c}\text { Closed-Loop } \\
\text { Beam Alignment }\end{array}$ & Single User & Single & FDD , Gaussian & Beamforming Gain \\
\hline $\begin{array}{r}{[92],} \\
2014\end{array}$ & Discriminatory & Two-Users & Multiple & $\begin{array}{l}\text { TDD, Rayleigh } \\
\text { Flat Fading }\end{array}$ & $\begin{array}{c}\text { Power and Mean } \\
\text { Squared } \\
\text { Error (MSE) }\end{array}$ \\
\hline $\begin{array}{r}{[93],} \\
2014\end{array}$ & $\begin{array}{c}\text { Low-Complexity } \\
\text { Polynomial }\end{array}$ & Single User & Multiple & $\begin{array}{l}\text { TDD, Quasi-Static } \\
\text { Flat-Fading }\end{array}$ & MSE \\
\hline $\begin{array}{l}{[94],} \\
2014\end{array}$ & $\begin{array}{c}\text { Distributed } \\
\text { Compressive } \\
\text { Channel State } \\
\text { Information at } \\
\text { the Transmitter } \\
\text { (CSIT) }\end{array}$ & MU & Multiple & FDD, Quasi-static & CSI MSE \\
\hline $\begin{array}{r}{[95],} \\
2014\end{array}$ & $\begin{array}{c}\text { Linear } \\
\text { Estimation }\end{array}$ & $\mathrm{MU}$ & Single & $\begin{array}{c}\text { TDD, Narrow band } \\
\text { Memoryless }\end{array}$ & $\begin{array}{c}\text { Residue and Error } \\
\text { Norms }\end{array}$ \\
\hline $\begin{array}{r}{[96],} \\
2014\end{array}$ & $\begin{array}{c}\text { Improved } \\
\text { Multi-cell MMSE }\end{array}$ & $\mathrm{MU}$ & Single & TDD, Rayleigh & MSE \\
\hline
\end{tabular}


Table 2.7: Various channel estimation techniques (Cont. 1)

\begin{tabular}{|c|c|c|c|c|c|}
\hline Ref. & $\begin{array}{c}\text { Channel } \\
\text { Estimation }\end{array}$ & System Type & $\begin{array}{c}\text { User } \\
\text { Antennas }\end{array}$ & Channel Type & $\begin{array}{c}\text { Performance } \\
\text { Metric }\end{array}$ \\
\hline $\begin{array}{r}{[96],} \\
2014\end{array}$ & $\begin{array}{c}\text { Improved } \\
\text { Multicell MMSE }\end{array}$ & MU & Single & TDD, Rayleigh & MSE \\
\hline $\begin{array}{r}{[97],} \\
2014\end{array}$ & CSIT & $\mathrm{MU}$ & Multiple & FDD, Quasi-static & $\begin{array}{c}\text { CSI Mean Squared } \\
\text { Error }\end{array}$ \\
\hline $\begin{array}{r}{[98],} \\
2014\end{array}$ & $\begin{array}{c}\text { Spectrum-Efficiency } \\
\text { Parametric }\end{array}$ & $\mathrm{MU}$ & Single & $\begin{array}{l}\text { FDD, Rayleigh } \\
\text { Fading }\end{array}$ & MSE \\
\hline $\begin{array}{r}{[99],} \\
2015\end{array}$ & Blind & MU & Single & TDD, NA & MSE \\
\hline $\begin{array}{l}{[100]} \\
2015\end{array}$ & $\begin{array}{c}\text { Gaussian-Mixture } \\
\text { Bayesian } \\
\text { Learning }\end{array}$ & MU & Single & TDD, NA & $\begin{array}{c}\text { MSE and Average } \\
\text { User Rate }\end{array}$ \\
\hline $\begin{array}{l}{[101]} \\
2015\end{array}$ & Subspace-Based & $\mathrm{MU}$ & Single & $\begin{array}{c}\text { TDD, Narrow band } \\
\text { Flat Fading }\end{array}$ & $\begin{array}{c}\text { Bit Error Rate and } \\
\text { Eigen Value } \\
\text { Clusters }\end{array}$ \\
\hline $\begin{array}{l}{[102]} \\
2015\end{array}$ & $\begin{array}{c}\text { Simple Discrete } \\
\text { Fourier } \\
\text { Transform } \\
\text { (DFT)-Aided } \\
\text { Spatial Basis } \\
\text { Expansion }\end{array}$ & MU & Single & TDD, Flat Fading & $\begin{array}{l}\text { The Average } \\
\text { Achievable Sum } \\
\text { Rate and MSE }\end{array}$ \\
\hline $\begin{array}{l}{[103]} \\
2015\end{array}$ & $\begin{array}{c}\text { Bayes-Optimal } \\
\text { Joint }\end{array}$ & $\mathrm{MU}$ & Single & $\begin{array}{c}\text { TDD, Flat Block } \\
\text { Fading }\end{array}$ & $\begin{array}{c}\text { Symbol Error Rate } \\
\text { and MSE }\end{array}$ \\
\hline $\begin{array}{l}{[104]} \\
2015\end{array}$ & $\begin{array}{l}\text { Adaptive } \\
\text { Semi-Blind }\end{array}$ & $\mathrm{MU}$ & Single & NA, TDD & Capacity and MSE \\
\hline
\end{tabular}


Table 2.8: Various channel estimation techniques (Cont. 2)

\begin{tabular}{|c|c|c|c|c|c|}
\hline Ref. & $\begin{array}{c}\text { Channel } \\
\text { Estimation }\end{array}$ & System Type & $\begin{array}{c}\text { User } \\
\text { Antennas }\end{array}$ & Channel Type & $\begin{array}{c}\text { Performance } \\
\text { Metric }\end{array}$ \\
\hline $\begin{array}{l}{[105]} \\
2015\end{array}$ & Imperfect & Single User & Single & $\begin{array}{c}\text { TDD, Spatially } \\
\text { Correlated }\end{array}$ & BER \\
\hline $\begin{array}{l}{[106]} \\
2015\end{array}$ & $\begin{array}{c}\text { Atomic Norm } \\
\text { Denoising-Based }\end{array}$ & MU & Single & $\begin{array}{c}\text { TDD, Flat-Fading } \\
\text { Quasi-Static }\end{array}$ & Estimation Error \\
\hline $\begin{array}{l}{[107]} \\
2016\end{array}$ & $\begin{array}{c}\text { Structured } \\
\text { Compressive } \\
\text { Sensing-Based } \\
\text { Spatio-Temporal }\end{array}$ & Single User & Single & $\begin{array}{c}\text { FDD, Fast } \\
\text { time-varying }\end{array}$ & $\begin{array}{c}\text { Mean Squared } \\
\text { Error, BER, and } \\
\text { Average } \\
\text { Throughput }\end{array}$ \\
\hline $\begin{array}{l}{[108],} \\
2016\end{array}$ & Bayes & $\mathrm{MU}$ & Single & $\begin{array}{l}\text { TDD, Flat block } \\
\text { fading }\end{array}$ & $\begin{array}{l}\text { MSE, and Symbol } \\
\text { Error Rate (SER). }\end{array}$ \\
\hline $\begin{array}{l}{[109]} \\
2017\end{array}$ & $\begin{array}{c}\text { Eigenvalue } \\
\text { Decomposition }\end{array}$ & $\mathrm{MU}$ & Single & TDD, Fast fading & $\begin{array}{c}\text { Rate Loss, and } \\
\text { SER }\end{array}$ \\
\hline $\begin{array}{l}{[110]} \\
2017\end{array}$ & Beam-Blocked & $\mathrm{MU}$ & Single & $\begin{array}{c}\text { FDD, Spatio } \\
\text { Correlation } \\
\text { Channel }\end{array}$ & $\begin{array}{c}\text { MSE, Achievable } \\
\text { Rate, and } \\
\text { Reconstruction } \\
\text { SNR }\end{array}$ \\
\hline $\begin{array}{l}{[111]} \\
2017\end{array}$ & $\begin{array}{c}\text { Joint Angle-Delay } \\
\text { Subspace }\end{array}$ & $\mathrm{MU}$ & Single & $\begin{array}{l}\text { TDD, Flat Rich } \\
\text { Scattering }\end{array}$ & $\begin{array}{c}\text { Projection Error } \\
\text { Power, Path } \\
\text { Number, and MSE }\end{array}$ \\
\hline $\begin{array}{l}{[112],} \\
2017\end{array}$ & Beam-Domain & $\mathrm{MU}$ & Multiple & TDD, Block-fading & $\begin{array}{l}\text { Mean Squared } \\
\text { Error, and BER }\end{array}$ \\
\hline $\begin{array}{l}{[113]} \\
2017\end{array}$ & $\begin{array}{c}\text { Low Rank } \\
\text { Covariance } \\
\text { Matrix }\end{array}$ & $\mathrm{MU}$ & Single & $\begin{array}{c}\text { TDD, Flat } \\
\text { Rayleigh fading }\end{array}$ & NMSE \\
\hline
\end{tabular}


- OFCDM offers two dimensional spreading in time and frequency domains, this resulted in a jump in mean data rate, flexible transmission rates, and increase in frequency diversity gain. In OFCDM the data signal is spread using a time-domain code, this signal is duplicated in frequency-domain, and finally, multiplied by the frequency-domain spreading code. A maximum of eight codes are available per channel, hence various code channels are assigned to a user, which provides variable degrees of freedom for data rate.

- OFCDM offers spreading in time and frequency domains to increase data rate, and frequency diversity gain. Combining OFCDM with massive MIMO takes the advantages of both systems together. Also, OFCDM enables the transmission of different types of traffic, or multi-class traffic, as it provides up to eight code channels. Every class of users will have a unique code. We used in our model three types of users (data, video, and audio users each one with a different data rate).

- OFCDM supports multiple classes of UEs up to eight classes, where every class has its own unique code. Multiple classes means that a UE will a user can transmit voice, data, video,....etc all at the same time.

- OFCDM provides less BER as shown in Fig. 2.2.

Fig. 2.2 shows BER performance for $u$ users communicating with their associated BS for 128 subcarriers with various configurations, where configuration 1 represents a spreading factor in time domain $\mathrm{N}=32$, number of users $u=64$, configuration 2 represents $\mathrm{N}=16, u=32$, and configuration 3 represents $\mathrm{N}=8$ and $u=32$. We can see that increasing the spreading factor decreased BER for the same number of users.

Fig. 2.3 highlights the difference between OFDM-TDMA and OFCDM-TDMA concepts. This system will enable transmission of large number of users in each user class. In [114] the authors introduce the new OFCDM system with two-dimensional spreading for high speed communication for future $5 \mathrm{G}$ networks. Also, the authors discuss the hybrid Multi Code Interference Cancellation (MCI), and MMSE detection algorithm. Also, the availability of applying turbo-coding and MIMO for OFCDM is discussed as well and compared to OFDM. MIMO-OFCDM with multi-code transmission is employed in [115]. The authors investigate MIMO-OFCDM with multi-code transmission in the presence of co-channel interfering signals, and also, multi code interference from other code 


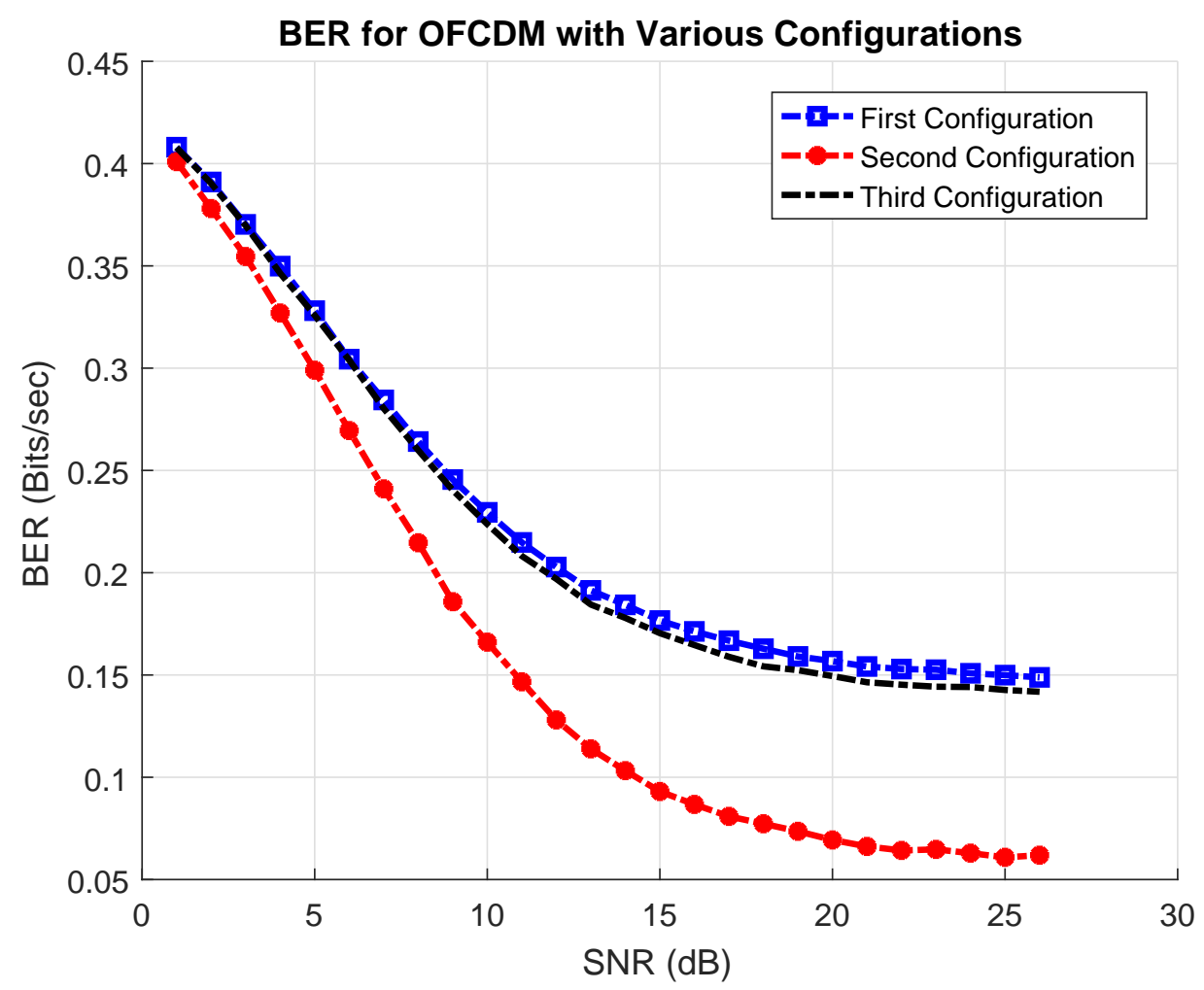

Figure 2.2: BER for OFCDM System with Various Configurations.

channels on the same antenna. They proposed that the combination of iterative detection, the hybrid MCI cancellation, and MMSE detection cancels out multi-antenna interference, and multi-code interference increases frequency diversity, which improves system performance in general. Also, in [116] the authors investigate MIMO-OFCDM, and propose the combination of two detection algorithms in the space and frequency domains to improve diversity gain in space and frequency. Iterative detection in the space domain and the hybrid multi-code interference cancellation, and MMSE detection in the frequency domain. Also for channel estimation they used a two-dimensional averaging algorithm. Moreover, they derived the optimal power allocation between the pilot and all data channels, and it was shown the the optimal power ratio depends on the number of transmit antennas, and the used channel estimation method. Besides, authors in [117] investigate the power allocation in MIMO-OFCDM. Applying minimization of the MSE during signal detection, the optimal power ratio between the pilot and data channels was derived. It was proved that the optimal power ratio is sensitive to the number of transmit antennas and the number of pilot and data symbols in a packet. 


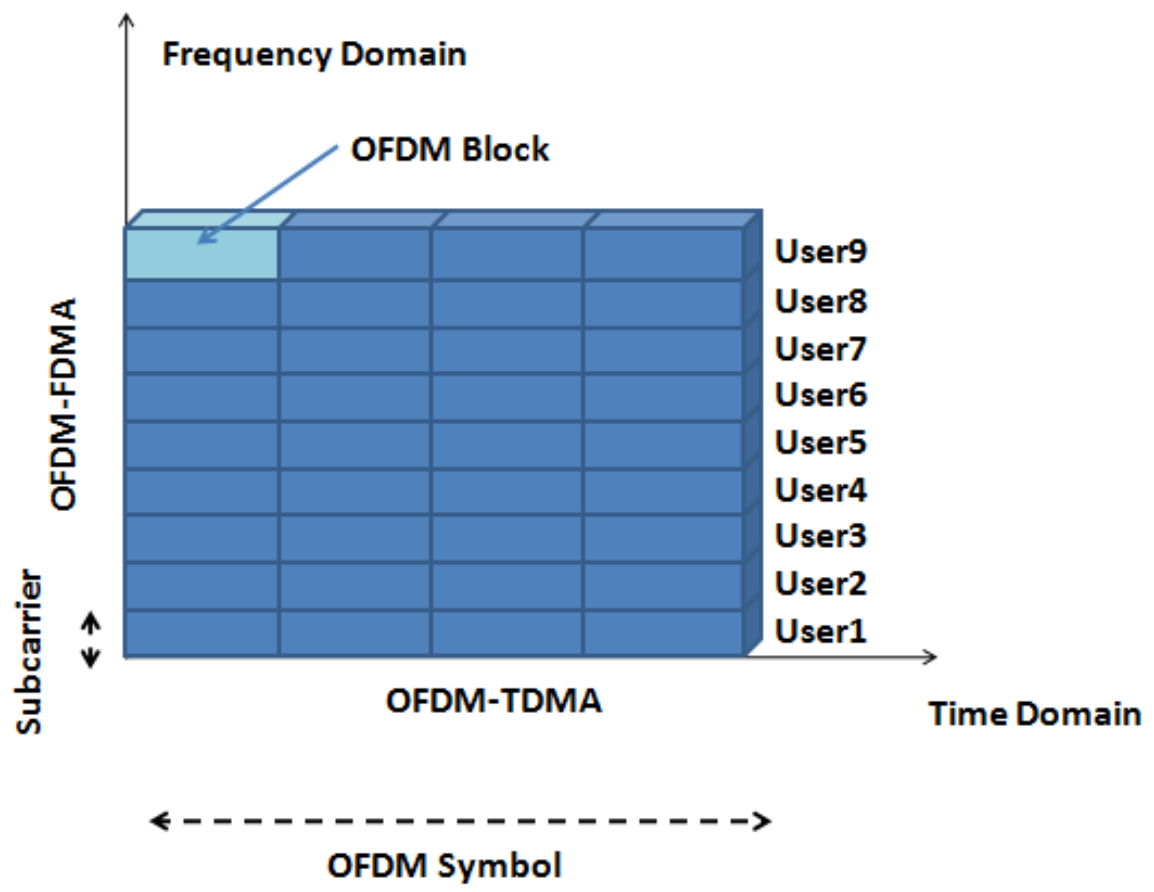

(a) OFDM-TDMA signal structure.

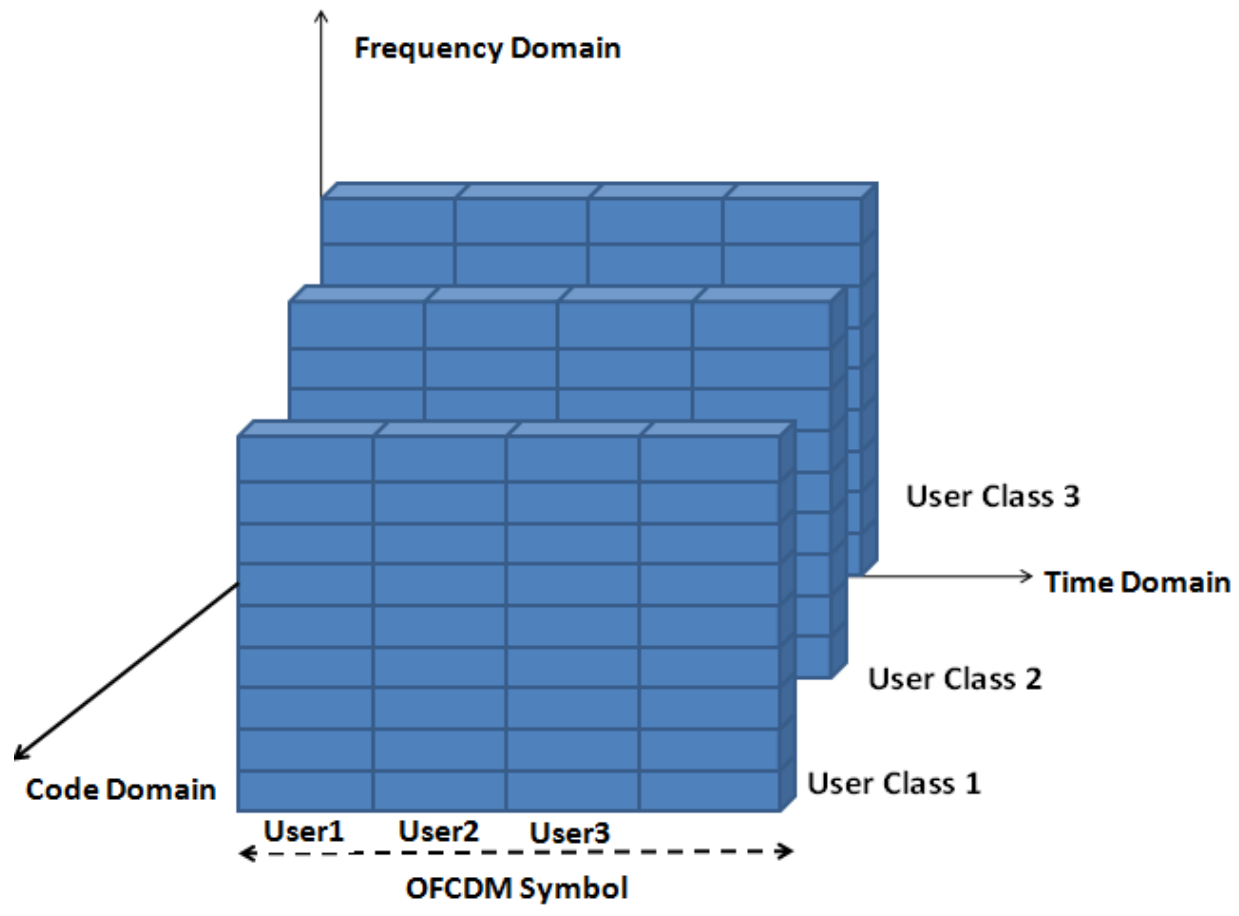

(b) OFCDM-TDMA signal structure.

Figure 2.3: OFDM-TDMA vs OFCDM-TDMA comparison. 


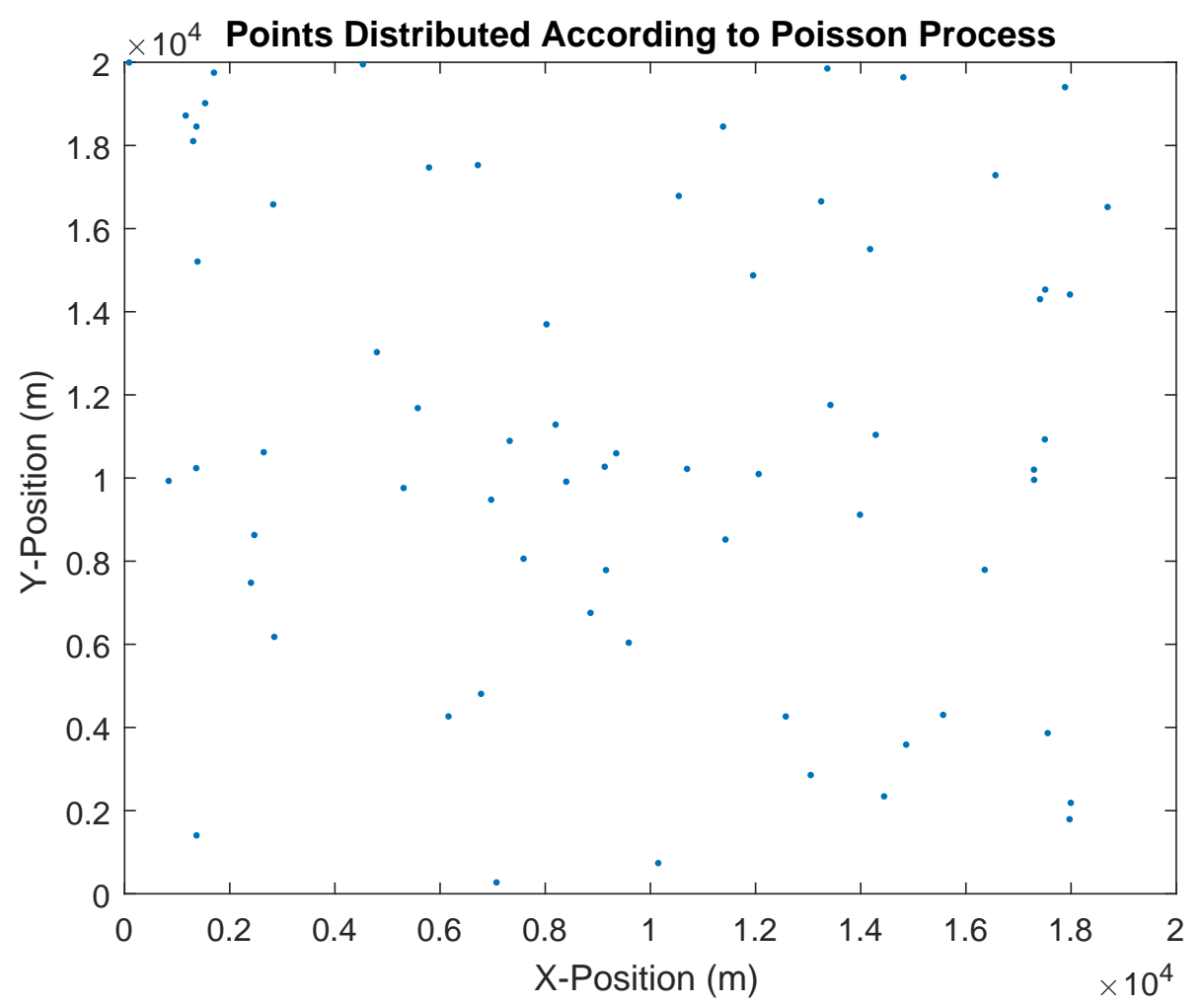

Figure 2.4: Poisson point process with $\lambda=55$, and thinning distance less than $200 \mathrm{~m}$.

In [118], the authors introduce analytical derivations to determine the performance of OFCDM systems with multi-code transmissions taking correlation of the error events on multiple code channels into account. They assumed the utilization of the combined detection techniques of Zero Forcing Successive Interference Cancellation (ZF-SIC) in the space domain, and MMSE detection in the frequency domain. Authors in [119] discuss the performance of OFCDM with two dimensional spreading and multi-code transmission, where time-multiplexed pilot signals are used for channel estimation. Conditional bit error rate is evaluated. The proposed approach showed promising results in various channel estimation qualities and channel correlation conditions. Finally, in [120] the authors introduced optimization of two dimensional spreading in OFCDM for subcarrier allocation in femto/macro hybrid networks. An expression of signal to noise ratio for femto and macro users is derived. Varying the load conditions and the spreading factors bit error rate was obtained as well. 


\subsection{Poisson Point Process}

Along this dissertation, we will use a Poisson point process to deploy base stations and users in the network. A Poisson point process is a random variable that consists of randomly (uniformly) located on a mathematical space used to model certain random events happening in time like scattered users in a wireless network. Each point is stochastically independent of all other points in the process. Fig. 2.4 shows the uniformly distributed points according to a Poisson point process. Every Poisson point process is parameterized by the intensity $\lambda$ for which the number of generated points has a Poisson distribution. If $\lambda$ is a constant, then the process is homogeneous, otherwise it is inhomogeneous or that the probability of generating a point varies in time with a rate function $\lambda(t)$. For the Poisson distribution, the probability of a Poisson random variable $N$ is equal to $n$ is:

$$
P(N=n)=\frac{\lambda^{n}}{n !} e^{-\lambda}
$$

A thinning to the process implies two steps, where a set of locations are generated as a simple Poisson point process with intensity $\lambda$, those locations occur more frequently than the process itself, then throw out some of them and accept the others.

\subsection{Description of System Model in this Dissertation}

In this Section, we explain the network model we use in this dissertation. The multi-tier dense system located on the Two Dimensional (2D) Euclidean plane is presented clearly in Fig. 2.5, where $K$ tiers of BSs are indexed by the set $k=\{1,2,3, \ldots . K\}$. We consider three types of links in this work; Macro-Micro backhaul links, Macro-UE direct links, and Micro-Micro access links. There are two levels of communication considered in our system; one is between the Macro and the Micro BSs, and the other is between the Macro or Micro BSs and their associated UEs. The system is assumed to be open access and BSs in every tier are distributed according to a PPP $\phi_{k}$ with density $\lambda_{k}$. The system is allocated with a total of $M$ Macro BSs having $B_{n}$ hundreds of antennas each(massive MIMO). The square working space has an area of $A$. Also, in each cell there are $C$ Micro BSs each with $A_{n}$ available MIMO antennas relaying data to and from the associated UEs to the Macro BS. The minimum allowed distance between Micro BSs is $d_{m i n}$ and the minimum allowed distance between a Macro BS and its associated Micro BS in the same cell is $d_{c p}$ Every BS is associated 
with $U$ UEs with density $\lambda_{u} . P_{u}$ is the transmit power from UEs and is equal for all UEs. We assume that there is a downlink flow from BSs to their associated UEs. Every UE transmits $S$ data streams at a time. $E_{b}$ is the bit energy and $E_{c}$ and $T_{c}$ are the chip energy and the chip duration respectively. The communication between various tier BSs (Macro to Micro BSs) is using an OFDM system, where each BS schedules transmission over $T$ time-frequency slots. The communication links between BSs and UEs are through an OFCDM system, where the spectrum is divided into groups and each with $Z$ equally spaced subcarriers. Subcarriers are divided equally among groups and every UE can use one group per time for transmission. Groups are denoted by $G_{d}$ with group index denoted as $d=1,2,3, \ldots . D$. TDMA is used in transmitting the signal and the transmitted signal is spread with Pseudo Noise (PN) sequences in time domain with $N$ spreading factor and the signal is spread in frequency domain with $F$ spreading factor. Also, We assume TDD operation from the BS to its associated UEs, so that every BS can estimate the downlink channel coefficient from the uplink pilot transmitted by the UE using channel reciprocity of the CSI. $N_{b}$ is the number

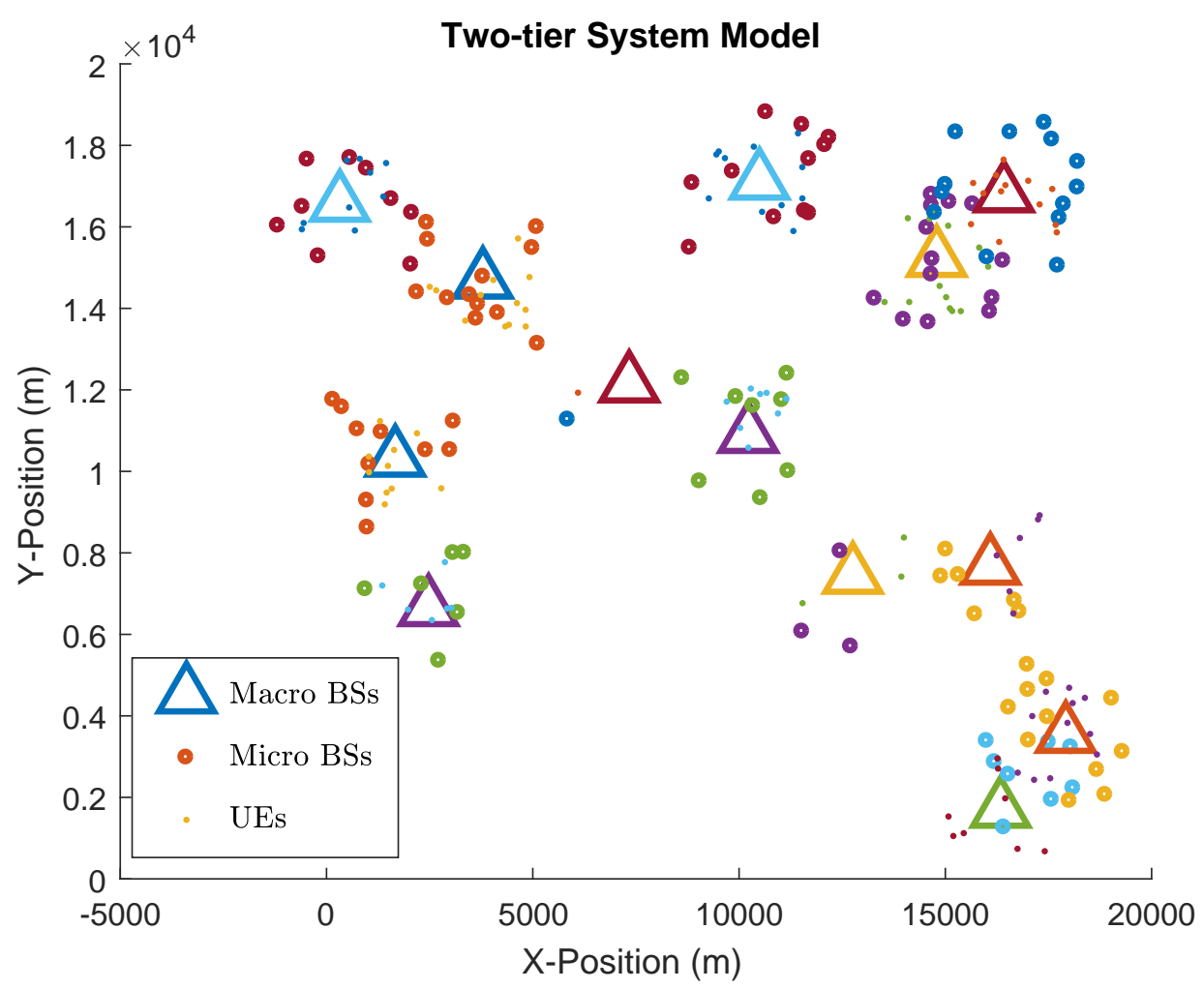

Figure 2.5: An illustration of a PPP-based two-tier cellula network deployment of $20 \mathrm{~km} \times 20 \mathrm{~km}$ area, $\lambda_{1}=15$, and $\lambda_{2}=10$. 


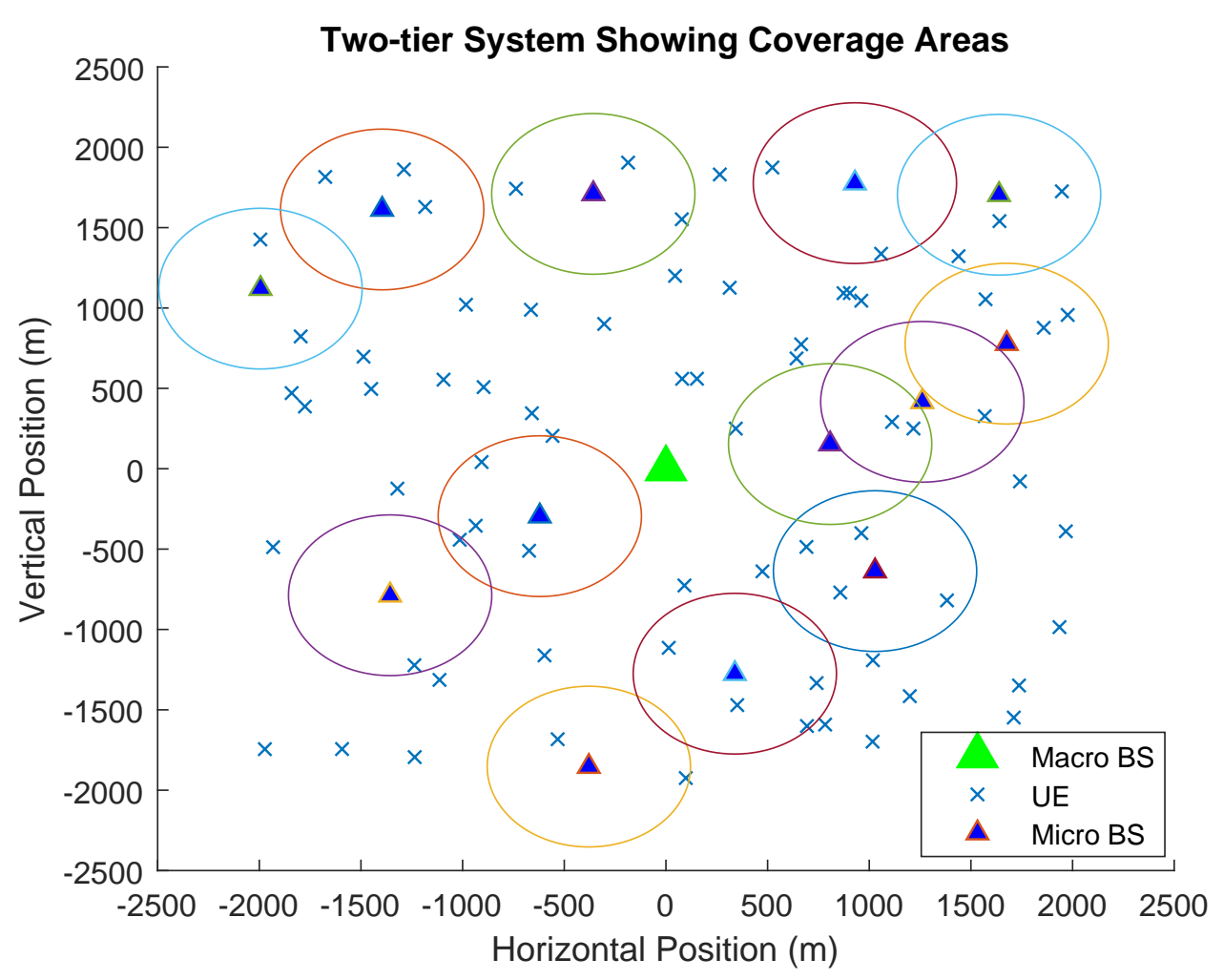

Figure 2.6: An illustration of a two-tier HetNet model of coverage area $(5000 \mathrm{~m} \times 5000 \mathrm{~m}), \lambda_{k}=20$, $\lambda_{u}=80, \mathrm{r}=500 \mathrm{~m}$, and $d_{\text {min }}=200 \mathrm{~m}$.

of transmitted bits, and $E_{b}$ is the bit energy. the transmitted signal is spread with PN sequences in time domain with chip energy $E_{c}$ and chip duration $T_{c}$ where $E_{c}=E_{b} /(N \cdot F)$. Users in the uplink are using nonorthogonal pilot sequences $x_{j}$, where the main Micro BS is at cell $j$ and the other Micro BSs are at $L$ interfering cells All Micro BSs use the same sub channels and transmit on the same frequencies, and UEs in neighboring cells use the same non-orthogoal pilot sequences, so pilot contamination affects this system.

If we zoom in into Fig. 2.5, we get the details of one Macro cell along with its associated Micro BSs and UEs as shown in Fig. 2.6 The system is assumed to be open access, where there is no restriction on the association of UEs to a certain tier BS. Interference is the major limiting parameter that affects network performance. In our model, we consider block-fading channel model with large and small-scale fading [121]. The large-scale fading is a function of distance, path loss attenuation, and multi-path fading components. The small-scale fading coefficients are Rayleigh. The path loss exponent for the Macro and Micro tiers is considered the same as $\alpha$. BSs are assumed to be 
cooperating and exchanging their status between each other (whether they are accepting or giving UEs). Traffic transfer between BSs is enabled from heavily loaded BSs to lightly loaded BSs and vice versa.

The channel impulse response from the Macro BS to the different Micro BSs on subcarrier $T$ will be:

$$
\mathbf{h}_{\mathbf{m}, \mathbf{c}}^{(\mathbf{T})}=\left(\left|\mathbf{h}_{\mathbf{m}, \mathbf{c}}^{(\mathbf{T})}\right|\right) \mathbf{e}^{\mathbf{j} \phi_{\mathbf{m}, \mathbf{c}}}
$$

where $\mathbf{h}_{\mathbf{m}, \mathbf{c}}$ is the small-scale fading gain vector between the Macro BS and the various Micro BSs equals to $\mathbf{h}_{\mathbf{m}, \mathbf{c}}=\left[\mathbf{h}_{\mathbf{m}, \mathbf{1}, \mathbf{c}}, \ldots, \mathbf{h}_{\mathbf{m}, \mathbf{B}_{\mathbf{n}}, \mathbf{c}}\right]^{\mathbf{T}}$ and $\phi_{\mathbf{m}, \mathbf{c}}$ is its phase component.

\subsection{Dissertation Motivation}

Due to many challenges that face optimal design of multi-tier HetNets, our main goal was to enhance the performance of such systems by trying to find new methods to mitigate high interference levels in such ultra dense closely packed systems and to suggest new UE association algorithms to provide fair load balance among different tiers, as the conventional max-Signal to Interference and Noise Ratio (SINR) and max-Received Signal Strength (RSS) algorithms do not provide adequate results in more than one tier systems.

Channel estimation using known pilot sequences is crucial for detecting Channel State Information (CSI) at the beginning of every coherence time interval. Optimum pilot length depends on the parameter to be optimized. We optimized pilot sequence length using mean squared error constraint, which is the most important parameter to reduce channel estimation error. We also considered pilot power constraint to keep it below a certain limit so as not to affect data transmission. Pilot length should be at least equal to the number of users (with at least one pilot per user). Our optimum pilot length depends on number of users so our results are considered feasible and acceptable. Our algorithm is simple with no complexities in implementation. Interference level is very high in dense HetNets. We reduce interference level by altering locations of Micro and Macro BSs and optimizing them. It is very important to properly deploy BSs to improve coverage, and reduce interference level. BSs in HetNets are normally deployed according to a Poisson point process, which may not be the best locations to achieve the best performance. We optimize Micro BSs locations to improve coverage and reduce interference. Also, we were able to isolate Macro cells from all sources 
of interference and consider them as independent units, which will enable any further study to users and Micro BSs associated with every Macro cell without considering inter-cell interference. Conventional user association algorithms do not provide adequate performance in HetNets due to the nature of HetNets (variable BSs transmission power). We propose two user association algorithms to balance load in HetNets. The first algorithm (Least Standard Deviation (LSTD)), works by minimizing network load standard deviation, where UE attempts to connect to the BS with the least load. Network standard deviation is rechecked after every change in any BS load until a balanced load distribution is obtained. We initially start from acceptable values of SINR as load balance is not separated from SINR calculation because it is a crucial factor in determining the validity of associating a user to a certain BS and not choosing another one instead. This algorithm is new and nobody has pointed to it before. Standard deviation is a strong statistical measure of how far a certain distribution is deviated from the mean value that is why it was our chosen method. The second algorithm (Real Time Load Balancing (RTLB)) is a dynamic fast UE association algorithm, which is based on the assumptions that BSs are exchanging their load information among each other (whether they are giving, accepting, or fully loaded) and that traffic transfer is possible from one BS to another. The algorithm considers UEs mobility (as UEs change their position) and status

change (when new calls are established or when calls are dropped or naturally terminate). Our algorithm is simple and based mainly on SINR value for every user. It is fast, because we only process the change and associate newly added users or users that changed location only in every iteration. Furthermore, we derive coverage probability of moving UEs considering handover and no handover scenarios. Both suggested algorithms provide a fair load distribution, however RTLB outperforms the performance of LSTD in real time scenarios as it easily and quickly adapts to rapid network changes.

\subsection{Dissertation Contributions}

The main contributions of this work are to study the problem of interference mitigation in ultra dense small cell HetNets by redeploying Micro and Macro BSs starting initially from the locations obtained from PPP and moving into better locations to improve the performance. Also, we develop two user association algorithms designed specially for HetNets to provide fair load distribution 
among various tiers with variable BSs transmission powers.

- We optimize the pilot sequence length by using the total power of the training period and the MSE of the channel as a constraint. The optimum pilot sequence depends on the number of users. Our approach is simple, yet useful in obtaining an expression of the optimum pilot length.

- We introduce an efficient method for deploying Micro BSs in a two-tier HetNet system to maximize SINR for the uplink channel between Micro and Macro BSs. We proved that signal power was increased by about $2 \mathrm{~dB}$.

- We provide analytical derivations for coverage probability and network energy efficiency and compare the performance before and after optimization through extensive analysis and simulation results.

- We manipulate the derived expression of coverage probability to formulate a constrained deployment algorithm for Micro BSs locations by minimizing inter-cell interference and maximizing the cell coverage. Also, We compare our results with other work done and show that our performance shows better promising results.

- We develop a new algorithm to find the optimum locations of Macro BSs in HetNets where every Macro BS along with its associated Micro BSs is treated as an independent unit after almost eliminating interference from neighboring Macro and Micro BSs. Hence, this approach provides increased coverage area and enhanced quality of service. Simulation results show the validity of our approach and promises enhancement to system performance.

- We develop two user association algorithms. The first one is for associating UEs to BSs based on minimizing the network load standard deviation which restores and maintains the load balance at every BS in the network. Also, we develop a new real-time dynamic fast UE association algorithm for multi-tier cooperating systems that considers users' mobility and traffic dynamics (when number of active UEs at a given instance is continuously changing). Our new algorithms are studied and analyzed through simulation and they proved to provide the best performance compared to other algorithms. 


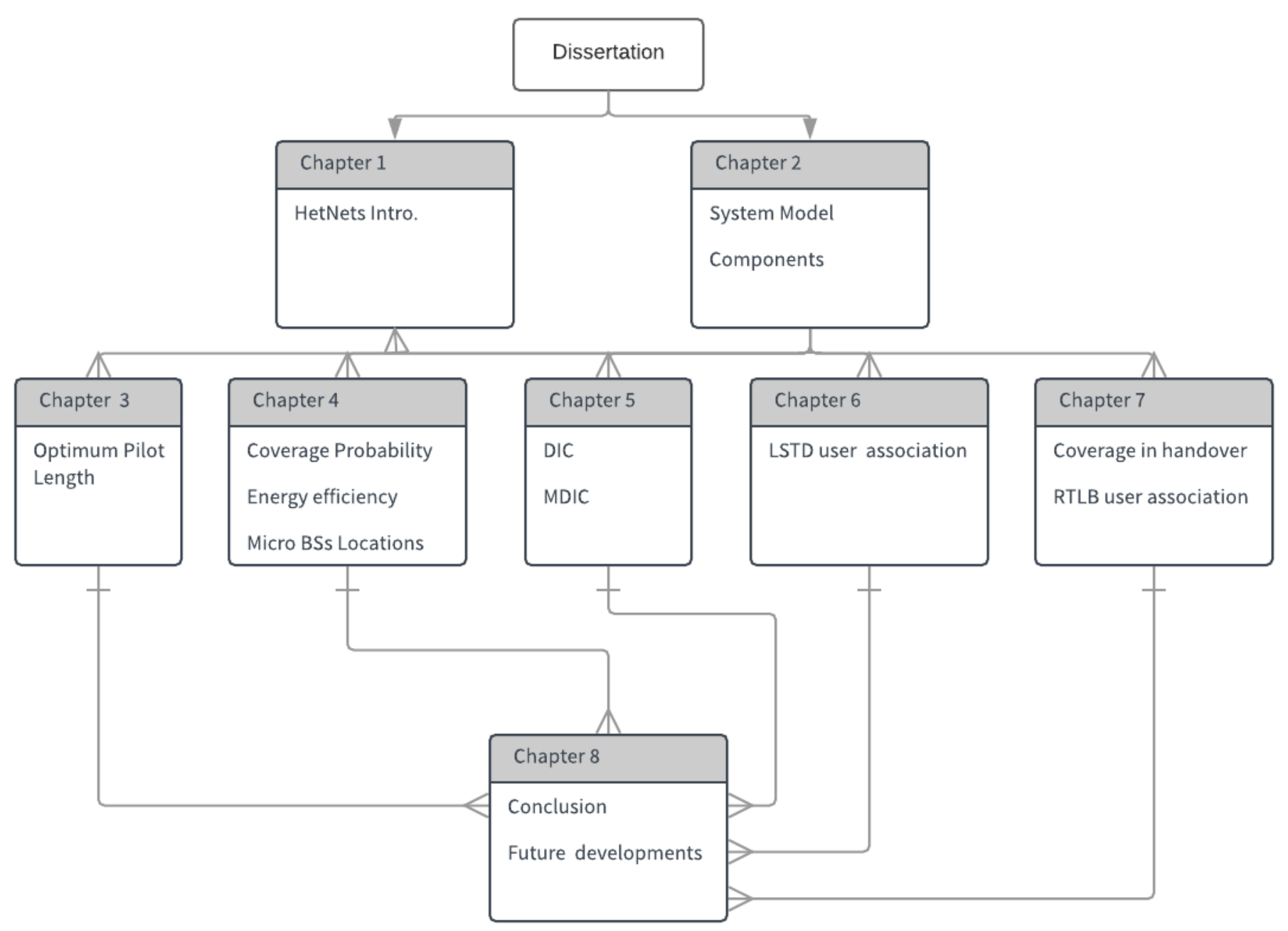

Figure 2.7: Summary of dissertation outline.

\subsection{Dissertation Outline}

The work reported in this dissertation is divided into eight chapters. Fig. 2.7 presents the summary of the outline. The content of the dissertation chapters is briefly described as follows:

- Chapter 1 presents a general explanation of Homogeneous and Heterogeneous systems, challenges, comparison, and advantages and disadvantages of applying each system. Also, we added some brief discussions about pilot contamination, optimizing BSs locations, and user association to BSs 
- Chapter 2 presents and explains the proposed system model and an explanation of its various components.

- Chapter 3 suggests an analytical optimization problem for pilot sequence length to maximize pilot power and reduce minimum mean squared error.

- In chapter 4, we solve some optimization functions for deploying Micro BSs to minimize interference from adjacent BSs, maximize received signal power, and improve coverage. Also, we develop analytical expressions for coverage probability and energy efficiency.

- In chapter 5, emanating from the idea of grouping Micro BSs into core and edge BSs, we propose new algorithms for optimizing Macro BSs locations by minimizing interference from Macro BSs in adjacent cells, and edge Micro BSs in adjacent cells. Also, we combine this algorithm with optimizing core Micro BSs in every Macro cell to ensure that interference level is less than a certain threshold.

- In chapter 6 , we propose our first user association algorithms for HetNets. The algorithm's main idea is to minimize load standard deviation for all BSs in the network. We sort users in ascending order based on possible BSs available for connection and sort available BSs for every user in ascending order based on the BS load. For a certain user, we start by all combinations and trying all available BSs for that user and for the rest of the users we take the BS with least load. We calculate load standard deviation in every iteration until an acceptable standard deviation is achieved. If system data set changes (new users are added or dropped or users move their position), the algorithm has to run all over again from the beginning. Our algorithm takes about 1 sec to run for every data set change.

- In chapter 7, we propose our second user association algorithms for HetNets. The algorithm's main idea is that BSs share and broadcast their load information between each other and that traffic transfer is possible between BSs (from less loaded to heavily loaded and vice versa). BSs are classified into giving, accepting, and fully loaded BSs according to comparison with a certain threshold. We start from the first giving BS and move the UE having the highest available SINR, then we specify the accepting BSs and move the user to the one with the highest SINR. Some users might change their position, so we have to recalculate SINR for 
them. Also, new users might be added, so we update the number of active users and calculate the new SINR matrix. Some users' connections are terminated as their calls are ended, so they should be dropped from their associated BSs. The algorithm takes 0.02 sec per every data set change.

- Chapter 8, evaluates the achievement of the dissertation compared to the goals and discusses various contributions. We also covered some of the future developments.

\subsection{Chapter Summary}

In this chapter, we studied the system model proposed in this dissertation. Also we defined and explained its various components. We studied uplink and downlink operations that will be used in various parts of this dissertation. We studied TDD systems, the advantages of their use, and compared with FDD systems. Furthermore, we explained OFCDM systems and their advantages over conventional OFDM systems. Also, we gave a brief idea about PPP. We studied HetNets, where

more traffic management, radio resource management, network planning, and inter-tier interference management are required, as those networks are more complicated, have more dense cells, and any user can access any BS in any tier. We observed that fast booming HetNets would be more promising to improve data rates and provide flexibility in user-BS association. Finally, we explained the system model used in this dissertation, listed our contributions, and presented the dissertation outline. 
CHAPTER 3

\section{Optimization of Pilot Sequence Length}

\subsection{Introduction}

In a time-division duplex system, the BS estimates the channel from mutually orthogonal reverse-link pilot sequences within a cell to formulate a receiver for the reverse link and a precoder for the forward link. The channel coherence, as well as frequency, is typically constrained at the time, this leads to an imbalance between the resources spent on pilots and those available for data symbols. Reusing pilot sequences in nearby cells, reduces pilot overhead, however, this potentially increases interference in the channel estimation phase, known as 'pilot contamination' effect [122].

Though long pilot sequences would reduce pilot contamination, they replace data sequences and reduce channel spectral efficiency and throughput [3]. Pilot contamination is combatted using time-shifted pilots, and pilot hopping at each time slot [123], optimizing the precoding matrix [124], by using the angle of arrival methods [5], and finding the optimum pilot reuse factor [125].

In this chapter, we optimize the pilot sequence length by using the total power of the training period, and the estimation error as the constraint. The optimum pilot sequence depends on the number of users. Our approach is simple, yet useful in obtaining an expression of the optimum pilot length. We use Lagrange optimization, which is considered the most accurate analytical method for optimization. For finding the optimum pilot length, we can maximize or minimize the pilot length. Maximizing or minimizing the pilot sequence length is critical in systems design. Minimizing its length improves spectral efficiency, channel capacity, mean squared error, data rate, 
and throughput. Maximizing the pilot sequence length improves channel estimation quality and reduce pilot contamination. Our approach is simple and with low complexity and can be applied to any application.

\subsection{Related Work}

In this section we will review some of the efforts done pilot length optimization field.

In [126] the authors minimized the training sequence length in correlated channels under the estimation error constraint of the covariance matrix. The obtained pilot sequences are short and non-orthogonal. However, the channel estimation error variance does not exceed a certain constant. For this algorithm, the obtained optimum pilot lengths were not realistic and efficient as they were less than half number of users. In [127] the authors optimized the training sequence length and the pilot symbol power allocation. They derived a relationship between the pilot sequence length and the effective SNR, then maximized the effective SNR under a sum energy constraint and a total block length constraint. They derived the expression for the optimal training interval length maximizing the effective SNR and proving its equality to the number of transmit antennas. However the authors did not discuss the effect of this maximization on pilot contamination, and channel estimation quality. In [128] the authors optimized the pilot sequence length for a given coherence block length by maximizing the net ergodic achievable rate. They also showed the dependence of the optimal training length on the backhaul capacity. In [129] the authors considered optimizing the training sequence length for a particular case of frequency-selective fading channels, considering tap gain correlation and spatial correlations at both ends. The optimal training sequence design should minimize the MSE. MSE was derived for the correlated channel and decreased using Lagrange operator. The MSE for the correlated channel is observed to be less than the uncorrelated case.

Table 3.1 compares optimum pilot length obtained using different approaches. Authors in several papers used various optimization functions (mean squared error, signal to noise ratio, ergodic rate, and pilot length) and various constraints (estimation error, pilot length, pilot power, and capacity). Some of the problems were maximization and others were minimization problems. However, all results depended on number of users (or number of antennas on the transmission side) besides one or more additional parameters. 
Table 3.1: Comparison of related pilot length optimization work in literature.

\begin{tabular}{|c|c|c|c|c|}
\hline Ref. & $\begin{array}{c}\text { Optimization } \\
\text { Problem } \\
\end{array}$ & $\begin{array}{c}\text { Optimization } \\
\text { type }\end{array}$ & Constraints & Optimum Length \\
\hline$[126]$ & Pilot length & Minimization & $\begin{array}{l}\text { Covariance of } \\
\text { estimation } \\
\text { error }\end{array}$ & $\begin{array}{l}\text { Less than number of } \\
\text { users. }\end{array}$ \\
\hline$[127]$ & Effective SNR & Maximization & $\begin{array}{l}\text { Sum energy } \\
\text { and Block } \\
\text { length }\end{array}$ & $\begin{array}{c}\text { Equals to number of } \\
\text { users. }\end{array}$ \\
\hline$[128]$ & $\begin{array}{c}\text { Net ergodic } \\
\text { achievable rate }\end{array}$ & Maximization & Pilot length & $\begin{array}{c}\text { Depends on backhaul } \\
\text { capacity and SNR. }\end{array}$ \\
\hline$[130]$ & Effective SNR & Maximization & Pilot length & $\begin{array}{c}\text { Equals to number of } \\
\text { users. }\end{array}$ \\
\hline$[131]$ & $\begin{array}{l}\text { Effective SNR and } \\
\text { channel capacity }\end{array}$ & Maximization & $\begin{array}{l}\text { Pilot energy } \\
\text { and transmit } \\
\text { energy }\end{array}$ & $\begin{array}{l}\text { Depends on channel } \\
\text { memory. }\end{array}$ \\
\hline$[132]$ & $\begin{array}{l}\text { Mean squared } \\
\text { error }\end{array}$ & Maximization & $\begin{array}{c}\text { Mean square } \\
\text { error and } \\
\text { ergodic } \\
\text { capacity }\end{array}$ & $\begin{array}{c}\text { Depends on number of } \\
\text { transmit antennas and } \\
\text { channel length. }\end{array}$ \\
\hline$[133]$ & Estimation error & Minimization & Capacity & $\begin{array}{c}\text { Depends on number of } \\
\text { transmit antennas and } \\
\text { SNR. }\end{array}$ \\
\hline$[134]$ & $\begin{array}{l}\text { Mean squared } \\
\text { error }\end{array}$ & Minimization & $\begin{array}{c}\text { Maximum } \\
\text { transmission } \\
\text { power }\end{array}$ & $\begin{array}{c}\text { Equals to number of } \\
\text { users }\end{array}$ \\
\hline$[135]$ & $\begin{array}{l}\text { Mean squared } \\
\text { error }\end{array}$ & Minimization & $\begin{array}{c}\text { Pilot power } \\
\text { and estimation } \\
\text { error }\end{array}$ & $\begin{array}{c}\text { Depends on number of } \\
\text { transmit antennas }\end{array}$ \\
\hline Our work & Pilot length & Minimization & $\begin{array}{c}\text { Pilot power } \\
\text { and estimation } \\
\text { error }\end{array}$ & $\begin{array}{c}\text { Depends on number of } \\
\text { users. }\end{array}$ \\
\hline
\end{tabular}




\subsection{Contributions and Organization}

In this chapter, we derive an analytical expression for the optimum sequence length maximizing the pilot power and minimizing error. The optimum length depends on number of users. We chose MSE a constraint to reduce error in estimating the channel and keep it below a certain level. Also, we chose pilot power to be less than a certain threshold so as not to over exceed the data power. Optimal pilot length does not have an exact value and it depends according to the parameters chosen and the optimization constraints. However, an acceptable length should be anywhere from number of users to coherence block length. From that, our result is acceptable as it falls within this range. We organize the remainder of this chapter as follows: Section 3.4 presents the problem formulation. Section 3.5 presents the simulation discussion and Section 3.6 concludes this chapter.

\subsection{Pilot Length Problem Formulation}

The total uplink received signal at all the BS antennas for all $U$ users is the sum of signals in cell $j$ and the sum of interfering signals in the $L$ cells [136]:

$$
\left.y_{j_{n}}=\sqrt{p_{u} \tau} \sum_{u=1}^{U}\left(\sum_{n=1}^{A_{n}} \sqrt{\beta_{j j}} \mathbf{h}_{\mathbf{j} \mathbf{j}_{\mathbf{n}}}\right) \mathbf{x}_{\mathbf{j}_{\mathbf{n}}}+\sum_{n=1}^{A_{n}} \sum_{l=1, l \neq j}^{L} \sqrt{\beta_{j l}} \mathbf{h}_{\mathbf{j} \mathbf{l}_{\mathbf{n}}} \mathbf{x}_{\mathbf{j}_{\mathbf{n}}}+n_{j_{n}}\right)
$$

where, $\beta_{j j}$, and $\beta_{j l}$ are non negative constants known as the propagation coefficients of large-scale fading in channel $\mathbf{H}_{\mathbf{j} \mathbf{j}_{\mathbf{n}}} \in \mathbf{C}^{\mathbf{A}_{\mathbf{n}} \times \mathbf{U}}$ and $\mathbf{H}_{\mathbf{j} \mathbf{l}_{\mathbf{n}}} \in \mathbf{C}^{\mathbf{A}_{\mathbf{n}} \times \mathbf{U}}$ respectively where $\mathbf{H}_{\mathbf{j} \mathbf{j}_{\mathbf{n}}}$ is the channel matrix between all $U$ users in cell $j$ and the Micro BS $j . \mathbf{H}_{\mathbf{j} \mathbf{j}_{\mathbf{n}}}$ is equal to $\beta_{j j} \cdot \mathbf{h}_{\mathbf{j} \mathbf{j}_{\mathbf{n}}}$ and $\mathbf{H}_{\mathbf{j} \mathbf{l}_{\mathbf{n}}}$ is the channel matrix between all $U$ users in the interfering cells $l$ and Micro BS $j . \beta_{j j}$ and $\beta_{j l}$ are equal to $d_{j j}^{-\alpha}$, and $d_{j l}^{-\alpha}$ respectively, where $d_{j j}$ represents the distance from a user in cell $j$ to the Micro BS in the same cell, $d_{j l}$ is the distance between the a user in the neighboring cell $l$ and the Micro BS in cell $j$. $\mathbf{h}_{\mathbf{j} \mathbf{j}_{\mathbf{n}}}$, and $\mathbf{h}_{\mathbf{j} \mathbf{l}_{\mathbf{n}}}$ are the $A_{n} \times U$ fast fading matrices from users at cell $j$ and $l$ to BS at $j^{\text {th }}$ cell respectively. $n_{j_{n}}$ is the independent identically distributed complex additive white Gaussian noise with normal probability density function $N\left(0, \sigma^{2}\right)$ of cell $j$. Furthermore, $\mathbf{x}_{\mathbf{j}_{\mathbf{n}}}$ is the pilot sequence vector with length $\tau$ (number of symbols). The value of $\tau$ is determined by optimization. $p_{u}$ is the average transmitted power of UEs in all cells. $\|$.$\| is the L_{2}$ norm or Euclidean norm. 
$H_{j j}$ is the $A_{n} \times U$ channel matrix from user $U$ in cell $\mathrm{j}$ to BS in cell $\mathrm{j}$.

$$
H_{j j}=\left[\begin{array}{ccc}
h_{j j_{1}} & \ldots \ldots & h_{j j_{1} A_{n}} \\
h_{j j_{U_{1}}} & \ldots \ldots . & h_{j j_{A_{n}}}
\end{array}\right]
$$

$H_{j l}$ is the $A_{n} \times U$ neighboring channel matrix from user $U$ in cell $l$ to BS in cell $j$.

$$
H_{j l}=\left[\begin{array}{ccc}
h_{j l_{1}} & \ldots \ldots & h_{j l_{1} A_{n}} \\
h_{j l_{U_{1}}} & \ldots \ldots . & h_{j l_{U_{A_{n}}}}
\end{array}\right]
$$

The uplink SINR, in this case, will be:

$$
S I N R_{j_{n}}=\frac{\sum_{u=1}^{U} \sum_{n=1}^{A_{n}}\left(p_{u} \tau\left(d_{j j}^{-\alpha}\right)\left|\mathbf{H}_{\mathbf{j}_{\mathbf{n}}}\right|\right)}{\sum_{u=1}^{U} \sum_{n=1}^{A_{n}} \sum_{l=1, l \neq j}^{L}\left(\left(d_{j l}^{-\alpha}\right)\left|\mathbf{H}_{\mathbf{j}_{\mathbf{n}}}\right|+\sigma_{j_{n}}^{2}\right)}
$$

From the uplink received signal discussed above, we get the MMSE estimate of the $H_{j l}$ channel as follows:

$$
\hat{H}_{j l_{n}}=\sqrt{p_{u} \tau \beta_{j j}} x_{j_{n}}^{H}\left(I+x_{j_{n}}\left(p_{u} \tau \sum_{l=1}^{L} \beta_{j l}\right)\right)^{-1} x_{j_{n}}^{H} y_{j_{n}}
$$

Let $e$ be the estimation error of $\hat{H}_{j l_{n}}-H_{j l_{n}}$, then its covariance matrix $\left(C_{e}\right)$ will be $\sqrt{p_{u} \tau \beta_{j j}} x_{j_{n}}^{H}(I+$ $\left.x_{j_{n}}\left(p_{u} \tau \sum_{l=1}^{L} \beta_{j l}\right)\right)^{-1}$.

Multiplying the numerator and denominator by $I-x_{j_{n}}\left(p_{u} \tau \sum_{l=1}^{L} \beta_{j l}\right) x_{j_{n}}^{H}$

$$
\begin{gathered}
\hat{H}_{j l_{n}}=\sqrt{p_{u} \tau \beta_{j j}} x_{j_{n}}^{H}\left(I-\frac{x_{j_{n}}\left(p_{u} \tau \sum_{l=1}^{L} \beta_{j l}\right) x_{j_{n}}^{H} y_{j_{n}}}{1+p_{u} \tau \sum_{l=1}^{L} \beta_{j l}}\right) \\
\hat{H}_{j l_{n}}=\sqrt{p_{u} \tau \beta_{j j}}\left(x_{j_{n}}^{H}-\frac{p_{u} \tau \sum_{l=1}^{L} \beta_{j l}}{1+p_{u} \tau \sum_{l=1}^{L} \beta_{j l}} x_{j_{n}}^{H}\right) y_{j_{n}}
\end{gathered}
$$

Taking $x_{j_{n}}^{H}$ as a common factor:

$$
\hat{H}_{j l_{n}}=\frac{\sqrt{p_{u} \tau \beta_{j j}}}{1+p_{u} \tau \sum_{l=1}^{L} \beta_{j l}} x_{j_{n}}^{H} y_{j_{n}}
$$

As we proved in (3.6) that the overall MSE will be equal to $\frac{\sqrt{p_{u} \tau \beta_{j j}}}{1+p_{u} \tau \sum_{l=1}^{L} \beta_{j l}}$. For this equation to be minimum, the denominator has to be greater than a threshold $(\gamma)$.

$$
1+p_{u} \tau \sum_{l=1}^{L} \beta_{j l} \geq \gamma
$$




$$
\begin{gathered}
p_{u} \tau \sum_{l=1}^{L} \beta_{j l} \geq \gamma-1 \\
p_{u} \tau \sum_{l=1}^{L} \beta_{j l}-\gamma \geq-1
\end{gathered}
$$

Finally, this condition will become as:

$$
-p_{u} \tau \sum_{l=1}^{L} \beta_{j l} \leq 1-\gamma
$$

For our optimization problem, we apply the Least-Squared (LS) technique to minimize the difference between the normalized estimated pilot sequence, and the transmitted pilot sequence, considering $A_{n}$ antennas at the Micro base station.

$$
L S=\operatorname{minimize}\left\{\mathbf{E}\left[\left\|\tilde{j_{n}}-x_{j_{n}}\right\|^{2}\right]\right\}=\operatorname{minimize}\left\{\mathbf{E}\left[\left\|y_{j_{n}}-x_{j_{n}}\right\|^{2}\right]\right\}
$$

The LS estimate $\hat{H}_{j j_{n}}$ of channel $H_{j j_{n}}$ is found to be:

$$
\hat{H}_{j j_{n}}=\frac{\sqrt{p_{u} \tau \beta_{j j}}}{1+p_{u} \tau \sum_{l=1}^{L} \beta_{j l}} y_{j_{n}} \cdot x_{j_{n}}^{H}=H_{j j_{n}}+\sum_{l=1, l \neq j}^{L} H_{j l_{n}}+n_{j_{n}}^{\prime}
$$

where, $n_{j_{n}}^{\prime}=\frac{n_{j_{n}} x_{j_{n}}^{H}}{\sqrt{p_{u} \tau}}, n_{j_{n}}^{\prime} \epsilon C N\left(0, \frac{I_{C}}{\sqrt{p_{u} \tau}}\right)$

Hence,

$$
H_{j j_{n}}=\hat{H}_{j j_{n}}-\sum_{l=1, l \neq j}^{L} H_{j l_{n}}-n_{j_{n}}^{\prime}
$$

Substituting (3.13) into (3.1), then $y_{j_{n}}$ can be written as:

$$
\begin{gathered}
y_{j_{n}}=\sqrt{p_{u} \tau} \sum_{u=1}^{U}\left(\sum_{n=1}^{A_{n}}\left(\hat{H}_{j j_{n}} x_{j_{n}}-\sum_{l=1, l \neq j}^{L} H_{j l_{n}} x_{j_{n}}-n_{j_{n}}^{\prime} x_{j_{n}}+\sum_{l=1, l \neq j}^{L} H_{j l_{n}} x_{j_{n}}+n_{j_{n}}\right)\right) \\
y_{j_{n}}=\sqrt{p_{u} \tau} \sum_{u=1}^{U}\left(\sum_{n=1}^{A_{n}}\left(\hat{H}_{j j_{n}} x_{j_{n}}-n_{j_{n}}^{\prime} x_{j_{n}}+n_{j_{n}}\right)\right)
\end{gathered}
$$

Substituting by the value of $y_{j_{n}}$ in equation (3.11), the objective function (f) will be:

$$
f=\mathbf{E}\left[\left\|\sqrt{p_{u} \tau}\left(\left(\hat{H}_{j j_{n}}-n_{j_{n}}^{\prime}\right) x_{j_{n}}+n_{j_{n}}\right)-x_{j_{n}}\right\|^{2}\right] ; \forall u=1: U, n=1: A_{n}
$$

We will simplify the previous equation into the following:

$$
\left.f=\mathbf{E}\left[\| \sqrt{p_{u} \tau}\left(\hat{H}_{j j_{n}} x_{j_{n}}\right)-n_{j_{n}} x_{j_{n}} x_{j_{n}}^{H}+\sqrt{p_{u} \tau} n_{j_{n}}-x_{j_{n}}\right) \|^{2}\right] \forall u=1: U, n=1: A_{n}
$$


As $x_{j_{n}} x_{j_{n}}^{H}$ is a multiplication of a vector and its hermitian equals to $\tau$, then:

$$
\begin{gathered}
\left.f=\mathbf{E}\left[\| \sqrt{p_{u} \tau}\left(\hat{H}_{j j_{n}} x_{j_{n}}\right)-n_{j_{n}} \tau+\sqrt{p_{u} \tau} n_{j_{n}}-x_{j_{n}}\right) \|^{2}\right] \forall u=1: U, n=1: A_{n} \\
f=\mathbf{E}\left[\left\|x_{j_{n}}\left(\sqrt{p_{u} \tau} \hat{H}_{j j_{n}}-1\right)-n_{j_{n}}\left(\tau-\sqrt{p_{u} \tau}\right)\right\|^{2}\right] \forall u=1: U, n=1: A_{n}
\end{gathered}
$$

The expectation of the square of the norm gives the second moment or the variance, and additive noise $n_{j_{n}}$ is independent on estimated channel, then the property that $\operatorname{Var}(\mathrm{u}+\mathrm{v})=\operatorname{Var}(\mathrm{u})+\operatorname{Var}(\mathrm{v})$ will apply to (3.19) as follows:

$$
\begin{gathered}
f=\mathbf{E}\left[\left\|x_{j_{n}}\left(\sqrt{p_{u} \tau} \hat{H}_{j j_{n}}-1\right)\right\|^{2}\right]-\mathbf{E}\left[\left\|n_{j_{n}}\left(\tau-\sqrt{p_{u} \tau}\right)\right\|^{2}\right] \forall u=1: U, n=1: A_{n} \\
f=\operatorname{Tr}\left\{\mathbf{E}\left[\left\|x_{j_{n}}\left(\sqrt{p_{u} \tau} \hat{H}_{j j_{n}}-1\right)\right\|^{2}\right]-\mathbf{E}\left[\left\|n_{j_{n}}\left(\tau-\sqrt{p_{u} \tau}\right)\right\|^{2}\right]\right\} \forall u=1: U, n=1: A_{n}
\end{gathered}
$$

As the value $\left(\tau-\sqrt{p_{u} \tau}\right)$ is a scalar quantity, so we take it out of the expectation operation as follows:

$$
\begin{gathered}
f=\operatorname{Tr}\left\{\mathbf{E}\left[\left\|x_{j_{n}}\left(\sqrt{p_{u} \tau} \hat{H}_{j j_{n}}-1\right)\right\|^{2}\right]-\left(\tau-\sqrt{p_{u} \tau}\right) \mathbf{E}\left[\left\|n_{j_{n}}\right\|^{2}\right]\right\} \forall u=1: U, n=1: A_{n} \\
f=\operatorname{Tr}\left\{\mathbf{E}\left[\left\|x_{j_{n}}\left(\sqrt{p_{u} \tau} \hat{H}_{j j_{n}}-1\right)\right\|^{2}\right]\right\}-\left(\tau-\sqrt{p_{u} \tau}\right) \cdot \operatorname{Tr}\left\{\mathbf{E}\left[\left\|n_{j_{n}}\right\|^{2}\right]\right\} \forall u=1: U, n=1: A_{n}
\end{gathered}
$$

The term $\mathbf{E}\left[\left\|n_{j_{n}}\right\|^{2}\right]$ is equal to $\sigma^{2} I_{u}$ and its trace equals to $\sigma^{2} U$

$$
\begin{gathered}
f=\operatorname{Tr}\left\{\mathbf{E}\left[\left\|x_{j_{n}}\left(\sqrt{p_{u} \tau} \hat{H}_{j j_{n}}-1\right)\right\|^{2}\right]\right\}-\sigma^{2} U\left(\tau-\sqrt{p_{u} \tau}\right) \forall u=1: U, n=1: A_{n} \\
f=\operatorname{Tr}\left\{\mathbf{E}\left[x_{j_{n}} x_{j_{n}}^{H}\left(\sqrt{p_{u} \tau} \hat{H}_{j j_{n}}-I_{u}\right) \cdot\left(\sqrt{p_{u} \tau} \hat{H}_{j j_{n}}-I_{u}\right)^{H}\right]\right\}-\sigma^{2} U\left(\tau-\sqrt{p_{u} \tau}\right) \forall u=1: U, n=1: A_{n}
\end{gathered}
$$

As $x_{j_{n}}$ and $\hat{H}_{j j_{n}}$ are independent, then:

$$
\begin{gathered}
f=\operatorname{Tr}\left\{\mathbf{E}\left[x_{j_{n}} x_{j_{n}}^{H}\right] \cdot \mathbf{E}\left[\left(\sqrt{p_{u} \tau} \hat{H}_{j j_{n}}-I_{u}\right) \cdot\left(\sqrt{p_{u} \tau} \hat{H}_{j j_{n}}-I_{u}\right)^{H}\right]\right\}-\sigma^{2} U\left(\tau-\sqrt{p_{u} \tau}\right) \forall u=1: U, n=1: A_{n} \\
f=\operatorname{Tr}\left\{\tau \cdot \mathbf{E}\left[\left(\sqrt{p_{u} \tau} \hat{H}_{j j_{n}}-I_{u}\right) \cdot\left(\sqrt{p_{u} \tau} \hat{H}_{j j_{n}}-I_{u}\right)^{H}\right]\right\}-\sigma^{2} U\left(\tau-\sqrt{p_{u} \tau}\right) \forall u=1: U, n=1: A_{n}
\end{gathered}
$$$$
f=\tau \cdot \operatorname{Tr}\left\{\left(p_{u} \tau \mathbf{E}\left[\hat{H}_{j j_{n}} \hat{H}_{j j_{n}}^{H}\right]\right)-\left(\sqrt{p_{u} \tau} \hat{H}_{j j_{n}}^{H}\right)-\left(\sqrt{p_{u} \tau} \hat{H}_{j j_{n}}\right)\right\}-\sigma^{2} U\left(\tau-\sqrt{p_{u} \tau}\right) \forall u=1: U, n=1: A_{n}
$$ 


\subsubsection{Asymptotic Analysis}

To perform optimization of the pilot sequence length, we add a power constraint and a MMSE constraint and solve Lagrange equation; the optimization problem will become:

$$
\begin{array}{ll}
\underset{\tau}{\min (f)} & \\
\text { subject to } & C_{1}:-p_{u} \tau \sum_{l=1}^{L} \beta_{j l} \leq 1-\gamma \\
& C_{2}: \tau \geq U \\
& C_{3}: A_{n} \gg U
\end{array}
$$

where $C_{1}$ depicts that MMSE is less than another threshold. $C_{2}$ follows from the fact that pilot sequence length should be at least equal to the number of UEs to provide one value for every UE. $C_{3}$ ensures that number of Micro BS antennas should be so much greater than number of served UEs.

By applying Lagrange multiplier and adding Lagrange operator $\lambda$, the final optimization problem after adding the constraints will be:

$$
L(\tau, \lambda)=L(\tau)+\lambda\left[-p_{u} \tau \sum_{l=1}^{L} \beta_{j l}-(1-\gamma)+\tau-U\right]
$$

From (3.28), the trace of sum is equal to the trace of every individual element then we get:

$$
\begin{array}{r}
L(\tau, \lambda)=\tau^{2} p_{u} \operatorname{Tr}\left\{\left(\mathbf{E}\left[\hat{H}_{j j_{n}} \hat{H}_{j j_{n}}^{H}\right]\right)\right\}-\tau \sqrt{p_{u} \tau} \operatorname{Tr}\left\{\hat{H}_{j j_{n}}^{H}\right\}-\tau \sqrt{p_{u} \tau} \operatorname{Tr}\left\{\hat{H}_{j j_{n}}\right\}-\sigma^{2} U\left(\tau-\sqrt{p_{u} \tau}\right)+ \\
\lambda\left[-p_{u} \tau \sum_{l=1}^{L} \beta_{j l}-(1-\gamma)+\tau-U\right]
\end{array}
$$

Assume that $\operatorname{Tr}\left\{\left(\mathbf{E}\left[\hat{H}_{j j_{n}} \hat{H}_{j j_{n}}^{H}\right]\right)\right\}$ presents the covariance of $\hat{H}_{j j_{n}}$ represented as $R$ and substituting by $U=\sqrt{p_{u}} \tau R^{1 / 2}$ and $V=R^{-1 / 2} \tau^{1 / 2} \hat{H}_{j j_{n}}^{H}$, then (3.30) will become:

$$
\begin{array}{r}
L(\tau, \lambda)=\|U-V\|^{2}-\operatorname{Tr}\left\{\tau \hat{H}_{j j_{n}}^{H} R^{-1}\right\}-\sigma^{2} U\left(\tau-\sqrt{p_{u} \tau}\right)+ \\
\lambda\left[-p_{u} \tau \sum_{l=1}^{L} \beta_{j l}-(1-\gamma)+\tau-U\right]
\end{array}
$$

For 3.32 to be minimum, the first term $\|U-V\|^{2}$ has to be zero, then we equate $\mathrm{U}$ and $\mathrm{V}$ to get:

$$
U=V=\sqrt{p_{u}} \tau R^{1 / 2}=R^{-1 / 2} \tau^{1 / 2} \hat{H}_{j j_{n}}^{H}
$$


Then we get:

$$
\sqrt{p_{u} \tau}=\hat{H}_{j j_{n}}^{H}
$$

From equation (3.12), $\hat{H}_{j j_{n}}=H_{j j_{n}}+\sum_{l=1, l \neq j}^{L} H_{j l_{n}}+n_{j_{n}}^{\prime}$. When $H_{j j_{n}}$ gets extremely large or when the number of BS antennas $A_{n} \gg U$, this leads to optimal asymptotic orthogonality solution (channel hardens) [5,137], and even the linear detectors of low complexity achieve a superior performance (Random processes become deterministic for massive arrays). This property simplifies the derivations and improves the performance. The following property will apply:

$$
\lim _{A_{n} \longrightarrow \infty} \frac{H_{j j_{n}} H_{j j_{n}}^{H}}{A_{n}}=I_{u}
$$

The off-diagonal terms of the $H_{j j_{n}} H_{j j_{n}}^{H}$ matrix become increasingly weaker compared to the diagonal terms, as a result the following property will apply:

$$
\operatorname{tr}\left(H_{j j_{n}} H_{j j_{n}}^{H}\right)=A_{n} \cdot U
$$

Similarly, for the interfering cells (3.36) will also apply for $H_{j l_{n}}$ as follows:

$$
\operatorname{tr}\left(H_{j l_{n}} H_{j l_{n}}^{H}\right)=A_{n} \cdot U
$$

Also $n_{j_{n}}^{\prime}$ will be just an addition of noise to $H_{j j_{n}}+\sum_{l=1, l \neq j}^{L} H_{j l_{n}}$. From the previous equations and substituting in (3.12), then the value of $\hat{H}_{j j_{n}}$ will be:

$$
\hat{H}_{j j_{n}}=(L+1) A_{n} U
$$

Substituting by (3.38) into (3.34) then:

$$
\tau_{\text {opt }}=\frac{(L+1) A_{n} U}{p_{u}}
$$

As $A_{n}$ increases, more power is needed to estimate the channel when pilot symbols are transmitted for different antennas. In that case, $p_{u}$ is proportional to $A_{n}$. The scaling goes as $c * A_{n}$, where c is a decreasing function of the MSE (so larger $\mathrm{c}$ when the MSE is low), where $c \in[0,1]$.

$$
\tau_{o p t}=\frac{(L+1) U}{c}
$$

where $L$ is the number of interfering cells. For low MSE, then c $=1$ and $\tau_{\text {opt }}=(L+1) U$ 


\subsection{Performance Analysis}

We plotted the optimum pilot length for low MSE taking the scaling factor as $\mathrm{c}=1$ in Fig. 3.1. We varied the number of interfering cells, where the optimum length is equal to the number of users when there are no interfering cells. In the case of pilot contamination when the same pilot sequence is reused in the neighboring cells, the optimum pilot is proportional with the number of interfering cells.

Also, Fig. 3.2 shows the optimum value in case of no pilot contamination varying the scaling factor. For low MSE $(c=1)$, the optimum pilot length is slightly greater than the number of users and comparable to it. For high MSE $(\mathrm{c}=0.1)$, the optimum pilot length is large. Optimum pilot length is related to the MSE and proportional to it.

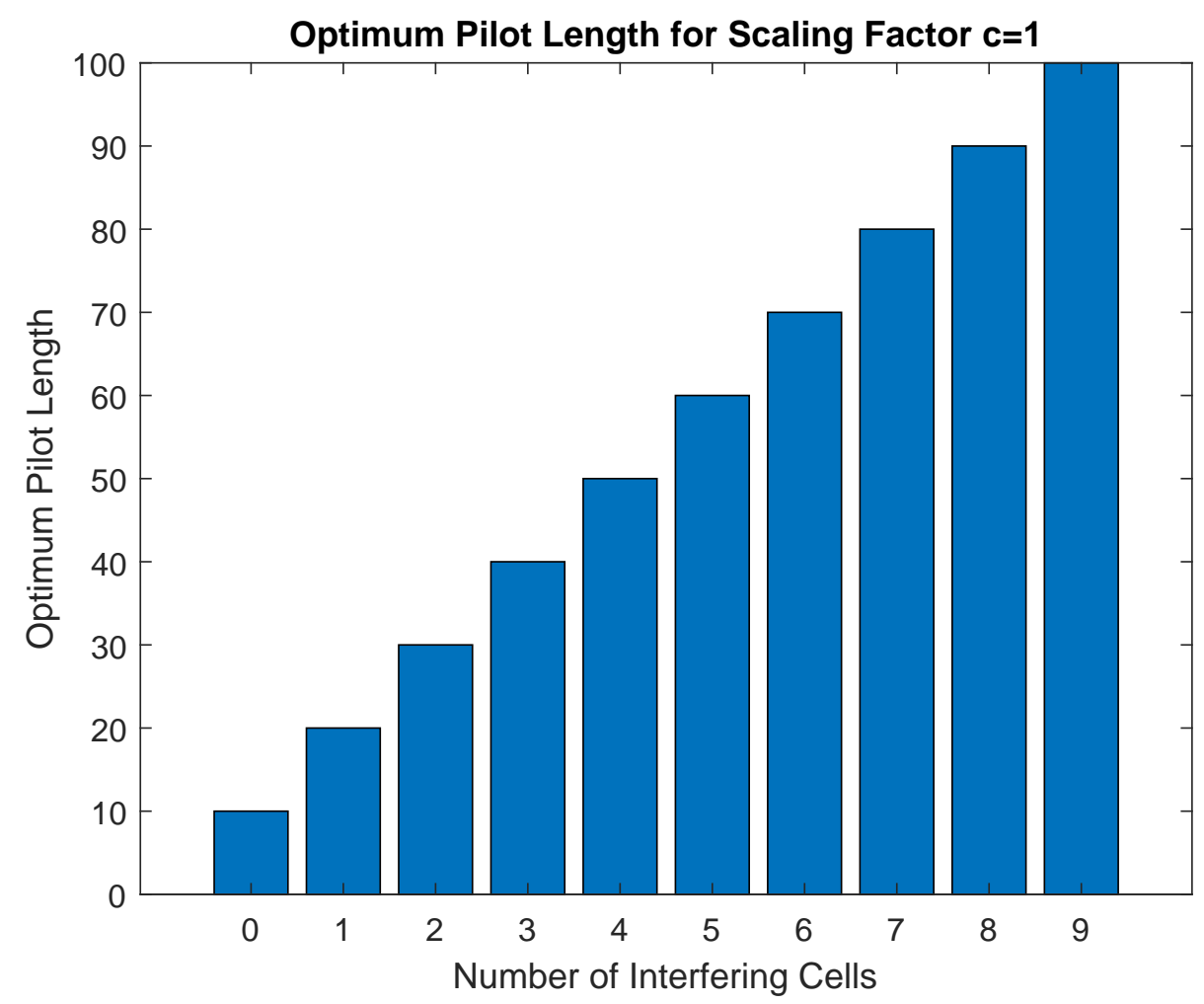

Figure 3.1: Optimum length varying number of interfering cells (L) for $U=10$. 


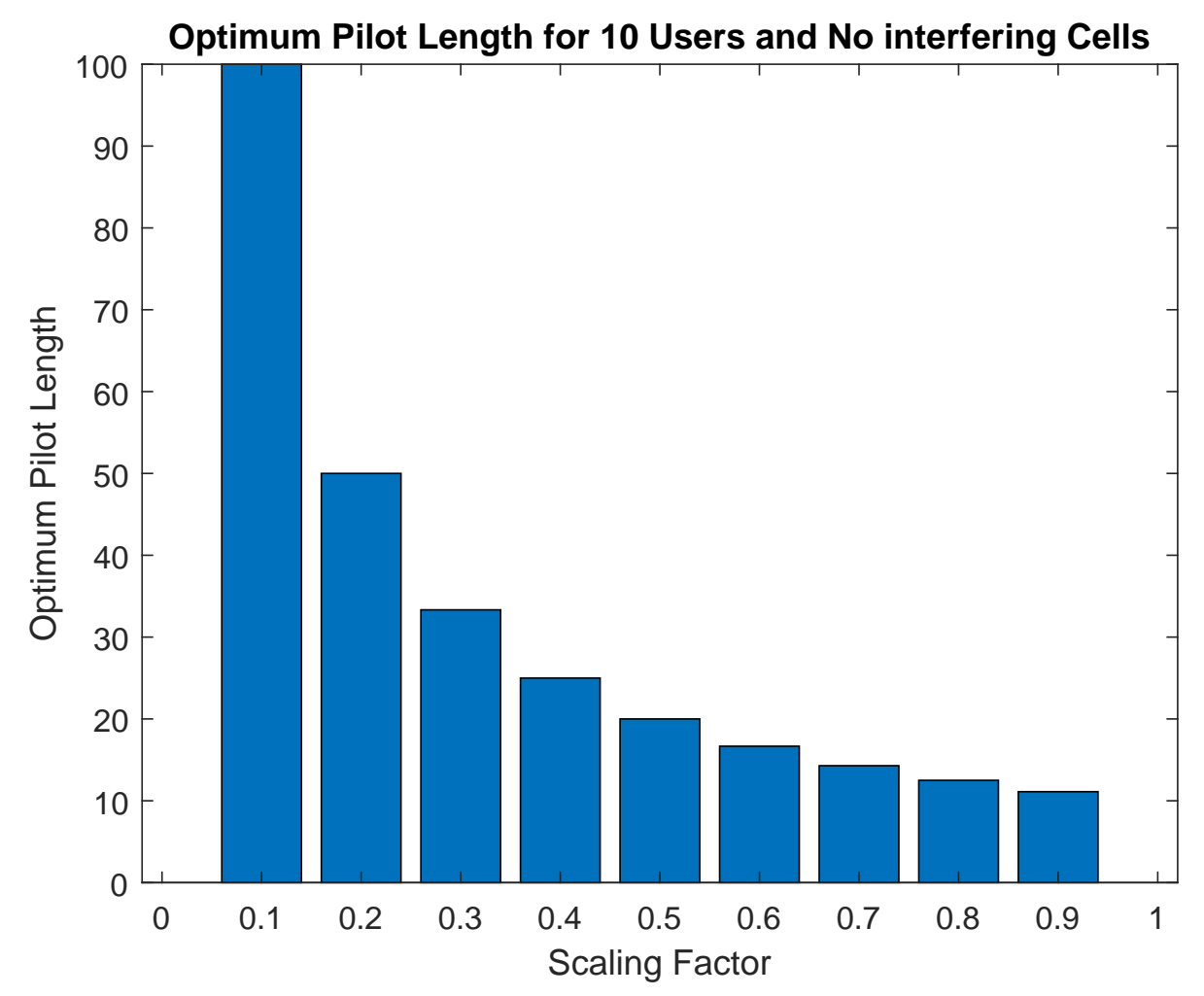

Figure 3.2: Optimum pilot length with no pilot contamination for various scaling factors for $U=10$.

\subsection{Chapter Summary}

In this chapter, we introduced an analytical algorithm to find the optimum pilot sequence length using Lagrange optimization. We applied the least square optimization with MMSE as constraint. Optimum pilot length depends on the number of users, MSE, and number of interfering cells with the same reused pilot sequence. Our results are acceptable as they nearly give similar values with other work in literature. The biggest challenge in using this optimization algorithm is to get an optimum value that is feasible in real wireless networks with some other system dependent constraints. This approach will be very useful if that condition is met. 
CHAPTER 4

\section{Optimization of Micro BSs Positions in HetNets}

\subsection{Background}

In real scenarios, the Macro BSs are usually of a very large number of antennas (massive MIMO), while the Micro BSs have a less number of antennas. This design is enough to achieve very high data rate and speed. Furthermore, the introduction of multi-tier architectures where, small cells with low power nodes coexist with Macro cells will further enhance system performance. However, this architecture requires networks that flexibly connect BSs that operate with different power levels, access methods, and data rates among different tiers [138]. The new system modeling is moving rapidly towards multi-dimensional concept with multiple layers working together [139] to improve the overall sum-throughput gain and to reduce the NMSE. Instead of growing the network horizontally in one dimension with complex designs, the network is growing vertically to reduce signaling overhead, increase energy efficiency, and improve data rate [140]. The old-fashioned cellular network modeling with fixed hexagonal shape cells doesn't meet the modern wireless scenario. Upcoming radio cells are no longer homogeneous and they are of varying sizes and structures depending on the type of BS antennas used and their different capabilities. The main issue with such ultra dense small cells HetNets is the high cumulative interference level which grows rapidly with the increase in the number of nodes and the decrease in the distances between them [141]. Fig. 4.1 shows the cumulative interference power level in ultra dense networks as the number of nodes increases. Also, as the radius of the cell is smaller, the BSs are much closer to each other and this leads to more 


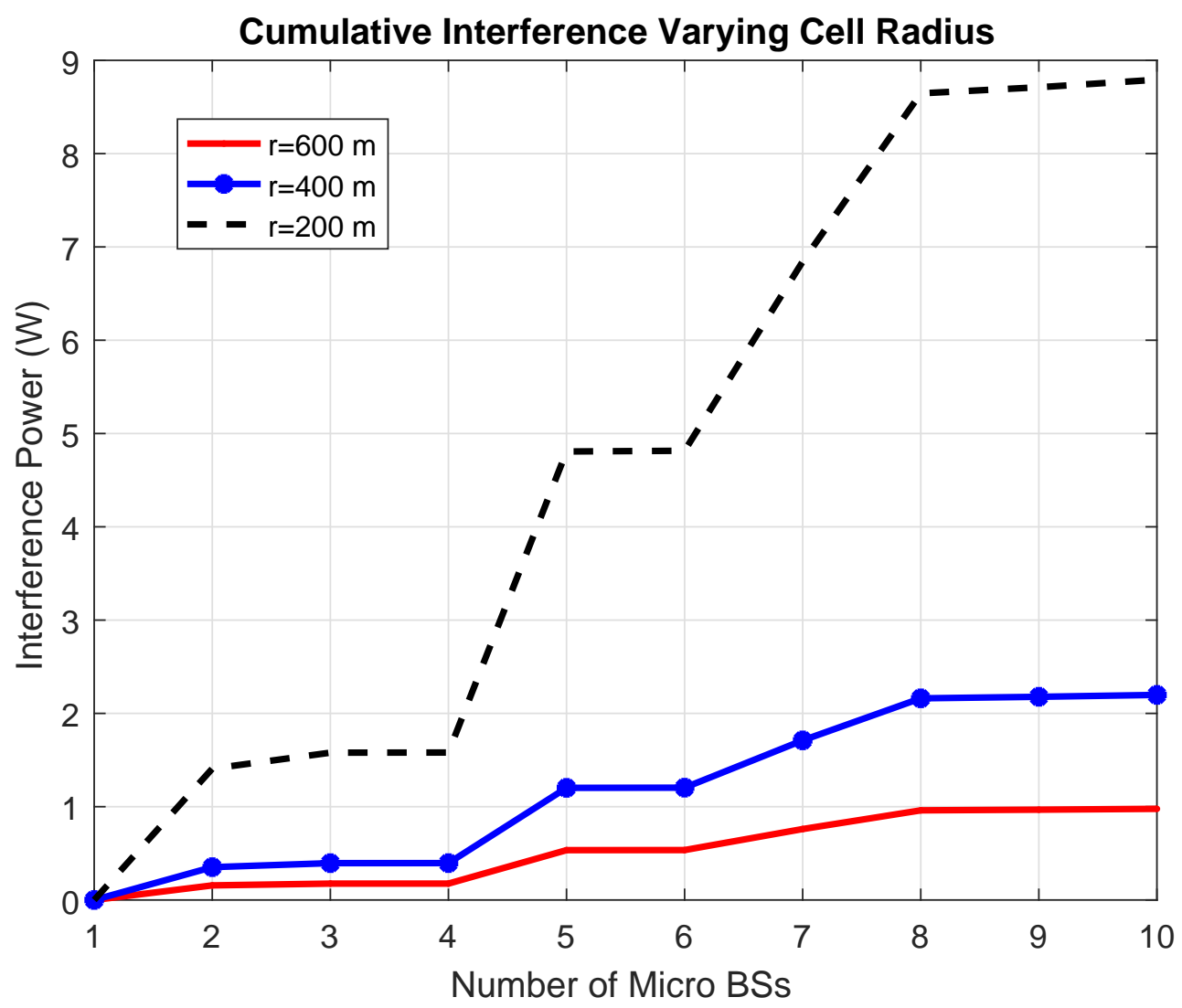

Figure 4.1: Interference power in ultra dense HetNets with ten Micro BSs

interference. Interference is calculated from adjacent Base stations based on large and small scale fading then cumulative interference sum is calculated. Small cell BSs as Micro of Femto BSs can be easily moved and redeployed, which is thought to improve the overall system performance. The main issue in Micro/Femto tier networks is the high interference level especially when those nodes are closely packed. Finding optimum locations for such BSs is a multi variable optimization problem. We optimize the small cell BS locations as they can be easily deployed minimizing the interference to neighboring nodes, which is a crucial problem especially in such dense structures.

\subsection{Related Work}

Authors focused on Micro BSs deployment in ultra dense HetNets. In [142-145], authors addressed optimum small cell BSs deployment to optimize energy efficiency, energy consumption, and area spectral efficiency by choosing the best sets from candidate locations. However, [142,143] did not 
consider the effect of interference as more Micro BSs are added. Also, in [142] Micro BSs tend to be placed in the boundaries of the cell and no BSs are uniformly distributed inside the cell area, which is not practical or realistic. In Table 4.1, we highlight the recent approaches on PPP cellular

Table 4.1: Previous work done on PPP-based cellular networks

\begin{tabular}{|c|c|c|c|c|c|c|}
\hline Reference & e Tiers & Downlink/Uplink & SISO/MIMO & $\begin{array}{c}\text { User } \\
\text { Association } \\
\text { Criteria }\end{array}$ & $\begin{array}{c}\text { Performance } \\
\text { Metric }\end{array}$ & $\begin{array}{c}\text { Year } \\
\text { Publishec }\end{array}$ \\
\hline$[146]$ & $\begin{array}{l}\text { Single and } \\
\text { Multiple }\end{array}$ & NA & NA & $\begin{array}{l}\text { Shortest } \\
\text { Distance }\end{array}$ & $\begin{array}{l}\text { success probability } \\
\text { and energy } \\
\text { efficiency }\end{array}$ & 2013 \\
\hline$[147]$ & Multiple & Downlink & MIMO & NA & $\begin{array}{l}\text { Coverage } \\
\text { probability }\end{array}$ & 2014 \\
\hline$[148]$ & Two-tier & Downlink & MIMO & $\begin{array}{l}\text { Shortest } \\
\text { Distance }\end{array}$ & $\begin{array}{c}\text { Coverage } \\
\text { Probability, } \\
\text { Energy Efficiency, } \\
\text { Throughput }\end{array}$ & 2015 \\
\hline$[149]$ & Multiple & Downlink & MIMO & $\begin{array}{l}\text { Shortest } \\
\text { Distance }\end{array}$ & $\begin{array}{c}\text { Coverage } \\
\text { Probability, Area } \\
\text { Spectral Efficiency }\end{array}$ & 2016 \\
\hline$[144]$ & Multiple & Downlink & NA & $\begin{array}{l}\text { Largest } \\
\text { Mean } \\
\text { Received } \\
\text { Signal } \\
\text { Power }\end{array}$ & Energy Efficiency & 2016 \\
\hline $\begin{array}{l}\text { My } \\
\text { Current } \\
\text { Paper }\end{array}$ & Multiple & Downlink & Massive MIMO & $\begin{array}{c}\text { Load } \\
\text { Distribution } \\
\text { Standard } \\
\text { Deviation/Lo } \\
\text { Balancing }\end{array}$ & Energy Efficiency & 2018 \\
\hline
\end{tabular}

networks and we will start from where [150] has ended.

In [151], the authors studied the Femto BSs deployment problem and suggested a solution to address the high interference challenge which impacts the performance. The authors matched the users to their corresponding Femto access points, then consequently matched the Femto access points to their corresponding service providers. The proposed algorithm maximized the uplink user satisfaction with less complexity. The same problem was addressed by [151], where they studied the small cell deployment challenge, but in disaster scenarios and in emergency situations to confirm 
the safety of everybody. The authors' objective function was to minimize the density of Macro cell BSs maintaining an acceptable level of coverage probability in all tiers. The optimization of low power nodes was addressed in [152] as well. The authors increase the system utility by minimizing the installation cost and maximizing throughput by defining different inter-cell interference cases for the uplink and downlink of Long Term Evolution (LTE) HetNets. Also, the authors set a definition for the interference probability of its set of Pico BSs and Pico users in the uplink and downlink transmissions with respect to the neighboring Macro BSs. They verified through simulation that the proposed algorithm reduces Pico-cell installation cost and improves utility. Authors in [143] proposed a greedy energy efficient deployment method for the Micro BSs in HetNets by choosing an optimum set of locations and then choosing the optimum number of BSs for this problem to maximize energy efficiency. The analytical analysis and simulation results were compared. In addition, authors in [144] studied the problem of Micro BSs deployment in ultra-dense HetNets and its effect on energy efficiency. They derived formulas for the minimum achievable data rate in each tier, for the minimum achievable throughput of the whole network, and energy efficiency with respect to the BS deployment. A very good contribution was considered by [1], where the authors optimized the positions and cluster sizes for fixed relays or can be considered as similar to cluster heads in our approach. Three scenarios were compared to reduce interference and increase throughput. Also, in [153] the authors discussed the optimum deployment in a Macro/Femto scenario. They focused on the impact of interference on the performance of multi-tier systems and proposed to control some deployment parameters and introduced some interference constraints to maximize overall data rate. Authors in [154] modeled the HetNets in a fixed cell size (allocation of users to BSs is based on fixed distances). Inter-cell and inter-tier interference were considered in this model approximating the interference distribution using a Gamma function. Outage probability and per user capacity (as a function of the distance to the cell center) were studied. Performances of single and multi-tier cases were compared. One limitation of this paper is the assumption of a fixed cell size. In turn, the users are associated with the BSs based on fixed distances, which may not be realistic. In addition, the numerical integrals need to be computed. Authors of [155] studied multi antenna HetNets with zero-forcing precoding. They compared the coverage probability and rate per user for both open access (where users are allowed to access any BS in any tier) and closed access networks (where users are granted access to certain BSs in restricted tiers). The authors 
used a cell association criterion based on the maximum SINR. In addition, the authors compared the performance with various combinations of multiple antenna techniques. The performance when the BS is serving a single user in each resource block (by Single Input Single Output (SISO) or SISO or Single User Beamforming (SU-BF) is compared with MIMO configuration serving multiple blocks (by Space Division Multiple Access (SDMA). However, the approximations need more characterizations. In addition, the numerical integrals need extra computational tools to obtain the results. In [147], the authors introduced the Fractional Frequency Reuse (FFR) technique to manage the cross-tier interference (strict FFR and soft frequency reuse). In addition, the authors derived the coverage probability for open-access and closed-access networks (different association policies) and the average rate for the cell edge users. Finally, the authors compared the performance of different FFR and access cases under the full SDMA and SU-BF. In [148], the energy efficiency of different diversity schemes and antenna configurations using adaptive modulation of a two-tier network is studied to ensure a minimum QoS. Energy is saved while obtaining the same throughput by using Femto-cells with sleeping mode capabilities, where only a few of the available antennas are used. This paper identifies that the diversity schemes that provide the highest throughput is different than the ones that achieve the highest energy efficiency. Finally, in [149], the authors derived general and asymptotic success probability expressions for multi-user HetNets with Zero Forcing (ZF) precoding, using a novel Toeplitz matrix representation. In addition, they showed the effect of the BS density on the success probability and derived an optimal BS density for obtaining the maximum Area Spectral Efficiency (ASE) while guaranteeing a certain link reliability. This paper is straightforward with a simple system model. More sophisticated system models should be investigated. In addition, the advantages of introducing Millimeter Wave (mmWave) frequency operation in HetNets is discussed in [156]. The authors discussed the potentials and challenges of the 5G HetNet wireless networks, which merge mmWave technologies into a massive MIMO approach. First, they discussed the extended requirements for $5 \mathrm{G}$ wireless networks with an enormous number of devices that demand more concealment, data rate, better energy and cost efficiency. Then, they discussed the difficulties including traffic arrangement, radio resource management, mobility management, and low-cost beamforming. In the end, they presented some design and case studies to illustrate how to address some of the challenges in 5G HetNets. Finally, in [157], the authors presented some analytical derivations for the conditional association and conditional coverage 
probabilities in HetNets. Also, they minimized the number and density of small cell BSs to achieve an acceptable coverage probability in a disaster scenario.

Due to the flexibility in Wireless Sensor Networks (WSN), several research efforts were done to optimize node location [158-161] (in single as well as multiple cluster scenarios with or without a BS) in terms of network energy consumption, coverage [162], network lifetime, and capacity [163].

\subsection{Contributions and Organization}

In this chapter, we study two optimization problems to find optimum Micro BSs locations to reduce interference, increase SINR, and increase coverage area.

We organize the remainder of this chapter as follows: In Section 4.4, we demonstrate the interconnection of the intermediate distances between BSs. In Section 4.5, we derive the coverage probability of a user in a multi tier network and total network energy efficiency. Section 4.6 presents the statement and description for the non-linear optimization problem. Sections 4.7 discusses the results and findings, while Section 4.8 concludes the chapter.

\subsection{Intermediate Distances Between Micro BSs}

Consider Fig. 4.2, which demonstrates the inter separation between Micro BSs and the distances from the Macro BS at the center to various Micro BSs.

The number of Macro-to-Micro BS distances will be equal to the number of Micro BSs $C$ in the

entire cell. Also, the number of Micro-to-Micro distances will be equal to $\sum_{c=1}^{C}(C-c)$.

Therefore, the total number of intermediate distances in each cell is equal to:

$$
C+\sum_{c=1}^{C}(C-c) .
$$




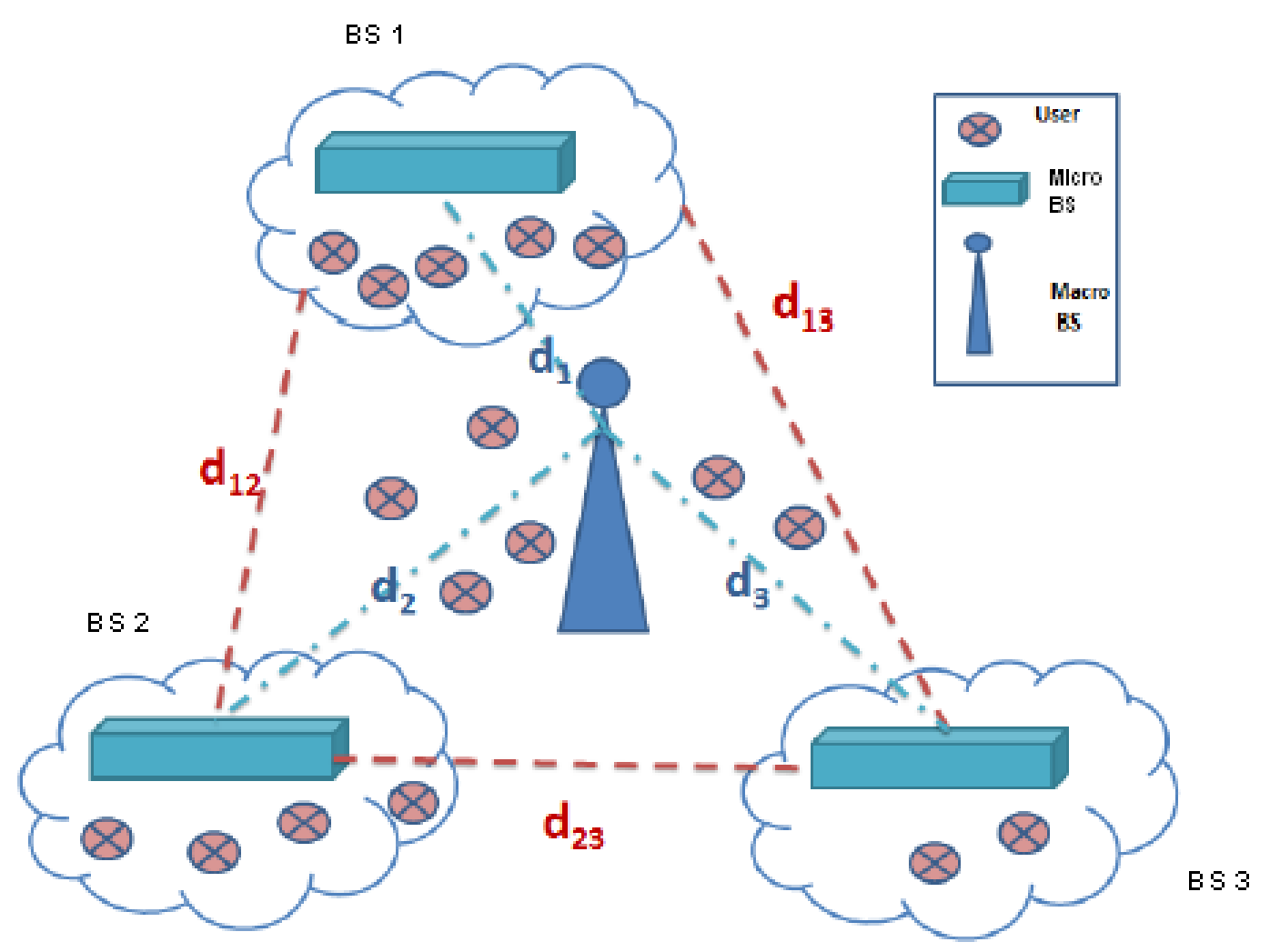

Figure 4.2: Distances between Macro BS and Micro BSs

\subsection{Coverage Probability and Energy Efficiency Analysis in Two-tier Open Access HetNets}

\subsubsection{Coverage Probability Analysis}

SINR for the downlink of a given BS $k$ to its associated UE $u$ on subcarrier $z$ in an OFCDM channel will be:

$$
\gamma_{\mathbf{k}}^{(\mathbf{z})}\left(\mathbf{d}_{\mathbf{k}}\right)=\frac{N^{2} E_{c}^{2} \lambda_{k} P_{T_{k}}\left\|\mathbf{d}_{\mathbf{k}}\right\|^{-\alpha} \sum_{z \in G_{d}}\left(\mathbf{h}_{\mathbf{k}}{ }^{(z)}\right)}{\sum_{q=1, q \neq k}^{k} N^{2} E_{c}^{2} P_{T_{q}}\left\|\mathbf{d}_{\mathbf{q}}\right\|^{-\alpha} \sum_{z \in G_{d}}\left(\mathbf{h}_{\mathbf{q}}{ }^{(z)}\right)+\sigma_{n}^{2}}
$$

where, $N^{2} E_{c}^{2}$ is the power of the $\mathrm{PN}$ sequence used for spreading the signal, $\left\|d_{k}\right\|$ is the distance from the BS to UE $u, \mathbf{h}_{\mathbf{k}}$ is the fading gain from BS $k$ (BS $k$ can either be a Macro or Micro BS) on subcarrier $z, \sigma_{n}^{2}$ is the noise variance, and $\left\|\mathbf{d}_{\mathbf{q}}\right\|$ is the distance from the interfering BSs; $1 \leq q \leq K$. We can define the interference term $\sum_{q=1, q \neq k}^{k} N^{2} E_{c}^{2} P_{q}\left\|\mathbf{d}_{\mathbf{q}}\right\|^{-\alpha} \sum_{z \in G_{d}}\left(\mathbf{h}_{\mathbf{q}}{ }^{(z)}\right)$ as being equal to $\mathbf{I}_{\mathbf{d}_{\mathbf{k}}}$. 
Considering $\mathbb{E}(\cdot)$ to be the expected value, then the expected data rate per UE for BS $k$ is calculated from the above SINR as:

$$
\Re_{\mathbf{k}}^{(\mathbf{z})}=\mathbb{E}\left[\frac{W_{k}}{L_{k}} \log _{2}\left(1+\frac{\left(A_{n}-U+1\right)}{U} \gamma_{\mathbf{k}}^{(\mathbf{z})}\right)\right]
$$

where $W_{k}$ is the total bandwidth assigned to each BS, and $L_{k}$ is the BS load. As $L_{k}$ and $\gamma_{k}$ are independent, then we can write (4.3) as:

$$
\Re_{\mathbf{k}}^{(\mathbf{z})}=\frac{W_{k}}{\mathbb{E}\left[L_{k}\right]}\left(\log _{2}\left(1+\frac{\left(A_{n}-U+1\right)}{U} \gamma_{\mathbf{k}}^{(\mathbf{z})}\right)\right)
$$

where $\mathbb{E}\left[L_{k}\right]$ is the average number of UEs served by a certain tier $k$ BS.

Considering the fact that the UE may or may not be associated to the BS, (4.4) is reduced to:

$$
\Re_{\mathbf{u}}^{(\mathbf{z})}=\mathbf{x}_{\mathbf{k}} \frac{W_{k}}{\mathbb{E}\left[L_{k}\right]} \log _{2}\left(1+\frac{\left(A_{n}-U+1\right)}{U} \gamma_{\mathbf{k}}^{(\mathbf{z})}\right)
$$

where $\mathbf{x}_{\mathbf{k}}$ is a binary variable denoting whether the UE is associated to the BS or not.

If a typical randomly located UE is in coverage, then it connects to a certain BS whose SINR is above its threshold $\zeta_{k}$. Coverage probability in cartesian coordinates is shown as:

$$
P_{c}\left(\gamma_{k}\right)=\mathbb{P}\left(\bigcup_{k \in K} \max _{\mathbf{d}_{\mathbf{k}} \in \phi_{\mathbf{k}}} \gamma_{\mathbf{k}}^{(\mathbf{z})}\left(\mathbf{d}_{\mathbf{k}}\right)>\zeta_{k}\right)
$$

where $\mathbb{P}(\cdot)$ is the probability of the term in brackets. Assuming that we restrict the UE to connect to only one BS at an instance, then:

$$
\begin{aligned}
& P_{c}\left(\gamma_{k}\right)=\mathbb{E}\left[1\left(\bigcup_{k \in K} \max _{\mathbf{d}_{\mathbf{k}} \in \phi_{\mathbf{k}}} \gamma_{\mathbf{k}}^{(\mathbf{z})}\left(\mathbf{d}_{\mathbf{k}}\right)>\zeta_{k}\right)\right] \\
& P_{c}\left(\gamma_{k}\right)=\sum_{k \in K} \mathbb{E} \sum_{\mathbf{d}_{\mathbf{k}} \in \phi_{k}}\left[1\left(\gamma_{\mathbf{k}}^{(\mathbf{z})}\left(\mathbf{d}_{\mathbf{k}}\right)>\zeta_{k}\right)\right]
\end{aligned}
$$

which follows from the union bound.

$$
P_{c}\left(\gamma_{k}\right)=\sum_{k \in K} \lambda_{k} N^{2} E_{c}^{2} \int_{\mathbb{R}^{2}} \mathbb{P}\left(\frac{P_{T_{k}} \mathbf{h}_{\mathbf{k}} \ell\left(\mathbf{d}_{\mathbf{k}}\right)}{\mathbf{I}_{\mathbf{d}_{\mathbf{k}}}+\sigma_{n}^{2}}>\zeta_{k}\right) \mathrm{d}\left(\mathbf{d}_{\mathbf{k}}\right)
$$

which follows from Campbell Mecke Theorem [164], where $\ell\left(\mathbf{d}_{\mathbf{k}, \mathbf{u}}\right)=\left\|\mathbf{d}_{\mathbf{k}, \mathbf{u}}\right\|^{-\alpha}$, and $\mathrm{d}(\cdot)$ is the derivative operator.

$$
P_{c}\left(\gamma_{k}\right)=\sum_{k \in K} \lambda_{k} N^{2} E_{c}^{2} \int_{\mathbb{R}^{2}} \mathbb{P}\left(\left(\mathbf{I}_{\mathbf{d}_{\mathbf{k}}}+\sigma_{n}^{2}\right) \leq \frac{P_{T_{k}} \mathbf{h}_{\mathbf{k}} \ell\left(\mathbf{d}_{\mathbf{k}}\right)}{\zeta_{k}}\right) \mathrm{d}\left(\mathbf{d}_{\mathbf{k}}\right)
$$




$$
P_{c}\left(\gamma_{k}\right)=\sum_{k \in K} \lambda_{k} N^{2} E_{c}^{2} \int_{\mathbb{R}^{2}} \mathcal{L}_{I}\left(\frac{\zeta_{k}}{P_{T_{k}} \ell\left(\mathbf{d}_{\mathbf{k}}\right)}\right) e^{\frac{-\zeta_{k} \sigma_{n}^{2}}{P_{T_{k}}\left(\mathbf{d}_{\mathbf{k}}\right)}} \mathrm{d}\left(\mathbf{d}_{\mathbf{k}}\right)
$$

which arises from the fact that the channel gains are Rayleigh distributed with unity mean, where $\mathcal{L}_{I}(\cdot)$ represents Laplace transform of interference of the term between the brackets. After simplifications, we prove that the coverage probability is obtained as:

$$
P_{c}\left(\gamma_{k}\right)=\left(\frac{\sqrt{\sigma}}{2} N^{2} E_{c}^{2}\right) \sum_{k \in K} \lambda_{k} \times \int_{\mathbb{R}^{2}}\left(e^{\left[\frac{S^{2} \sigma^{2}}{2}-\left(\mu+\sigma^{2}\right) S\right]}\right) \mathrm{d}\left(\mathbf{d}_{\mathbf{k}}\right)
$$

The previous equation can be solved analytically.

Substituting by the value of $S$ assumed at the beginning as $\frac{\zeta_{k}}{P_{T_{k}}^{\ell}\left(\mathbf{d}_{\mathbf{k}}\right)}$, here comes $\alpha$ in (4.12). As a special case for $\alpha=1,(4.12)$ reduces to:

$$
P_{c}\left(\gamma_{k}\right)=\left(\frac{\sqrt{\sigma}}{2} N^{2} E_{c}^{2}\right) \sum_{k \in K} \lambda_{k} \times \frac{\sqrt{\pi} e^{\left(\frac{\delta_{2}^{2}}{4 \delta_{1}}\right)} \operatorname{erf}\left(\frac{2 \delta_{1} \cdot d_{k}+\delta_{2}}{\sqrt{2 \delta_{1}}}\right)}{2 \sqrt{\delta_{1}}}
$$

where $\delta_{1}=\frac{\sigma^{2} \zeta_{k}}{P_{T_{k}}}$, and $\delta_{2}=\frac{\left(2 \mu+\sigma^{2}\right) \zeta_{k}}{P_{T_{k}}}$

The proof is given as follows:

Proof 4.5.1 Let $\frac{\zeta_{k}}{P_{T_{k}} \ell\left(\mathbf{d}_{\mathbf{k}}\right)}=S$, so we want to calculate Laplace transform of interference $\mathcal{L}_{I}(S)$.

$$
\mathcal{L}_{I}(S)=\int_{0}^{\infty} e^{-S \mathbf{d}_{\mathbf{k}}} \mathbf{I}\left(\mathbf{d}_{\mathbf{k}}\right) \mathrm{d}\left(\mathbf{d}_{\mathbf{k}}\right)=\mathbb{E}\left[e^{-S\left(\mathbf{d}_{\mathbf{k}}\right)}\right]
$$

Note that limits of integration are from 0 to $\infty$ as we are integrating over distance which should have a positive value. Authors in [165], proved that interference in HetNets follows a Gaussian distribution, then Laplace transform of interference will follow the same distribution and will be:

$$
\mathcal{L}_{I}(S)=\frac{1}{\sqrt{2 \pi \sigma}} \int_{0}^{\infty} e^{-S \mathbf{d}_{\mathbf{k}}} e^{\frac{-\left(\mathbf{d}_{\mathbf{k}}-\mu\right)^{2}}{2 \sigma^{2}}} \mathrm{~d}\left(\mathbf{d}_{\mathbf{k}}\right)
$$

where $\mu$ and $\sigma^{2}$ are the mean and variance of the Gaussian distribution respectively:

$$
\mathcal{L}_{I}(S)=\frac{1}{\sqrt{2 \pi \sigma}} \int_{0}^{\infty} e^{\frac{-\frac{1}{2}\left(\mathbf{d}_{\mathbf{k}}-\mu\right)^{2}}{\sigma^{2}}-S \mathbf{d}_{\mathbf{k}}} \mathrm{d}\left(\mathbf{d}_{\mathbf{k}}\right)
$$


Let $v=\frac{\mathbf{d}_{\mathbf{k}}-\mu}{\sigma}$, then, $\mathbf{d}_{\mathbf{k}}=\mu+v \sigma$, and $\mathrm{d}\left(\mathbf{d}_{\mathbf{k}}\right)=\sigma \mathrm{d} v$

$$
\mathcal{L}_{I}(S)=\frac{\sqrt{\sigma}}{\sqrt{2 \pi}} \int_{0}^{\infty} e^{-\frac{1}{2} v^{2}-S(\mu+v \sigma)} \mathrm{d} v
$$

After completing the square we get:

$$
\mathcal{L}_{I}(S)=\frac{\sqrt{\sigma}}{\sqrt{2 \pi}} e^{\frac{S^{2} \sigma^{2}}{2}-S \mu} \int_{0}^{\infty} e^{-\frac{1}{2}(v+S \sigma)^{2}} \mathrm{~d} v
$$

Let $u=\frac{1}{\sqrt{2}}(v+S \sigma)$, substituting by the value of $v$, and $\mathrm{d} v=\sqrt{2} \mathrm{~d} u$ we get:

$$
\mathcal{L}_{I}(S)=\sqrt{2} \times \frac{\sqrt{\sigma}}{\sqrt{2 \pi}} e^{\frac{S^{2} \sigma^{2}}{2}-S \mu} \int_{0}^{\infty} e^{-u^{2}} \mathrm{~d} u
$$

The integration $\int_{0}^{\infty} e^{-u^{2}} \mathrm{~d} u$ is evaluated as $\frac{\sqrt{\pi}}{2}$ as shown [166]:

$$
\int_{0}^{\infty} e^{-u^{2}} \mathrm{~d} u=\frac{1}{2} \sqrt{\int_{0}^{\infty} \int_{0}^{\infty} e^{-\left(x^{2}+y^{2}\right)}} \mathrm{d} x \mathrm{~d} y=\frac{1}{2} \sqrt{\int_{0}^{2} \pi \int_{0}^{\infty} r e^{-\left(r^{2}\right)}} \mathrm{d} r \mathrm{~d} \theta=\frac{1}{2}(\sqrt{2 \pi})\left(\sqrt{\frac{1}{2}}\right)=\frac{\sqrt{\pi}}{2}
$$

So we substitute by its value in (4.19) we get:

$$
\mathcal{L}_{I}(S)=\frac{\sqrt{\sigma}}{2} e^{\frac{S^{2} \sigma^{2}}{2}-S \mu}
$$

Assuming that $\sigma^{2}=\sigma_{n}^{2}$, we then substitute by the value obtained from (4.21) into (4.11):

$$
P_{c}\left(\gamma_{k}\right)=\left(\frac{\sqrt{\sigma}}{2} N^{2} E_{c}^{2}\right) \sum_{k \in K} \lambda_{k} \times \int_{\mathbb{R}^{2}}\left(e^{\left[\frac{S^{2} \sigma^{2}}{2}-\left(\mu+\sigma^{2}\right) S\right]}\right) \mathrm{d}\left(\mathbf{d}_{\mathbf{k}}\right)
$$

The previous equation can be solved analytically.

Substituting by the value of $S$ assumed at the beginning to be equal to $\frac{\zeta_{k}}{P_{T_{k}}^{\ell}\left(\mathbf{d}_{\mathbf{k}}\right)}$, where $\ell\left(d_{k}\right)=d_{k}^{-\alpha}$. Here comes $\alpha$ in (4.22).

$$
\begin{gathered}
P_{c}\left(\gamma_{k}\right)=\left(\frac{\sqrt{\sigma}}{2} N^{2} E_{c}^{2}\right) \sum_{k \in K} \lambda_{k} \times \int_{\mathbb{R}^{2}} e^{\left[\frac{\zeta_{k}^{2}}{\left.P_{T_{k}}^{2} \mathbf{d}_{\mathbf{k}}^{-2 \alpha}\right) \sigma^{2}}-\left(\mu+\sigma^{2}\right)\left(\frac{\zeta_{k}}{P_{T_{k}} \mathbf{d}_{\mathbf{k}}^{-\alpha}}\right)\right]} \mathrm{d}\left(\mathbf{d}_{\mathbf{k}}\right) \\
P_{c}\left(\gamma_{k}\right)=\left(\frac{\sqrt{\sigma}}{2} N^{2} E_{c}^{2}\right) \sum_{k \in K} \lambda_{k} \times \int_{\mathbb{R}^{2}} e^{\left[\left(\frac{\zeta_{k}^{2} \mathbf{d}_{\mathbf{k}}^{2 \alpha}}{2 P_{T_{k}}^{2}}\right) \sigma^{2}-\left(\mu+\sigma^{2}\right)\left(\frac{\zeta_{k} \mathbf{d}_{\mathbf{k}}^{\alpha}}{P_{T_{k}}}\right)\right]} \mathrm{d}\left(\mathbf{d}_{\mathbf{k}}\right)
\end{gathered}
$$




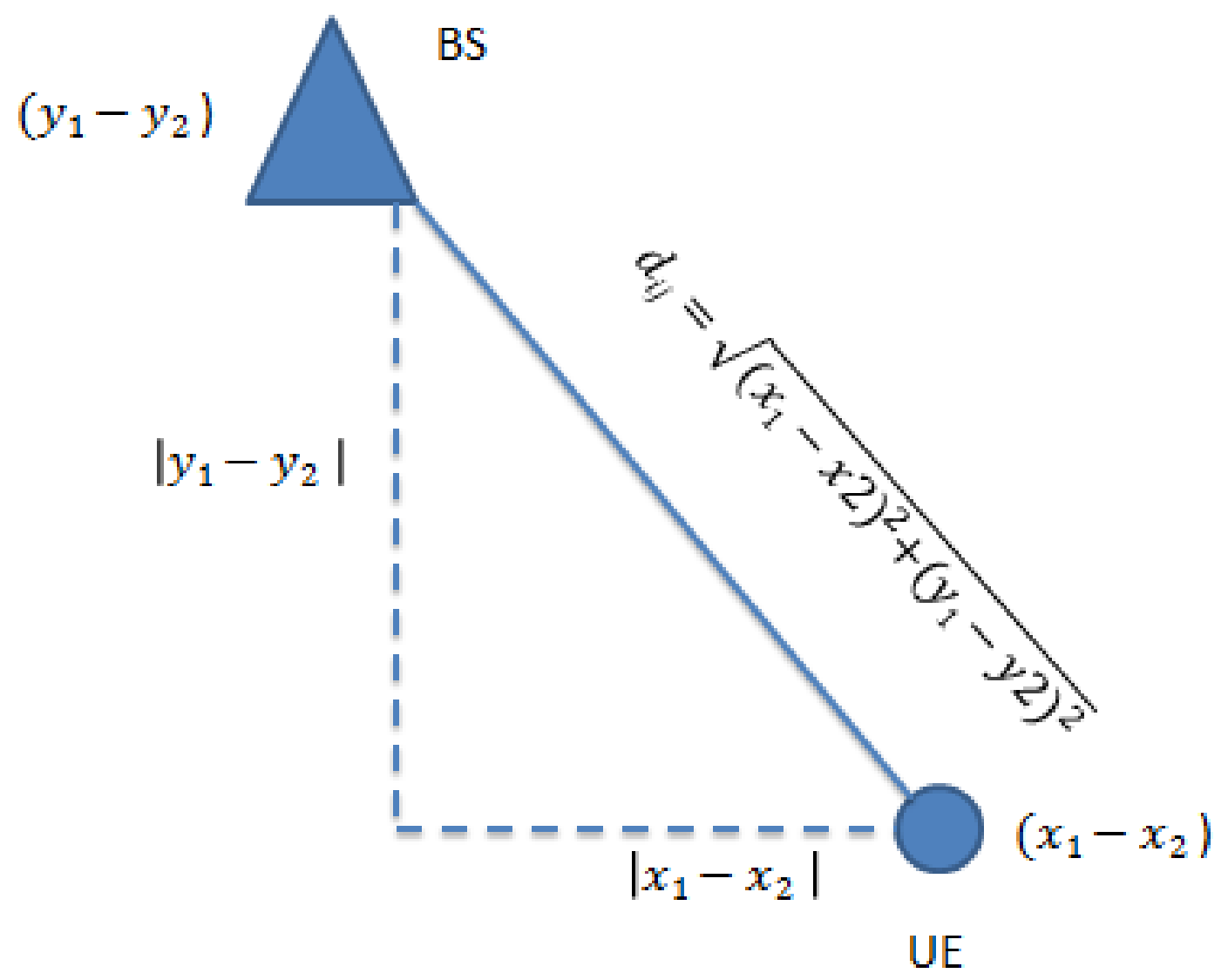

Figure 4.3: Distance between Micro BS and UE

As a special case for $\alpha=1$, (4.24) reduces to:

$$
P_{c}\left(\gamma_{k}\right)=\left(\frac{\sqrt{\sigma}}{2} N^{2} E_{c}^{2}\right) \sum_{k \in K} \lambda_{k} \times \frac{\sqrt{\pi} e\left(\frac{\delta_{2}^{2}}{4 \delta}\right) \operatorname{erf}\left(\frac{2 \delta_{1} d_{k, u}+\delta_{2}}{\sqrt{2 \delta_{1}}}\right)}{2 \sqrt{\delta_{1}}}
$$

where $\delta_{1}=\frac{\sigma^{2} \xi_{k}}{P_{T_{k}}}$, and $\delta_{2}=\frac{\left(2 \mu+\sigma^{2}\right) \xi_{k}}{P_{T_{k}}}$

From Fig. 4.3, for a rectangular cell with center at the origin, of dimensions $2 \cdot a$ and $2 \cdot b$, where the horizontal dimension spans from - a to a along the $x$-axis and the vertical dimension spans from $-b$ to $b$ along the $y$-axis we can derive coverage probability in cartesian $x$ and $y$ coordinates as:

$$
P_{c}\left(\gamma_{k}\right)=\left(\frac{\sqrt{\sigma}}{2} N^{2} E_{c}^{2}\right) \sum_{k \in K} \lambda_{k} \times \int_{-a}^{a} \int_{-b}^{b}\left(e^{\left[\frac{S^{2} \sigma^{2}}{2}-\left(\mu+\sigma^{2}\right) S\right]}\right) \cdot 2 x y\left(x^{2}+y^{2}\right)^{-1 / 2} f_{x}(x) f_{y}(y) \mathrm{d} x \mathrm{~d} y
$$


where $\mathrm{d}\left(\mathbf{d}_{\mathbf{k}}\right)=\mathrm{d}\left(\sqrt{\mathbf{x}^{\mathbf{2}}+\mathbf{y}^{\mathbf{2}}}\right)=\frac{1}{2}\left(x^{2}+y^{2}\right)^{-1 / 2}(2 x \cdot 2 y) \mathrm{d} x \mathrm{~d} y, f_{x}(x)=\frac{1}{2 a}$ and $f_{y}(y)=\frac{1}{2 b}$.

\subsubsection{Energy Efficiency Analysis}

For this section, we derive a new expression for energy efficiency in multi-tier networks. We define $L_{k}$ as the number of UEs associated with a tagged BS in $k^{t h}$ tier, or in other words, it is the traffic load of a BS.

The probability that a tagged UE is connected to a $k^{\text {th }}$ tier BS is [167]:

$$
\mathbb{P}_{k}=\frac{\lambda_{k} P_{T_{k}}^{\frac{2}{\alpha}}}{\sum_{k=1}^{K} \lambda_{k} P_{T_{k}}^{\frac{2}{\alpha}}}
$$

Definition 1 If we define $R_{\text {total }}$ as the minimum achievable throughput of the whole network that depends on coverage probability, expected data rate, and BS density, then its equation is written as:

$$
R_{\text {total }}=\sum_{k=1}^{K} P_{c}\left(\gamma_{k}\right) \lambda_{u} \mathbb{P}_{k} \Re_{k}
$$

Then, energy efficiency is calculated as:

$$
\eta_{E E}=\frac{R_{\text {total }}}{\sum_{k=1}^{K} \lambda_{k} P_{k}}
$$

where $P_{k}$ is the total power consumption of a BS in the $k^{\text {th }}$ tier and is calculated as:

$$
P_{k}=P_{s t_{k}}+\triangle M \mathbb{E}\left[L_{k}\right] P_{T_{k}}
$$

where, $P_{s t_{k}}$ is the static power of the BS in the $k^{t h}$ tier, $\triangle M$ is the load transmission power, and $P_{T_{k}}$ is the transmit power of the $k^{\text {th }}$ tier BS.

After doing assumptions and simplifications, we finally prove that energy efficiency of the network is:

$$
\eta_{E E}=\frac{\left(\begin{array}{c}
\left.\sum_{k=1}^{K} \frac{\lambda_{k}^{3} P_{T_{k}}^{\frac{2}{\alpha}}}{\sum_{k=1}^{K} \lambda_{k} P_{T_{k}}^{\frac{2}{\alpha}}} \log _{2}\left(1+\frac{\left(A_{n}-U+1\right)}{U}\right) \gamma_{\mathbf{k}, \mathbf{u}}\right) \\
\left(\frac{1}{2 \sigma} N^{2} E_{c}^{2}\right) W_{k} \frac{\sqrt{\pi} e^{\left(\frac{\delta_{2}^{2}}{4 \delta_{1}}\right)} \operatorname{erf}\left(\frac{2 \delta_{1} \cdot d_{k}+\delta_{2}}{\sqrt{2 \delta_{1}}}\right)}{2 \sqrt{\delta_{1}}}
\end{array}\right)}{\sum_{k=1}^{K} \lambda_{k}\left(P_{s t_{k}}+\triangle M \mathbb{E}\left[L_{k}\right] P_{T_{k}}\right)}
$$

The details of the proof are presented as follows: 
Proof 4.5.2 We defined $L_{k}$ as the number of users served by a certain tier BS. This number has a distribution, where according to [168], the probability generating function of $L_{k}$ is:

$$
G_{L_{k}}(x)=\mathbb{E}\left[e^{\lambda_{u}(x-1)}\right]=\sum_{L_{k}=0}^{\infty} \frac{\lambda_{u}^{L_{k}} e^{-\lambda_{u} x} x^{L_{k}}}{L_{k} !}
$$

Here Probability Mass Function (PMF) of $L_{k}$ is:

$$
P M F=\mathbb{P}\left(L_{k}=n\right)=\frac{G_{L_{k}^{n}}(0)}{n !}=\int_{0}^{\infty} G_{L_{k}}(x) f(x) \mathrm{d} x
$$

where $f(x)$ is the generalized gamma function or its approximation with shape $q$ and rate $b$, then PMF is.

$$
P M F=\mathbb{P}\left(L_{k}=n\right)=\int_{0}^{\infty} \frac{\left(\lambda_{u} x\right)^{n} e^{-\lambda_{u} x}}{n !} f(x) \mathrm{d} x
$$

We consider the approximated gamma function:

$$
f(x)_{\text {approx }}=\left(C \lambda_{k}\right) x^{q-1} e^{\left(-\lambda_{k} b x^{a}\right)}
$$

where $a=4 \pi R_{c}^{2}$, where $R_{c}$ is half the distance between the Micro BSs after the thinning process. $C=R_{c l} \lambda_{k}$, where $R_{c l}$ is the circle radius of the working area surrounding the Micro BSs.

According to the results obtained from [169], $a=1.0787 \approx 1, b=3.0328 \approx 3, q=3.3095 \approx 3.4$ Assuming $a=1$, then PMF is:

$$
\begin{aligned}
& P M F=\frac{\left(C \lambda_{u}^{n} \lambda_{k}\right)}{n !} \int_{0}^{\infty} x^{n+q-1} e^{-\lambda_{u} x} e^{-\lambda_{k} b x} \mathrm{~d} x \\
& P M F=\frac{\left(C \lambda_{u}^{n} \lambda_{k}\right)}{n !} \int_{0}^{\infty} x^{n+q-1} e^{-\left(\lambda_{u}+\lambda_{k} b\right) x} \mathrm{~d} x
\end{aligned}
$$

Let $u=\left(\lambda_{u}+\lambda_{k} b\right) x$, then $d u=\left(\lambda_{u}+\lambda_{k} b\right) d x, d x=\frac{d u}{\left(\lambda_{u}+\lambda_{k} b\right)}, x=\frac{u}{\left(\lambda_{u}+\lambda_{k} b\right)}$, and $x^{n+q-1}=$ $\left(\frac{u}{\left(\lambda_{u}+\lambda_{k} b\right)}\right)^{n+q-1}$

We substitute by the value of $u$ in (4.37):

$$
\begin{gathered}
P M F=\frac{\left(C \lambda_{u}^{n} \lambda_{k}\right)}{n !\left(\lambda_{u}+\lambda_{k} b\right)\left(\lambda_{u}+\lambda_{k} b\right)^{n+q-1}} \int_{0}^{\infty} u^{n+q-1} e^{-u} d u \\
P M F=\frac{\left(C \lambda_{u}^{n} \lambda_{k}\right)}{n !\left(\lambda_{u}+\lambda_{k} b\right)^{n+q}} \Gamma(n+q)
\end{gathered}
$$


The average number of users served by a tagged BS in $k^{\text {th }}$ tier is:

$$
\begin{gathered}
\mathbb{E}\left[L_{k}\right]=\sum_{n=0}^{\infty} n \cdot P M F \\
\mathbb{E}\left[L_{k}\right]=\sum_{n=0}^{\infty} \frac{C \lambda_{u}^{n} \lambda_{k} n \Gamma(n+q)}{n(n-1) !\left(\lambda_{u}+\lambda_{k} b\right)^{n+q}} \\
\mathbb{E}\left[L_{k}\right]=\sum_{n=0}^{\infty} \frac{C \lambda_{u}^{n} \lambda_{k} \Gamma(n+q)}{\Gamma(n)\left(\lambda_{u}+\lambda_{k} b\right)^{n+q}}
\end{gathered}
$$

For any complex $n$, when $n$ is large:

$$
\Gamma(n)=\sqrt{\frac{2 \pi}{n}}\left(\frac{n}{e}\right)^{n}
$$

Hence the fraction $\frac{\Gamma(n+q)}{\Gamma(n)}$ will become:

$$
\frac{\Gamma(n+q)}{\Gamma(n)}=\left(1+\frac{q}{n}\right)^{n-\frac{1}{2}}\left(\frac{n+q}{e}\right)^{n}
$$

Which is best approximated by 1, so:

$$
\mathbb{E}\left[L_{k}\right]=C \lambda_{k} \sum_{n=0}^{\infty} \frac{\lambda_{u}^{n}}{\left(\lambda_{u}+\lambda_{k} b\right)^{n+q}}
$$

By geometric series test, this function converges, and its value is:

$$
\begin{gathered}
\left|\frac{a_{n+1}}{a_{n}}\right|=\left|\frac{\frac{\lambda_{u}^{n+1}}{\left(\lambda_{u}+\lambda_{k} b\right)^{n+q+1}}}{\frac{\lambda_{u}^{n}}{\left(\lambda_{u}+\lambda_{k} b\right)^{n+q}}}\right| \\
\left|\frac{a_{n+1}}{a_{n}}\right|=\frac{\lambda_{u}}{\lambda_{u}+\lambda_{k} b}
\end{gathered}
$$

Taking its limit to $\infty$ :

$$
\begin{gathered}
\lim _{n \longrightarrow \infty} \frac{\lambda_{u}}{\lambda_{u}+\lambda_{k} b}=\frac{\lambda_{u}}{\lambda_{u}+\lambda_{k} b} \\
\mathbb{E}\left[L_{k}\right]=C \lambda_{k} \frac{\lambda_{u}}{\lambda_{u}+3 \lambda_{k}}
\end{gathered}
$$

As $\lambda_{u} \gg \lambda_{k}$, we can approximate $\mathbb{E}\left[L_{k}\right]$ as $\frac{\lambda_{u}}{\lambda_{k}}$.

From (4.28), we substitute by $\mathbb{E}\left[L_{k}\right]=\frac{\lambda_{u}}{\lambda_{k}}$. Also, we assume that noise variance is equal to interference variance as $\sigma$, we calculate $R_{\text {total }}$ as: 


$$
R_{\text {total }}=\frac{1}{2 \sigma} N^{2} E_{c}^{2} \sum_{k=1}^{K} \frac{\lambda_{k}^{3} P_{T_{k}}^{\frac{2}{\alpha}}}{\sum_{k=1}^{K} \lambda_{k} P_{T_{k}}^{\frac{2}{\alpha}}} W_{k} \log _{2}\left(1+\frac{\left(A_{n}-U+1\right)}{U} \gamma_{\mathbf{k}}\right) \int e^{-\left[S^{2} \sigma^{2}+\left(2 \mu+\sigma^{2}\right) S\right]} \mathrm{d}\left(\mathbf{d}_{\mathbf{k}}\right)
$$

Substituting by (4.50) into (4.31), energy efficiency of the network will be:

$$
\eta_{E E}=\frac{\left(\begin{array}{c}
\left.\sum_{k=1}^{K} \frac{\lambda_{k}^{3} P_{T_{k}}^{\frac{2}{\alpha}}}{\sum_{k=1}^{K} \lambda_{k} P_{T_{k}}^{\frac{2}{\alpha}}} \log _{2}\left(1+\frac{\left(A_{n}-U+1\right)}{U}\right) \gamma_{\mathbf{k}}\right) \\
\left(\frac{1}{2 \sigma} N^{2} E_{c}^{2}\right) W_{k} \int e^{-\left[S^{2} \sigma^{2}+\left(2 \mu+\sigma^{2}\right) S\right]} \mathrm{d}\left(\mathbf{d}_{\mathbf{k}}\right)
\end{array}\right)}{\sum_{k=1}^{K} \lambda_{k} P_{k}}
$$

As a special case for $\alpha=1$ and substituting by the value of $\mathbb{E}\left[L_{k}\right]$ in (4.30), we get:

$$
\eta_{E E}=\frac{\left(\begin{array}{c}
\left.\sum_{k=1}^{K} \frac{\lambda_{k}^{3} P_{T_{k}}^{\frac{2}{\alpha}}}{\sum_{k=1}^{K} \lambda_{k} P_{T_{k}}^{\frac{2}{\alpha}}} \log _{2}\left(1+\frac{\left(A_{n}-U+1\right)}{U}\right) \gamma_{\mathbf{k}}\right) \\
\left(\frac{1}{2 \sigma} N^{2} E_{c}^{2}\right) W_{k} \frac{\sqrt{\pi} e^{\left(\frac{\delta_{2}^{2}}{4 \delta_{1}}\right)} \operatorname{erf}\left(\frac{2 \delta_{1} \cdot d_{k}+\delta_{2}}{\sqrt{2 \delta_{1}}}\right)}{2 \sqrt{\delta_{1}}}
\end{array}\right)}{\sum_{k=1}^{K}\left(P_{s t_{k}}+\triangle M \lambda_{u} P_{T_{k}}\right)}
$$

\subsection{Optimum Locations of Micro BSs.}

BSs in each tier of HetNets are usually randomly deployed according to a PPP. The locations chosen by a PPP may not be the best locations to achieve the best SINR or the least interference. In this chapter, we study the optimum allocation of Micro BSs. We suggest two optimization problems and study performance using extensive simulations. Signal and interference powers are the most important parameters that affect system performance. The goal of any design is to reduce interference, that is why we focused on reducing their bad impact on network performance. Placement of small cell BSs at very close locations zooms out and maximizes the interference. Optimum deployment of BSs directly affects interference.

SINR is defined as the power of a certain signal of interest divided by the sum of the interference power (from all the other interfering signals) and the power of some background noise. When SINR 
is high, BER is low, because for a given modulation a certain SINR at the decoder input must be realized. As the difference between the wanted signal and the unwanted signals (interferers and/or noise) get smaller it will be more difficult to detect the information that is in the wanted signal. BER is proportional to $\frac{1}{\operatorname{SINR}}^{n}$, where $n$ is a subcarrier index or number of bits per symbol.

First, we suggest a new algorithm Optimum Positions for Least Interference (OPLI) to provide the least interference and improve system performance. Next, we suggest an optimization problem for Micro BSs locations to obtain better SINR for the uplink channel from the Micro BSs to the Macro BS.

\subsubsection{Optimum Deployment of Micro BSs (OPLI Algorithm)}

In this subsection, we will study the optimum deployment of Micro BSs to minimize interference.

\subsubsection{Problem Formulation}

Without the loss of generality, we may assume, the Macro BS is at the origin. The Macro cell is assumed to be circular with radius $R$. BSs are initially placed uniformly inside this circular cell. The distances from the Macro BS to any Micro BS $\left(d_{j}\right)$, and that between two Micro BSs $\left(d_{i j}\right)$ are determined respectively as:

$$
\begin{aligned}
& \mathbf{d}_{\mathbf{j}}=\sqrt{\mathbf{x}_{\mathbf{j}}^{2}+\mathbf{y}_{\mathbf{j}}^{2}}=r_{j} \\
& \mathbf{d}_{\mathbf{i j}}=\sqrt{\left(\mathbf{x}_{\mathbf{i}}-\mathbf{x}_{\mathbf{j}}\right)^{2}+\left(\mathbf{y}_{\mathbf{i}}-\mathbf{y}_{\mathbf{j}}\right)^{2}}
\end{aligned}
$$

Remark 4.6.1 We can either consider our optimization problem in cartesian coordinates or in polar coordinates. $x$ and $y$ or $r$ and $\theta$ will be considered as the location variables that we are optimizing as shown in Fig. 4.4.

$\mathbf{I}_{\mathbf{i j}}$ is the interference between two Micro BSs and is equal to $P_{k} \mathbf{h}_{\mathbf{i j}} \mathbf{d}_{\mathbf{i j}}{ }^{-\alpha}$, where $h_{i j}$ is the Rayleigh fading gain. Our algorithm is explained in detail in Algorithm 1 and the flow chart in Fig. 4.5 further clarifies and demonstrates the algorithm.

\subsubsection{Complexity Analysis}

We consider $\mathbf{x}$ and $\mathbf{y}$ positions of every Micro BSs, which are distributed randomly according to the positions obtained from the PPP as shown in Fig. 4.4(note that a Poisson random number is considered for the number 


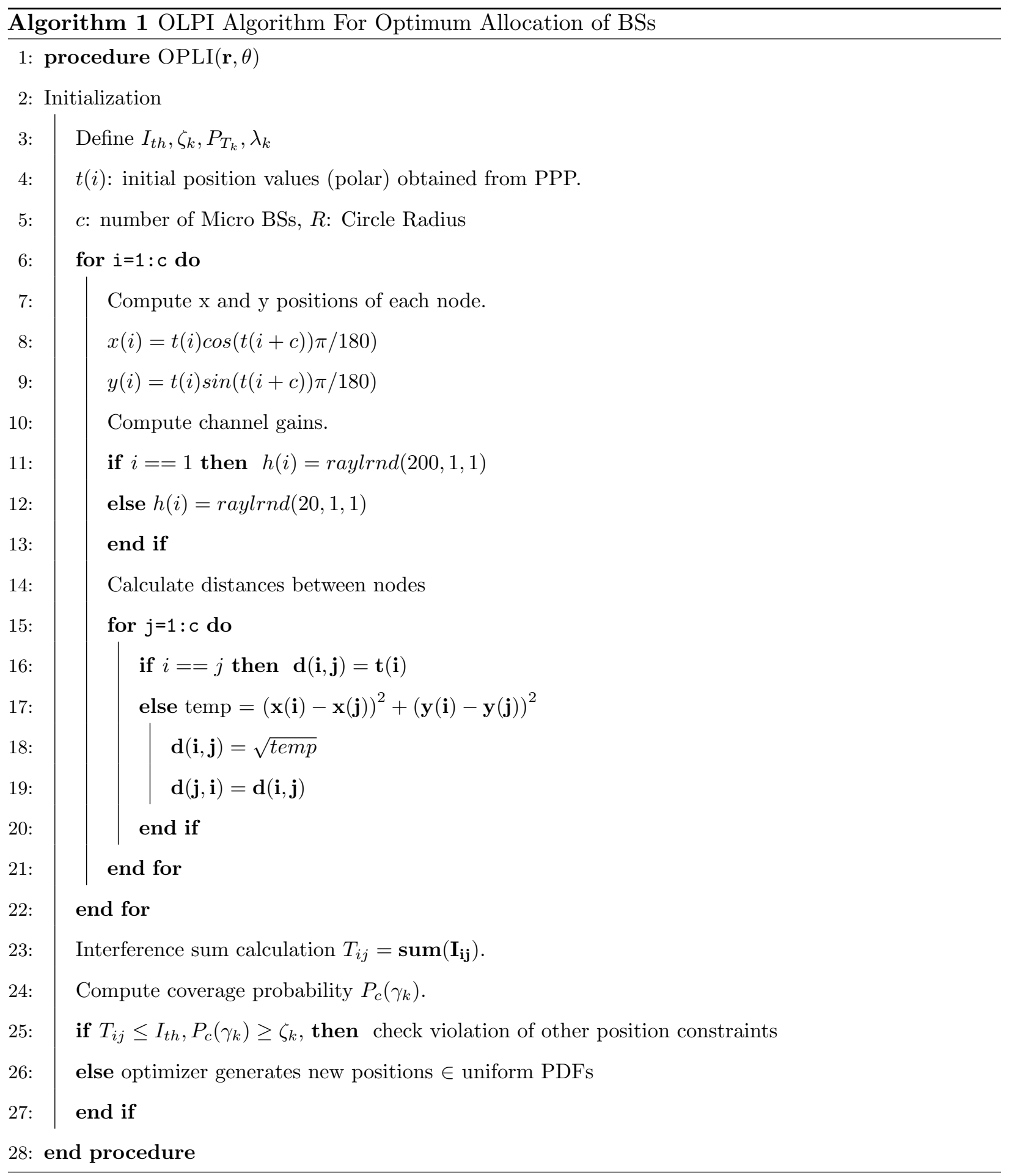




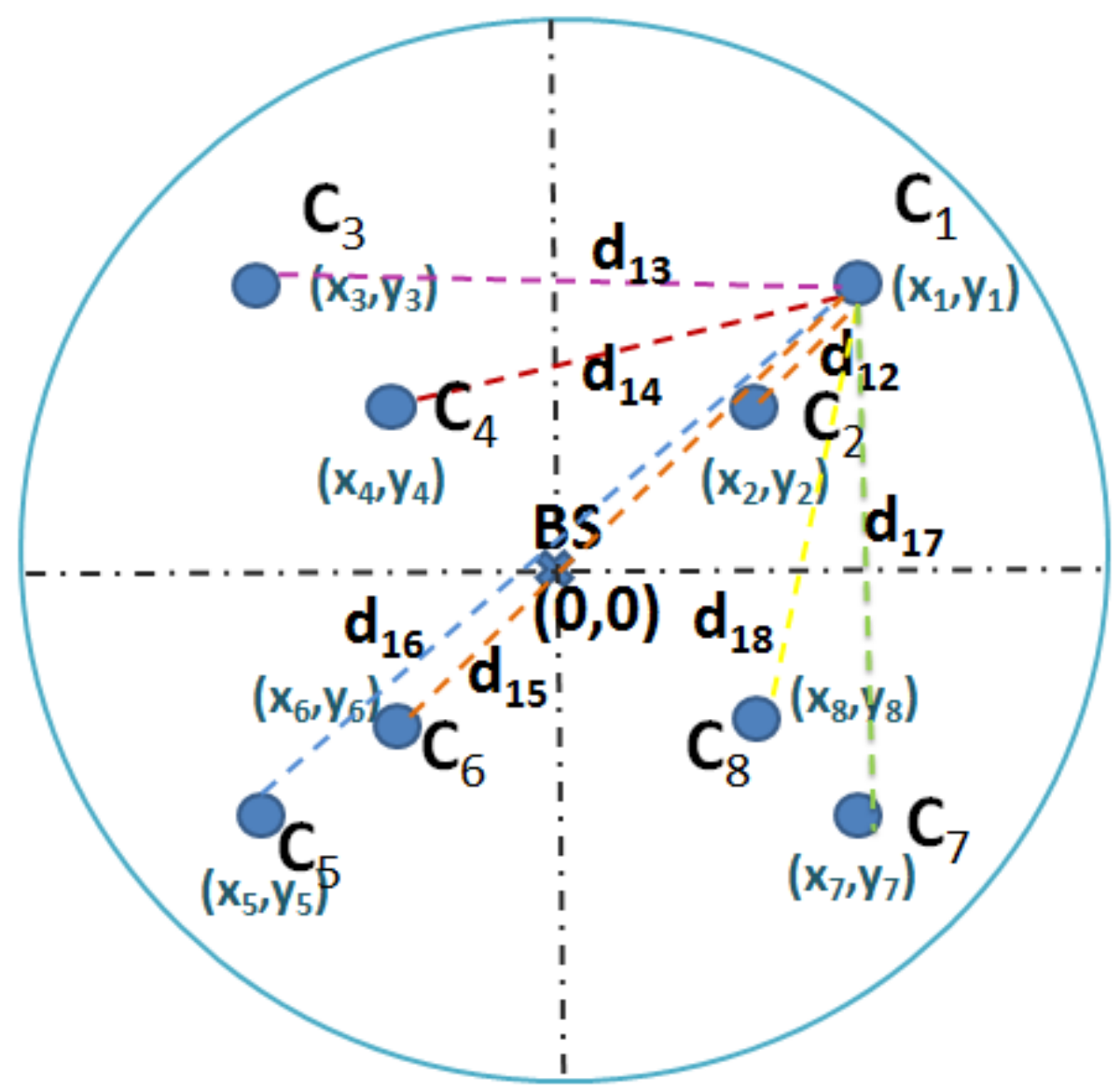

Figure 4.4: Distribution of Micro BSs around the Macro BS.

of BSs and a Uniform distribution is considered for BSs locations). Then, we apply a thinning process based on a certain distance criteria to exclude very near nodes. The $x$ and $y$ positions suggested by the optimizer in every iteration are restricted to be taken from a Uniform Probability Density Function (PDF).

Our optimization problem works by minimizing the sum of total interference $\left(T_{i j}\right)$ from adjacent Micro 


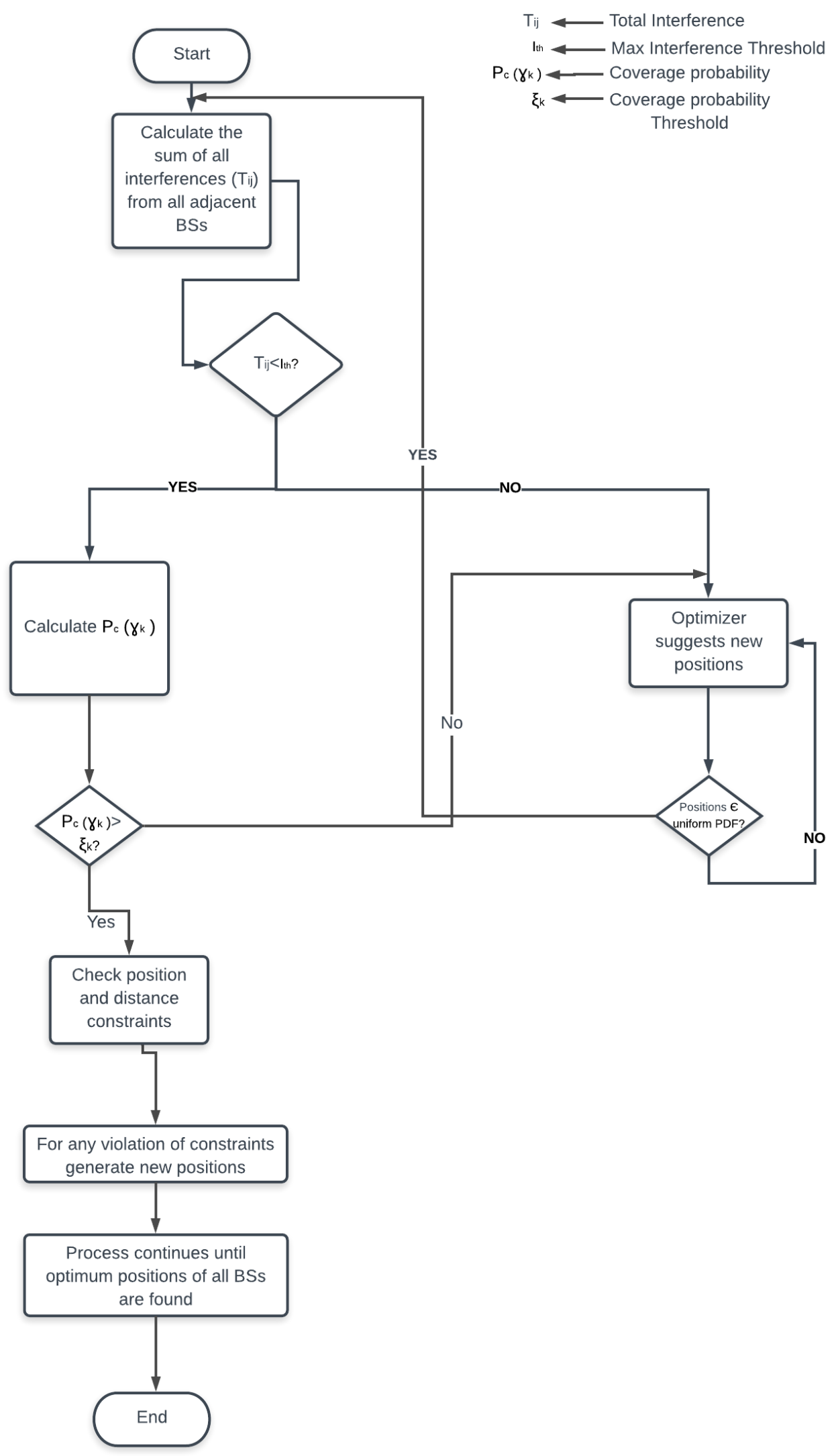

Figure 4.5: Flow chart for our proposed distance optimization algorithm. 
BSs, which can be formulated as:

$$
\begin{array}{ll}
\min _{\mathbf{x}, \mathbf{y}} & \sum_{i=1}^{I} \sum_{j=1, i \neq j}^{J} P_{T_{k}} \mathbf{h}_{\mathbf{i j}}\left(\sqrt{\left(\mathbf{x}_{\mathbf{i}}-\mathbf{x}_{\mathbf{j}}\right)^{2}+\left(\mathbf{y}_{\mathbf{i}}-\mathbf{y}_{\mathbf{j}}\right)^{2}}\right)^{-\alpha} \\
\text { subject to } & C 1: R \geq \mathbf{x}_{\mathbf{j}} \geq-R ; \forall \mathbf{x}_{\mathbf{j}} \in u[-R, R] \\
& C 2: R \geq \mathbf{y}_{\mathbf{j}} \geq-R ; \forall \mathbf{y} \mathbf{j} \in u[-R, R] \\
& C 3: 2 R \geq \mathbf{d}_{\mathbf{i j}} \geq 0 ; \forall \mathbf{d}_{\mathbf{i j}} \in u[0,2 R] \\
& C 4: R \geq \mathbf{d}_{\mathbf{j}} \geq 0 \\
& C 5: \mathbf{T}_{\mathbf{i} \mathbf{j}} \leq I_{t h} \\
& C 6: P_{c}\left(\gamma_{k}\right) \geq \zeta_{k}
\end{array}
$$

where constraint $C_{1}$ and $C_{2}$ depict that the $x$ and $y$ coordinates of the Micro BSs are restricted inside the circular Macro cell area and the positions suggested by the optimizer in every iteration are restricted to be taken from a uniform PDF. Constraint $C_{3}$ restricts that the Micro BSs can lie at maximum at the far edges of the circular working area. Constraint $C_{4}$ defines the position of the Macro BS. Finally, constraint $C_{5}$ and $C_{6}$ restrict that the interference power and coverage probability should be greater than a certain threshold. The variables in this problem are $x$ and $y$ coordinates of every BS, which are optimized. We put restrictions on those $\mathrm{x}$ and y coordinates to lie within the square that goes from $R$ to $R$ and take their values from a uniform probability density function within $[-R, R]$. The distances between every two Micro BSs can take any value from 0 to $2 R$ (if every BS is at the very far end).

If we apply polar coordinates, then $x$ and $y$ coordinates of a given Micro BS would be:

$$
\begin{aligned}
& \mathbf{x}_{\mathbf{j}}=\mathbf{r}_{\mathbf{j}} \cos \left(\theta_{\mathbf{j}}\right) \\
& \mathbf{y}_{\mathbf{j}}=\mathbf{r}_{\mathbf{j}} \sin \left(\theta_{\mathbf{j}}\right)
\end{aligned}
$$

where, $j$ represents a certain Micro BS, $r_{j}$ represents the random variable of the radius of the position of a Micro BS, and $\theta_{j}$ is the angle subtended between the radius and an eastbound line. In polar coordinates, interference sum will remain as it is, but $x$ and $y$ coordinates will be converted to polar coordinates. Now the distance constraints will be in polar coordinates as well and the angle will be from a uniform probability 
density function within $[0,2 \pi]$. The corresponding optimization problem in that case will be:

$$
\begin{array}{ll}
\min _{\mathbf{r}, \theta} & \sum_{i=1}^{I} \sum_{j=1, i \neq j}^{J} P_{T_{k}} \mathbf{h}_{\mathbf{i j}}\left(\sqrt{\left(\mathbf{x}_{\mathbf{i}}-\mathbf{x}_{\mathbf{j}}\right)^{2}+\left(\mathbf{y}_{\mathbf{i}}-\mathbf{y}_{\mathbf{j}}\right)^{2}}\right)^{-\alpha} \\
\text { subject to } & C 1: R \geq \mathbf{r}_{\mathbf{j}} \geq 0 \\
& C 2: 2 \pi \geq \theta_{\mathbf{j}} \geq 0, \forall \theta_{\mathbf{j}} \in u[0,2 \pi] \\
& C 3: 2 R \geq \mathbf{d}_{\mathbf{i j}} \geq 0, \forall \mathbf{d}_{\mathbf{i j}} \in u[0,2 R] \\
& C 4: R \geq \mathbf{d}_{\mathbf{j}} \geq 0 \\
& C 5: \mathbf{T}_{\mathbf{i j}} \leq I_{t h} \\
& C 6: P_{c}\left(\gamma_{k}\right) \geq \zeta_{k}
\end{array}
$$

\subsubsection{Optimum Positions of Micro BSs to Maximize SINR}

For the second optimization problem, our objective is to maximize the SINR of the communication from all Micro BSs to the Macro BS. we consider a maximization problem of SINR in polar coordinates and we considered the previous distance constraints plus SINR constraints. For this, we calculate the SINR matrix for all Micro BSs, assuming BS $j$ as the main (desired) Micro BS communicating with the Macro BS and, $L$ are the interfering BSs.

$$
\operatorname{SINR}_{j_{n}}=\frac{\sum_{n=1}^{N_{t}}\left(d_{j}^{-\alpha}\right)\left|\mathbf{h}_{\mathbf{j}_{\mathbf{n}}}\right|}{\sum_{n=1}^{A} \sum_{l=1, l \neq j}^{L}\left(\left(d_{l}^{-\alpha}\right)\left|\mathbf{h}_{\mathbf{l}_{\mathbf{n}}}\right|+\sigma_{l_{n}}^{2}\right)},
$$

where $j=1: J$

$$
\begin{array}{ll}
\max _{\mathbf{x}, \mathbf{y}} & S I N R_{j_{n}} \\
\text { subject to } & C 1: S I N R_{j_{n}} \geq \gamma_{t h}, \forall j=1: J \\
& C 2: R \geq r_{j} \geq 0, \forall j=1: J \\
& C 3: 2 \pi \geq \theta_{j} \geq 0, \forall j=1: J \\
& C 4: 2 R \geq d_{i j} \geq 0, \forall i=1: I, j=1: J, i \neq j
\end{array}
$$

where constraint $C_{1}$ ensures that SINR for all Micro BSs should be greater than a certain threshold $\gamma_{t h}$. $C_{2}$ ensures that $r_{j}$ the radius coordinate of the location of a Micro BS which is assumed to have a uniform random positive value. $C_{3}$ restricts that $\theta_{j}$ the angle coordinate of the location of a Micro BS inside the circle which, is a uniform random variable that lies in $[0,2 \pi]$. Finally, $C_{4}$ ensures that $d_{i j}$ the distance between any two BSs is assumed to be a uniform random variable lying anywhere in $[0,2 R]$. Note, the farthest location between any two BSs is $2 R$. 


\subsection{Performance Analysis}

Table 4.2 lists the parameters we used in our simulation.

We study optimum Micro BSs deployment. We use interior-reflective Newton optimization method [170172], which is based on a subspace trust region method. This algorithm is used for minimization of general indefinite quadratic functions. In every iteration, the algorithm takes a step towards a point with better function value and approximates the objective function to a simpler one. All optimization problems were solved using interior reflective optimization algorithm with the aid of Matlab optimization tool box using (fmincon) minimization algorithm to solve this nonlinear problem. We considered Matlab optimization toolbox to help us in solving this constrained minimization nonlinear optimization problem. We assume a circular working area. Macro cell is of radius $R=1000 \mathrm{~m}$ where a Macro BS is located at the center at $(0,0)$ location and a number of Micro BSs are uniformly distributed around it. First, we applied a thinning process, where the locations of the Micro BSs were randomly distributed according to a PPP. The BSs which do not satisfy

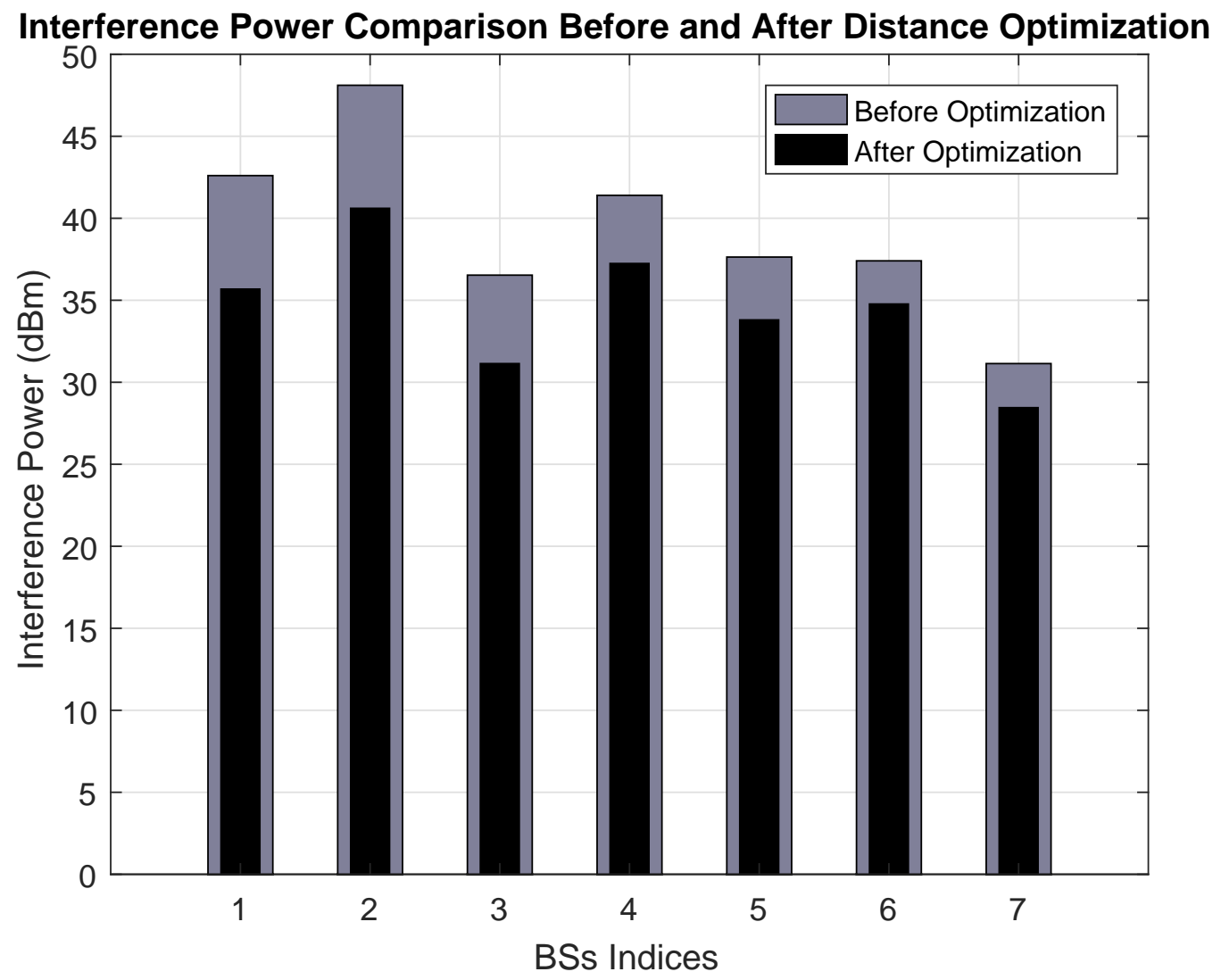

Figure 4.6: Micro-Micro interference comparison (coming from seven adjacent Micro BSs) for the first optimization problem. 
Table 4.2: Parameters used for the suggested

OPLI algorithm

\begin{tabular}{|c|c|}
\hline Parameter & Value \\
\hline Intensity of Initial Micro BSs Positions & 20 \\
\hline Mean Number of UEs of each BS $\left(\lambda_{u}\right)$ & 200 \\
\hline Macro Cell Radius & $1000 \mathrm{~m}$ \\
\hline Min. allowed distance between Micro & $200 \mathrm{~m}$ \\
BSs & 50 Watts \\
\hline Macro BS transmitted power & 20 Watts \\
\hline Noise power & Rayleigh distribution \\
within $[1,200]$
\end{tabular}

a certain distance criteria were excluded. Then, the users which correspond to every BS were generated as well according to a dependant PPP using a parent-child relationship and the thinning process was applied to them. The locations of the generated Micro BSs were obtained, then optimized using our proposed algorithm. The fading gain from the Macro BS to the Micro BSs is chosen from a Rayleigh fading distribution within $[1,200]$, and the fading gain between adjacent Micro BS is chosen from a Rayleigh fading distribution within $[1,20]$.

For results of Section 4.6.1, Fig. 4.6 shows the results of the optimization problem. The interference at all adjacent Micro BSs was reduced by an average of 5 dB. Fig. 4.7 and Fig. 4.8 show a comparison between the signal and interference powers at the locations obtained using the approach in [1] and our proposed optimization techniques, where the yellow circles represent the main signal power and the dark blue and light blue halos represent the interference power. Positions A, B, and C represent the relay station locations (the same as the Micro BSs in our model). Authors of [1] have chosen those three optimum positions empirically. The first position $(750,0)$ is at the cell edge, the second position $(530,530)$ is in the middle of the cells circumference arc and, the third position $(692,286)$ is located at the other cell edge to nullify interference (the distances to the interfering nodes are equal). Fig. 4.7 shows obvious interference represented by the light blue halos while, Fig. 4.8 show no interference between the adjacent nodes.

Fig. 4.9, shows the average energy efficiency of three BSs before and after optimization using (4.31). 
Although energy efficiency is not directly optimized, it improves as an added benefit. Energy efficiency as calculated from (4.32) depends on an error function, which is a function of distance. After optimization the distance between BSs increases, hence error function gives a bigger value and energy efficiency is improved in turn. So energy efficiency is a calculated value that is related to the optimized distance. The curve rises first as $\gamma_{t h}$ increases (because of the data rate), then drops as the coverage probability affects the results. It is clearly seen that energy efficiency improves by optimization. Also, it decreases with the values of $\rho_{1}$ or $\rho_{2}$ (The Macro BS density factor, where $\rho_{k}=\frac{\lambda_{k}}{\lambda_{u}}$ ).

For the second SINR optimization problem, Fig. 4.10 shows a comparison between the signal and interference powers at the locations obtained using the approach in [1] and our proposed optimization algorithm, where the yellow circles represent the main signal power and the dark blue and light blue halos represent the interference power. Positions A,B, and C represent the locations obtained from [1]; whereas, Positions A', B', and C' represent our optimized locations. We can see from Fig. 4.10 that at the first positions $\mathrm{A}, \mathrm{B}$, and $\mathrm{C}$ there was so much interference represented by the dark blue and light blue halos around each Micro BS. After optimization, the locations moved to A', B', and C'. We can see from Fig. 4.10 that each Micro BS is less affected by interference from the neighboring ones and nearly interference is completely eliminated. Fig 4.11, shows a comparison between the power for seven interfering Micro BSs. It is assumed that a single Micro BS is communicating to the Macro BS, while the other Micro BSs are considered as interferers. We found that the SINR for the uplink from the main Micro BS to the Macro BS increased by 2 $\mathrm{dB}$ after applying the optimization. SINR has been maximized for the transmitting BS and minimized for the interfering ones.

\subsection{Chapter Summary}

A good solution to improve network energy efficiency and network capacity is to optimally deploy Micro BSs to reduce interference, which is the main issue especially in dense closely packed nodes. We have seen that although energy efficiency is not directly optimized, it improves as an added benefit due to its dependence on error function. In this chapter, we suggested novel algorithms for optimizing Micro BSs locations to increase received signal power, reduce interference, and improve coverage. Conventional interference mitigation algorithms are spectrum partitioning based, which suffer from some drawbacks (They are costly, spectrum inefficient, and do not meet the increasing data demand of the network in case of ultra dense small cells that

could degrade quality of service and throughput). We suggest novel algorithms that may be considered as location-based interference mitigation algorithms. We minimized sum of interference level and maximized SINR. The proper choice of the constraints contributed in obtaining such feasible results. The results after optimization show a significant improvement. Also, we compared our results with other work and showed 
that our suggested algorithms are promising in boosting the performance and reducing interference. 


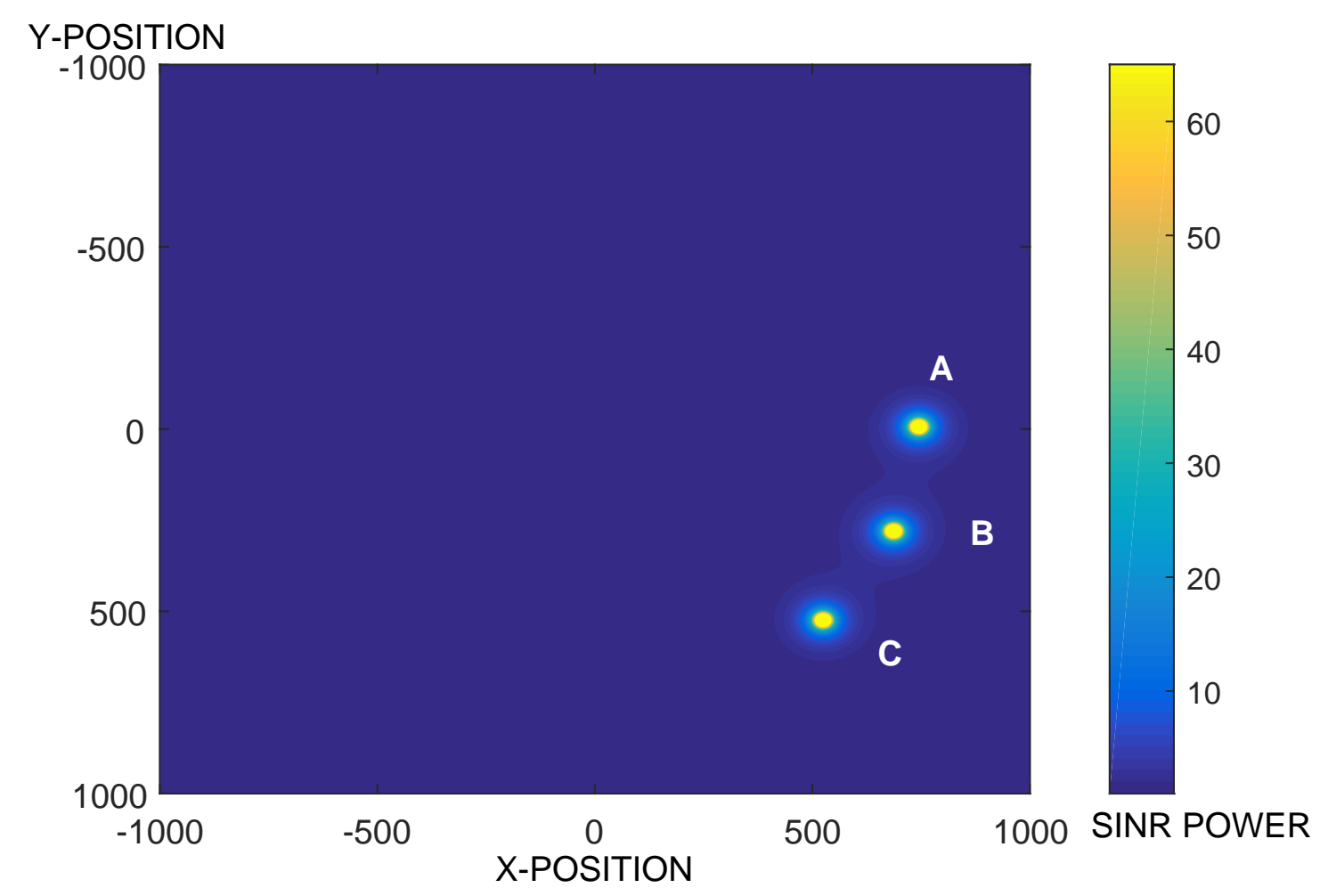

Figure 4.7: Positions of three BSs from [1]

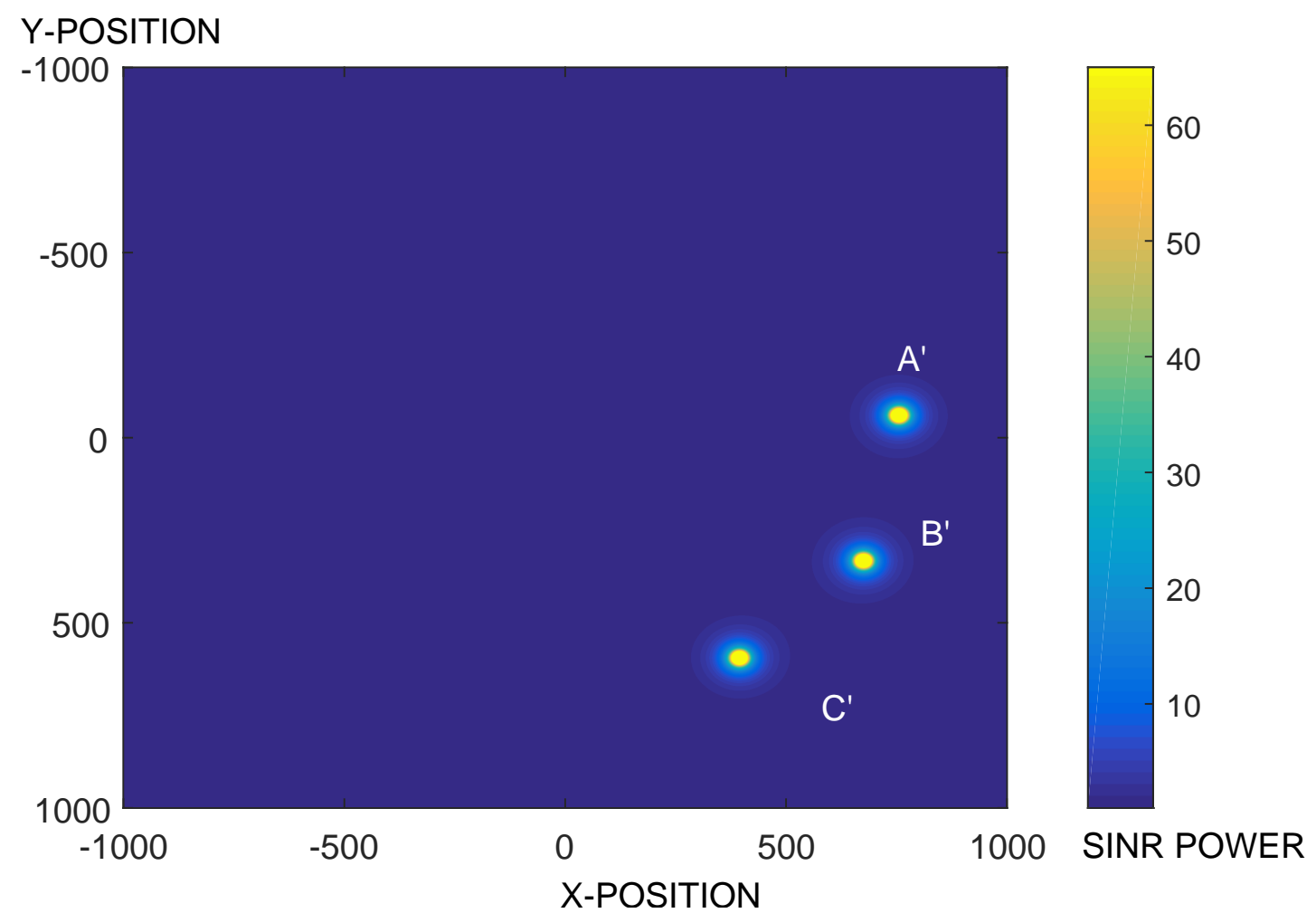

Figure 4.8: Position of three BSs after applying our suggested first optimization algorithm 


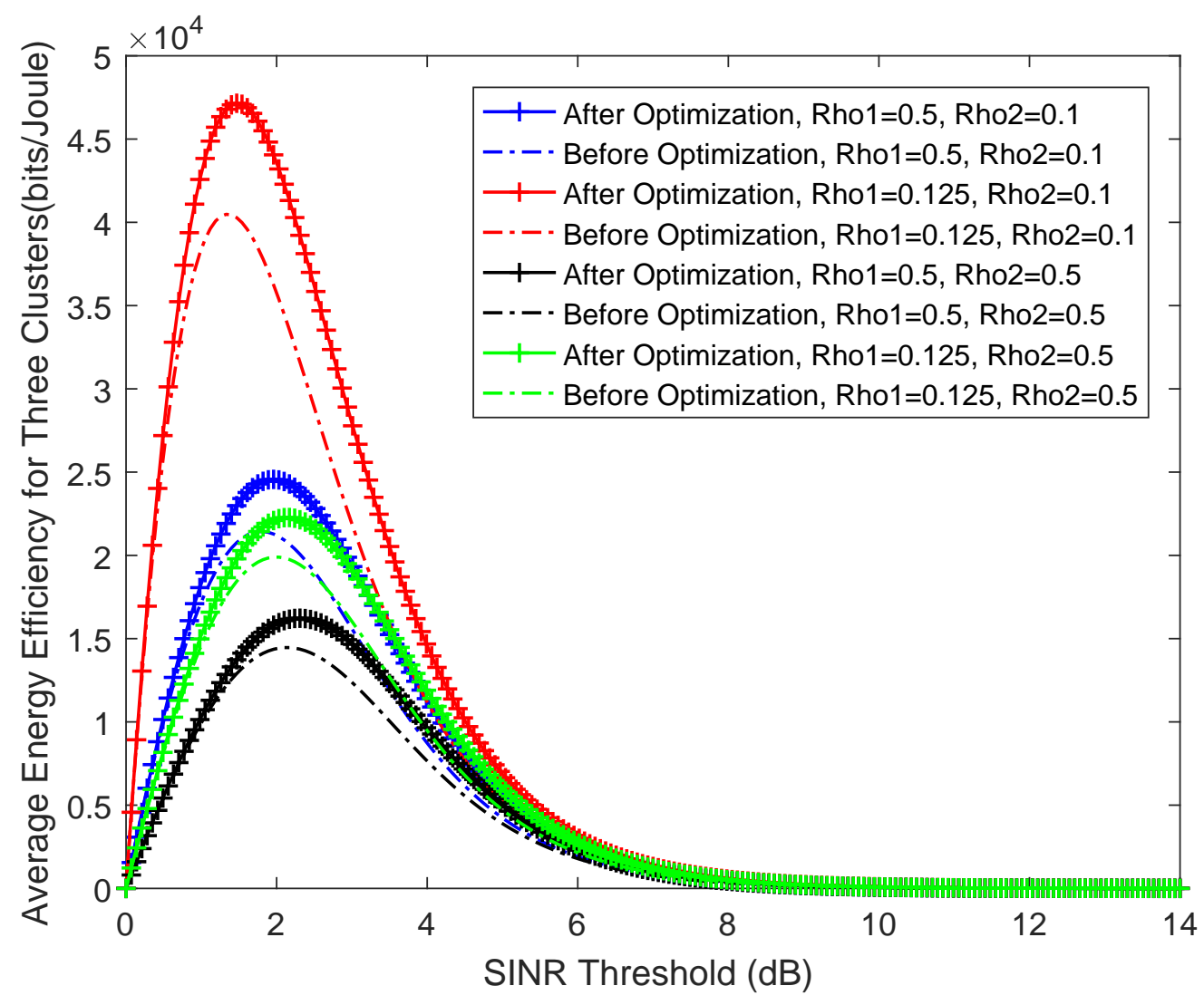

Figure 4.9: Average energy efficiency comparison before and after optimization 
Signal and Interference Power Comparison With Reference Paper

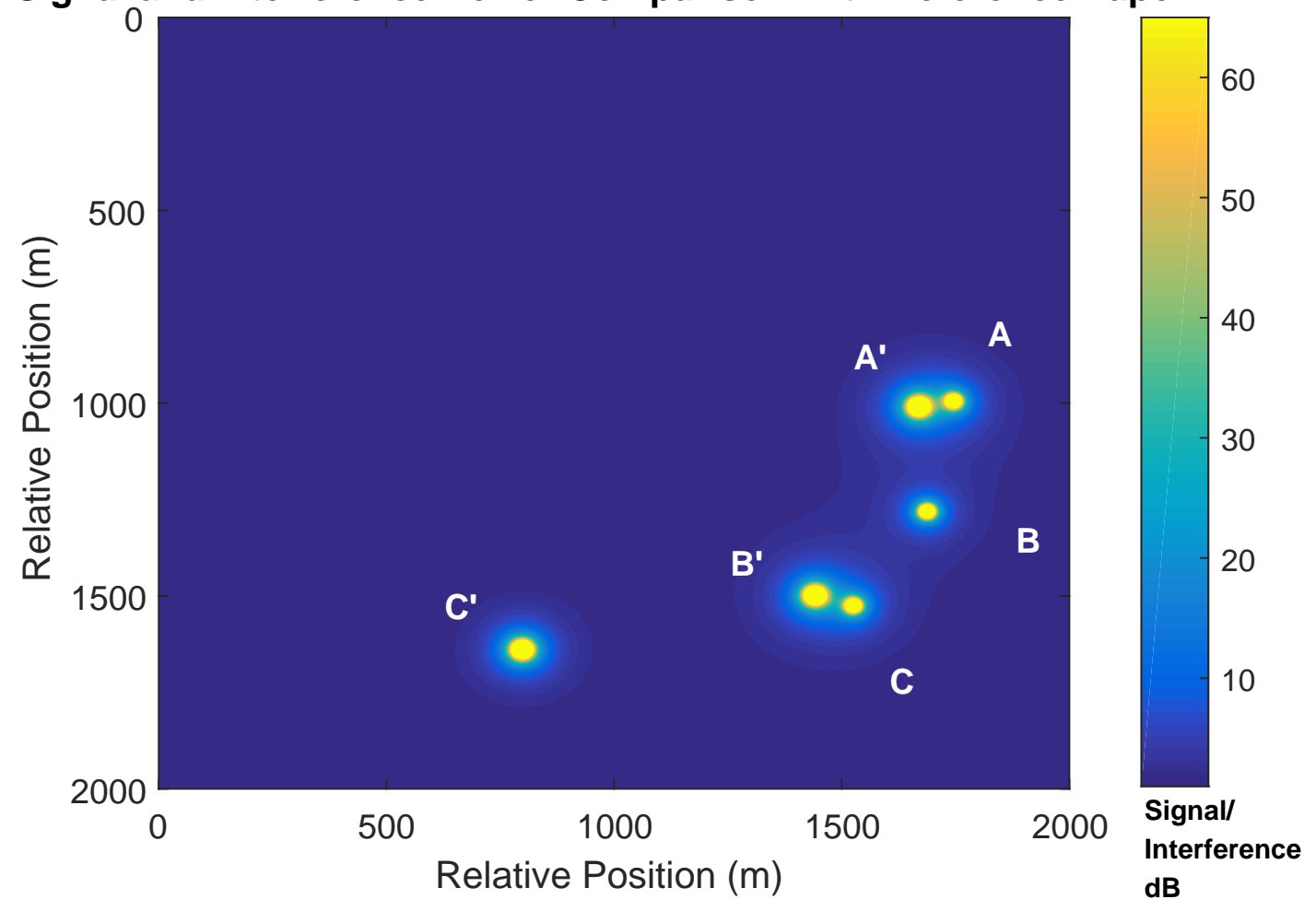

Figure 4.10: Comparison of our work with [1] for the second optimization problem. 


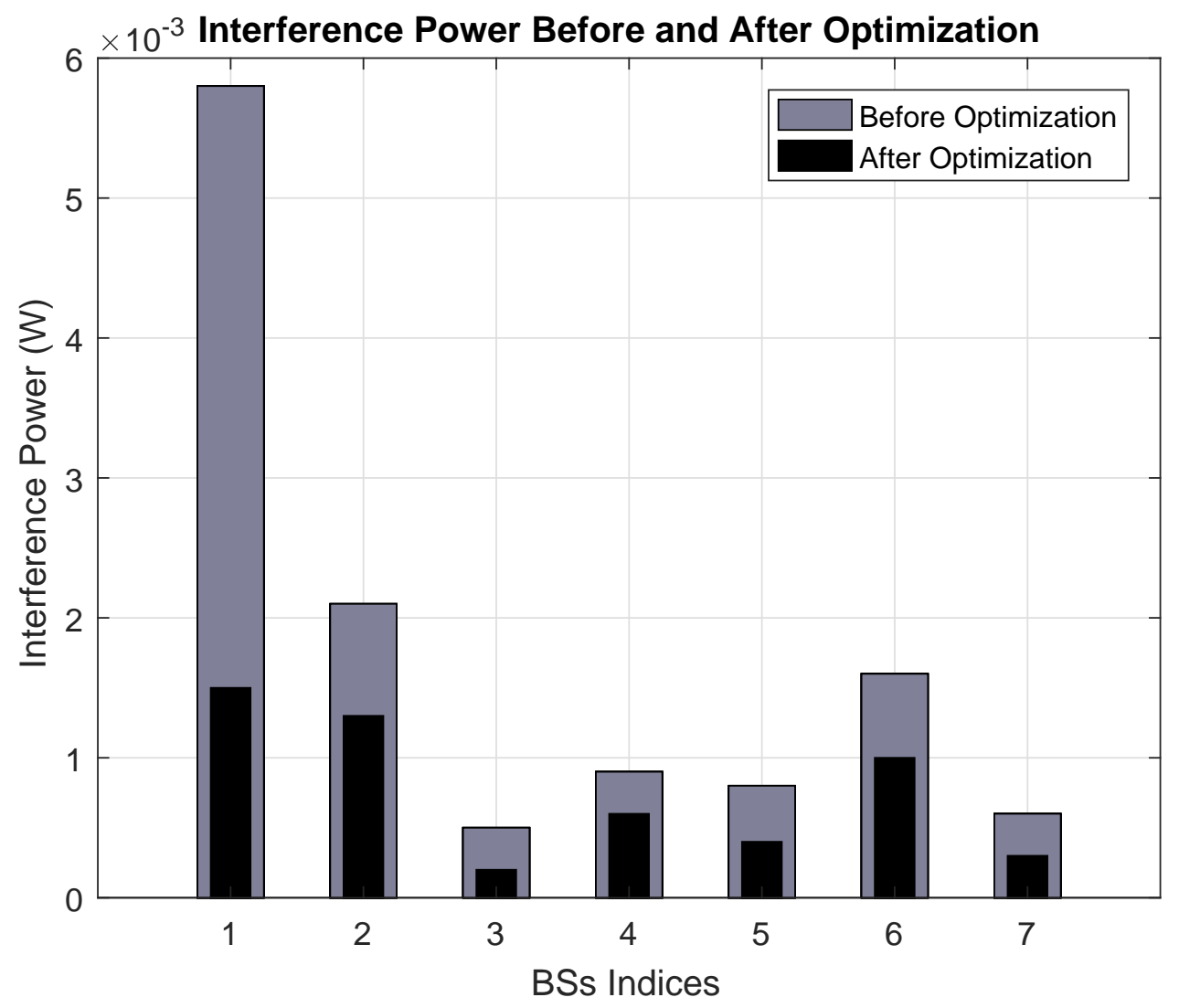

Figure 4.11: Comparison between interference power values for the second optimization problem 
CHAPTER 5

\section{Optimizing Macro BSs Locations in HetNets for}

\section{Eliminating Interference}

\subsection{Introduction}

PPP is thought of as the best stochastic geometry process that would represent the randomness and in-homogeneity of HetNets [154]; However, placing the BSs at the locations obtained from the PPP does not provide the best placement to improve system performance and reduce interference. Furthermore, note that once a BS is installed, it is not easy to move Macro BSs with their huge infrastructure from their first assigned locations. However, effective deployment and design initially before the first installment of BSs can be beneficial. The goal is not to move the base stations that are already installed (despite the possibility of doing that), but the goal is to install new ones in a better way. Regarding moving the old ones, If the installer finds more benefits for moving them, besides studying other parameters as well (like climate, inhabited areas, difficulty etc.) then every case is studied individually.

In this chapter, we propose a novel way to find optimum locations for Macro BSs to achieve the best-expected performance. The coverage area of any cell depends on the transmitted Radio Frequency (RF) power from the BS, shadow and fading margin, minimum signal strength, propagation slope, and deployment distance. The main issue in multi-tier networks is the high interference level especially when those nodes are closely packed [139]. If the interference among users can be minimized, say by optimizing the BSs locations, then the coverage area in turn can be increased and more UEs can be associated with BSs and the quality of service can be boosted. Finding optimum locations for such BSs is a multi-variable optimization problem. In this work, we provide a combined deployment algorithms for both Macro and Micro BSs in HetNets. We 
introduce an efficient algorithm for Macro BSs deployment to help installers choose the best locations that would provide the least interference from neighboring Macro and edge Micro BSs in adjacent cells. After nearly isolating Macro BSs from all sources of interference, we work on selecting the best positions for core Micro BSs deployment to minimize interference from adjacent Micro BSs of the same cell.

\subsection{Related Work}

Several research efforts were directed towards the problem of optimum BSs deployment. In [173], the authors introduced an evolutionary optimization technique for optimizing the location of BSs in large scale HetNets maximizing the system satisfaction function which depends on the BS position coordinates and traffic demand. The proposed method has overcome the performance deterioration with the growth of search space, especially in such large scale systems by grouping the cells together according to their mutual interference. In [173], the authors divided the problem of optimum BS deployment into sub problems to overcome the limitation of evolutionary algorithms when HetNet size is very large. The authors group cells together depending on their mutual interference. In addition, the authors introduced a new evolutionary optimization approach based on the variable-length genetic algorithm. The objective function to maximize system throughput converged rapidly which reflects the good performance that outperformed other methods. In [174], authors studied an optimum BS deployment algorithm for the cooperating and non-cooperating BSs cases using game theory. The SINR was the revenue taking into account the SINR-equilibrium. They determined the locations at which mobile terminals prefer to connect to a given BS. Also, the authors provided analytic expressions for the cell boundaries in the SINR-equilibrium. The authors in [143] propose a simple yet efficient method for optimum deployment and optimum number of BSs in HetNets to improve energy efficiency and network capacity. The introduced method selects the optimum locations from a set of candidate positions and provides better performance working on the weighted sum of energy efficiency gain over all scenarios. Also, $[142,144]$ initially assume an area deployed with Macro BSs only, which is not a realistic and practical scenario. An interesting paper [1] studies the best position to place the fixed Relay Stations (RS) from a set of empirical good solutions in a LTE network by analyzing the RS deployment in three positions within the cell area. The best RS position was set in the middle of the cells circumference arc. Further analysis showed that the network performance is improved with the RS deployment at the cell edge for all considered positions, since this area is characterized by a lower received power with higher interference levels. The optimum RS locations increased the percentage of the area served by an RS and the mean data rate in the cell. The optimum positions were concluded to be in the middle of the cells circumference arc closer to the cell edge. Also, network performance was improved when the number of RSs is increased. The problem of BS location and antenna configuration optimization for cellular networks was studied by [175]. The authors used network 
cost as an objective function to guarantee coverage for each demand point and they put constraints on SINR, area coverage, capacity, and quality of service. The authors considered a discretized map with demand points to illustrate the network mathematical model. The interference is minimized by defining a certain protection distance between the UEs. In order to achieve a higher quality of service, a longer deployment distance should be applied. Also, in [153] the authors discussed the optimum deployment in a Macro/Femto scenario. They focused on the impact of interference on the performance of multi-tier systems and proposed to control some deployment parameters and introduced some interference constraints to maximize overall data rate. Finally, in [157], the authors presented some analytic derivations for the conditional association and conditional coverage probabilities in HetNets. Also, they minimized the number and density of small cell BSs to achieve an acceptable coverage probability in a disaster scenario.

\subsection{Contributions and Organization}

In this chapter, we introduce an efficient algorithm for Macro BSs deployment to help installers and designers choose the best locations that would provide the least interference from neighboring Macro and edge Micro BSs in adjacent cells. We were able to isolate every Macro BS along with its associated Micro BSs and users from all sources of interference coming from neighboring Macro and Micro BSs in adjacent cells. We will treat every Macro cell as an independent unit, canceling the effect of neighboring BSs in adjacent cells. After nearly isolating Macro BSs from all sources of interference, we work on selecting the best positions for core Micro BSs deployment to minimize interference from adjacent Micro BSs of the same cell.

We organize the remainder of this chapter as follows. In Section 5.4, we explain all sources of interference. Section 5.5 presents the description of Dummy Intersecting Circle (DIC) algorithm and the combined Modified Dummy Intersecting Circle (MDIC) algorithm for optimizing Macro and Micro BSs locations. Numerical results are discussed in Section 5.6 and sensitivity analysis for various thresholds values is presented in Section 5.7. Finally, Section 5.8 concludes the chapter.

\subsection{Channel and Interference Models}

We assume an imaginary circle around every Macro BS approximately representing the cell coverage area. Considering the line of sight path between Macro BSs, we may join the line between the centers of the two Macro BSs and take the intersection point of this line with the circle as the location of a dummy Micro BS at the edge. Note that the straight line intersects every circle at two locations, so we take the minimum values as, $\left(X_{u}, Y_{u}\right)$ for the first circle intersects and $\left(X_{v}, Y_{v}\right)$ as the second circle intersects. The Macro and Micro BSs transmission powers are $P_{M}$ and $P_{m}$ respectively. There are many sources of interference in our system. 


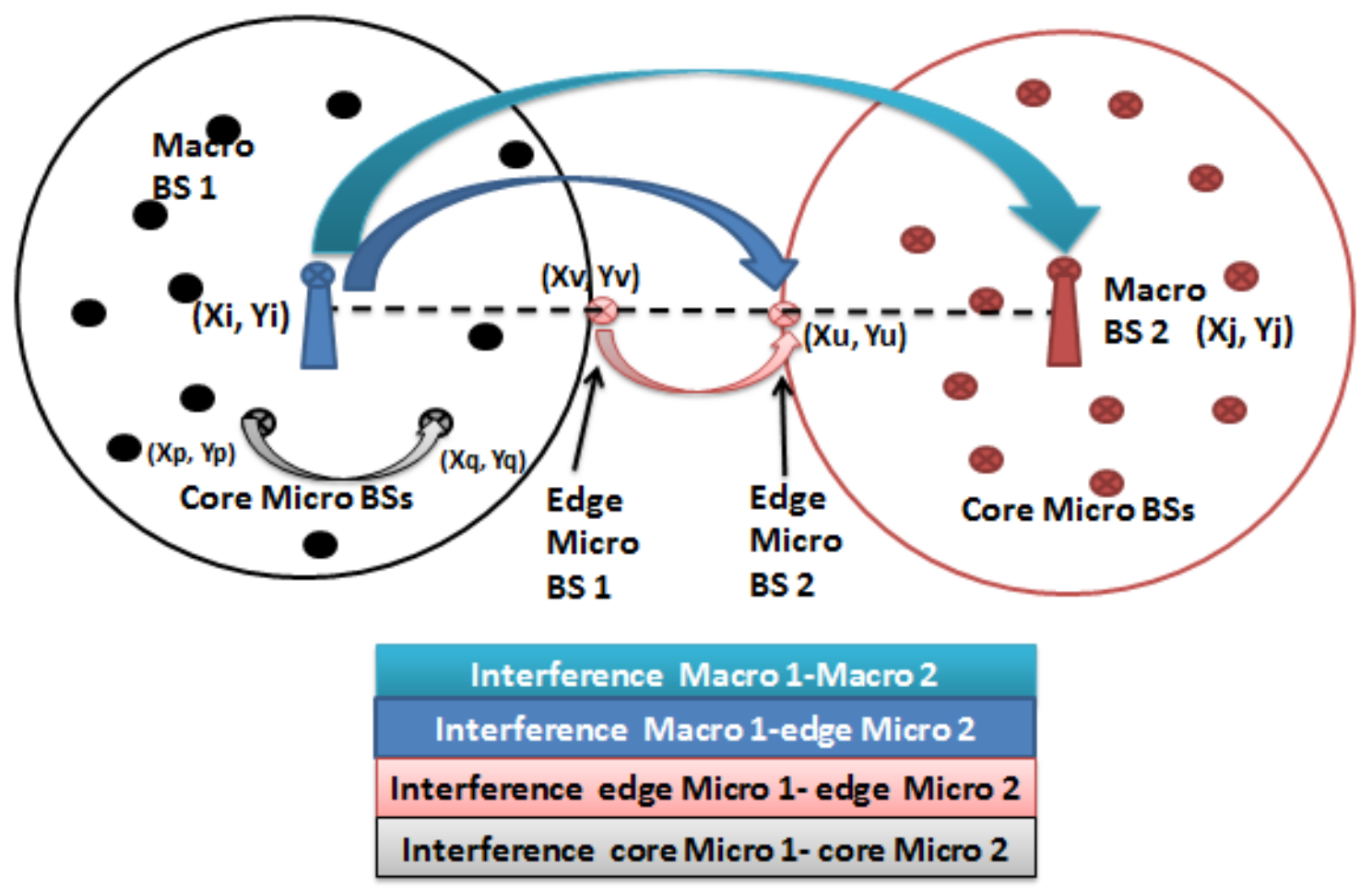

Figure 5.1: An explanation of various interference levels in our model.

Fig. 5.1 demonstrates various interference sources.

The interference between two Macro BSs $i$ and $j$ will be:

$$
I_{i j}=P_{M}\left(h_{i j}\right)^{2} d_{i j}^{-\alpha}
$$

where $h_{i j}$ is the fading gain between two Macro BSs, and considering $\left(X_{i}, Y_{i}\right)$ and $\left(X_{j}, Y_{j}\right)$ as the coordinates of the positions of the two Macro BSs, then the distance between them is given as $d_{i j}=\sqrt{\left(X_{i}-X_{j}\right)^{2}+\left(Y_{i}-Y_{j}\right)^{2}}$.

The interference from a Macro BS $i$ to a Micro BS $u$ at the edge of the adjacent cell is given by:

$$
I_{i u}=P_{M}\left(h_{i u}\right)^{2} d_{i u}^{-\alpha}
$$

where $h_{i u}$ is the fading gain between the Macro BS and edge Micro BS, and the distance between them is $d_{i u}=\sqrt{\left(X_{i}-X_{u}\right)^{2}+\left(Y_{i}-Y_{u}\right)^{2}}$.

The interference between two Micro BSs $u$ and $v$ located at the outer edges of two adjacent cells will be:

$$
I_{u v}=P_{m}\left(h_{u v}\right)^{2} d_{u v}^{-\alpha}
$$

where, $h_{u v}$ is the fading gain between the two edge Micro BSs, and the distance between them will be $d_{u v}=\sqrt{\left(X_{u}-X_{v}\right)^{2}+\left(Y_{u}-Y_{v}\right)^{2}}$. 
The interference between two Micro BSs $p$ and $q$ at the core of a given cell will be:

$$
I_{p q}=P_{m}\left(h_{p q}\right)^{2} d_{p q}^{-\alpha}
$$

where $h_{p q}$ is the fading gain between two core Micro BSs, and considering $\left(X_{p}, Y_{p}\right)$ and $\left(X_{q}, Y_{q}\right)$ as the coordinates of the positions of the two Macro BSs, then the distance between them is given as $d_{p q}=$ $\sqrt{\left(X_{p}-X_{q}\right)^{2}+\left(Y_{p}-Y_{q}\right)^{2}}$.

The interference between a Macro BS $i$ at any given cell and a Micro BS $p$ at the core of the same cell will be:

$$
I_{i p}=P_{M}\left(h_{i p}\right)^{2} d_{i p}^{-\alpha}
$$

where $h_{i p}$ is the fading gain between a Macro BS in a given cell and a Micro BS at the core of the same cell, and the distance between them is given as $d_{i p}=\sqrt{\left(X_{i}-X_{p}\right)^{2}+\left(Y_{i}-Y_{p}\right)^{2}}$.

\subsection{Optimization of Macro BSs Locations in HetNets}

\subsubsection{Dummy Intersecting Circle (DIC) Problem Formulation}

A common practice was that BSs in each tier of HetNets were usually randomly deployed according to a PPP. The locations chosen by a PPP may not be the best locations to achieve the highest signal power or the least interference. In this section, we first deploy BSs using a PPP, considering line of sight between BSs we optimize those locations to minimize (1) Macro-to-Macro BS interference, (2) Macro-to-Micro BS interference, and (3) Micro-to-Micro BS interference considering the downlink channel from the Macro BS to the Micro BSs. The key idea behind our suggested approach is that we assume an imaginary circular cell surrounding each Macro BS along with all its associated Micro BSs and, minimize the interference coming from all sources to the Micro BSs at the edge of the circle considering line of sight components. Then we can treat every Macro BS along with its associated Micro BSs as an independent unit. In other words, we loosely assume, a Micro BS at the core of the circle will not get interference from the neighboring Macro BSs or their associated Micro BSs.

Fig. 5.2 represents a network arrangement with six Macro BSs, where the middle BS on the upper row is the BS under consideration and it is surrounded by five interfering adjacent Macro cells. We have to eliminate interference from all the five adjacent BSs by applying the proposed algorithm. Our optimization problem 


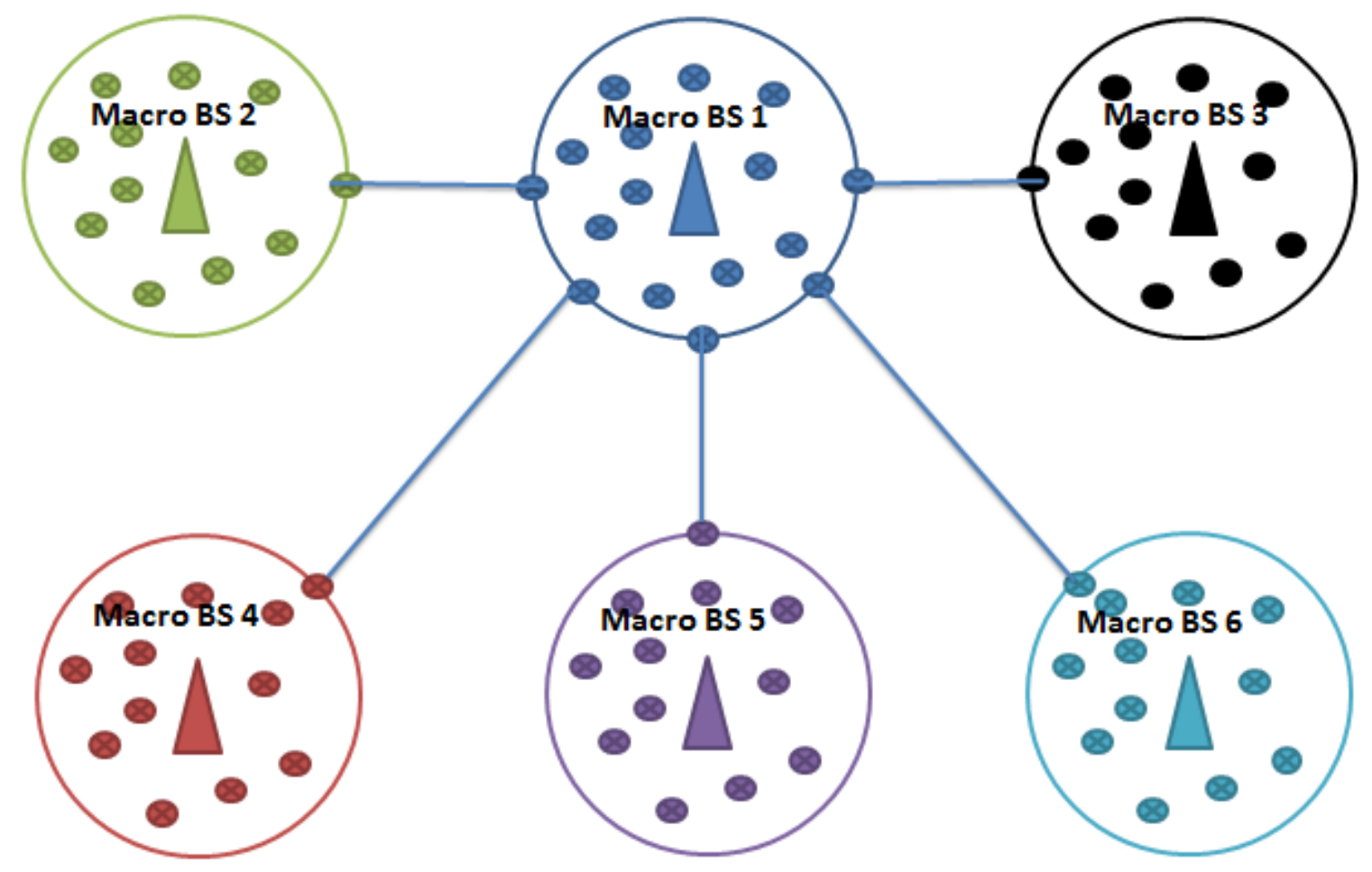

Figure 5.2: An illustration of considered scenario: $B S_{1}$ gets interference from surrounding BSs

will be formulated as:

$$
\begin{array}{ll}
\min & \sum_{i=1}^{I} \sum_{j=1, i \neq j}^{J} I_{i j}+\sum_{i=1}^{I} \sum_{u=1, i \neq u}^{U} I_{i u}+\sum_{u=1}^{U} \sum_{v=1, u \neq v}^{V} I_{u v} \\
\text { subject to } \quad & C 1: I_{i j} \leq \gamma_{1} \\
& C 2: I_{i u} \leq \gamma_{2} \\
& C 3: I_{u v} \leq \gamma_{3} \\
& C 4: 2 \cdot R \geq d_{i j}, \forall d_{i j} \in u[0, R] \\
& C 5: \sqrt{A} \geq X_{j} \geq-\sqrt{A}, \forall X_{j} \in u[-\sqrt{A}, \sqrt{A}] \\
& C 6: \sqrt{A} \geq Y_{j} \geq-\sqrt{A}, \forall Y_{j} \in u[-\sqrt{A}, \sqrt{A}]
\end{array}
$$

where, constraints $C_{1}, C_{2}$, and $C_{3}$ represent that the interference should be less than certain thresholds. Constraint $C_{4}$ represents the distance criteria between every two adjacent Macro BSs. Constraints $C_{5}$ and $C_{5}$ restrict the presence of the Macro BSs within the chosen working area where suggested values from the optimizer come from a uniform probability density function.

\section{Our algorithm works as follows:}

1. First, generate the locations of Macro and Micro BSs according to two dependent PPPs using a 


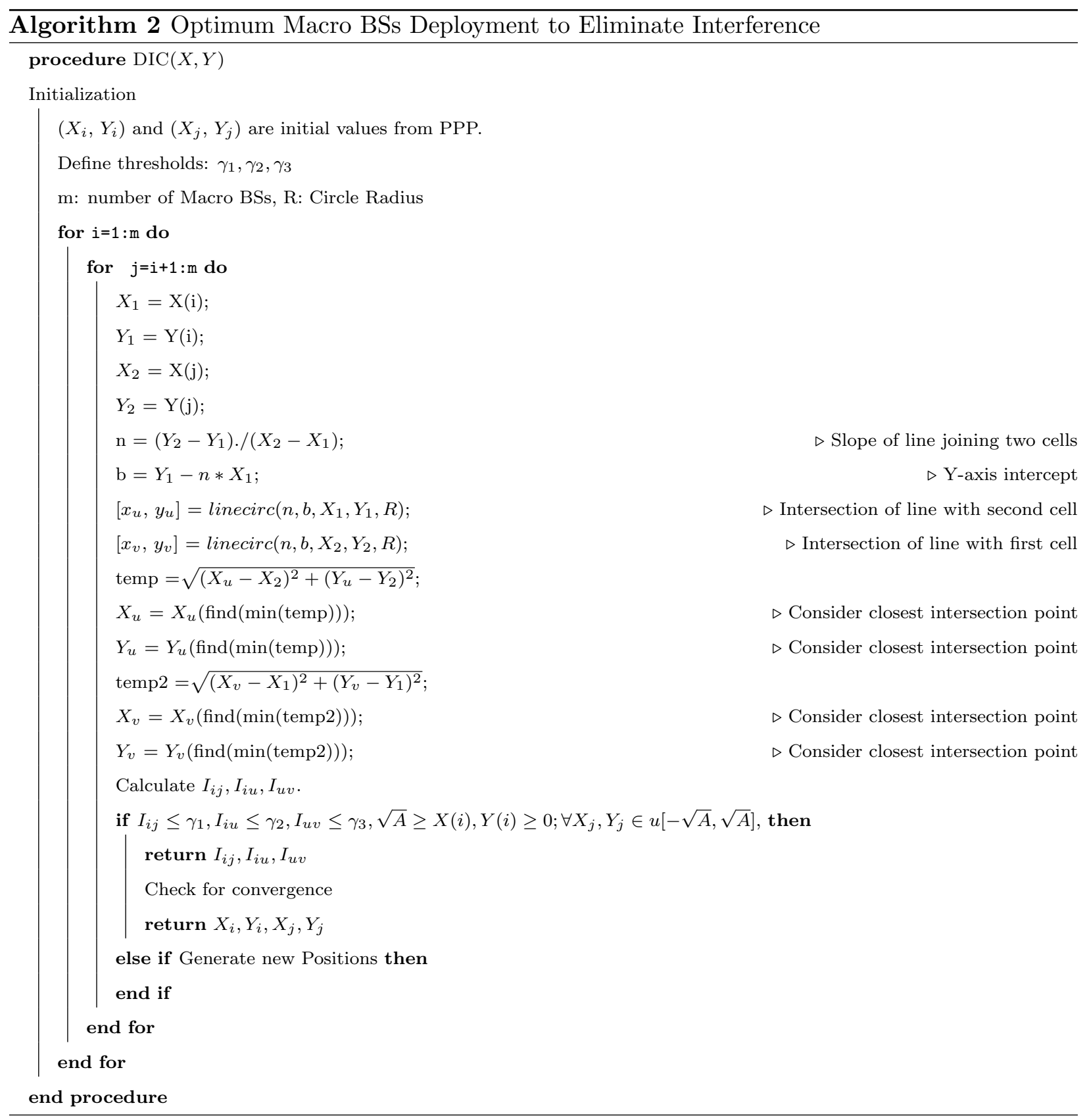


parent-child relationship.

2. Next, apply a thinning process to verify that the distance between every two Macro BSs is greater than a certain threshold. We do not want Macro BSs to overlap or be very close to each other. Also, We repeat the same process for the Micro BSs.

3. After applying the thinning process, we take the locations obtained from thinning and feed them as initial values to the optimization process.

4. Assume the presence of an imaginary circle surrounding every Macro BS and its associated Micro BSs. The generated circle is assumed to pass through an edge Micro BS on the circumference. To get the location of the dummy Micro BS at the edge, we draw a line from the center of the main Macro BS to all the neighboring interfering ones. The line will intersect each circle at two points. The smallest value will be considered and the other one will be discarded as we are not interested in the far edge Micro BS. The distances from the Macro BS to the edge Micro BS of the adjacent cell will be considered and used for interference calculation.

5. Interference coming from the core Micro BSs will not be considered and we consider interference at the edge Micro BS only. The assumption is that, if interference is eliminated at the neighboring edge Micro BS, then, there would be no interference coming from the neighboring core Micro BSs.

6. We minimize interference from all adjacent Macro BSs. Then, from neighboring Macro BSs to the edge Micro BS at the first circle. Finally, we minimize the interference between the edge Micro BSs of two adjacent circles. We repeat the same process until we cover all circles in the entire network.

The detailed DIC algorithm is explained in block Algorithm 2.

\subsubsection{Modified Dummy Intersecting Circle (MDIC) Problem Formulation}

We want to cover all sources of interference that a Macro cell may encounter to find a way to isolate it and treat it as an independent unit. Here we divide the Micro BSs associated with any Macro cell into core and edge Micro BSs. Edge Micro BSs will serve as a means of finding the location of the farthest point at the cell edge and minimize interference at it. We study the transmission between two Macro BSs in adjacent cells, the transmission from a Macro BS to any Micro BS in an adjacent cell (backhaul link), the transmission between two outer edge Micro BSs in adjacent cells, and the transmission between all Micro BSs at the core of a given Macro cell. Our algorithm is divided into two steps; first one is the Macro BSs optimum locations and second one is the Micro BSs optimum locations to eliminate all sources of interference. Our proposed algorithm is explained and split among blocks Algorithm 3 and Algorithm 4. Also, flow chart 5.3 further gives more explanation. 


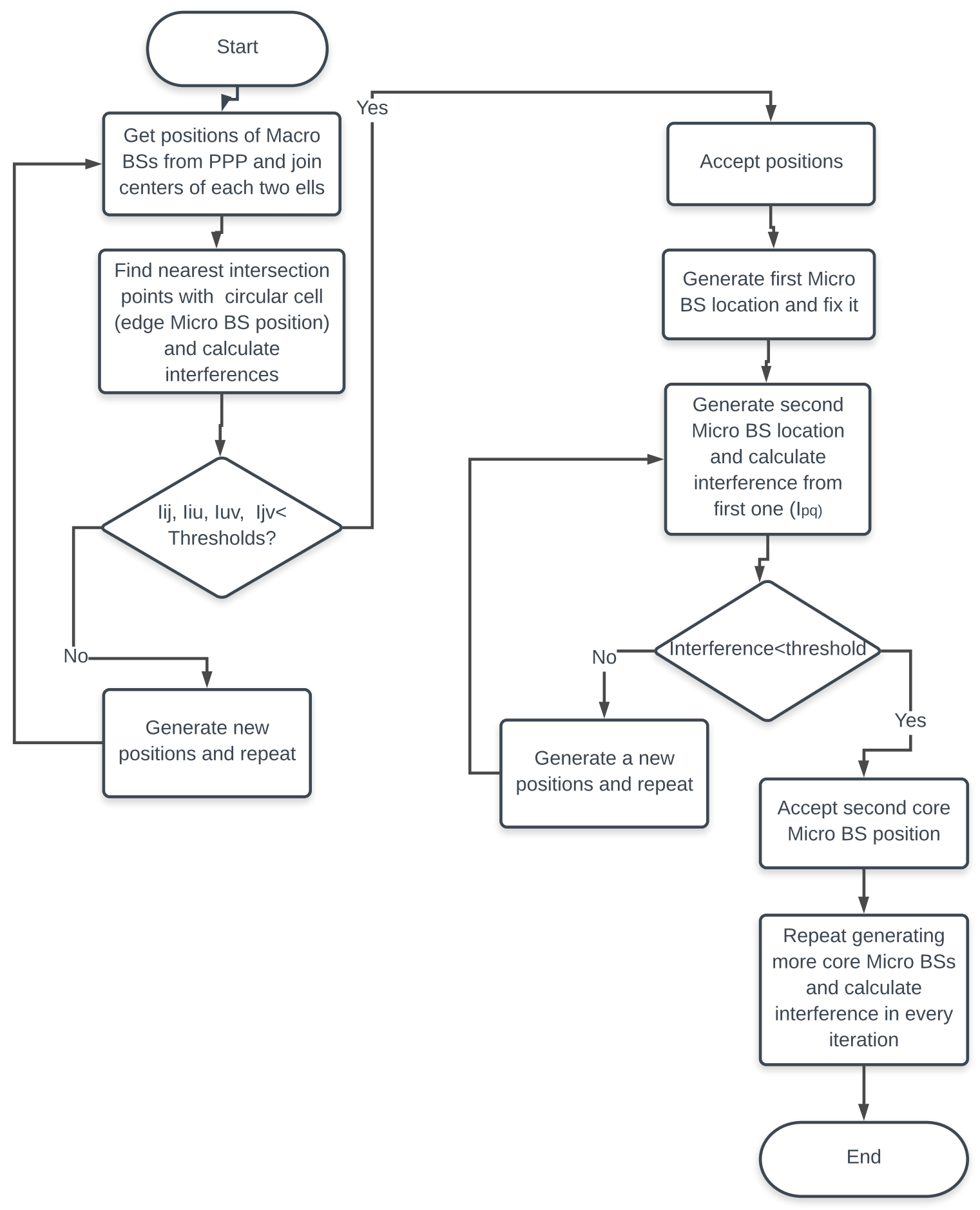

Figure 5.3: Flow chart of our proposed MDIC algorithm 
For the first step to find optimum Macro BSs locations, our optimization problem will be formulated as:

$$
\begin{aligned}
& \min _{\mathbf{X}, \mathbf{Y}}\left\{\begin{array}{l}
f_{1}=\sum_{i=1}^{I} \sum_{j=1, i \neq j}^{J}\left[I_{i j}\right] \\
f_{2}=\sum_{i=1}^{I} \sum_{u=1, i \neq u}^{U}\left[I_{i u}\right] \\
f_{3}=\sum_{j=1}^{J} \sum_{v=1, j \neq v}^{V}\left[I_{j v}\right] \\
f_{4}=\sum_{u=1}^{U} \sum_{v=1, u \neq v}^{V}\left[I_{u v}\right]
\end{array}\right. \\
& \text { Subject to }\left\{\begin{array}{l}
C 1: I_{i j} \leq \gamma_{1} \\
C 2: I_{i u} \leq \gamma_{2} \\
C 3: I_{u v} \leq \gamma_{3} \\
C 4: I_{j v} \leq \gamma_{4} \\
C 5: 2 \cdot R \geq d_{i j}, \forall d_{i j} \in u[0, R] \\
C 6: \sqrt{A} \geq X_{j} \geq-\sqrt{A}, \forall X_{j} \in u[-\sqrt{A}, \sqrt{A}] \\
C 7: \sqrt{A} \geq Y_{j} \geq-\sqrt{A}, \forall Y_{j} \in u[-\sqrt{A}, \sqrt{A}]
\end{array}\right.
\end{aligned}
$$

where, constraints $C_{1}, C_{2}, C_{3}$, and $C_{4}$ represent that the interference should be less than certain thresholds. Constraint $C_{5}$ represents the distance criteria between every two adjacent Macro BSs. Constraints $C_{6}$ and $C_{7}$ restrict the presence of the Macro BSs within the chosen working area where suggested values from the optimizer come from a uniform probability density function.

After avoiding all sources of interference from adjacent Macro cells, every Macro cell can be considered as an independent unit.

Secondly, we optimize Micro BSs locations, which are associated with every Macro BS. For the second part, our optimization problem will be:

$$
\begin{aligned}
& \min _{\mathbf{X}, \mathbf{Y}} f_{5}=\sum_{p=1}^{P} \sum_{q=1, p \neq q}^{Q}\left[I_{p q}\right] \\
& \text { Subject to }\left\{\begin{array}{l}
C 8: I_{p q} \leq \gamma_{5} \\
C 9: d_{c p} \leq d_{c p_{\text {min }}}
\end{array}\right.
\end{aligned}
$$

where constraint $C_{8}$ restrict the interference between Micro BSs in the same cell not to go over a certain threshold value. Also, constraint $C_{9}$ restricts that the minimum distance between the parent Macro BS and the associated Micro BSs should not exceed a certain value. 


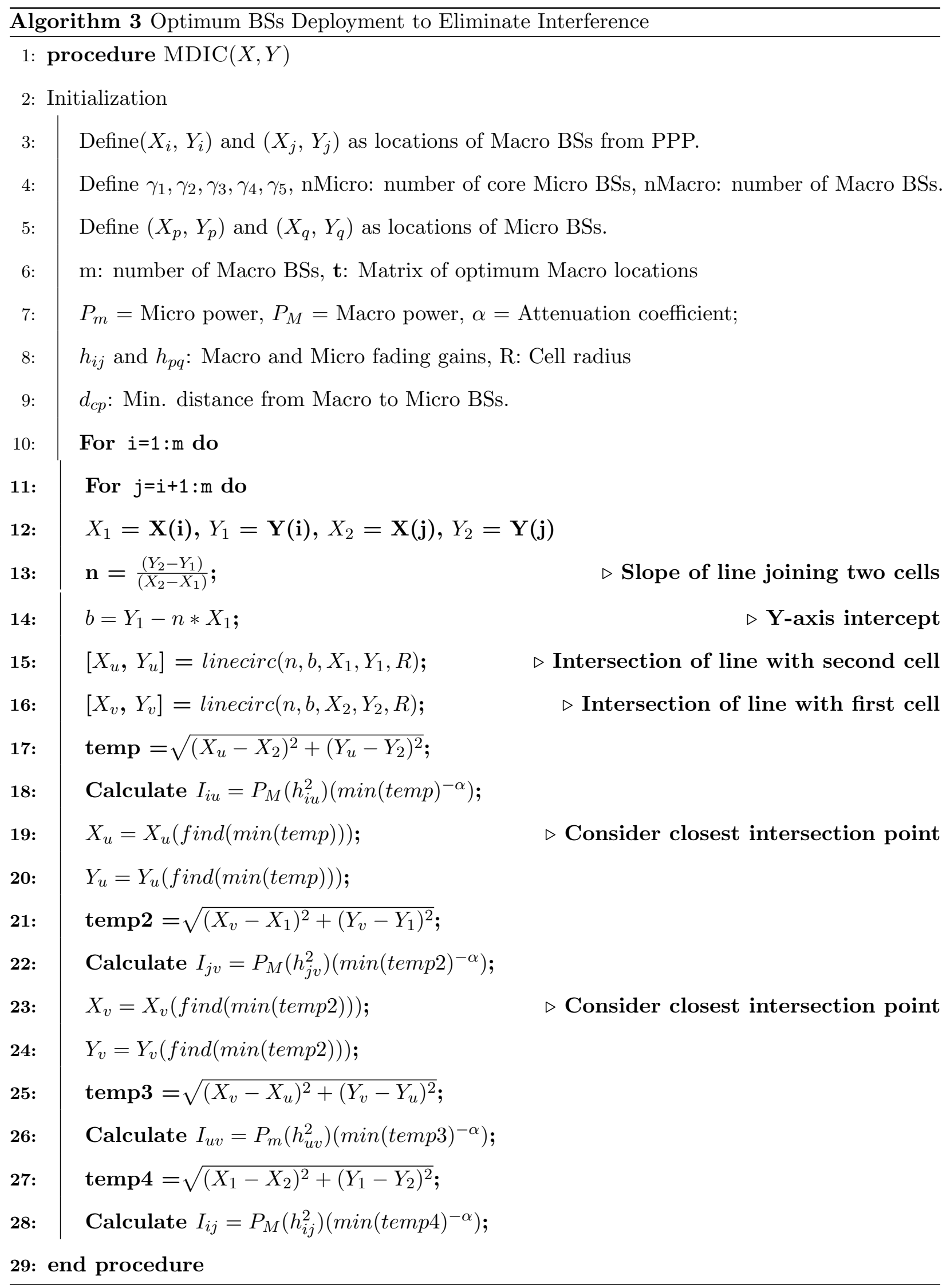




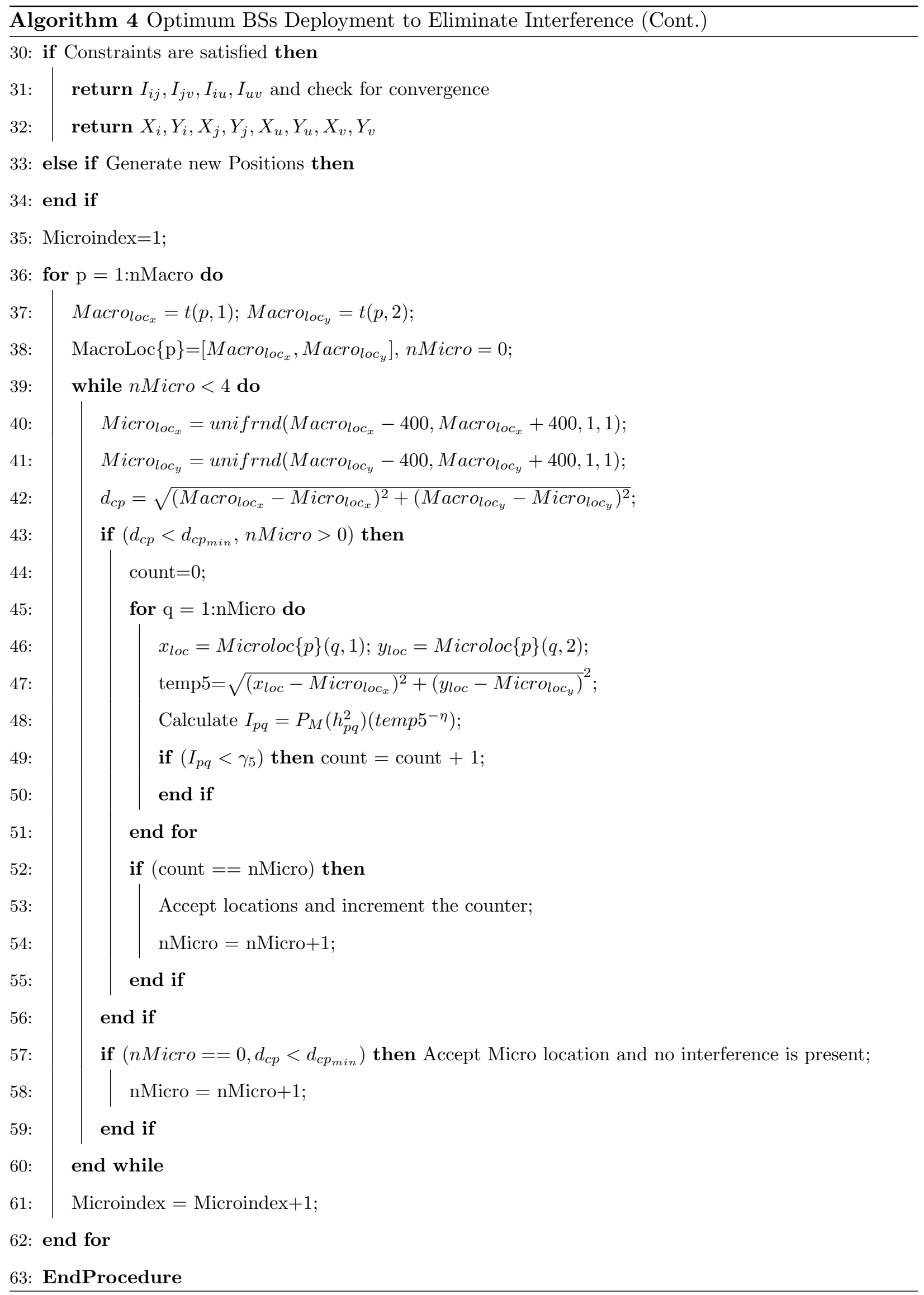




\section{Our algorithm works as follows:}

1. First, we generate uniform locations of Macro BSs from a PPP and assume a circular cell surrounding every Macro BS along with its associated Micro BSs.

2. Restrict distances between every two Macro BSs to be greater than a certain threshold and exclude too close locations. Those locations are initial values that we want to optimize for interference reduction.

3. Consider Micro BSs at the outer edge of the circular cell and we neglect core Micro BSs at this point. If interference is eliminated at the edge of the cell, then no interference will pass to the core.

4. Consider that each Micro BS at the circumference of each circular cell is facing an adjacent Micro BS at the circumference of the neighboring cell. To get the exact location of the edge Micro BS at each cell, we join the centres of every cell to all other adjacent ones ( connect the line between each Macro BS to its neighboring ones ). The line will intersect every circular cell at two locations, so we consider the closest one and we discard the far edge Micro BS. We consider distances between every two Macro BSs, every two Micro BSs, and between Macro and Micro BSs, which we apply for interference calculation.

5. We minimize interference between Macro BSs of two adjacent cells, between the edge Micro BSs of two adjacent cells, and from adjacent Macro BSs to an entire edge Micro BS. We repeat the same procedure for all the cells in the network and get optimum locations of Macro BSs.

6. After isolating every Macro cell from all sources of interference, we find the optimum Micro BSs locations in every cell to minimize interference from adjacent Micro BSs in the same cell (Note that we did not consider Micro BSs from adjacent cells as there is no inter cell interference now). We consider matrix $t$ containing all optimum Macro BSs locations, where Macro $_{l o c_{x}}$ represents all X Coordinates for the Macro BSs and Macro $_{l o c_{y}}$ represents all Y Coordinates.

7. For the first optimum Macro BS location, we generate a random location (Microloc, Micro $_{\text {loc }}$ ) for the first Micro BS from a uniform distribution. Restrict the first Micro BS to be within a certain distance

$\left(d_{c p_{\min }}\right)$ from the Macro BS and not to exceed it. For only one Micro BS there is no interference, so we accept the location of that Micro BS.

8. Generate a second Micro BS from a uniform distribution and restrict it to be within distance $d_{c p_{\text {min }}}$ from the Macro BS. Calculate interference between the first and the second Micro BSs. If interference is less than a certain threshold, then we accept the second Micro BS location. Otherwise, we reject it and generate a new location and repeat the same process again and apply the constraints.

9. Repeat for all $C$ Micro BSs and calculate all interference values and obtain their optimum locations.

10. Repeat from step 7 to cover all Macro cells. 


\subsection{Performance Analysis}

Macro BSs, Micro BSs, and UEs are scattered uniformly in the working area according to three homogeneous dependant PPP using parent-child relation-ship. Matlab ${ }^{T M}$ software was used to execute the simulation for our proposed mathematical model and Table 5.1 presents the parameter values that we used. First, we applied a thinning process, where the locations of BSs were randomly distributed according to a PPP. The BSs which do not satisfy a predefined distance criteria were excluded. Then, UEs which correspond to every Micro BS were generated as well according to a dependant PPP using a parent-child relationship and the thinning process was applied to them. The locations of the generated Macro BSs were assigned and fed into the optimization code to optimize their values. We used Matlab ${ }^{T M}$ optimization toolbox, which helped us to suggest new distances in every iteration restricting them to be generated from a uniform distribution. We use interior-reflective Newton optimization method, which is based on a subspace trust region method. We used 'fmincon' optimization function to solve this constrained nonlinear minimization problem.

Fig. 5.4 presents a comparison between the total interference $\left(I_{i j}+I_{u i}+I_{u v}\right)$ obtained before and after applying the DIC algorithm. It is obviously seen that interference is reduced when BSs are placed at the suggested optimum locations. Interference is less than half the initial value and we believe that interference can be almost eliminated using the suggested approach if the proper interference thresholds are initially chosen. Fig. 5.5, shows a comparison for the pictures of three Macro cells before and after applying MDIC algorithm. Every Macro BS as it emits the signal is represented by the bright white spot and signal power deteriorates with distance (represented by the grey shaded circular pattern around each cell). In 5.5a, interference is

Table 5.1: Parameters used for Simulation

\begin{tabular}{|c|c|}
\hline Parameter & Value \\
\hline$B_{n}$ (Number of Macro BS \\
Antennas) & 64 \\
\hline$A_{n}$ (Number of Micro BS \\
Antennas)
\end{tabular}




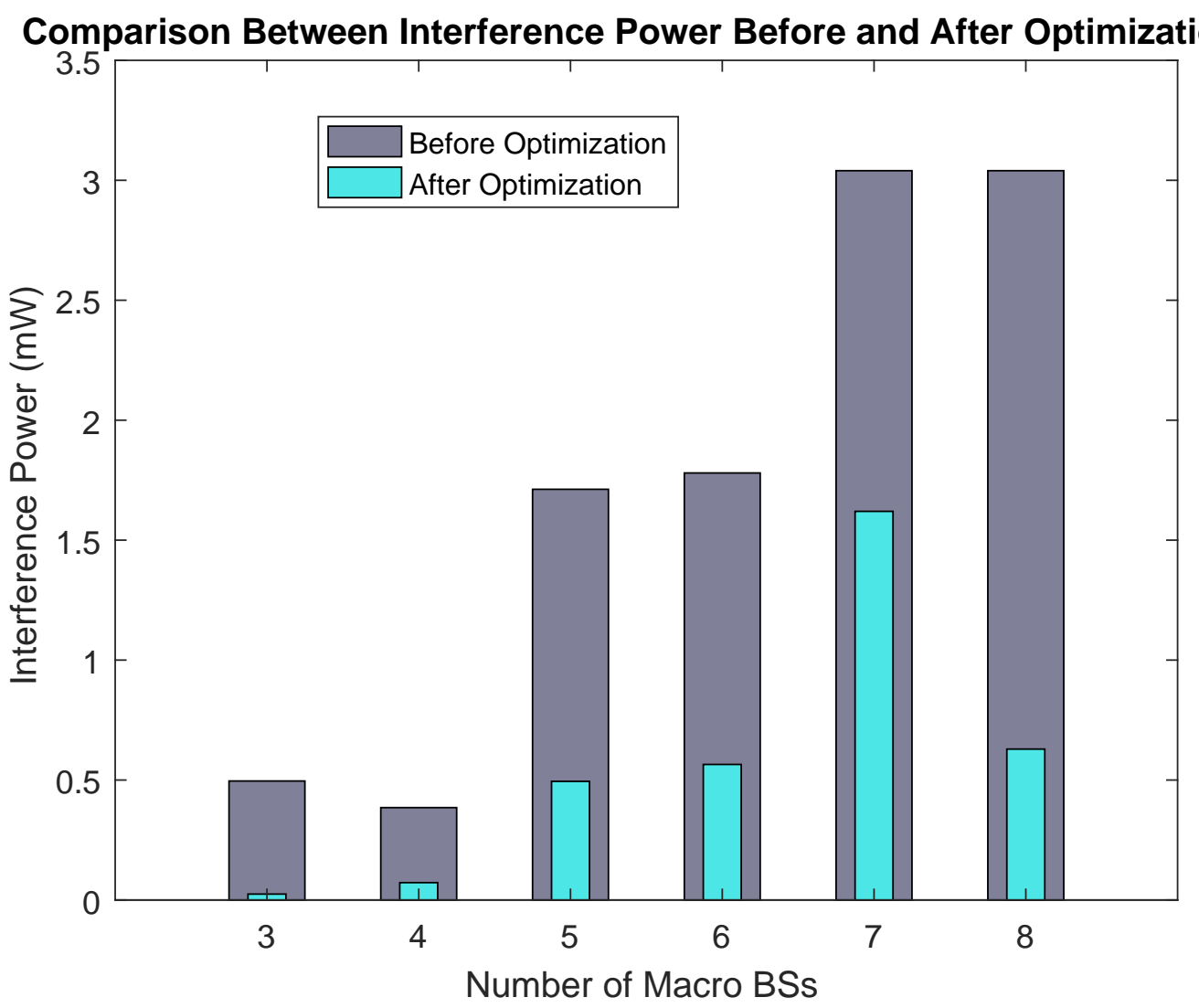

Figure 5.4: The total interference: Sum of (Macro-Macro), (Macro-Micro) and (Micro-Micro) interference before and after optimization

affecting badly among adjacent cells, while in 5.5b after applying the suggested MDIC algorithm every Macro cell is independent and interference free. Fig. 5.6, demonstrates the interference power from adjacent Micro BSs at three Macro cells. Assuming that we have four Micro BSs in each Macro cell, we can see that interference from all adjacent Micro BSs is kept below a certain threshold of $25 \mathrm{dBm}$. As long as interference is kept low, we can accept locations of the Micro BSs and consider them as the best solution to minimize interference.

\subsection{Sensitivity Analysis for Threshold Values}

In this Section, we consider changing the values of the thresholds $\left(\gamma_{1}, \gamma_{2}, \gamma_{3}\right.$, and $\left.\gamma_{4}\right)$ and studying their effect on the total sum of interference from all BSs in the network. Fig. 5.7 shows all threshold combinations that we have used for MDIC algorithm. Usually we vary one threshold and fix the others to get a better idea of how it behaves. In Fig 5.10, we are changing the threshold $\gamma_{1}$ for $I_{i j}$ (Macro base station-to-Macro base 
station) at: $[1.3,0.9,0.7,0.5,0.3,0.1,0.01]$ watts and fixing other two thresholds at $\gamma_{3}=0.01$ watt, $\gamma_{2}, \gamma_{4}=0.2$ watt. The first combination represents sum of interferences $\left(I_{i j}+I_{j v}+I_{i u}+I_{u v}\right)$ for all BSs coming from all adjacent cells before optimization for seven BSs. For other combinations from 2 to 7 , they represent the threshold values shown in Fig 5.7 after optimization. Before optimization, interference level is very high as some positions are so much deviated and contributing to this high interference level. In Fig. 5.11, we excluded the values before optimization to show variations in values with different combinations in more details. In general, we can consider sum of interference as decreasing with reducing the threshold value chosen. Fig. 5.12, presents sum of interference levels when changing $\gamma_{2}$, and $\gamma_{4}$ at: $[0.01,0.05,0.1,0.2,0.3]$ watts . Threshold combinations are presented in Fig.5.8. Interference sum is generally decreasing with increasing the thresholds, but when the chosen thresholds are very low, interference increases. Also, Fig. 5.13, presents sum of interference levels when changing $\gamma_{3}$ at: $[0.001,0.005,0.01,0.02,0.1]$ watts . Threshold combinations are presented in Fig.5.9. Interference sum is generally increasing with increasing the threshold. It is worth to mention that as interference before optimization is very high, any threshold level reduce interference by a sufficient amount. For the iterative approach or second stage of optimization for core Micro BSs locations. Fig. 5.14 shows comparison of sum of interference $\left(I_{12}+I_{13}+I_{23}+I_{14}+I_{24}+I_{34}\right)$ for core Micro BSs for seven BSs using two threshold levels $\left(\gamma_{5}=0.005,0.008\right)$ levels. As expected, when the chosen threshold is higher, the sum of interference is higher and vice versa as this is an iterative approach.

\subsection{Chapter Summary}

HetNets are gaining the attraction of the communication society as they work in multi-layers cooperating together to fulfill the dream of connecting the globe as one big network in the future. From stochastic geometry, HetNets are modeled with uniform distributions according to a PPP. However, the chosen positions from the PPP are not the optimum locations to eliminate all sources of interference.

In this chapter, we introduce a novel algorithms for Macro BSs deployment in HetNets by minimizing sum of interference from all adjacent Macro and Micro cells, considering imaginary circles surrounding all the Macro BSs along with their associated Micro sets. We treated every circle as an independent unit that is not affected by adjacent Macro and Micro BSs interference. Also, we considered an algorithm for optimum Micro BSs locations within every Macro cell to minimize inter and intra cell interference. Our results are feasible in the real wireless network systems that would help in the development of future 5G systems. 

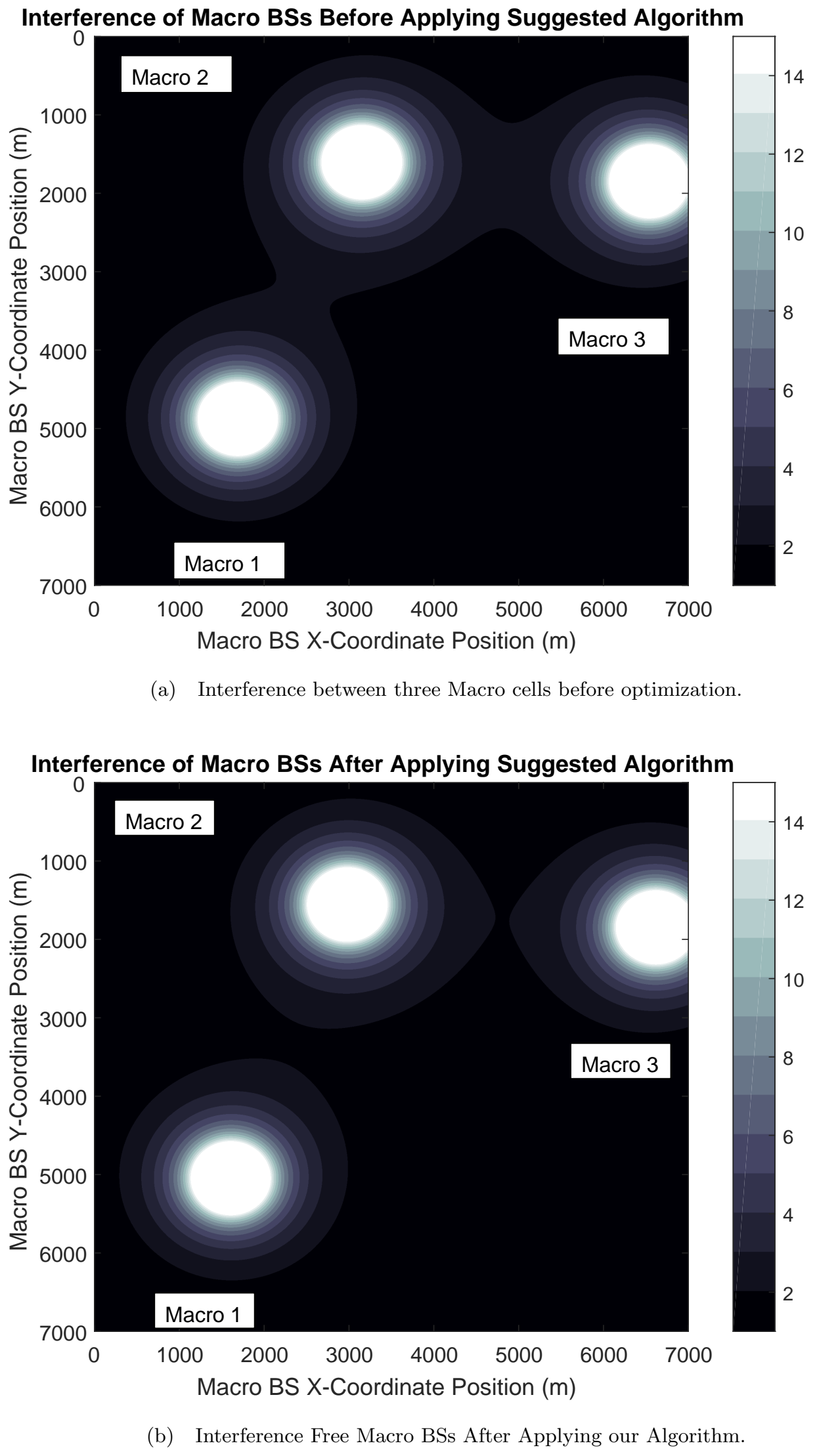

Figure 5.5: An illustration of how Macro cells became independent units and interference free. 


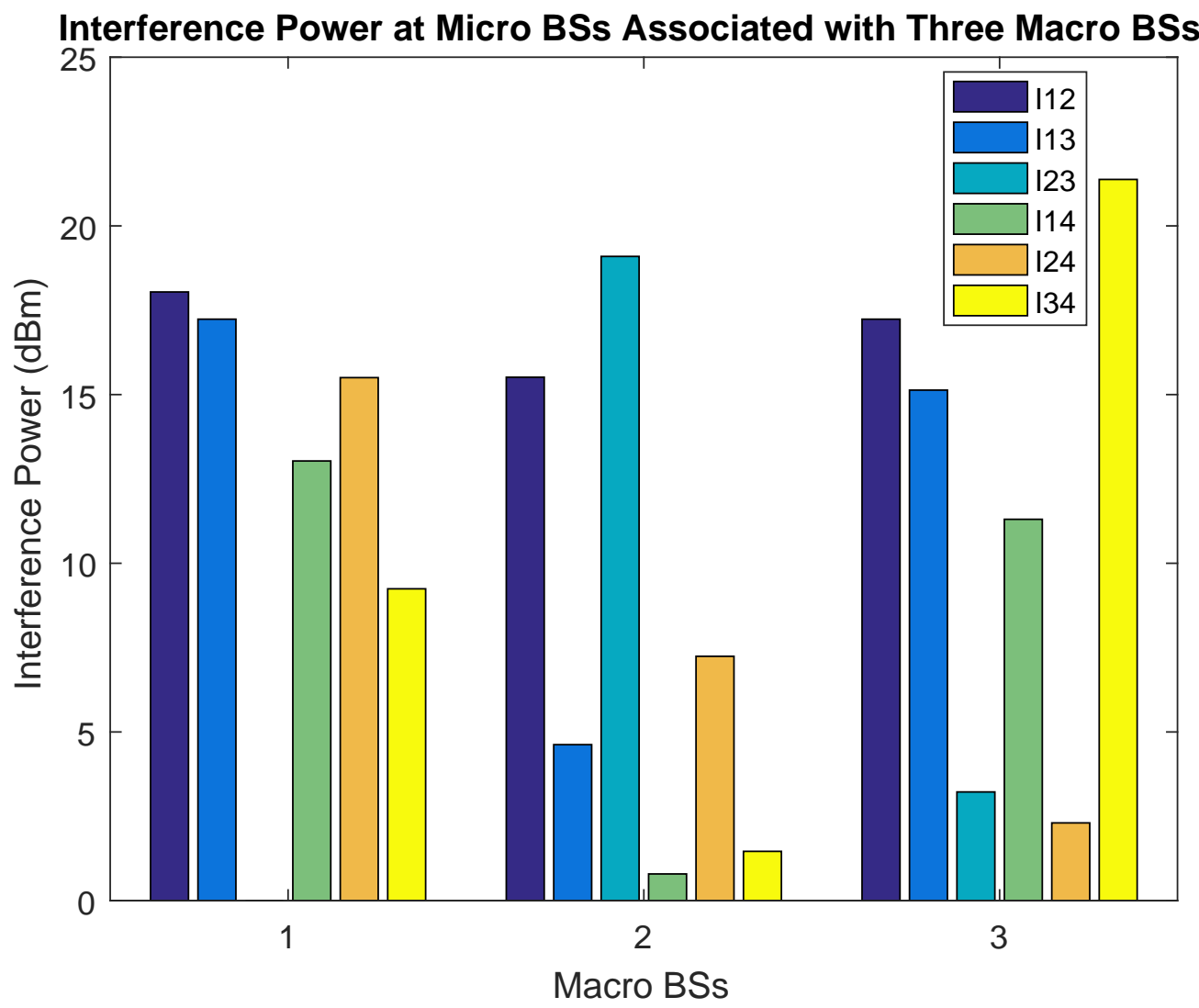

Figure 5.6: Interference power between Micro BSs associated with every Macro BS

\begin{tabular}{|l|l|}
\hline \multicolumn{1}{|c|}{ Changing $\gamma^{1}$, fixing others } & Combinations \\
\hline$\gamma 1=1.3 ; \gamma 3=0.01 ; \gamma 2$, and $\gamma 4=0.2$ & First combination \\
\hline$\gamma 1=0.9 ; \gamma 3=0.01 ; \gamma 2$, and $\gamma 4=0.2$ & Second combination \\
\hline$\gamma 1=0.7 ; \gamma 3=0.01 ; \gamma 2$, and $\gamma 4=0.2$ & Third combination \\
\hline$\gamma 1=0.5 ; \gamma 3=0.01 ; \gamma 2$, and $\gamma 4=0.2$ & fourth combination \\
\hline$\gamma 1=0.3 ; \gamma 3=0.01 ; \gamma 2$, and $\gamma 4=0.2$ & fifth combination \\
\hline$\gamma 1=0.1 ; \gamma 3=0.01 ; \gamma 2$, and $\gamma 4=0.2$ & sixth combination \\
\hline
\end{tabular}

Figure 5.7: Threshold combinations changing $\gamma_{1}$ related to Fig. 5.10 and Fig. 5.11. 


\begin{tabular}{|l|l|}
\hline Changing $\gamma 2$ and $\gamma 4$, fixing others & Combinations \\
\hline$\gamma 1=0.1 ; \gamma 3=0.1 ; \gamma 2$, and $\gamma 4=0.01$ & First combination \\
\hline$\gamma 1=0.1 ; \gamma 3=0.1 ; \gamma 2$, and $\gamma 4=0.05$ & Second combination \\
\hline$\gamma 1=0.1 ; \gamma 3=0.1 ; \gamma 2$, and $\gamma 4=0.1$ & Third combination \\
\hline$\gamma 1=0.1 ; \gamma 3=0.1 ; \gamma 2$, and $\gamma 4=0.2$ & fourth combination \\
\hline$\gamma 1=0.1 ; \gamma 3=0.1 ; \gamma 2$, and $\gamma 4=0.3$ & fifth combination \\
\hline
\end{tabular}

Figure 5.8: Threshold combinations changing $\gamma_{2}$ and $\gamma_{4}$ related to Fig. 5.12.

\begin{tabular}{|l|l|}
\hline Changing $\gamma^{3}$ fixing others & Combinations \\
\hline$\gamma 1=0.1 ; \gamma 3=0.001 ; \gamma 2$, and $\gamma^{4}=0.2$ & First combination \\
\hline$\gamma 1=0.1 ; \gamma^{3}=0.005 ; \gamma 2$, and $\gamma 4=0.2$ & Second combination \\
\hline$\gamma 1=0.1 ; \gamma^{3}=0.01 ; \gamma 2$, and $\gamma 4=0.2$ & Third combination \\
\hline$\gamma 1=0.1 ; \gamma 3=0.02 ; \gamma 2$, and $\gamma^{4}=0.2$ & fourth combination \\
\hline$\gamma 1=0.1 ; \gamma 3=0.1 ; \gamma 2$, and $\gamma 4=0.2$ & fifth combination \\
\hline
\end{tabular}

Figure 5.9: Threshold combinations changing $\gamma_{3}$ related to Fig. 5.13. 


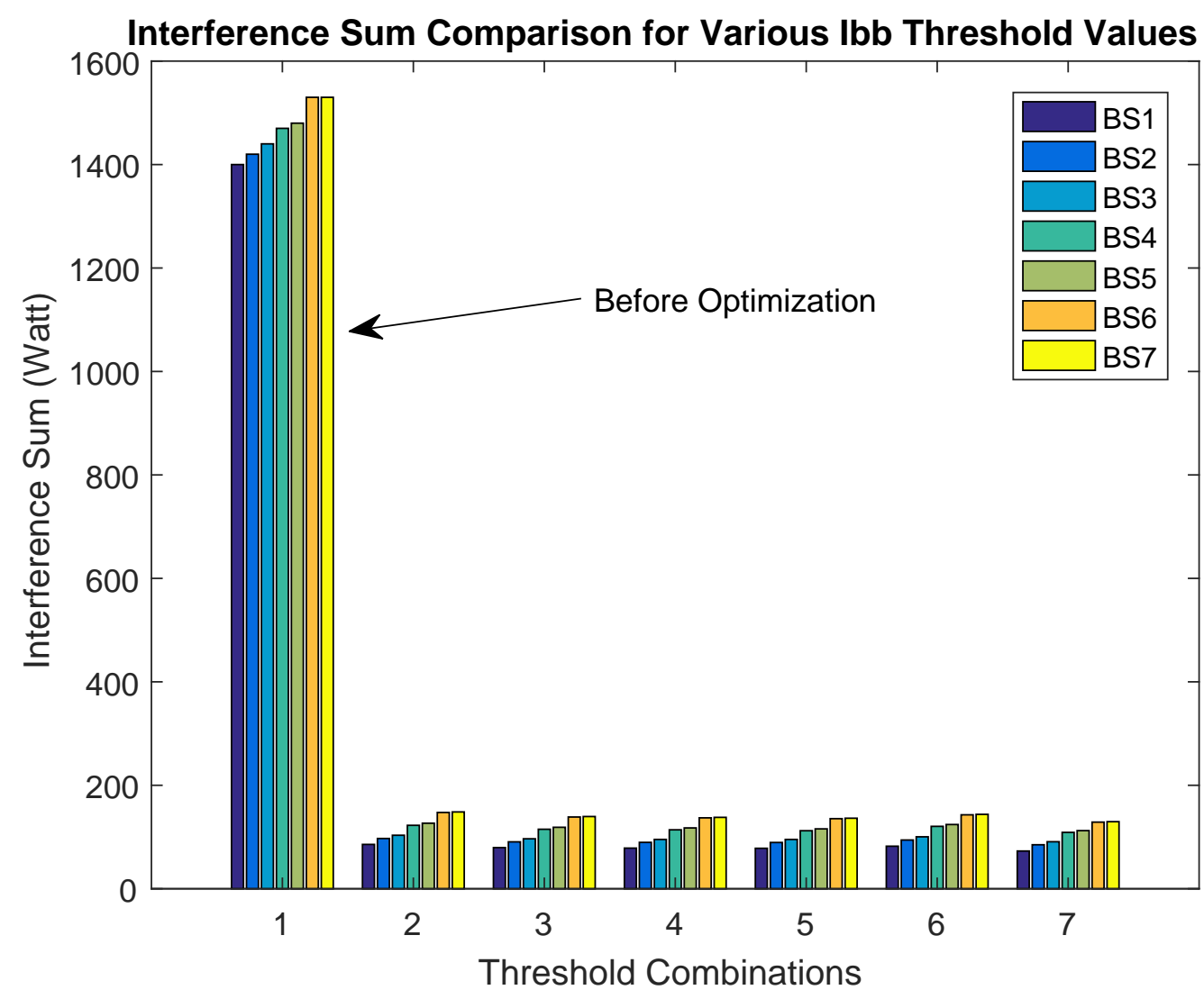

Figure 5.10: Sum of interference in the whole network before and after optimization changing $\gamma_{1}$. 


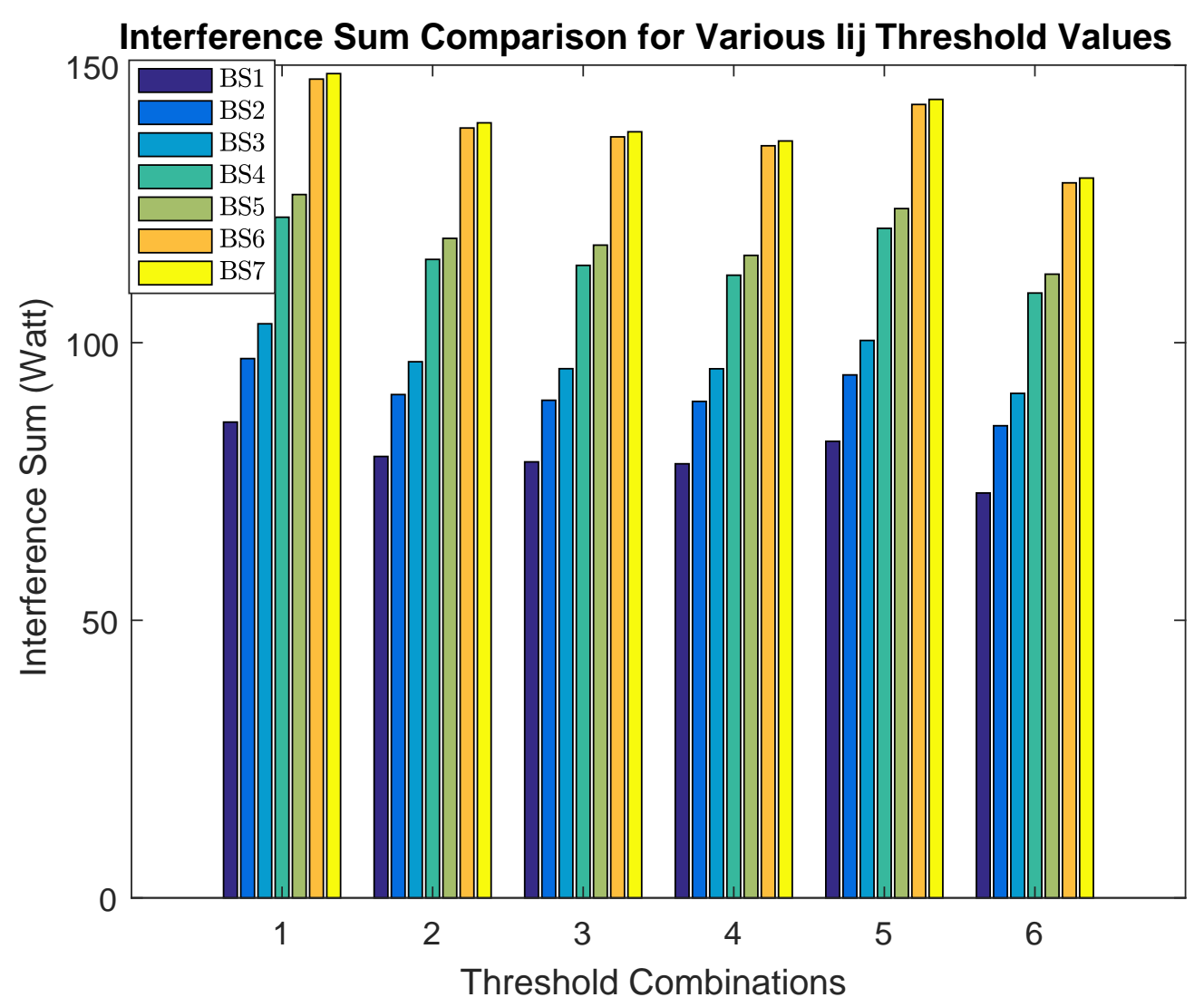

Figure 5.11: Sum of interference in the whole network with various $\gamma_{1}$ values. 


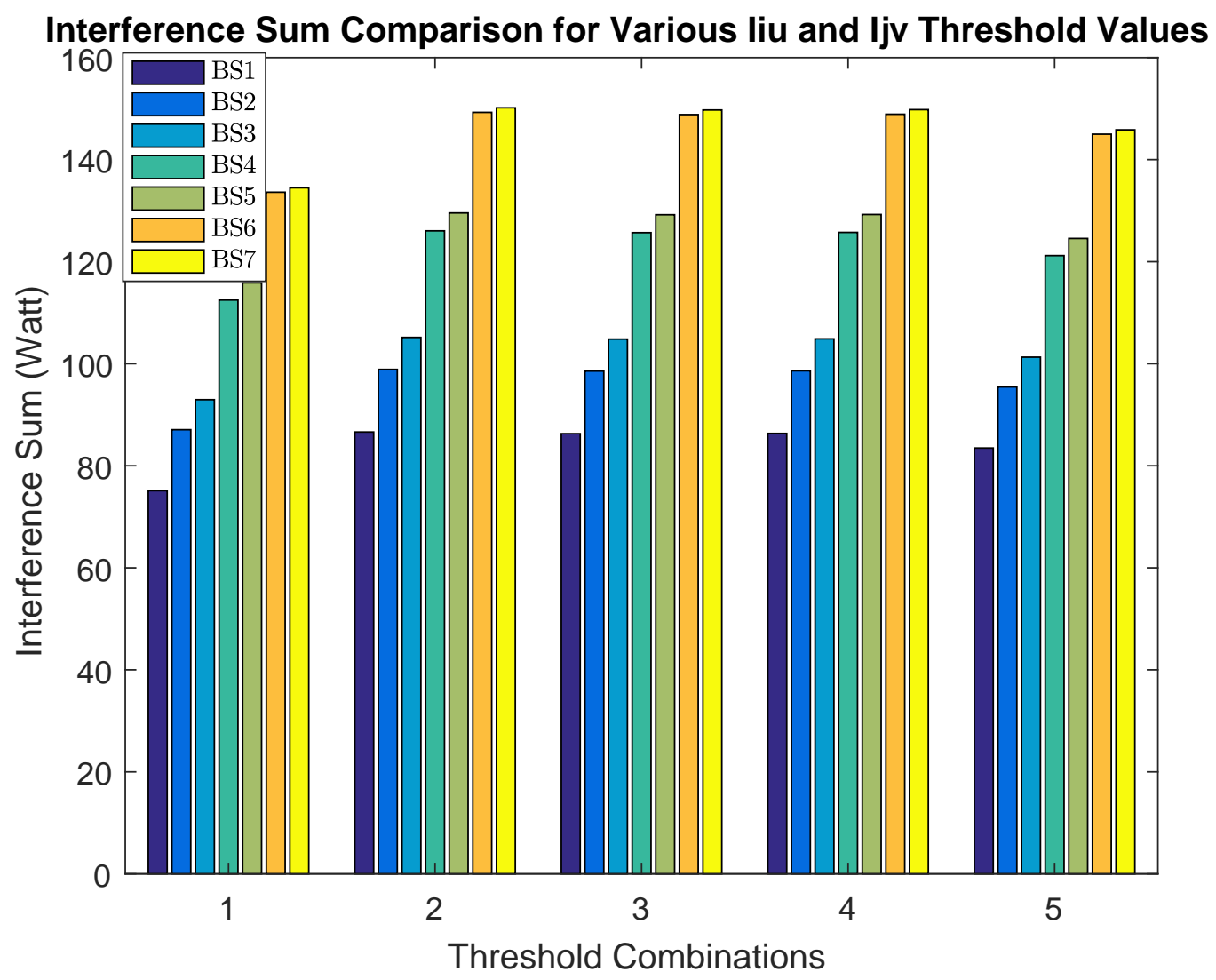

Figure 5.12: Sum of interference in the whole network changing $\gamma_{2}$ and $\gamma_{4}$. 


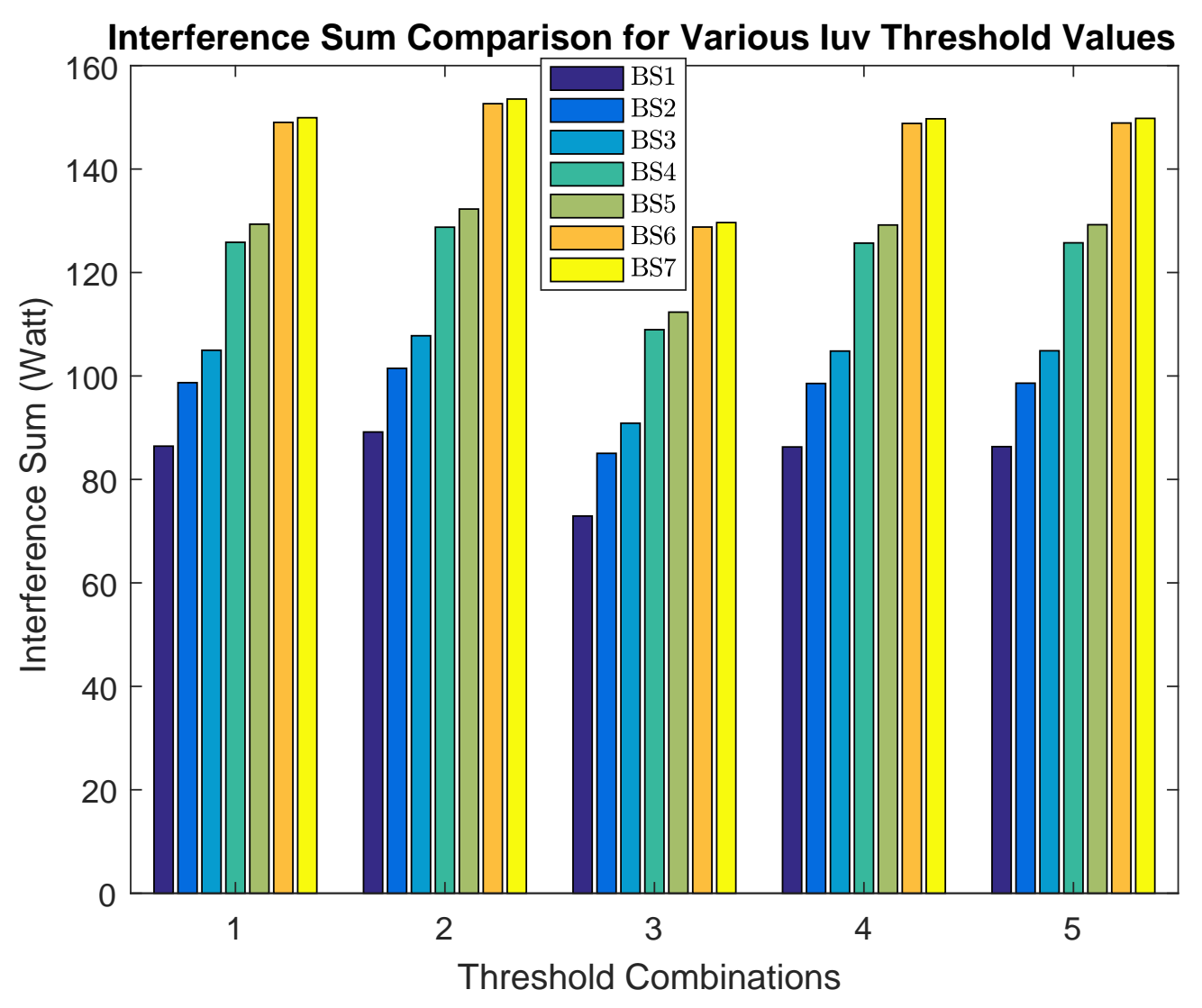

Figure 5.13: Sum of interference in the whole network changing $\gamma_{3}$. 


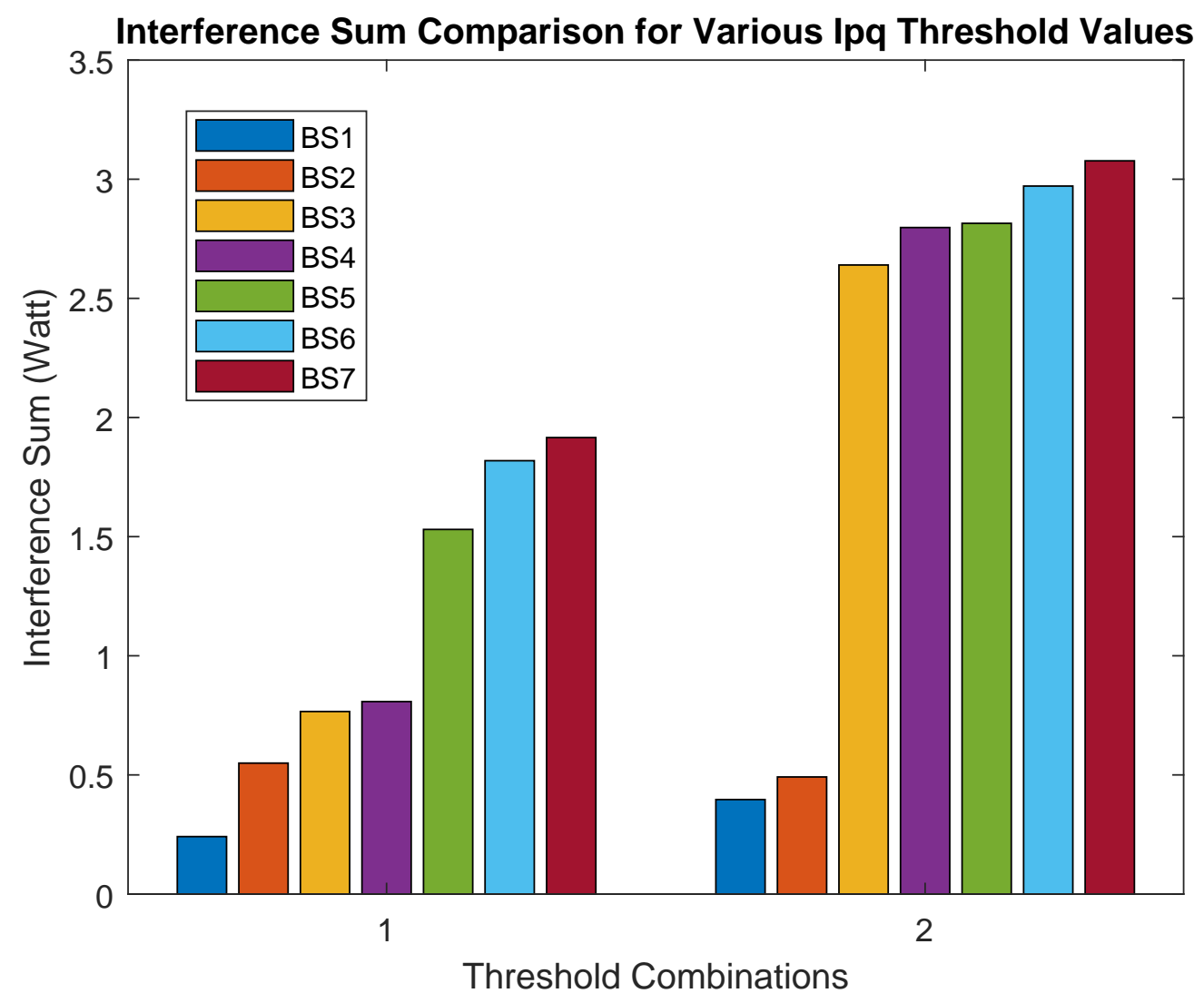

Figure 5.14: Interference sum comparison for two threshold levels, '1' refers to $\gamma_{5}=0.005$ watts and '2' refers to $\gamma_{5}=0.008$ watts. 
CHAPTER 6

\section{Load Distribution Standard Deviation (LSTD) User}

\section{Association Algorithm}

\subsection{Introduction}

The introduction of multi-tier HetNets is a good solution to enhance network performance [176]. The new system modeling is moving rapidly towards multi-dimensional concept with multiple layers working together [139] to improve the overall sum-throughput gain and to reduce the NMSE. Instead of growing the network horizontally in one dimension with complex designs, the network is growing vertically to reduce signaling overhead, increase energy efficiency, and improve data rate [140].

A proper UE association algorithm for HetNets is a great challenge, as the conventional maximum SINR UE association algorithm does not provide fair distribution of load when multiple tiers with varying BSs transmission powers are applied. Modern BSs need to cater multiple types of users in the multi-tier networks. However; when this is applied in HetNets, usually the Macro BS will win the game. Most of the UEs will be allocated to the Macro BS as it delivers a higher power level than the rest of the small cell BSs. Leaving the Micro BSs with minimal load, reducing the importance of multiple tiers in general.

\subsection{Related Work}

We noticed that several algorithms were developed based on maximizing SINR [174], weighted sum energy efficiency [177], sum rate [178], per-user utility function [179], and UE satisfaction [151].

Other approaches for UE association work on the idea that UEs are selfish and try to capture the 
maximum allowable bandwidth without considering other UEs needs $[141,180,181]$. UEs select the BS with the least connected UEs to it (least load) to get the rest of the available bandwidth for itself. The aforementioned selfish approach causes bandwidth imbalance in the network and failure in some links. Also, this algorithm does not consider overall network balance.

\subsection{Contributions and Organization}

In this chapter, we worked on rebalancing UE load distribution at all BSs in the network, starting initially from an acceptable SINR UE-BS association matrix until a uniform distribution at all BSs is achieved to ensure that all selected BSs possibilities for every UE provide an adequate SINR level. In conventional UE association algorithms, either SINR level or load per BS are considered for decision making. Whereas, in our proposed two algorithms both SINR level and network load are taken into consideration.

We investigate a new centralized UE association algorithm based on optimizing UE distribution at each BS by minimizing the standard deviation of the network load distribution. Initial BS load is defined based on a certain SINR threshold and all allocation possibilities are determined. UE attempts connecting to one of the possible BSs that has the least load. Standard deviation is calculated in the first iteration for the BSs with least loads for all UEs. If results are not satisfactory, then first UE checks the next available BS along with BSs with least loads for the rest of the other UEs and so on for the rest of the iterations. Optimum load distribution and fair balance are achieved in the network.

The rest of this chapter is organized as follows: Section 6.4 presents the description of LSTD user association algorithm. In Section 6.5, we present our objective function and present a detailed explanation of the algorithm. Section 6.6 shows the results of our proposed algorithm and Section 6.7 concludes this chapter.

\subsection{Explanation of the Proposed LSTD Algorithm}

In this section, we propose a new UE association technique in a HetNet open access system, where each UE can access any BS in any tier. First, we distribute users based on an SINR threshold (an acceptable level of SINR). In our proposed algorithm, we take network load into consideration and at the same time initially associate UEs based on an acceptable SINR level to make sure that good signal quality is maintained while load is redistributed. To implement this algorithm, initial SINR from all UEs to each BS is required, which in turn requires the knowledge of channel gains, attenuations, and transmission powers for every BS. In our suggested algorithm, we assign UEs to BSs minimizing the overall load standard deviation (Standard Deviation (Std)) of the network to reduce fluctuation in every BS load from the mean value. Standard deviation means how far measurements are deviated from the mean value. The mean value represents $\mathbb{E}\left[L_{k}\right]$ 
obtained from (4.49). If standard deviation is high, this means that distribution is not appropriate. We use standard deviation as a measure to identify how many base stations are overloaded and under loaded and redistribute them. If number of users and number of base stations are fixed then mean value will be fixed in every iteration, but standard deviation depends on distribution, so when distribution changes standard deviation will change as well.

For example, if we have 100 users and 10 base stations, then the ideal distribution is to give 10 users to every base station. In that case standard deviation will be zero. but in actual case this is not possible. We try to reach the standard deviation of zero if possible.

Our algorithm is explained in detail in blocks Algorithm 5 and Algorithm 6, and the flow chart in Fig. 6.4 gives a brief idea of the basic concept.

\subsection{Problem Formulation and Complexity Analysis}

Fig. 6.1 highlights our suggested UE-association algorithm, where the upper part shows all the available BS allocation possibilities based on a chosen SINR threshold $\left(\gamma_{t h}\right)$. The middle part shows how the load distribution was imbalanced before applying our algorithm. Finally, the last part, shows the optimum load distribution after applying our algorithm. Fig. 6.2 describes the basic idea of the suggested user association algorithm, where the sorted users are iteratively associated with the possible BSs all at once. The first column having $\mathrm{BS}$ indices as $(3,7,8)$ represents the possible BSs for association for the third UE and the successive columns are the possibilities for the rest of the UEs considering the BSs with less loads. Fig. 6.3 shows an explanation of the selection criteria of the best BS for a certain UE from all available combinations. We choose the UE with least number of available BSs for connection, then try all BSs possibilities along with the BS with the least load for the other UEs then calculate standard deviation and choose the best combination that shows a standard deviation below a certain threshold.

Fig. 6.5 shows a sample of user distribution and corresponding standard deviation in each iteration. Assuming network load or number of UEs per BS as (udist). It is worth to mention that udist is just a summation of load in every BS and does not reflect which user is associated to which BS in every iteration so standard deviation can be duplicated despite that distribution is different. We can see that there are 14 BSs and $100 \mathrm{UEs}$, so mean value is 7.1428 and it is fixed in all iterations and approximated to 8 . For the first iteration distribution, standard deviation is calculated by adding squares of the distances from the mean, dividing by the number of points, then taking the square root. Fig. 6.6 shows all transitional iterations as user attempts the connection to all possibilities. Heavily loaded BSs are loosing part of their load and under loaded BSs are gaining more load along iterations until the algorithm balances the load and converges to the required standard deviation. Note that the algorithm tends to associate users to least loaded BSs. 


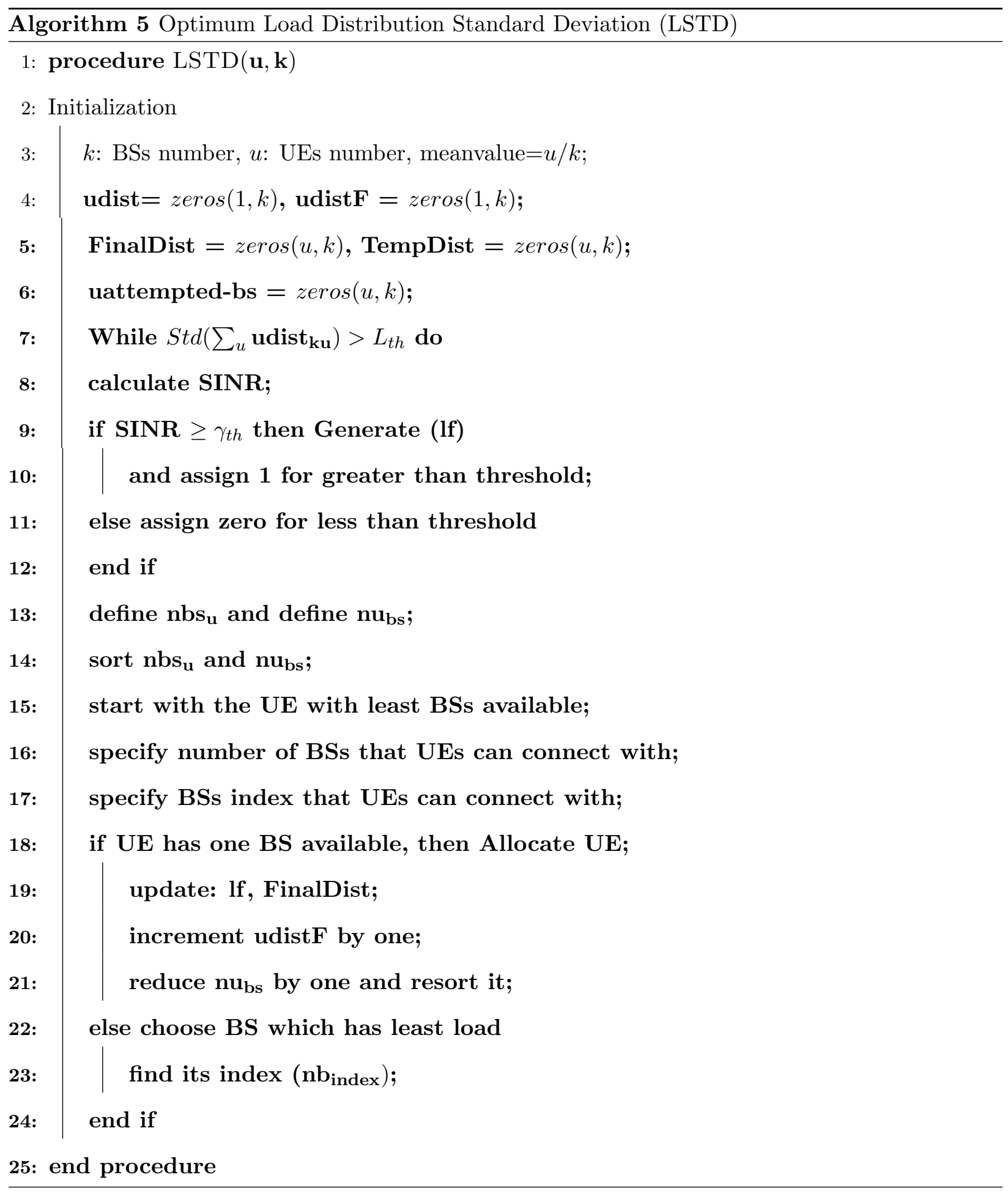




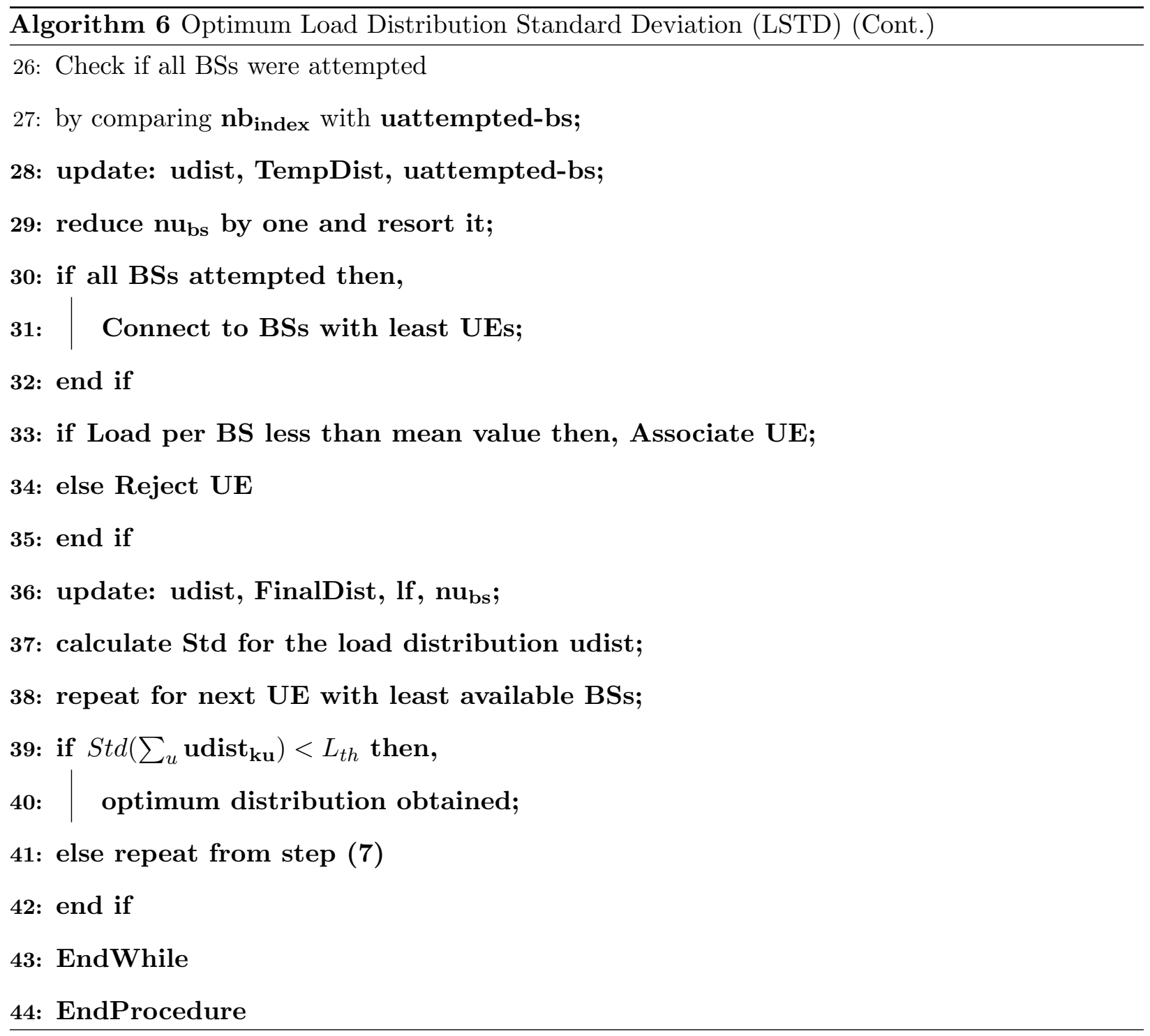




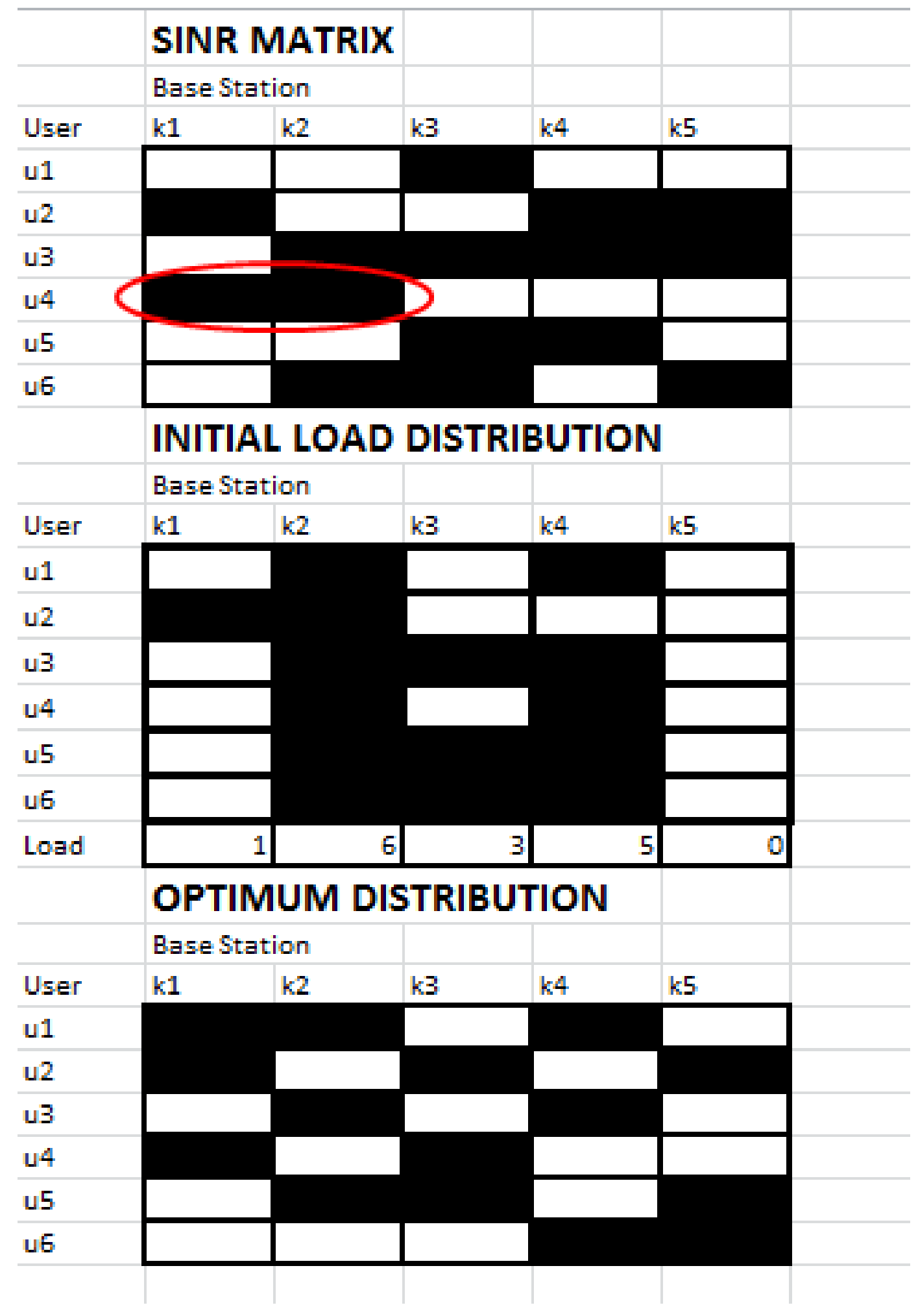

Figure 6.1: Proposed optimization for user distribution 


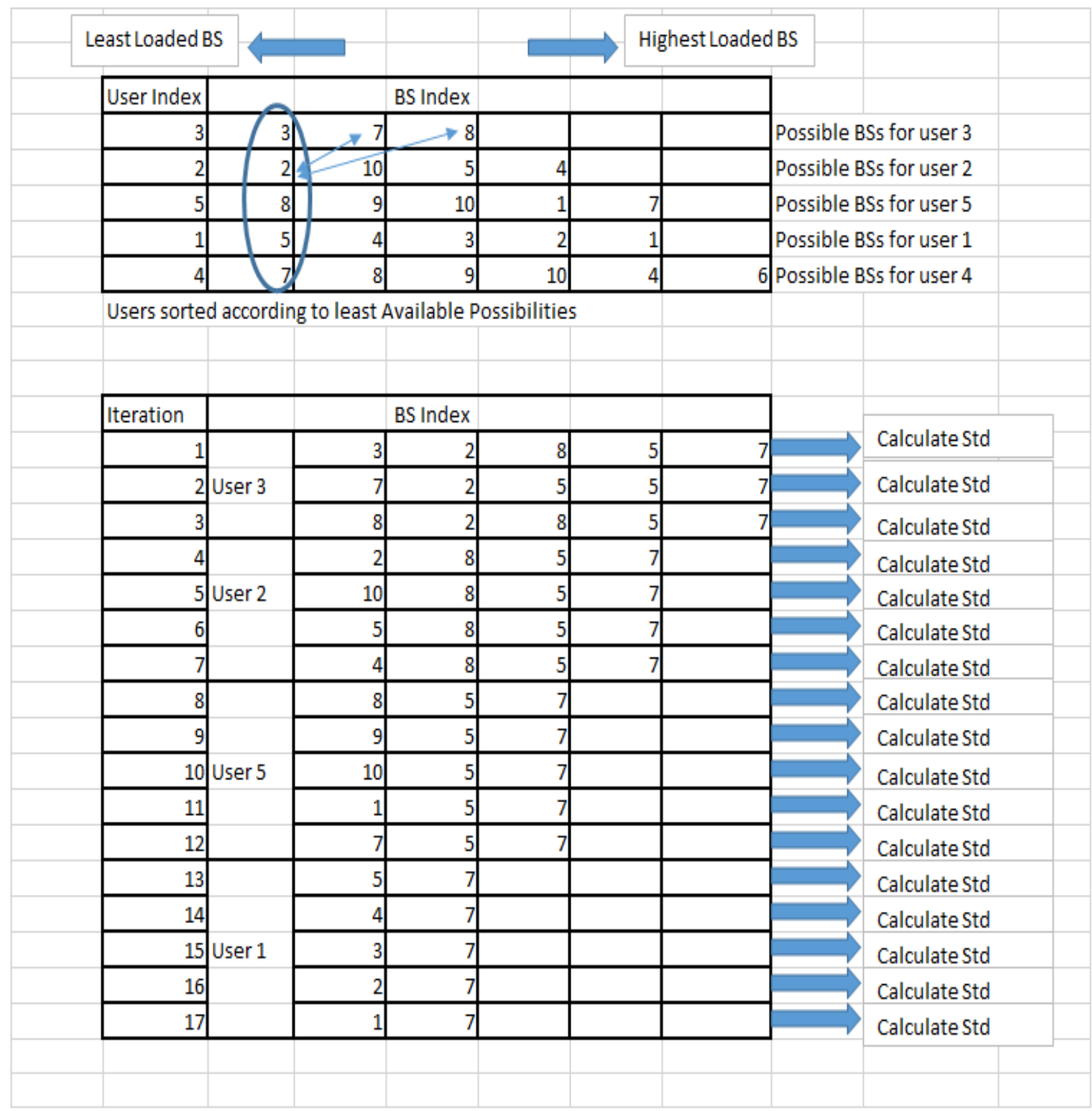

Figure 6.2: Explanation of the proposed user association algorithm selection criteria along iterations

As we described before that udist is a vector that presents network load or number of users in every BS, then our optimization problem is formulated as:

$$
\begin{array}{ll}
\min _{\text {udist }_{\mathbf{k u}}} & S t d\left(\sum_{u} \text { udist }_{\mathbf{k u}}\right) \\
\text { subject to } & C 1: S t d\left[\sum_{u} \text { udist }_{\mathbf{k u}}<L_{t h}\right] \\
& C 2: \gamma_{\mathbf{k u}}>\gamma_{t h} \\
& C 3: \sum_{u \in U} \mathbf{x}_{\mathbf{k u}}=1 ; k \in K \\
& C 4: \mathbf{u d i s t}_{\mathbf{k u}} \leq \mathbb{E}\left[L_{k}\right]
\end{array}
$$




\begin{tabular}{|c|c|c|c|c|c|c|c|c|c|c|c|c|c|c|c|}
\hline \multirow{3}{*}{$\begin{array}{l}\text { Total base } \\
\text { stations } \\
\text { available }\end{array}$} & \multirow{3}{*}{ user index } & nu= number of Users & 43 & 57 & 59 & 61 & 62 & 65 & 69 & 69 & 70 & 74 & 75 & 76 & 79 \\
\hline & & nbi = Base Station Number & 1 & 11 & 10 & 9 & 5 & 12 & 4 & 13 & 7 & 2 & 8 & 3 & 6 \\
\hline & & \multicolumn{3}{|c|}{ Istitteration } & & & & & \begin{tabular}{l|l} 
ui & p
\end{tabular} & & & & & & \\
\hline$n b$ & ui & 27 & 10 & 9 & 13 & 8 & & & 27] & 8 & 9) & 10 & 13 & & \\
\hline 4 & 27 & 43 & 11 & 5 & 4 & 1 & & & 43. & 4 & 5 & 7 & 11 & & \\
\hline 4 & 43 & 9 & 10 & 9 & 1 & 2 & 8 & & 9) & 2 & 7 & 8 & 9 & 10 & \\
\hline 5 & 9 & \multicolumn{3}{|c|}{ 2nd lteration } & & & & & & & & & & & \\
\hline 5 & 21 & 27 & 10 & 9 & 13 & 8 & & & & & & & & & \\
\hline 5 & 90 & 43 & 11 & 5 & 4 & 1 & & & & & & & & & \\
\hline 6 & 4 & 9 & 10 & 9 & 1 & 2 & 8 & & & & & & & & \\
\hline 6 & 6 & \multicolumn{3}{|c|}{ 3nd lteration } & & & & & & & & & & & \\
\hline 6 & 22 & 27 & 10 & 9 & 13 & 8 & & & & & & & & & \\
\hline 6 & 24 & 43 & 11 & 5 & 4 & 1 & & & & & & & & & \\
\hline 6 & 32 & 9 & 10 & 9 & 1 & 2 & 8 & & & & & & & & \\
\hline 6 & 37 & \multicolumn{3}{|c|}{ 4th literation } & & & & & & & & & & & \\
\hline 6 & 56 & 27 & 10 & 9 & 13 & 8 & & & & & & & & & \\
\hline 6 & 61 & 43 & 11 & 5 & 4 & 1 & & & & & & & & & \\
\hline 6 & 67 & 9 & 10 & 9 & 1 & 2 & 8 & & & & & & & & \\
\hline 6 & 79 & \multicolumn{3}{|c|}{ Sth literation } & & & & & & & & & & & \\
\hline 6 & 98 & 27) & 10 & 9 & 13 & 8 & & & & & & & & & \\
\hline 7 & 10 & 43 & 11 & 5 & 4 & 1 & & & & & & & & & \\
\hline 7 & 18 & 9 & 10 & 9 & 1 & 2 & 8 & & & & & & & & \\
\hline 1 & 25 & & & & & & & & & & & & & & \\
\hline 7 & 29 & & & & & & & & & & & & & & \\
\hline 7 & 31 & & & & & & & & & & & & & & \\
\hline 7 & 34 & & & & & & & & & & & & & & \\
\hline
\end{tabular}

Figure 6.3: Selection criteria from all available combinations

where constraint $C_{1}$ accounts that standard deviation of load distribution should be less than a certain threshold. Constraint $C_{2}$ depicts that SINR should be greater than a certain threshold. Constraint $C_{3}$ restricts that every UE can connect to only one BS at a time, and finally, $C_{4}$ employs that the final UE distribution per BS should not exceed the load mean value.

Our algorithm is explained as follows:

1. First, we calculate SINR matrix (lf) for every UE per BS and define all UE possibilities for connection based on a certain SINR threshold as shown in the upper part of Fig. 6.1.

2. We define $\mathbf{n b s}_{\mathbf{u}}$ (number of BSs per UE) by summing columns of (lf)and we define $\mathbf{n u}_{\mathbf{b s}}$ (number of 


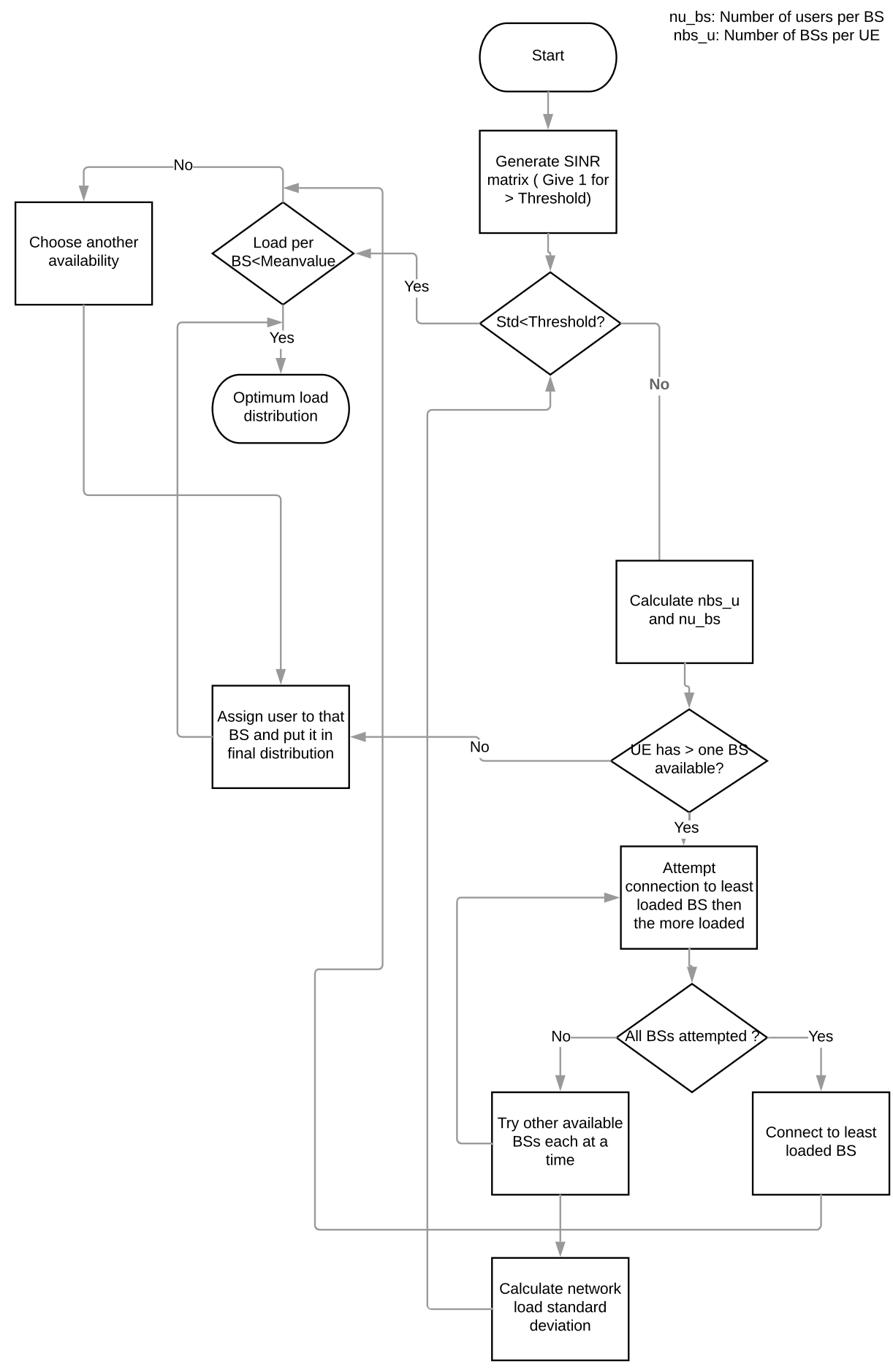

Figure 6.4: Flow chart of the proposed user association algorithm. 


\begin{tabular}{|c|c|c|c|c|c|c|c|c|c|c|c|}
\hline \multicolumn{11}{|c|}{ udist } & Std \\
\hline 88 & 86 & $y$ & 6 & P & J & 15 & 5 & 9 & 5 & 955 & 2.8245 \\
\hline 99 & 96 & 9 & 6 & 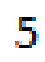 & 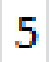 & 14 & 5 & 9 & 5 & 855 & 2.6561 \\
\hline 89 & 6 & 9 & 6 & 6 & 5 & 13 & 5 & 10 & 5 & 855 & 2.445 \\
\hline 89 & 6 & 8 & 6 & 6 & 6 & 12 & 5 & 10 & 5 & 955 & 2.2138 \\
\hline 89 & 96 & 8 & 6 & 6 & 6 & 12 & 6 & 9 & 5 & 955 & 2.0702 \\
\hline 88 & 36 & 8 & 6 & 6 & 6 & 11 & 6 & 9 & 6 & 956 & 1.7033 \\
\hline 88 & 37 & 8 & 6 & 6 & 6 & 11 & 6 & 8 & 6 & 866 & 1.4601 \\
\hline 88 & 87 & 8 & 7 & 6 & 6 & 10 & 6 & 8 & 6 & 866 & 1.2315 \\
\hline 88 & 37 & 8 & 7 & 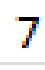 & 6 & 9 & 6 & 8 & 6 & 866 & 1.0271 \\
\hline 88 & 37 & $\varepsilon$ & & 7 & 0 & 8 & & 8 & & 86 & 644 \\
\hline
\end{tabular}

Figure 6.5: User distribution changing as standard deviation converges

UEs per BS) by summing rows of (lf) .

3. Then, we find the UE with least number of available BSs for connection and determine the BSs indices as $n b_{\text {index }}$. If only one BS is available, the UE has to connect with it. Whenever a UE is allocated it should be removed from the list. Next, allocate UE to one of the BSs which have less load (which has not been previously allocated) to generate the allocation distribution matrix according to the middle part of Fig. 6.1. After every BS is attempted for a certain UE, uattempted - bs has to be updated to indicate that BS has been already attempted. If no BSs are available, then choose the one with the least load.

4. Repeat for the next UE with least possible BSs available for connection and obtain UE distribution to BSs.

5. Sum the number of UEs associated to every BS and determine Standard deviation (Std) of UE-BS load distribution.

6. If Std is greater than a certain threshold $\left(L_{t h}\right)$, we start the second iteration. First, we try same UE with one of the other available BSs and recalculate Std. If value if still high, then we choose any one of the available BSs which has the highest SINR.

7. If load for the BS where UE is assigned is less than a mean value, then assign UE to it. Otherwise, we reject this UE. We meant by checking the mean value after calculating standard deviation is that if this base station reached the mean value (maximum load any BS can accept) or cannot go over this 


\begin{tabular}{|c|c|c|c|c|c|c|c|c|c|c|c|c|c|}
\hline \multicolumn{13}{|c|}{ udist } & \multirow{2}{*}{$\begin{array}{l}\text { std } \\
2.8245\end{array}$} \\
\hline 8 & 9 & 6 & 9 & 6 & 5 & 5 & 15 & 5 & 9 & 5 & 8 & 55 & \\
\hline 9 & 9 & 6 & 9 & 6 & 5 & 5 & 14 & 5 & 9 & 5 & 8 & 55 & 2.6561 \\
\hline 8 & 9 & 6 & 9 & 6 & 5 & 5 & 14 & 5 & 9 & 5 & 9 & 55 & 2.6561 \\
\hline 8 & 9 & 6 & 9 & 6 & 6 & 5 & 14 & 5 & 9 & 5 & 8 & 55 & 2.5678 \\
\hline 8 & 9 & 6 & 9 & 6 & 5 & 5 & 14 & 5 & 9 & 5 & 9 & 55 & 2.6561 \\
\hline 8 & 9 & 6 & 9 & 6 & 5 & 5 & 14 & 5 & 9 & 5 & 9 & 55 & 2.6561 \\
\hline 8 & 9 & 6 & 9 & 6 & 5 & 5 & 14 & 5 & 9 & 5 & 9 & 55 & 2.6561 \\
\hline 8 & 9 & 6 & 9 & 6 & 5 & 5 & 14 & 5 & 9 & 5 & 9 & 55 & 2.6561 \\
\hline 8 & 9 & 6 & 9 & 6 & 5 & 5 & 14 & 5 & 1 & 5 & 8 & 55 & 2.6849 \\
\hline 8 & 9 & 6 & 9 & 6 & 6 & 5 & 14 & 5 & 9 & 5 & 8 & 55 & 2.5678 \\
\hline 8 & 9 & 6 & 9 & 6 & 5 & 5 & 14 & 5 & 9 & 5 & 9 & 55 & 2.6561 \\
\hline 8 & 9 & 6 & 9 & 6 & 6 & 5 & 14 & 5 & 9 & 5 & 8 & 55 & 2.5678 \\
\hline 8 & 9 & 6 & 9 & 6 & 5 & 5 & 14 & 5 & 1 & 5 & & 55 & 2.6849 \\
\hline 8 & 9 & 6 & 9 & 6 & 6 & 5 & 14 & 5 & 9 & 5 & 8 & 55 & 2.5678 \\
\hline 8 & 9 & 6 & 9 & 6 & 5 & 5 & 14 & 5 & 9 & 5 & 9 & 55 & 2.6561 \\
\hline 8 & 9 & 6 & 9 & 6 & 6 & 5 & 14 & 5 & 9 & 5 & 8 & 55 & 2.5678 \\
\hline 8 & 9 & 6 & 9 & 6 & 6 & 5 & 13 & 5 & 1 & 5 & & 55 & 2.445 \\
\hline
\end{tabular}

Figure 6.6: Transitional iterations as load in one BS changes

value then if user has other options for connection then it should go to them and leave this fully loaded base station that reached mean value. If we do not have other options available then it will sadly connect to that base station despite it is saturated and already reached mean value.(meaning that if best option or best base station is already fully loaded then user should choose another base station from the available base stations)

8. Repeat the same process for the rest of the UEs until Std value falls below the threshold.

9. Obtain the optimum load distribution matrix as shown in the lower part of Fig. 6.1.

10. Finally, the number of UEs associated per BS should be equal or nearly equal for all BSs.

\subsection{Simulation Analysis}

Matlab $^{T M}$ simulation was used to analyze the performance of the LSTD UE association algorithm. We evaluate the efficacy of the proposed methods in comparison with the conventional max-SINR algorithm. 
Table 6.1 lists the values we used in estimating the performance of the proposed method.

In Fig. 6.7, we plot the load distribution for our suggested algorithm and compare it with the classic max-SINR UE association algorithm for 100 UEs and 12 BSs. As we see, the conventional UE association method has the shortcoming of an unbalanced load distribution. Whereas, optimizing the load distribution yields a fair distribution of load among all nodes.

Fig. 6.8 shows a comparison of the amount of consumed bandwidth from the total bandwidth in the conventional UE association method and our suggested method. It is clearly seen that in the classic UE association algorithm, some BSs are suffering from bandwidth shortage as they are unable to provide adequate service for all associated UEs. Link failure and poor service quality will result as a consequence of inability of BSs to serve all their associated UEs.

Fig. 6.9 presents the load standard deviation for a number of UEs, which represents our objective function as it converges with iterations. Here, as the number of UEs in the network increases, the load standard deviation value starts at a higher level and requires more time and more iterations to converge. Fig. 6.10 shows a comparison of the objective function as it converges for the cases of high and low Macro BS powers. Higher power is associated longer time and more iterations to reach optimum goal.

\subsection{Chapter Summary}

HetNets are gaining the attraction of the communication society as they work in multi-layers cooperating together to fulfill the dream of connecting the globe as one big network in the future. In this chapter, we introduced a novel algorithm for allocating UEs to the corresponding BSs based on optimizing the load standard deviation in an OFCDM system with spreading in time and frequency domains. Our goal was to

Table 6.1: Parameters used for LSTD user association algorithm

\begin{tabular}{|c|c|}
\hline Parameter & Value \\
\hline Intensity of Initial Micro BSs Positions & 20 \\
\hline Mean Number of UEs of each BS $\left(\lambda_{u}\right)$ & 200 \\
\hline Micro Cell Radius & $500(\mathrm{~m})$ \\
\hline Min Allowed Distance Between Micro BSs & $200(\mathrm{~m})$ \\
\hline Macro BS Transmitted Power & $50($ Watt $)$ \\
\hline Noise Power & $20($ Watt $)$ \\
\hline SINR Threshold & 0.5 \\
\hline Standard Deviation Threshold & 1 \\
\hline
\end{tabular}




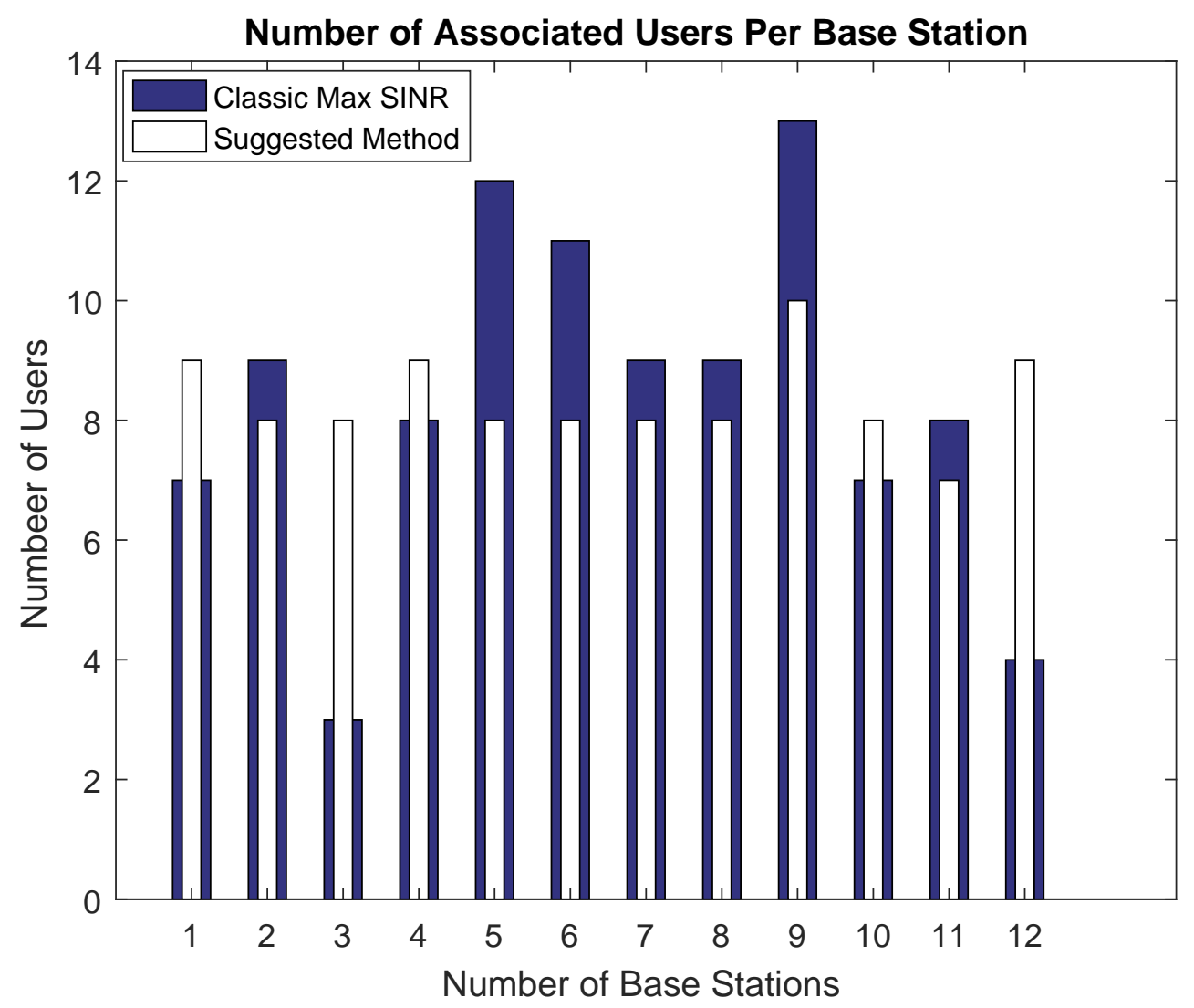

Figure 6.7: Comparison between network load of the proposed optimum standard deviation user association versus Max-SINR

reach a nearly linear standard deviation curve so that UE load is nearly uniform. We started initially from an acceptable SINR level to ensure that even when UEs are associated to another BS, they still are getting a strong signal and the link does not fail. Our algorithm is considering both; load and SINR level. Our results provide excellent improvement in system performance. 


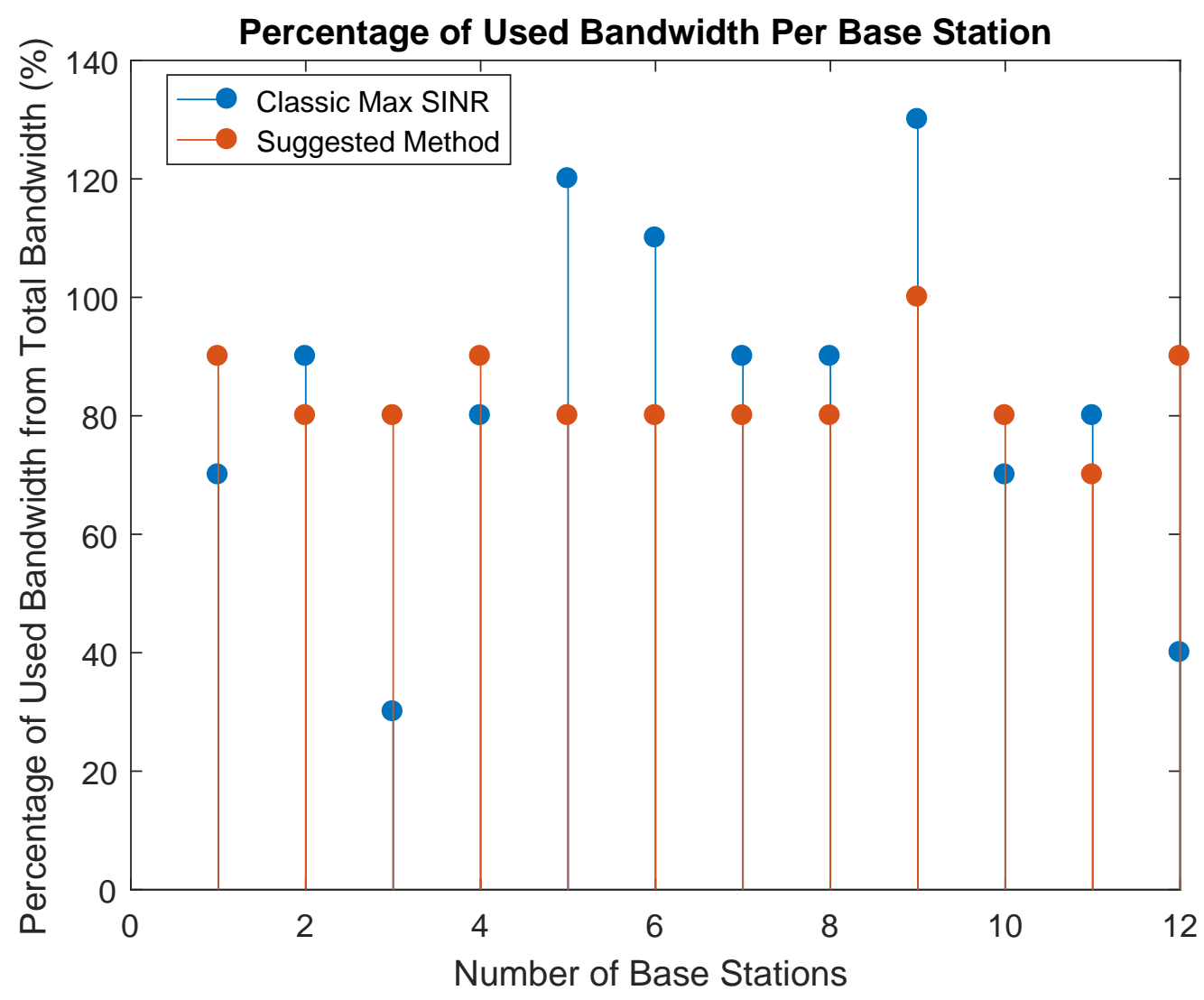

Figure 6.8: Comparison between the percentage of used bandwidth of the proposed standard deviation user association versus Max-SINR 


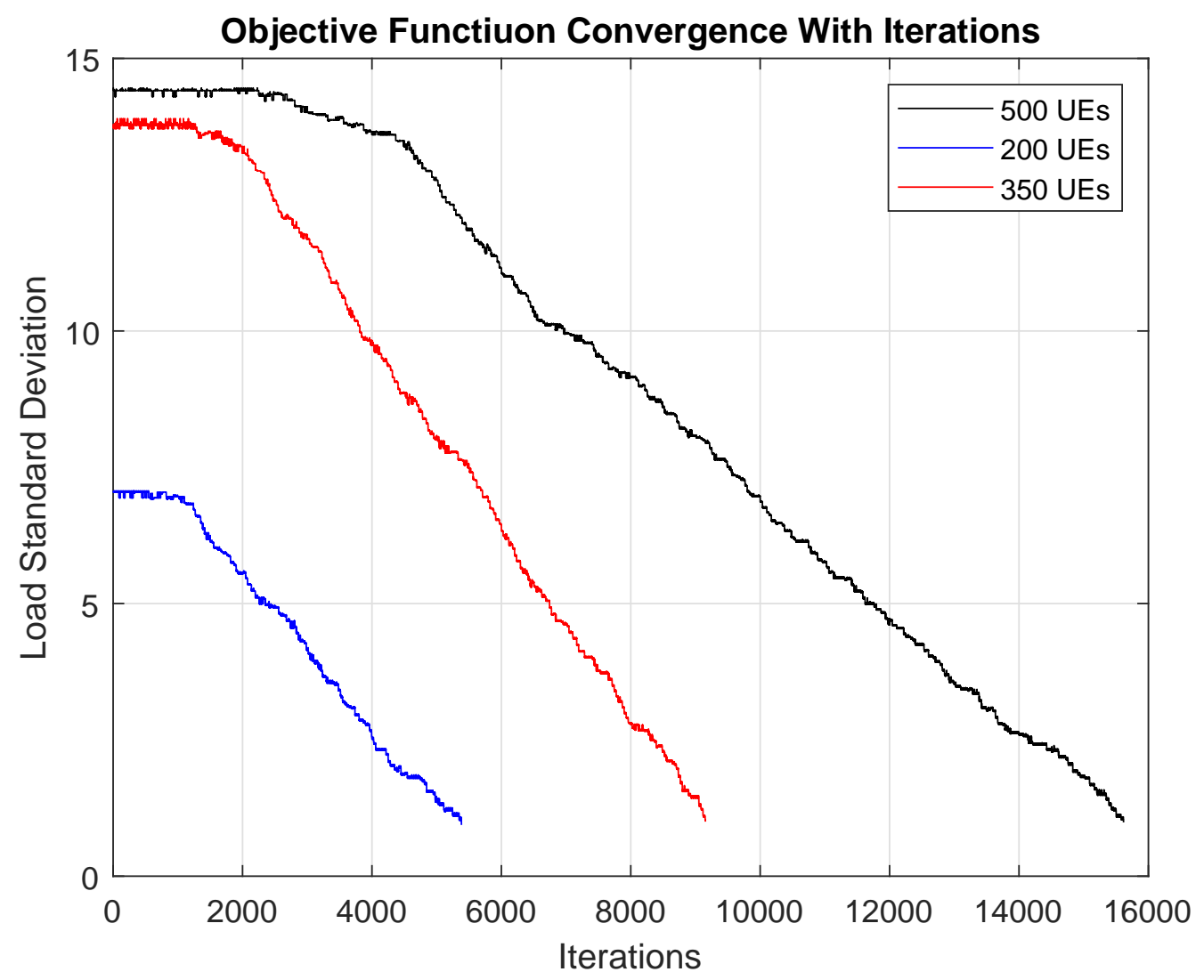

Figure 6.9: Comparison between network load standard deviation for various UE numbers 


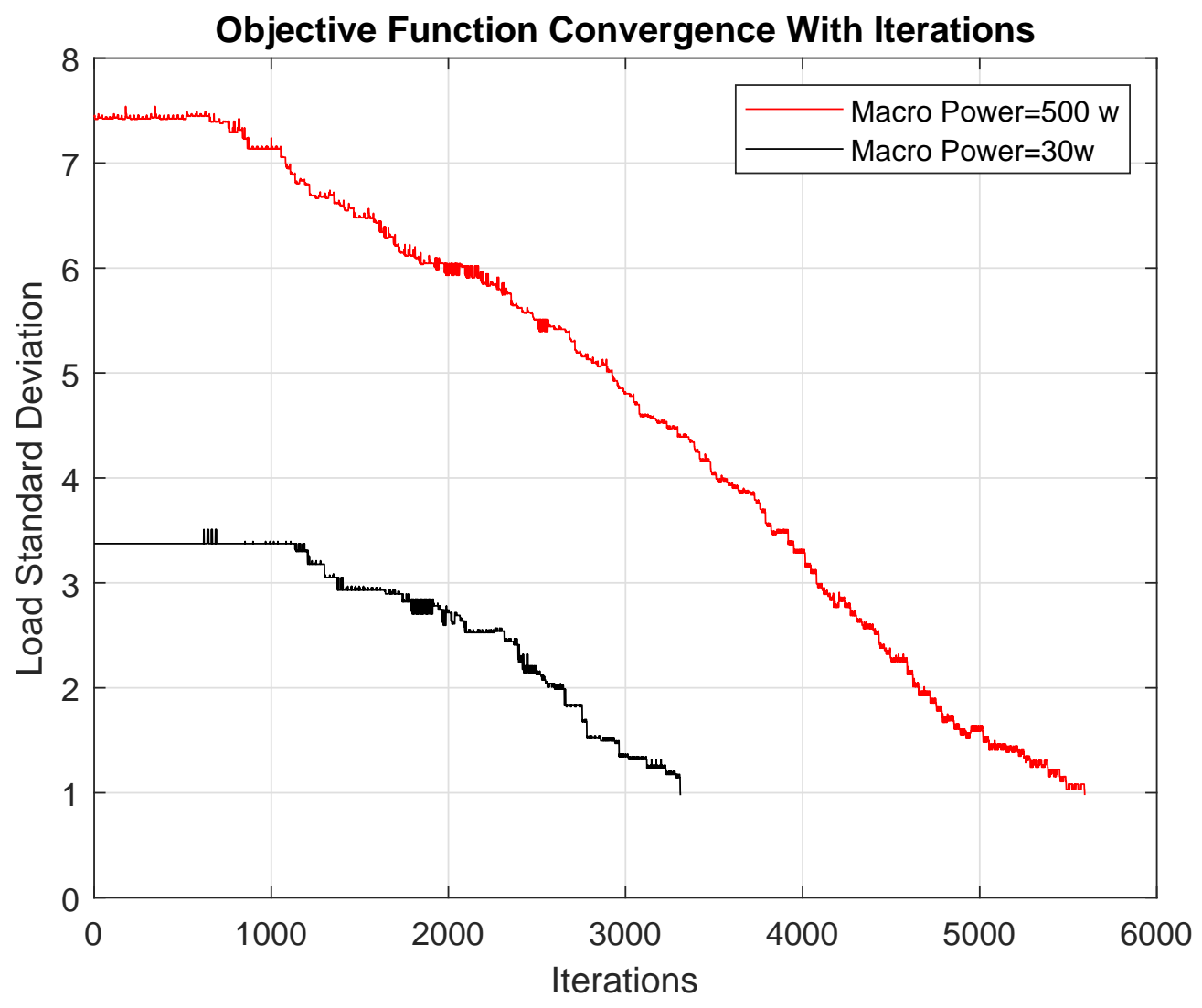

Figure 6.10: Comparison between network load standard deviation for various Macro BS powers 
CHAPTER 7

\section{Real Time Load Balance (RTLB) UE Association}

\section{Algorithm}

\section{$7.1 \quad$ Introduction}

The research on UE association in HetNBets is limited and is still in its first steps despite its significant effect on the performance of HetNets.

The classic max-SINR or max-RSS user association algorithms (where the UE connects to the BS with the highest received signal strength or the highest signal-to-interference-and noise ratio) are inappropriate solutions for HetNets due to their nature of variable transmit BS antennas power levels. The aforementioned methods tend to associate UEs to the BSs with the best signal quality or strength; however, they are not best suited for HetNets as UEs in this context will tend to connect to the Macro BS, which is the one with the highest signal power. A severe load imbalance and significant inefficiency arises and impacts the performance.

Reassociation/handover of UEs upon data set change, i.e.: when new UEs are added or dropped or when UEs move from one location to another is very challenging and requires a fast efficient algorithm to cope with the rapid changes.

\subsection{Related Work}

Most of the work that has been done so far to allocate UEs to BSs in multi-tier networks focused on allocating UEs to BSs, which are either having the maximum received signal power, maximum SINR, sum rate [178], weighted sum energy efficiency [177], or per-user utility function [179]. We believe that all the aforementioned 
algorithms are not better than maximizing SINR, where they either tend to allocate UEs to Macro BSs that have the strongest transmitted signal power, or tend to associate UEs to small cell BSs leaving Macro BSs with less load.

To overcome the drawbacks of max-SINR and max-RSS in HetNets, some efforts introduced SINR-biased user association algorithm [182]. Signal strength of Micro tier BSs is artificially enhanced by multiplying it by a certain bias to increase number of UEs associated to Micro BSs. A significant increase in system throughput and capacity is noted; however biased Micro BSs performance was degraded by strong interference from the Macro BS. Some research efforts [183-186] focused on optimizing the value of the bias to maximize network utility, throughput, and reduce outage probability. Also, [187] introduced the rate-biased user association algorithm as another solution to overcome the drawbacks of max-SINR user association. This algorithm tended to reduce the load of Macro BSs and offer more load to Micro BSs.

In [188], the authors employed the Selective Small Cell BS Deployment (SBS) as a method of UE association, where the area around the Macro cell is divided into center and edge regions. Micro BSs are only active in the region where Macro coverage is poor). This approach is commendable, but may have some practical limitations. In [189], the authors combined UE association algorithms using SBS along with traffic offloading using Cell Range Expansion (CRE). SINR distribution analysis, coverage probability, rate coverage, and deduced semiclosed-form expressions metrics. Similarly as in [188], the working region is divided into center and edge regions. The drawback of CRE is that the Macro BS now acts as a strong interferer and SINR level calculated at it is reduced.

Also, some research was directed to introduce new interference mitigation techniques designed specifically for the biased HetNet Scenarios to overcome interference from Macro BSs like the Inter Cell Interference Coordination (ICIC) and the enhanced version Enhanced Inter Cell Interference Coordination (eICIC) techniques. In [190], the authors introduced the dynamic eICIC considering different mobility scenarios, traffic mixes, and adaptation rate to achieve various network objectives. Also, authors in [183] introduced a joint optimization of Almost Blank Subframes (ABS) and UE-association based on real network data to determine the amount of radio resources assigned to Pico cells and to establish the association rules for UE selection. In [191], the authors studied the joint problem of UE association and spectrum allocation in multi-tier HetNets. The UE connects to a BS based on the maximum biased downlink received power. After that, the authors optimized network utility, optimal spectrum partition, and optimum user association. The joint problem of user association and ABS ratio issue with eICIC scheme in HetNets is studied in [192]. A user association algorithm with polynomial complexity is proposed and system throughput and users service fairness are analyzed through simulation results. Furthermore, in [193] the authors propose an optimization algorithm with multiple coexisting network services (unicast, multicast and VO services) to maximize user utility. The proposed eICIC configuration has three layers (service layer, BS layer, and network layer. The 
results suggested that the proposed algorithm outperforms other solutions. Also, in [194] the authors introduce the joint optimization problem of load balancing of downlink and uplink to maximize the total utility for UE rate in eICIC HetNets. In addition, the authors proposed a method based on configuring uplink transmission in the Macrocell in ABSs (UMABS) to improve the efficiency of the ABS, balance the load, and improve system capacity users rate.

\subsection{Contributions and Organization}

In this chapter, we introduce a new dynamic user association algorithm in cooperating open access HetNets. Our algorithm works very fast and benefits from the ideas that BSs can share their load information and status (accepting or not accepting UEs at this moment) with each other and that traffic transfer is possible among BSs. We use real-time scenarios, where users motility and status change are considered. Users motion leads to changing the network data set at every instance and users' status change means that some users can be added or dropped at a given time instant. We associate UEs initially based on maximum SINR, then select the UE having highest SINR value to ensure that a strong enough SINR level is met.

The rest of this chapter is organized as follows: Section 7.4 presents the description and explanation of RTLB user association algorithm. Section 7.5 presents the problem formulation. In Section 7.6, we present mathematical analysis for UEs' mobility and status change. Section 7.7 presents overage probability mathematical analysis fore mobile UEs. Section 7.8 shows the results of both algorithms and Section 7.9 concludes this chapter.

\subsection{Explanation of RTLB User Association Algorithm}

We propose a dynamic distributed UE association algorithms for cooperating BSs in HetNets. We assume BSs share their load information among each other and that traffic transfer occurs from lightly loaded BSs and vice versa when BSs broadcast their status to other BSs in every iteration or data set change. Our algorithm is fast and effective in redeployment problem in rapidly changing network scenarios when UEs positions change or when new UEs are added or dropped from the network. We only process the change that happens in evey iteration and we do not start the algorithm all over from the beginning. To implement this algorithm, initial SINR from all UEs to each BS is required, in order to decide UEs that will be associated to every BS based on maximum SINR value it is getting from which BS. Also, load for every BS has to be well known in every iteration to specify every BS status. Fig. 7.1 represents an explanation of how our proposed algorithm selects the UE to be removed off the over loaded BS. All UEs in the network (whether they are active or inactive) are assigned a unique Identification Number (ID). After specifying the giving BSs and 


\begin{tabular}{|c|c|c|c|c|c|c|c|c|c|}
\hline udist2 & 8 & 6 & 4 & 4 & 10 & 3 & & & \\
\hline \multirow[t]{10}{*}{ bs_uid } & 5 & 75 & 18 & 23 & 480 & 55 & & & \\
\hline & 253 & 8 & 150 & 13 & 7 & 7 & UE 13 & 3 & 4 \\
\hline & 45 & 58 & 13 & 150 & 27 & 458 & SINR & 0.86 & 0.93 \\
\hline & 20 & 253 & 458 & 253 & 65 & & & & \\
\hline & 13 & 369 & & & 45 & & & & \\
\hline & 150 & 60 & & & 17 & & & & \\
\hline & 458 & & & & 23 & & & & \\
\hline & 89 & & & & 18 & & & & \\
\hline & & & & & 55 & & & & \\
\hline & & & & & 88 & & & & \\
\hline \multicolumn{10}{|l|}{ Threshold $=6$} \\
\hline BS STATUS & -1 & 0 & 1 & 1 & -1 & 1 & & & \\
\hline & & & & & & & & & \\
\hline 0.9649 & 13 & & & & & & & & \\
\hline 0.9575 & 458 & & & & & & & & \\
\hline 0.89 & 150 & & & & & & & & \\
\hline 0.654 & 89 & & & & & & & & \\
\hline 0.5469 & 45 & & & & & & & & \\
\hline 0.35 & 20 & & & & & & & & \\
\hline 0.0975 & 253 & & & & & & & & \\
\hline udist2 & 7 & 6 & 3 & 4 & 10 & 3 & & & \\
\hline BS STATUS & -1 & 0 & 1 & 1 & -1 & 1 & & & \\
\hline
\end{tabular}

Figure 7.1: Selection criteria for UEs at every giving BS and for the accepting BS

starting from the first giving BS, we specify the IDs of UEs associated with the first giving BS and move the one with highest SINR value to the accepting BS with highest SINR value. We repeat for all extra UEs associated with this BS then for the rest of the giving BSs. 


\subsection{Problem Formulation and Complexity Analysis}

We formulated our optimization problem as follows:

$$
\begin{array}{ll}
\max _{\text {udist }_{\mathbf{k u}}} & \mathbf{S I N R}_{\mathbf{k u}} \\
\text { subject to } & C 1: \max \left(\operatorname{SINR}\left(\text { bs }_{\text {uid }}(k)\right)\right)=u_{\text {max }} ; \forall \text { StatusBaseStation }(k)=-1 \\
& C 2: \operatorname{SINR}\left(\text { bsids }\left(u_{\text {max }}\right)\right)=\max ; \forall \text { StatusBaseStation }(k)=1 \\
& C 3: \sum_{u \in U} \mathbf{x}_{\mathbf{k u}}=1 ; k \in K \\
& C 4: \text { udist }_{\mathbf{k u}} \leq \mathbb{E}\left[L_{k}\right]+\text { margin }
\end{array}
$$

where constraint $C_{1}$ accounts that the user which has the max SINR (umax) for the giving BS should be selected first. Constraint $C_{2}$ depicts that the selected user previously should move to the accepting BS having the maximum SINR value as long as it is one of the possibilities for this user. Constraint $C_{3}$ restricts that every UE can connect to only one BS at a time, and finally, $C_{4}$ implies that the final UE distribution per BS should not exceed the load mean value plus a margin to allow some capacity for exceeding the mean value as the user might not have any other option.

The flow chart for the first iteration (without motion or user status change) of our proposed algorithm is shown in Fig. 7.2.

Our algorithm is explained in blocks Algorithm 7 and Algorithm 8. The details are as follows:

1. Generate initial locations of Micro BSs PPP.

2. Generate $N_{t}$ total number of UEs in the working area, and distribute them uniformly.

3. From the total $N_{t}$ UEs, generate a number $N_{a}$ of active UEs. Note that in the first iteration, UEs have not been added or dropped or changed position.

4. We divide the working area into $G$ regions and specify locations of active UEs.

5. As some UEs are mobile, fading gain matrix $h$ for every active UE will be attributed to location. Every location inside a region in the network area has a specific $h$ value. Note that every location is viewed differently at each BS, so every location is a vector equal to the number of Micro BSs.

6. Calculate distances from all active UEs to all BSs and calculate signal power matrix from all BSs to every active UE. Also, calculate interference matrix from adjacent BSs to each UE.

7. Consider SINR, and values above a certain SINR threshold will get a value of 1 , otherwise 0 .

8. Consider a certain load threshold (LoadThreshold) for every BS. Generate a flag for every BS status, and BSs will broadcast their status information among other BSs (StatusBaseStation) according to the following: 


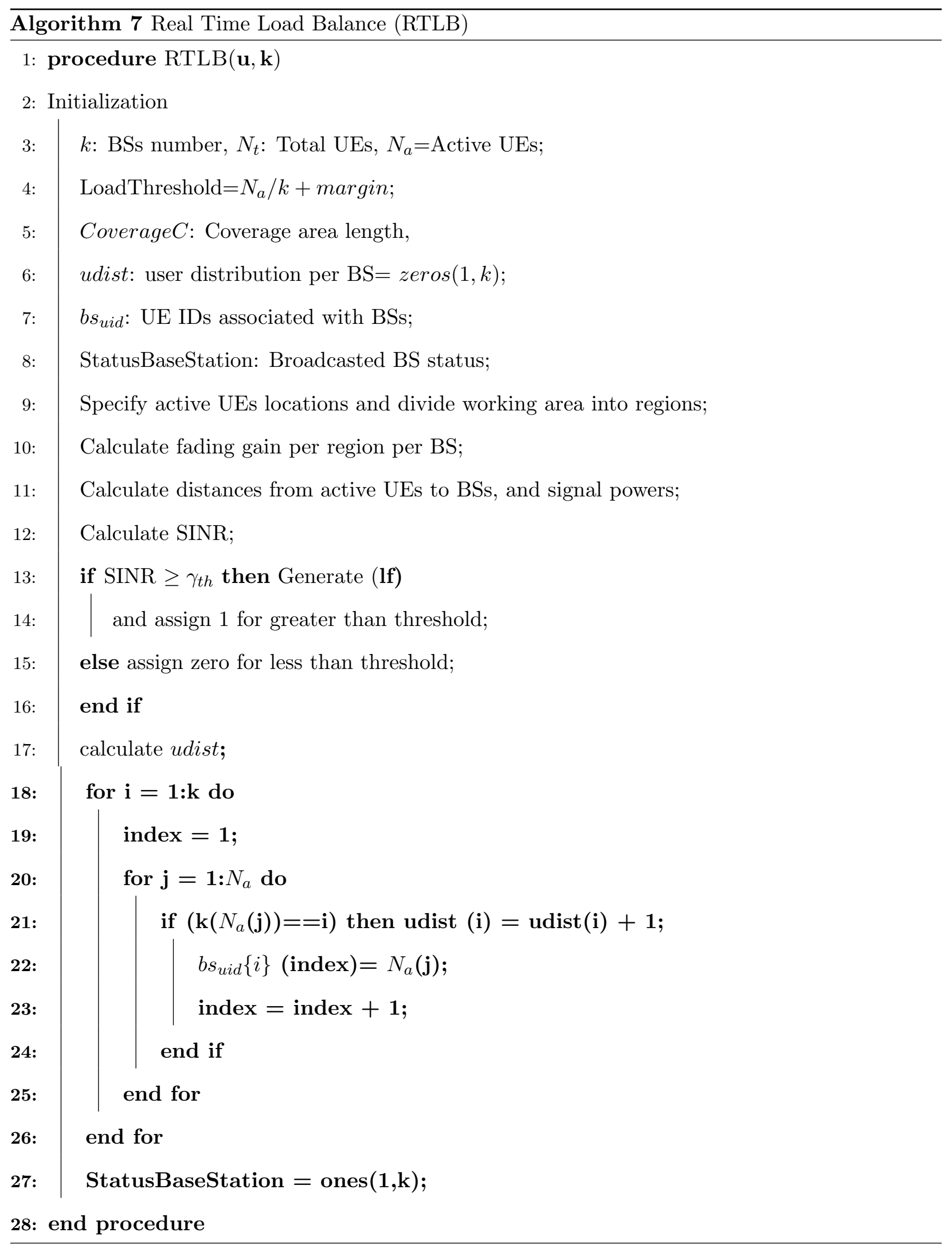




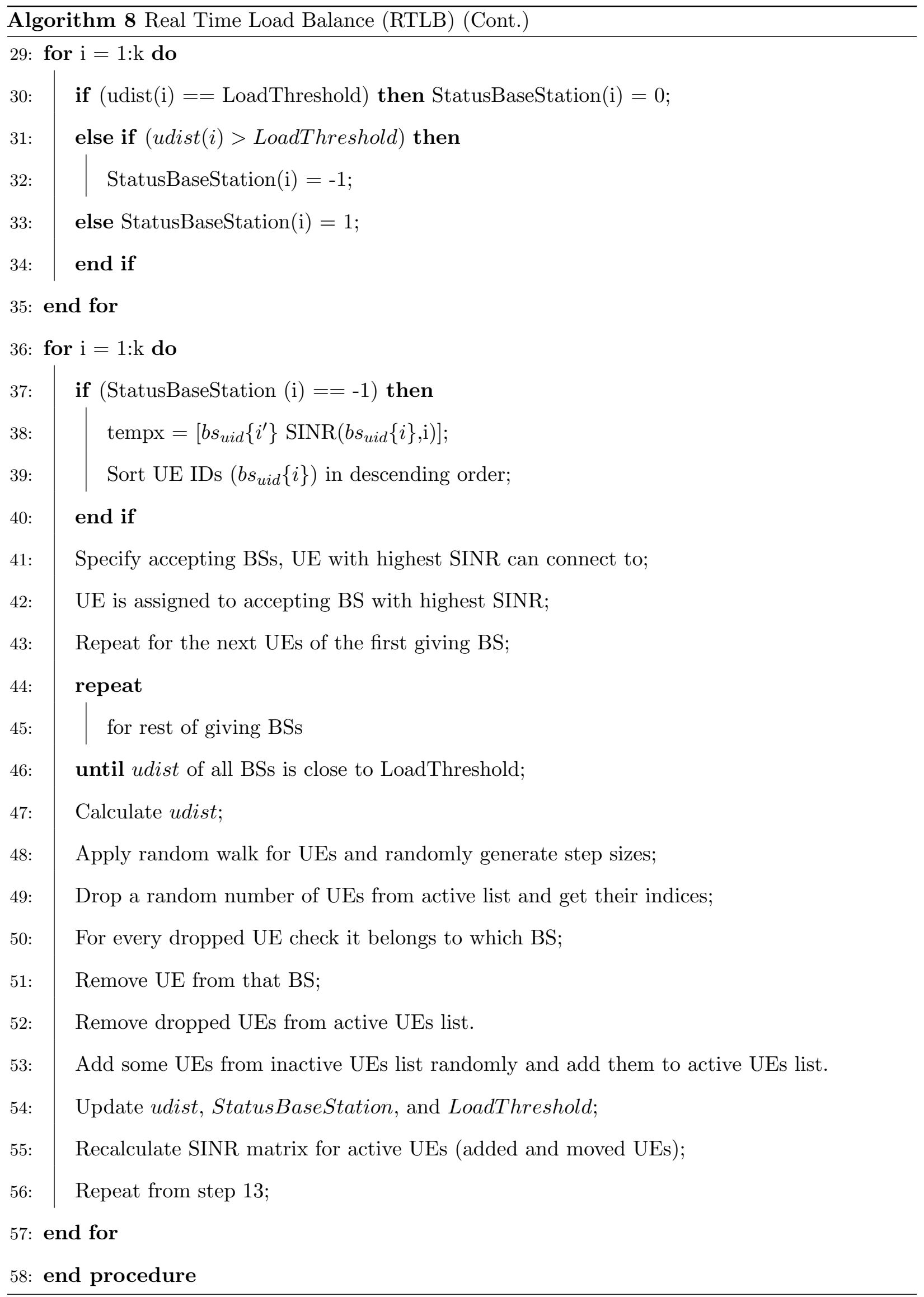




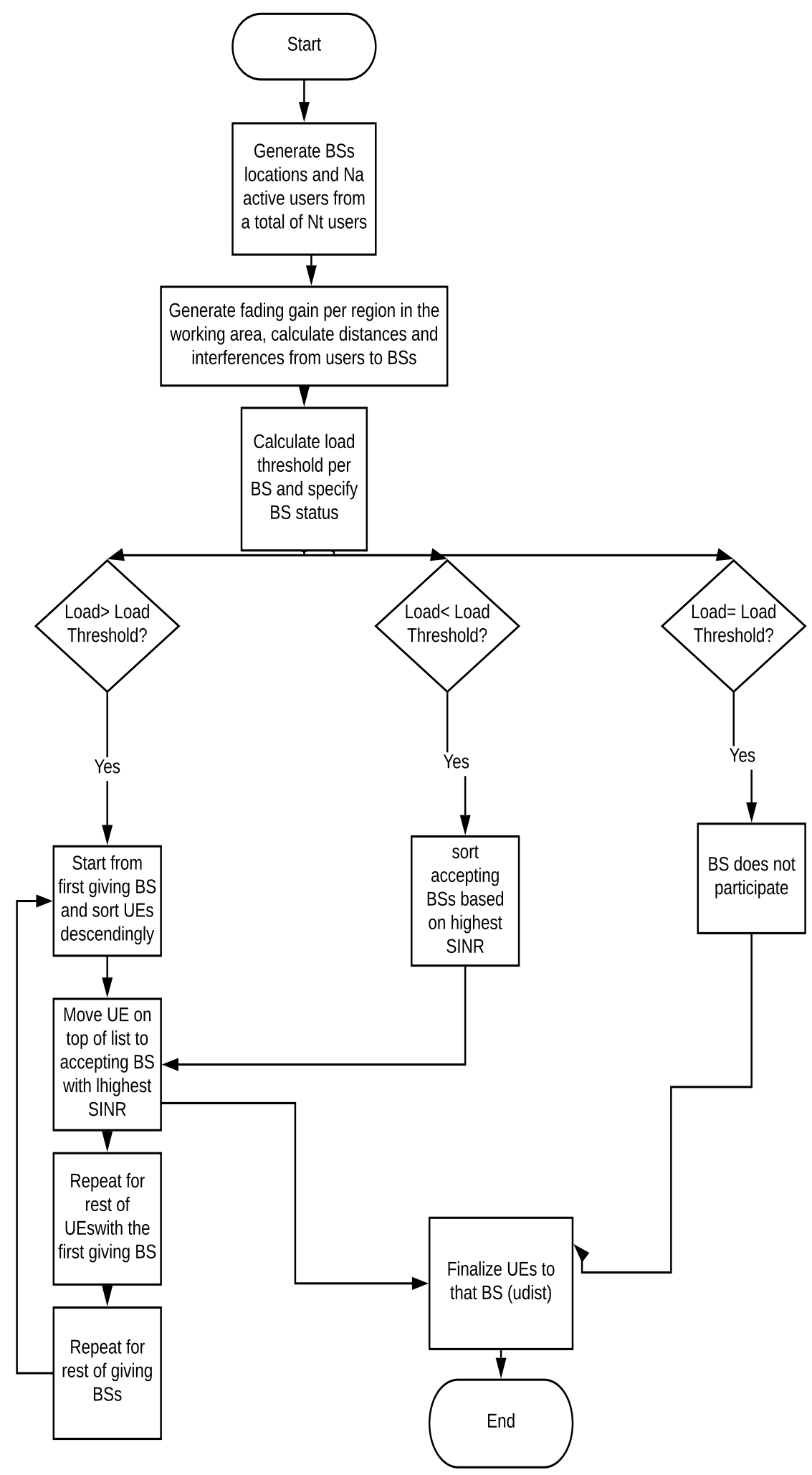

Figure 7.2: Flow chart for the first iteration of our proposed RTLB user association algorithm. 


$$
\begin{cases}-1, & \text { Giving BSs with more UEs than load threshold. } \\ 1, & \text { Taking BSs with less UEs than load threshold. } \\ 0, & \text { Fully loaded BSs with the exact value of the load threshold. }\end{cases}
$$

9. Specify which BSs are in giving state. Start from first giving BS and specify indices of UEs associated with it as $b s_{\text {uid }}$.

10. Sort UEs in descending order of their SINR values. The UE with highest SINR value should go first to prevent the drastic drop in SINR when moving to another BS and denote this UE as $\left(u_{\max }\right)$.

11. Identify BSs that can accept the given UE, sort their indices in descending order as (bsids), and move UE to the one having the highest SINR value. If UE cannot go to any other BS, it will stay with its current BS. Repeat for the rest of extra UEs with the first giving BS.

12. Remove UE from the giving BS list to the accepting BS list and update BSs statuses.

13. Repeat for the other giving BSs and continue until most BSs will reach the load threshold value.

14. Second iteration will start when UEs status has changed (UE has been added or dropped) or UE has moved to a new location.

15. We have a new set of active UEs in every iteration when UEs are randomly added ( new UEs are added from total $N_{t}$ UEs or dropped (removed from the active UEs list).

16. Dropped UEs are removed from active UEs list to inactive UEs list, and update udist, BS status, and check load threshold.

17. Newly added UEs are removed from the inactive list to the active list.

18. Calculate interference, power, and SINR matrices for added UEs only and allocate them to accepting BSs one by one and update BS status and load threshold.

19. In case no BS can take a specific UE, we allocate it to the BS with highest SINR.

20. We assume that some UEs will move according to a random walk within the working area and we generate new locations of UEs.

21. Update the active UEs list, calculate SINR matrix for active UEs only, and repeat the same process of finding the giving BSs. 


\subsection{UEs Mobility and Status Change Analysis}

We consider that the total number of UEs in the system is $N_{t}$, where only $N_{a}$ are active at a given instant. Some UEs are moving in a random walk, where $N_{m}$ represents the number of active mobile UEs. $N_{D}$ UEs may be dropped at any instant due to call service failure, and $N_{d}$ UEs may replace them. Also, $\lambda_{N_{a}}$ is the Poisson arrival rate of active UEs, $\lambda_{N_{m}}$ is the Poisson arrival rate of active mobile UEs.

From the Poisson point process, the number of active users follows a Poisson distribution, hence, the probability density function of a random variable $N_{a}$, where $N_{a}$ are active UEs (whether they are mobile or not) is:

$$
f_{N_{a}}\left(N_{a}\right)=\exp ^{-\lambda_{N_{a}}} \frac{\lambda_{N_{a}}^{N_{a}}}{N_{a} !}
$$

The conditional probability (follows from the conditional probability of a Poisson distribution)that UEs from the active set turn out to be mobile or in other words the Probability of having an active UE served by a BS whether it is mobile or not (conditional distribution of $N_{m}$ given $N_{a}$ ) is:

$$
f_{N_{m} \mid N_{a}}\left(N_{m} \mid N_{a}\right)=\exp ^{-\lambda_{N_{m}}} \frac{\lambda_{N_{m}}^{N_{a}}}{N_{a} !}
$$

The probability that there are $N_{m}$ active mobile UEs is:

$$
f_{N_{m}}\left(N_{m}\right)=\sum_{N_{a}=1}^{N_{t}} f_{N_{a}}\left(N_{a}\right) \cdot f_{N_{m} / N_{a}}\left(N_{m} / N_{a}\right)
$$

Substituting by (7.2) and (7.3) into (7.4), we get:

$$
f_{N_{m}}\left(N_{m}\right)=\sum_{N_{a}=1}^{N_{t}} \exp ^{-\lambda_{N_{a}}} \frac{\lambda_{N_{m}}^{N_{a}}}{N_{a} !} \exp ^{-\lambda_{N_{m}}} \frac{\lambda_{N_{m}}^{N_{a}}}{N_{a} !}
$$

From the convergence of the series:

$$
f_{N_{m}}\left(N_{m}\right)=\exp ^{-\left(\lambda_{N_{a}}+\lambda_{N_{m}}\right)}\left|\frac{\lambda_{N_{a}} \lambda_{N_{m}}}{\left(N_{a}+1\right)^{2}}\right|
$$

Probability that there are $N_{D}$ UEs dropped from the active UEs set is $\frac{N_{D}}{N_{a}}$, and probability that there are $N_{d}$ UEs added is $\frac{N_{d}}{\left(N_{t}-N_{a}\right)}$

Probability that all active mobile UEs will be dropped at least once in the interval $t=T$ is:

$$
P_{D}=\frac{N_{D}}{N_{a}} N_{D}\left(\lambda_{d} t\right) \exp ^{-\lambda_{d} t} f_{N_{m}}\left(N_{m}\right)
$$

where $\lambda_{d}=\frac{N_{D}}{N_{d}}$ is the call drop rate. 


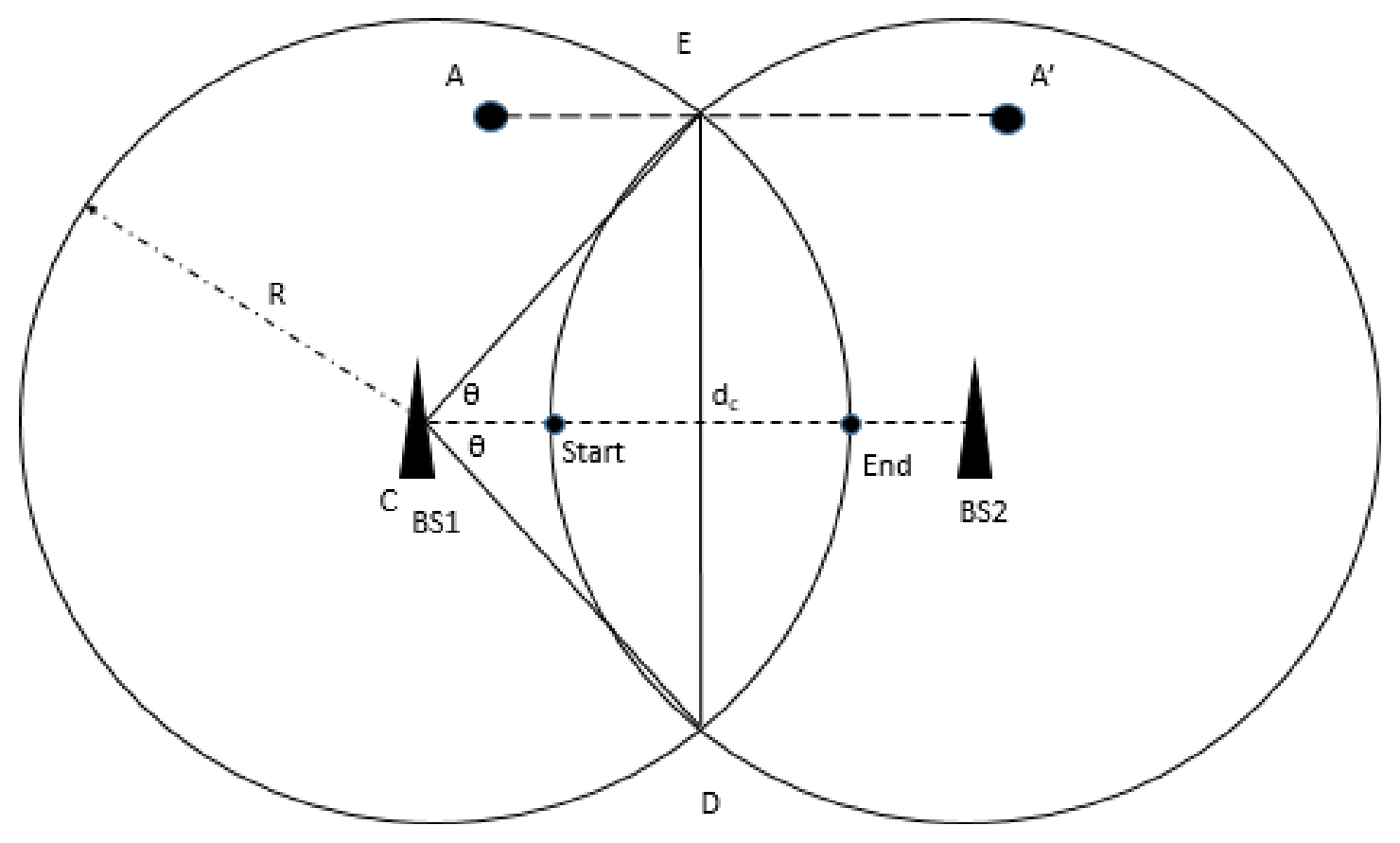

Figure 7.3: An illustration of handover from one cell to another.

\subsection{Coverage Probability Considering Moving Users}

SINR for the downlink of a given BS $k$ to its associated UE $u$ on subcarrier $z$ in an OFCDM channel will be:

$$
\gamma_{\mathbf{k}}^{(\mathbf{z})}\left(\mathbf{r}_{\mathbf{k}}\right)=\frac{N^{2} E_{c}^{2} \lambda_{k} P_{T_{k}}\left\|\mathbf{r}_{\mathbf{k}}\right\|^{-\alpha} \sum_{z \in G_{d}}\left(\mathbf{h}_{\mathbf{k}}{ }^{(z)}\right)}{\sum_{q=1, q \neq k}^{k} N^{2} E_{c}^{2} P_{T_{q}}\left\|\mathbf{r}_{\mathbf{q}}\right\|^{-\alpha} \sum_{z \in G_{d}}\left(\mathbf{h}_{\mathbf{q}}{ }^{(z)}\right)+\sigma_{n}^{2}}
$$

where, $N^{2} E_{c}^{2}$ is the power of the PN sequence used for spreading the signal, $\left\|r_{k}\right\|$ is the distance from the BS to UE $u, \mathbf{h}_{\mathbf{k}}$ is the fading gain from BS $k$ (BS $k$ can either be a Macro or Micro BS) on subcarrier $z$, $\sigma^{2}$ is the noise variance, and $\left\|\mathbf{r}_{\mathbf{q}}\right\|$ is the distance from the interfering BSs; $1 \leq q \leq K$. We can define the interference term $\sum_{q=1, q \neq k}^{k} N^{2} E_{c}^{2} P_{q}\left\|\mathbf{r}_{\mathbf{q}}\right\|^{-\alpha} \sum_{z \in G_{d}}\left(\mathbf{h}_{\mathbf{q}}{ }^{(z)}\right)^{2}$ as being equal to $\mathbf{I}_{\mathbf{r}_{\mathbf{k}}}$.

In our system, part of the UEs do not undergo handover, either because they are static or because they are not within handover region. Also, part of UEs will undergo handover as they are closer to the target BSs. From geometry as shown in Fig. 7.3, assuming every cell has a radius $R$ and each UE is at a random distance $r$ from the BS, then the intersection area between the two circular cells, which represents the handover region is equal to:

$$
A_{\text {overlap }}=R^{2}(2 \theta-\sin (2 \theta))
$$

Assuming that UEs moves in a random walk motion from point $A$ at coordinates $\left(X_{A}, Y_{A}\right)$ to point $A^{\prime}$ at 
coordinates $\left(X_{A^{\prime}}, Y_{A^{\prime}}\right)$, then the angle of direction of motion $\theta$ is calculated as:

$$
\theta=\tan ^{-1} \frac{\left(Y_{A^{\prime}}-Y_{A}\right)}{\left(X_{A^{\prime}}-X_{A}\right)}
$$

The angle of direction of motion at every tier is a uniformly distributed random variable with probability density function $(\mathrm{PDF})$ equal:

$$
f_{\theta_{k}}\left(\theta_{k}\right)= \begin{cases}\frac{1}{2 \pi}, & \text { if } \pi>\theta_{k}>-\pi \\ 0, & \text { otherwise }\end{cases}
$$

Following from the fact that the null probability (probability that UE lies outside the cell) of a 2D Poisson process in an area $A_{c}$ is $\exp ^{-} \lambda_{k} A_{c}$, where $A_{c}$ is the cell area, then the probability that the UE lies inside the cell of radius $R$ is:

$$
\mathbb{P}\left(r_{k}<R\right)=1-\exp \left(-\lambda_{k} \pi R^{2}\right)
$$

Differentiating the previous equation, which is the cumulative distribution function will give the PDF of the distance between the UE and the $k$ tier BS as in [195]:

$$
f_{r_{k}}\left(r_{k}\right)= \begin{cases}2 \pi \lambda_{k} r_{k} e^{-\lambda_{k} \pi r_{k}^{2}}, & \text { if } 0<r_{k}<R . \\ 0, & \text { otherwise. }\end{cases}
$$

Assuming that area of first cell is $A_{1}$ and area of second cell is $A_{2}$, then probability of handover is:

$$
\begin{gathered}
\mathbb{P}\left(H_{k} / r_{k}, \theta_{k}\right)=1-\exp \left(-\lambda_{k}\left[A_{1} \cap A_{2}\right]\right) \\
\mathbb{P}\left(H_{k} / r_{k}, \theta_{k}\right)=1-\exp \left(-\lambda_{k} R^{2}\left(2 \theta_{k}-\sin \left(2 \theta_{k}\right)\right)\right)
\end{gathered}
$$

Similarly we can calculate the probability that no handover takes place as:

$$
\mathbb{P}\left(\bar{H}_{k} / r_{k}, \theta_{k}\right)=1-\exp \left(-\lambda_{k} R^{2}\left(2 \pi-\left(2 \theta_{k}-\sin \left(2 \theta_{k}\right)\right)\right)\right)
$$

In that situation, coverage probability for a UE if no handoff occurs is $P_{c_{\bar{H}}}$, and if handover occurs it will be $P_{c_{H}}$.

$$
\begin{gathered}
P_{c_{\bar{H}}}=\mathbb{P}\left(\gamma_{k} \geq \zeta_{k}, \bar{H}_{k} / r_{k}, \theta_{k}\right) \\
P_{c_{H}}=\mathbb{P}\left(\gamma_{k} \geq \zeta_{k}, H_{k} / r_{k}, \theta_{k}\right) \\
\mathbb{P}\left(\gamma_{k} \geq \zeta_{k}, \bar{H}_{k} / r_{k}, \theta_{k}\right)=\mathbb{E}\left[\mathbb{P}\left(\gamma_{k} \geq \zeta_{k} / r_{k}, \theta_{k}\right)\right] \cdot \mathbb{P}\left(\bar{H}_{k} / r_{k}, \theta_{k}\right)
\end{gathered}
$$




$$
\begin{gathered}
\mathbb{P}\left(\gamma_{k} \geq \zeta_{k}, H_{k} / r_{k}, \theta_{k}\right)=\mathbb{E}\left[\mathbb{P}\left(\gamma_{k} \geq \zeta_{k} / r_{k}, \theta_{k}\right)\right] \cdot \mathbb{P}\left(H_{k} / r_{k}, \theta_{k}\right) \\
\mathbb{E}\left[\mathbb{P}\left(\gamma_{k} \geq \zeta_{k} / r_{k}, \theta_{k}\right)\right]=\int_{r_{k}>0} \int_{\pi>\theta_{k}>-\pi} \mathbb{P}\left(\gamma_{k} \geq \zeta_{k} / r_{k}, \theta_{k}\right) f_{r_{k}}\left(r_{k}\right) \mathrm{d} r_{k} f_{\theta_{k}}\left(\theta_{k}\right) \mathrm{d} \theta_{k}
\end{gathered}
$$

Integration limits should go to infinity, as cell radius can take any value up to infinity [185,195]. The factor that determines this is the transmission power of the base station. As the transmission power increases, the cell radius increases. Also, direction of movement can take any angle from $-\pi$ to $\pi$.

$$
=\int_{r_{k}>0} \int_{\pi>\theta_{k}>-\pi} \mathbb{P}\left(\gamma_{k} \geq \zeta_{k} / r_{k}, \theta_{k}\right) \cdot \lambda_{k} r_{k} e^{-\lambda_{k} \pi r_{k}^{2}} \mathrm{~d} r_{k} \mathrm{~d} \theta_{k}
$$

Assume that $N^{2} E_{c}^{2} \sum_{k \in K} \lambda_{k} P_{T_{k}}=a$ then:

$$
\begin{aligned}
& =a \int_{r_{k}>0} \int_{\pi>\theta_{k}>-\pi} \mathbb{P}\left(h_{k} \geq r_{k}^{\alpha} \zeta_{k}\left(\sigma_{n}^{2}+I_{r_{k}}\right) / r_{k}, \theta_{k}\right) r_{k} e^{-\lambda_{k} \pi r_{k}^{2}} \mathrm{~d} r_{k} \mathrm{~d} \theta_{k} \\
= & a \int_{r_{k}>0} \int_{\pi>\theta_{k}>-\pi} \mathbb{E}_{\mathbf{I}_{\mathbf{r}_{\mathbf{k}}}}\left[\mathbb{P}\left(h_{k} \geq r_{k}^{\alpha} \zeta_{k}\left(\sigma_{n}^{2}+I_{r_{k}}\right) / r_{k}, \theta_{k}\right) r_{k} e^{-\lambda_{k} \pi r_{k}^{2}} \mathrm{~d} r_{k} \mathrm{~d} \theta_{k}\right]
\end{aligned}
$$

For Rayleigh fading $h_{k} \sim e^{1}$, then:

$$
\left.=a \int_{r_{k}>0} \int_{\pi>\theta_{k}>-\pi} e^{-\zeta_{k} r_{k}^{\alpha} \sigma_{n}^{2}} \mathcal{L}_{I_{r_{k}}}\left(r_{k}^{\alpha} \zeta_{k}\right) r_{k} e^{-\lambda_{k} \pi r_{k}^{2}} \mathrm{~d} r_{k} \mathrm{~d} \theta_{k}\right]
$$

where $\mathcal{L}_{I_{r_{k}}}$ is the Laplace transform of interference.

After several assumptions, we proved that Laplace transform of interference is:

$$
\mathcal{L}_{I_{r_{k}}}(S)=\frac{1}{2 \sqrt{\frac{\left(2-\frac{\pi}{2}\right) \lambda_{k} r_{k}^{1-\alpha}}{1-2 \alpha}}} e^{r_{k} \zeta_{k} \lambda_{k}\left[\frac{\left(1-\frac{\pi}{4}\right) \zeta_{k}}{1-2 \alpha}-\frac{\sqrt{\pi / 2}}{(1-\alpha)}\right]}
$$

The proof is as shown below:

Proof 7.7.1 Let $r_{k}^{\alpha} \zeta_{k}=S$, so we want to calculate Laplace transform of interference $\mathcal{L}_{I_{r_{k}}}(S)$.

$$
\mathcal{L}_{I_{r_{k}}}(S)=\int_{0}^{\infty} e^{-S \mathbf{r}_{\mathbf{k}}} \mathbf{I}\left(\mathbf{r}_{\mathbf{k}}\right) \mathrm{d} \mathbf{r}_{\mathbf{k}}=\mathbb{E}\left[e^{-S\left(\mathbf{I}_{\mathbf{r}_{\mathbf{k}}}\right)}\right]
$$

Authors in [165], proved that interference in HetNets follows a Gaussian distribution, then Laplace transform of interference will be:

$$
\mathcal{L}_{I_{r_{k}}}(S)=\frac{1}{\sqrt{2 \pi \sigma}} \int_{0}^{\infty} e^{-S \mathbf{r}_{\mathbf{k}}} e^{\frac{-\left(\mathbf{r}_{\mathbf{k}}-\mu\right)^{2}}{2 \sigma^{2}}} \mathrm{~d} \mathbf{r}_{\mathbf{k}}
$$

where $\mu$ and $\sigma^{2}$ are the mean and variance of the Gaussian distribution respectively:

$$
\mathcal{L}_{I_{r_{k}}}(S)=\frac{1}{\sqrt{2 \pi \sigma}} \int_{0}^{\infty} e^{\frac{-\frac{1}{2}\left(\mathbf{r}_{\mathbf{k}}-\mu\right)^{2}}{\sigma^{2}}-S \mathbf{r}_{\mathbf{k}}} \mathrm{d} \mathbf{r}_{\mathbf{k}}
$$


Let $v=\frac{\mathbf{r}_{\mathbf{k}}-\mu}{\sigma}$. Then, $\mathbf{r}_{\mathbf{k}}=\mu+v \sigma . \mathrm{d}\left(\mathbf{r}_{\mathbf{k}}\right)=\sigma \mathrm{d}(v)$.

$$
\mathcal{L}_{I_{r_{k}}}(S)=\frac{\sqrt{\sigma}}{\sqrt{2 \pi}} \int_{0}^{\infty} e^{-\frac{1}{2} v^{2}-S(\mu+v \sigma)} \mathrm{d} v
$$

After completing the square we get:

$$
\mathcal{L}_{I_{r_{k}}}(S)=\frac{\sqrt{\sigma}}{\sqrt{2 \pi}} e^{\frac{(S \sigma)^{2}}{2}-S \mu} \int_{0}^{\infty} e^{-\frac{1}{2}(v+S \sigma)^{2}} \mathrm{~d} v
$$

Let $u=\frac{1}{\sqrt{2}}(v+S \sigma)$, substituting by the value of $v$, and $\mathrm{d} v=\sqrt{2} \mathrm{~d} u$ we get:

$$
\mathcal{L}_{I_{r_{k}}}(S)=\frac{\sqrt{\sigma}}{\sqrt{\pi}} e^{\frac{(S \sigma)^{2}}{2}-S \mu} \int_{0}^{\infty} e^{-u^{2}} \mathrm{~d} u
$$

The integration $\int_{0}^{\infty} e^{-u^{2}} \mathrm{~d}(u)$ is evaluated as $\frac{\sqrt{\pi}}{2}$, so we substitute by its value in (7.32) we get:

$$
\mathcal{L}_{I_{r_{k}}}(S)=\frac{\sqrt{\sigma}}{2} e^{\frac{(S \sigma)^{2}}{2}-S \mu}
$$

For Rayleigh distribution, $\mu$ and $\sigma^{2}$ are equal to:

$$
\begin{gathered}
\mu=\sum_{k \in K} \lambda_{k} \mathbb{E}\left[h_{k}\right] \int_{R^{2}} r_{k}^{-\alpha} \mathrm{d}\left(r_{k}\right)=\sqrt{\frac{\pi}{2}} \sum_{k \in K} \lambda_{k} \frac{r_{k}^{(1-\alpha)}}{1-\alpha} \\
\sigma^{2}=\sum_{k \in K} \lambda_{k} \mathbb{E}\left[h_{k}^{2}\right] \int_{R^{2}}\left(r_{k}^{-\alpha}\right)^{2} \mathrm{~d}\left(r_{k}\right)=\left(2-\frac{\pi}{2}\right) \sum_{k \in K} \lambda_{k} \frac{r_{k}^{(1-2 \alpha)}}{1-2 \alpha}
\end{gathered}
$$

It follows that Laplace transform of interference is equal to:

$$
\mathcal{L}_{I_{r_{k}}}(S)=\frac{\left(\frac{\left(2-\frac{\pi}{2}\right) \lambda_{k} r_{k}^{1-\alpha}}{1-2 \alpha}\right)^{1 / 4}}{2} e^{r_{k} \zeta_{k} \lambda_{k}\left[\frac{\left(1-\frac{\pi}{4}\right) \zeta_{k}}{1-2 \alpha}-\frac{\sqrt{\pi / 2}}{(1-\alpha)}\right]}
$$

Then, we substitute by (7.26) into (7.25) so:

$$
\mathbb{E}\left[\mathbb{P}\left(\gamma_{k} \geq \zeta_{k} / r_{k}, \theta_{k}\right)\right]=2 \pi a \int_{r_{k}>0} e^{-\zeta_{k} r_{k}^{\alpha} \sigma_{n}^{2}} \mathcal{L}_{I_{r_{k}}}\left(r_{k}^{\alpha} \zeta_{k}\right) r_{k} e^{-\lambda_{k} \pi r_{k}^{2}} \mathrm{~d} r_{k}
$$

Then, we substitute by (7.37), (7.15), and (7.16) into (7.19), and (7.20) to get $\mathbb{P}\left(\gamma_{k} \geq \zeta_{k}, \bar{H}_{k} / r_{k}, \theta_{k}\right)$ and $\mathbb{P}\left(\gamma_{k} \geq \zeta_{k}, H_{k} / r_{k}, \theta_{k}\right)$.

$$
\begin{gathered}
\mathbb{P}\left(\gamma_{k} \geq \zeta_{k}, \bar{H}_{k} / r_{k}, \theta_{k}\right)=2 \pi a\left(1-\exp \left(-\lambda_{k} R^{2}\left(2 \pi-\left(2 \theta_{k}-\sin \left(2 \theta_{k}\right)\right)\right)\right)\right) \int_{r_{k}>0} e^{-\zeta_{k} r_{k}^{\alpha} \sigma_{n}^{2}} \mathcal{L}_{I_{r_{k}}}\left(r_{k}^{\alpha} \zeta_{k}\right) r_{k} e^{-\lambda_{k} \pi r_{k}^{2}} \mathrm{~d} r_{k} \\
\mathbb{P}\left(\gamma_{k} \geq \zeta_{k}, H_{k} / r_{k}, \theta_{k}\right)=2 \pi a\left(1-\exp \left(-\lambda_{k} R^{2}\left(2 \theta_{k}-\sin \left(2 \theta_{k}\right)\right)\right)\right) \int_{r_{k}>0} e^{-\zeta_{k} r_{k}^{\alpha} \sigma_{n}^{2}} \mathcal{L}_{I_{r_{k}}}\left(r_{k}^{\alpha} \zeta_{k}\right) r_{k} e^{-\lambda_{k} \pi r_{k}^{2}} \mathrm{~d} r_{k}
\end{gathered}
$$




\subsection{Simulation Analysis}

Matlab $^{T M}$ simulation was used to analyze the performance of RTLB UE association techniques. We evaluate the efficacy of the proposed methods in comparison with the conventional max-SINR algorithm, SINR biased, and rate biased algorithms. Macro BSs, Micro BSs, and UEs are scattered uniformly in the working area according to three homogeneous dependant PPP using parent-child relation-ship. Table 7.1 lists the values we used in estimating the performance of the proposed method.

Next, we analyze the performance of the suggested real time UE association algorithm. We initially deploy 500 UEs uniformly according to the model shown in Fig. 7.4. Fig. 7.7, compares the performance of the elapsed time that both algorithms will take. We show that in real-time scenarios and when the system configuration is changing rapidly, RTLB algorithm performs very fast. LSTD performs very well for a certain configuration in a reasonable time, but the problem is that when the data set configuration changes at a certain moment (some users are dropped and some are added) we have to run the algorithm all over again from the beginning. Repeating LSTD algorithm is not a problem for small changes and for short time, but accumulatively through a long time and many iterations there will be a time delay that can affect system performance. RTLB can be a good option in real-time scenarios where the network configuration changes rapidly and continuously.

Fig. 7.8 presents the simulation for BER for OFCDM link for 32 UEs and 16 Micro BSs. BSs are using the same number of subcarriers and the spreading factor in time domain is 4 . First UEs send data streams to Micro BSs. User association is decided by the Macro BS in a centralized manner where Macro BS receives the original SINR from Micro BSs and generates the SINR matrix. UEs are associated to Micro BSs based

Table 7.1: Parameters used for RTLB user association algorithm

\begin{tabular}{|c|c|}
\hline Parameter & Value \\
\hline$\lambda_{m}$ & 20 \\
\hline$\lambda_{u}$ & 40 \\
\hline Micro Cell Radius & $500(\mathrm{~m})$ \\
\hline Coverage Area & $4000 \times 4000\left(\mathrm{~m}^{2}\right)$ \\
\hline Min Distance Between Micro BSs & $400(\mathrm{~m})$ \\
\hline Total Number of Users & 500 \\
\hline Initial Number of Active Users & 50 \\
\hline Macro BS Transmit Power & $50($ Watt $)$ \\
\hline Noise Power & $20($ Watt $)$ \\
\hline
\end{tabular}




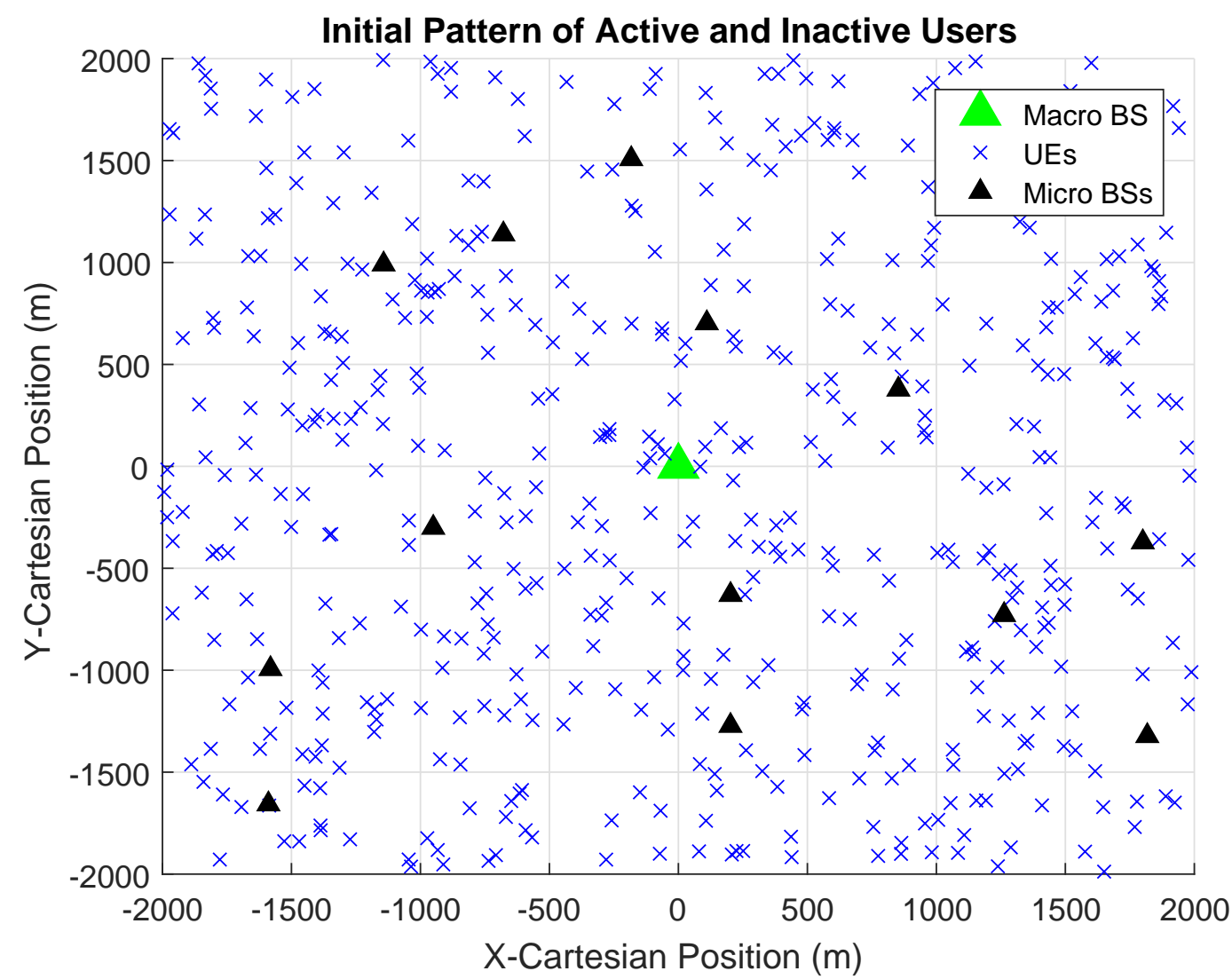

Figure 7.4: Initial data set for RTLB model for a scaled grid area of $4000 \times 4000 \mathrm{~m}$ and 500 total UEs

on max-SINR and then using our suggested algorithm. BER is significantly reduced with our algorithm.

Fig. 7.10 and Fig. 7.11 present the load distribution along BSs for our proposed RTLB algorithm compared with various algorithms. Both RTLB and LSTD algorithms provide fairly balanced load distribution. However, in real-time scenarios RTLB outperforms LSTD as it is faster, easily adapts to rapid network changes, and provides adequate balanced UE distribution among BSs. Max-SINR user association tends to allocate more UEs to the Macro BS and less load to the Micro BSs. Load imbalance among tier BSs is clearly seen with this algorithm. SINR-biased user association algorithm improves the performance of max-SINR, but does not provide optimal fair distribution. The value of the optimum bias value has to be investigated. Finally, rate-biased algorithm works the opposite way of max-SINR as it tends to associate UEs to small cell BSs. The optimum bias value has to be calculated considering required performance metrics.

From Fig. 7.12, for max-SINR most UEs select the Macro BS so less low-rate UEs are available. CRE and rate-biased provide a significant improvement over max-SINR, where more UEs are offloaded from Macro to Micro BSs due to the biasing factor. As a result, more resources are available for Macro BS UEs and more 


\begin{tabular}{|c|c|c|c|c|c|c|c|c|c|c|c|c|c|c|c|c|c|c|c|c|c|c|c|c|c|c|c|c|c|c|}
\hline Iterations & Load Threshold & \multicolumn{14}{|c|}{ BS Status } & \multicolumn{14}{|c|}{ udist } & Std(udist) \\
\hline 1 & 6 & 1 & 1 & 0 & 1 & 0 & 1 & 1 . & & 1 & 1 & 1 & 1 & 1 & 1 & 2 & 2 & 6 & 4 & 6 & 3 & 4 & 7 & 3 & 3 & 3 & 2 & 4 & 4 & 1.685425567 \\
\hline 2 & 7 & 1 & 1 & 0 & 1 & 0 & 1 & 1 & 0 & 1 & 1 & 1 & 1 & 1 & 1 & 2 & 4 & 7 & 5 & 7 & 4 & 3 & 7 & 3 & 3 & 3 & 2 & 4 & 4 & 2.259290952 \\
\hline 3 & 6 & 1 & 1 & 0 & 1 & 0 & 1 & 1 & 1 & 1 & 1 & 1 & 1 & 1 & 1 & 2 & 4 & 6 & 4 & 6 & 4 & 3 & 5 & 3 & 2 & 3 & 2 & 3 & 3 & 2.248320496 \\
\hline 4 & 6 & 1 & 1 & 1 & 1 & 0 & 1 & $1-$ & 1 & 1 & 1 & 1 & 1 & 1 & 1 & 2 & 4 & 5 & 4 & 6 & 4 & 3 & 7 & 3 & 2 & 2 & 3 & 3 & 3 & 2.334641182 \\
\hline 5 & 6 & 1 & 0 & 0 & 0 & 1 & 1 & 1 & 0 & 1 & 1 & 1 & 1 & 1 & 1 & 2 & 6 & 6 & 6 & 5 & 4 & 4 & 6 & 3 & 1 & 3 & 4 & 2 & 3 & 2.340517459 \\
\hline 6 & 7 & 1 & 0 & 1 & 0 & 1 & 1 & 1. & 1 & 1 & 1 & 1 & 1 & 1 & 1 & 2 & 7 & 6 & 7 & 6 & 3 & 4 & 8 & 4 & 1 & 4 & 3 & 1 & 3 & 2.173769704 \\
\hline 7 & 7 & 1 & 0 & 0 & 0 & 1 & 1 & 1 & 0 & 1 & 1 & 1 & 1 & 1 & 1 & 4 & 7 & 7 & 7 & 6 & 3 & 3 & 7 & 3 & 1 & 4 & 2 & 1 & 3 & 1.857565463 \\
\hline 8 & 7 & 1 & 0 & 0 & -1 & 0 & 1 & 1 & 1 & 1 & 1 & 1 & 1 & 1 & 1 & 4 & 7 & 7 & 8 & 7 & 4 & 3 & 6 & 3 & 1 & 4 & 2 & 1 & 3 & 1.899971081 \\
\hline 9 & 7 & 1 & 1 & 0 & -1 & 0 & 1 & 1. & 1 & 1 & 1 & 1 & 1 & 1 & 1 & 4 & 5 & 7 & 8 & 7 & 4 & 5 & 9 & 3 & 1 & 4 & 2 & 3 & 3 & 1.589802669 \\
\hline 10 & 7 & 1 & 1 & -1 & 1 & 0 & 1 & 1. & 1 & 1 & 1 & 1 & 1 & 1 & 1 & 4 & 4 & 8 & 6 & 7 & 5 & 3 & 9 & 3 & 2 & 4 & 4 & 2 & 3 & 1.730463954 \\
\hline 11 & 7 & 1 & 1 & 1 & 1 & 1 & 1 & $1-$ & 1 & 1 & 1 & 1 & 1 & 1 & 1 & 4 & 5 & 6 & 6 & 5 & 4 & 3 & 9 & 4 & 2 & 4 & 3 & 2 & 3 & 1.899971081 \\
\hline 12 & 7 & 1 & 1 & 0 & 1 & 1 & 1 & $1-$ & 1 & 1 & 1 & 1 & 1 & 1 & 1 & 2 & 5 & 7 & 5 & 6 & 3 & 3 & 8 & 4 & 2 & 4 & 3 & 2 & 3 & 1.977899874 \\
\hline 13 & 6 & 1 & 1 & 0 & 1 & 1 & 1 & $1-$ & 1 & 1 & 1 & 1 & 1 & 1 & 1 & 2 & 4 & 6 & 5 & 5 & 3 & 3 & 7 & 4 & 2 & 4 & 2 & 2 & 3 & 1.743118337 \\
\hline 14 & 6 & 1 & 1 & 0 & 0 & 1 & 1 & $1-$ & 1 & 1 & 1 & 1 & 1 & 1 & 1 & 3 & 4 & 6 & 6 & 5 & 4 & 3 & 7 & 4 & 1 & 5 & 2 & 2 & 3 & 2.054210364 \\
\hline 15 & 7 & 1 & 1 & 0 & 0 & 1 & 1 & 1 & 1 & 1 & 1 & 1 & 1 & 1 & 1 & 4 & 4 & 7 & 7 & 5 & 4 & 3 & 6 & 5 & 1 & 5 & 2 & 2 & 2 & 2.070196678 \\
\hline
\end{tabular}

Figure 7.5: An illustration of algorithm performance along iterations as user calls are added or dropped

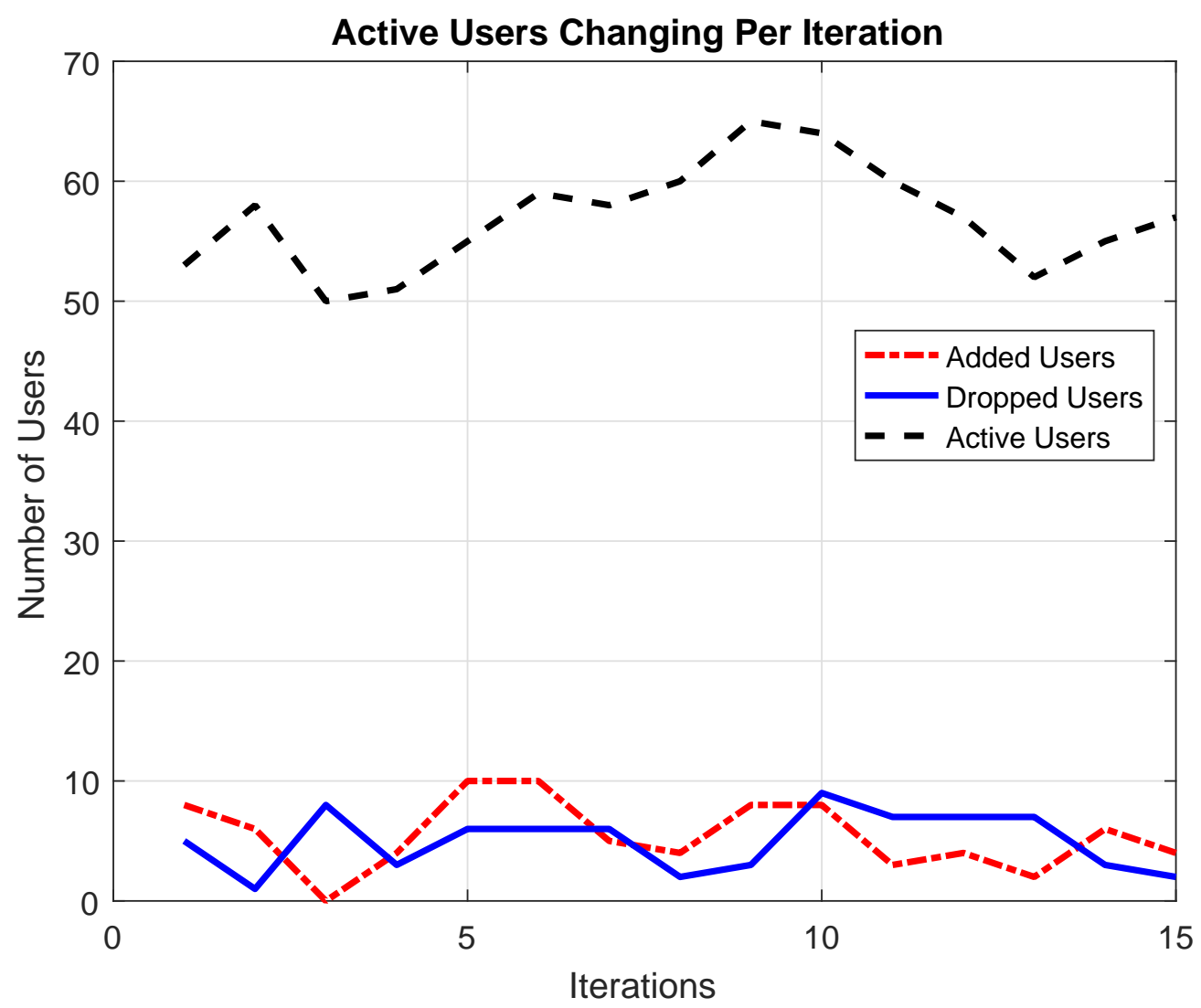

Figure 7.6: An illustration of how our proposed algorithm supports variation in active users 


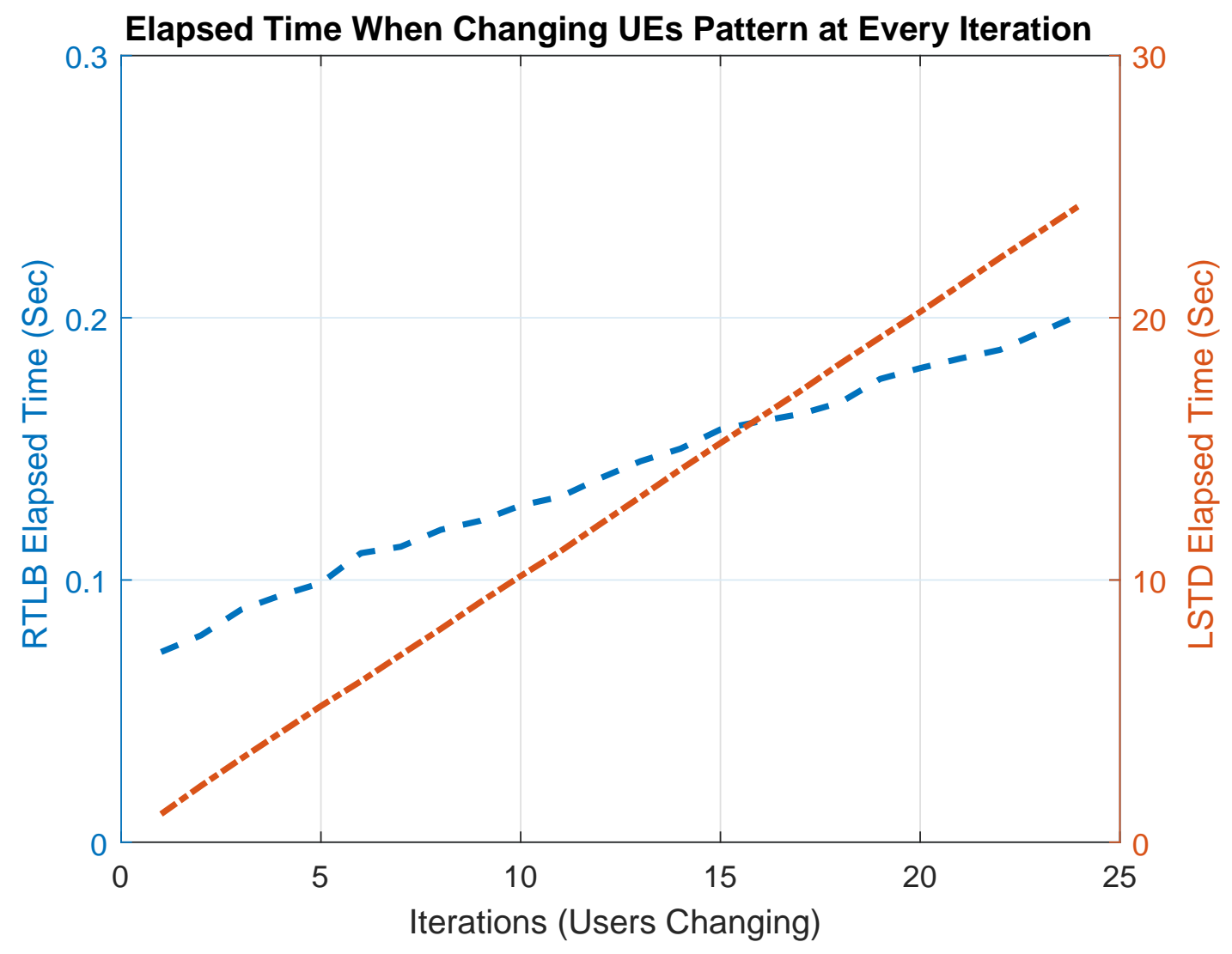

Figure 7.7: An illustration of the elapsed time for RTLB versus LSTD

low-rate UEs are available. In our proposed algorithm, an optimum solution is achieved where nearly equal resources are available for Macro and Micro UEs and there are nearly equal low-rate and high-rate UEs.

\subsection{Chapter Summary}

In this chapter, we developed a dynamic fast UE association algorithm for cooperating BSs in HetNets considering UEs mobility, and UEs and BSs status changes. As UEs mobility is considered, we derive an expression for coverage probability if UEs undergo handover when they are in handover range and when they do not undergo handover when they are outside this range. Our results are feasible in the real wireless network systems that would help in the development of future $5 \mathrm{G}$ systems. 


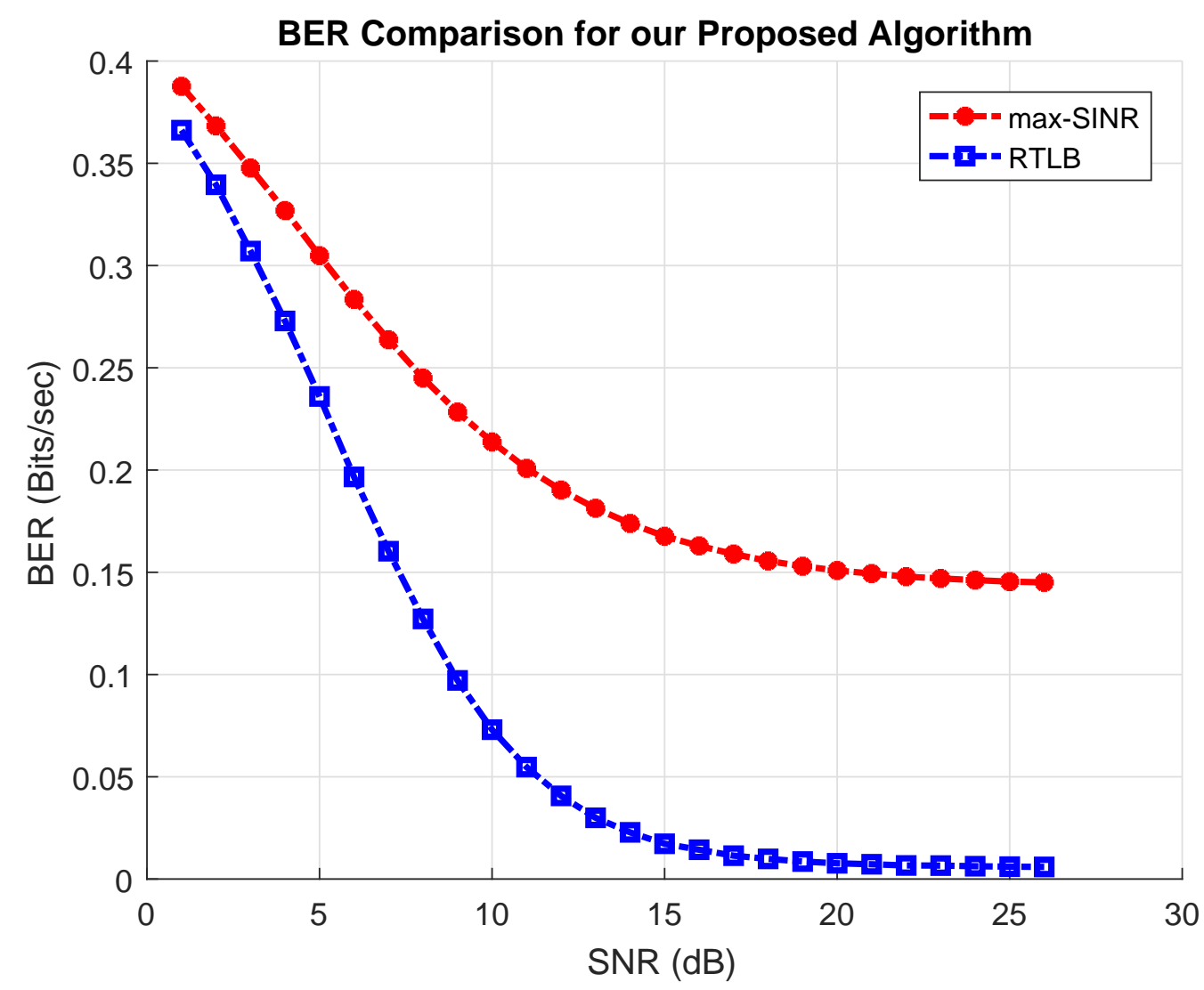

Figure 7.8: BER Comparison for RTLB versus max-SINR 


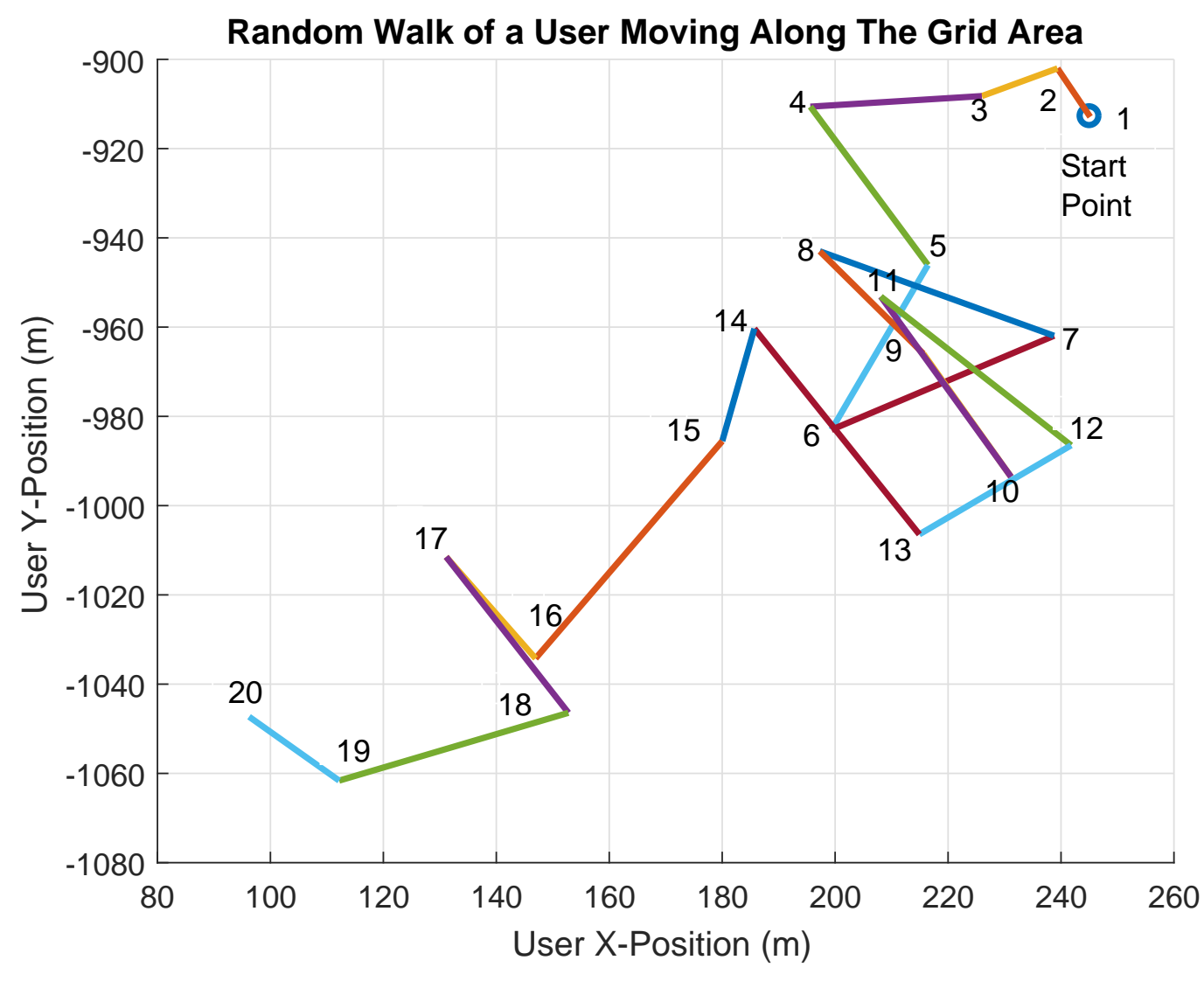

Figure 7.9: Proposed Algorithm supports users' motion 


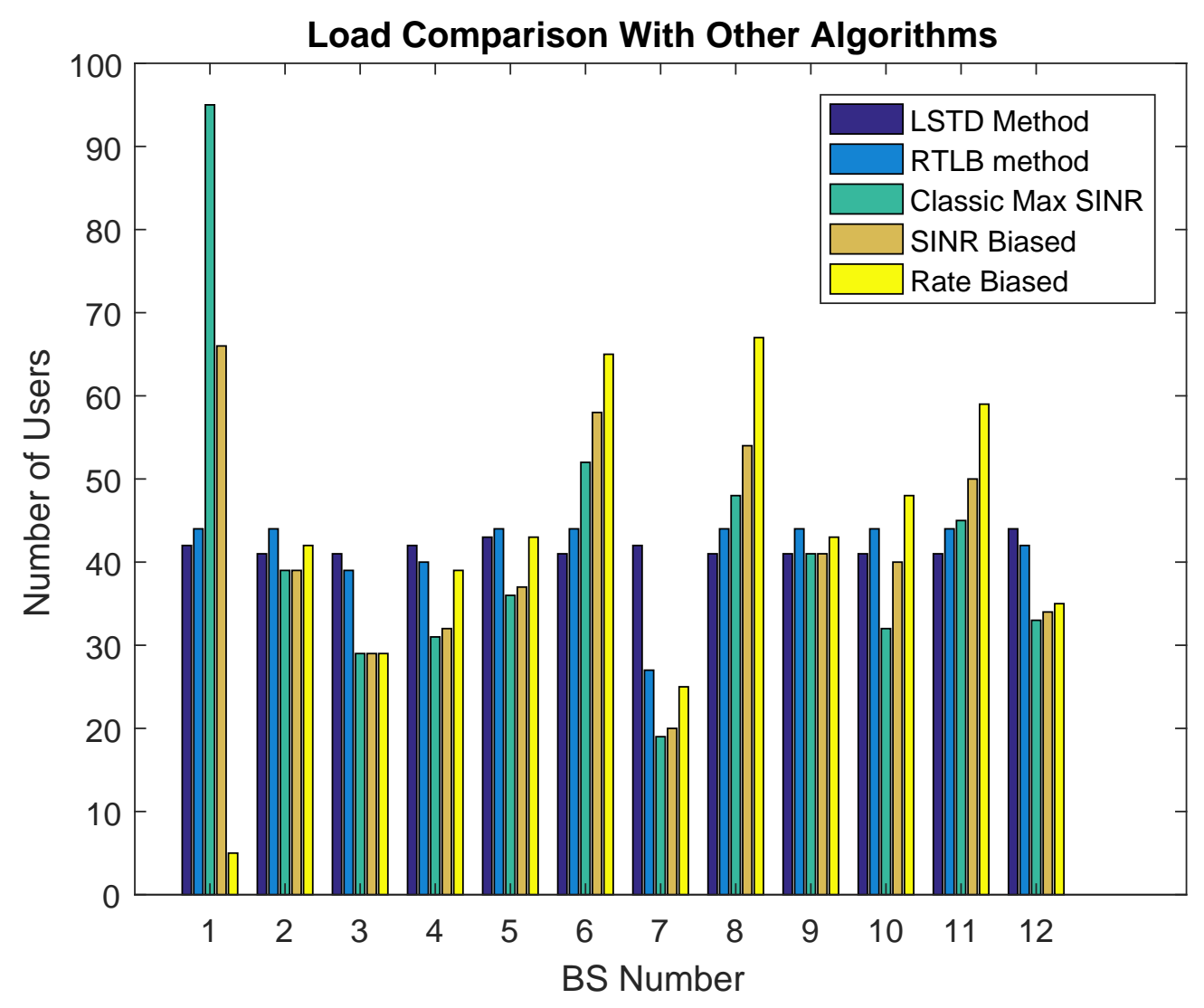

Figure 7.10: Comparison between network load of the proposed algorithm versus other algorithms for $\operatorname{bias}=1.5$ 


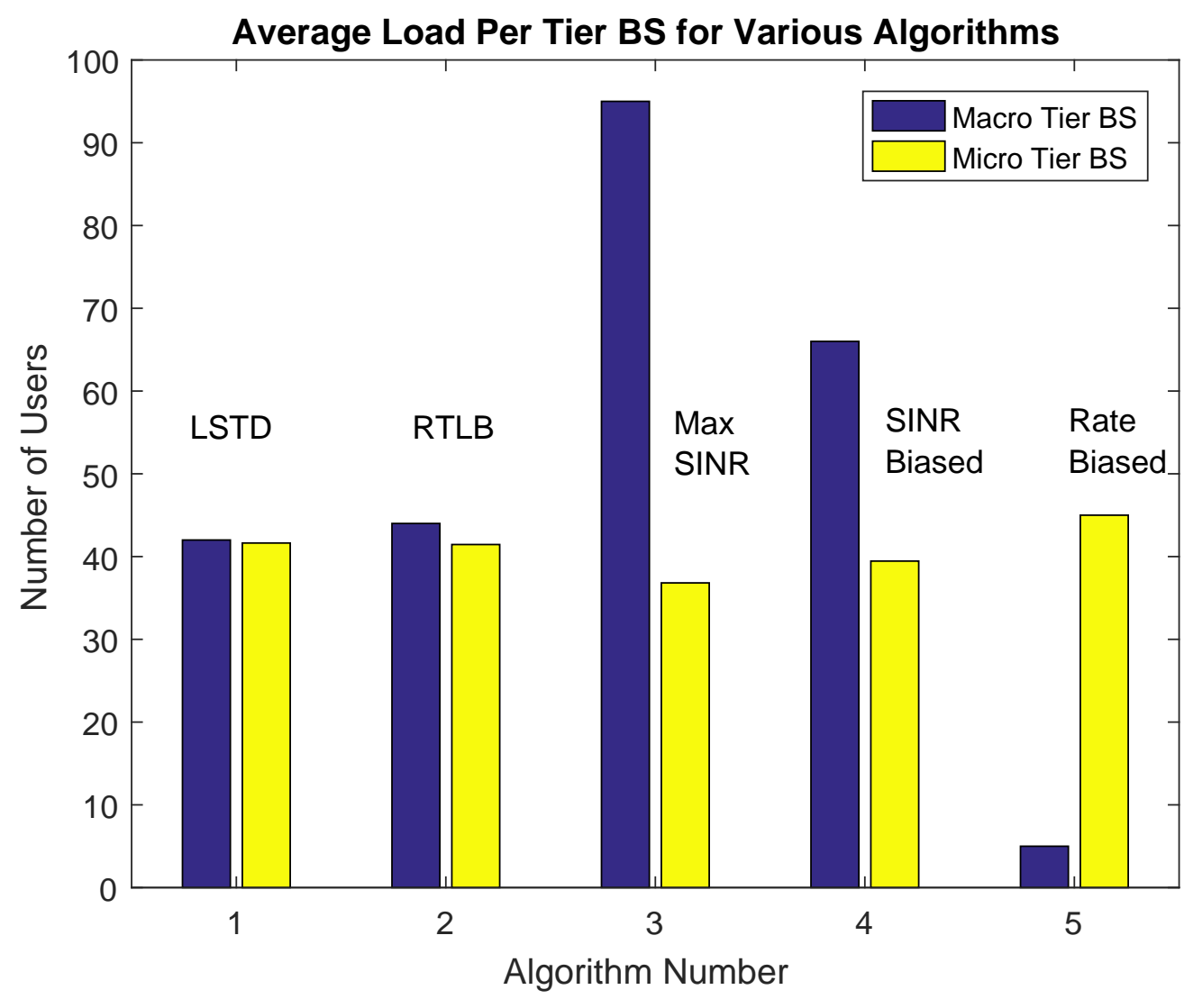

Figure 7.11: Average Load Per Tier BS Comparison 


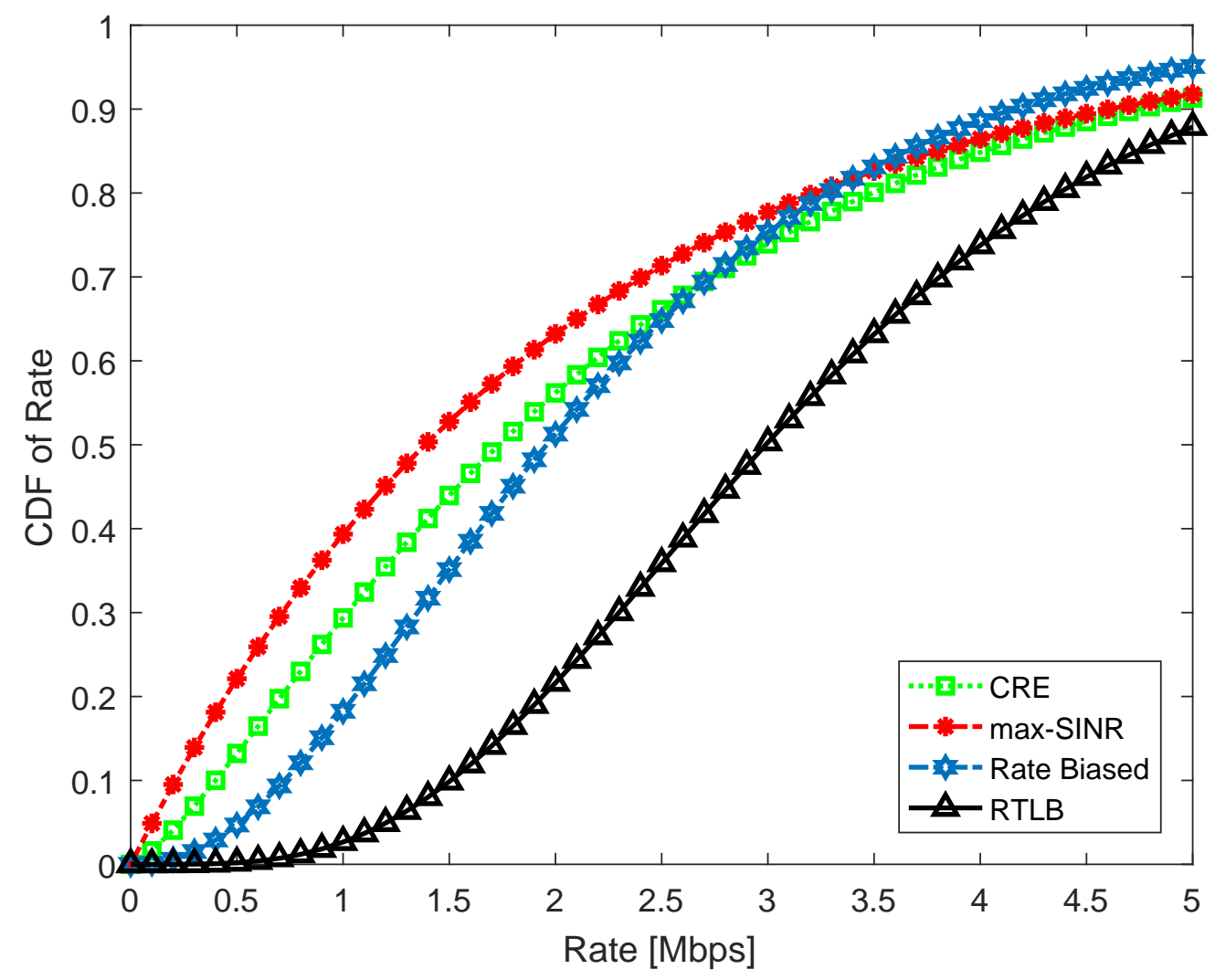

Figure 7.12: CDF of rate comparison for various association algorithms 


\section{CHAPTER 8}

\section{Discussion and Future Work}

\subsection{Objectives and Contributions}

The main objective of this dissertation was to enhance the performance, combat high interference levels in ultra dense HetNet systems, and to design user association algorithms to provide fair load distribution despite the inhomogeneity of the system. Our goal was achieved using the following contributions:

- Optimizing pilot sequence length to improve data rate and reduce MSE. Optimum pilot sequence length depends on number of users.

- Suggesting an algorithm to optimize Micro BSs locations to minimize interference from adjacent BSs and increase coverage.

- Suggesting an algorithm to optimize Macro and core Micro BSs to eliminate interference. We were able to completely isolate every Macro cell along with all its associated Micro BSs and treat it as an independent unit.

- Suggesting a UE association algorithm to fair load distribution by minimizing network load standard deviation. User association resulted in nearly constant load distribution in all the network. Our algorithm has acceptable performance for real time scenarios and rapid network changes. The execution time per data set change was nearly 1 sec.

- Suggesting a real time dynamic UE association algorithm assuming that traffic transfer is possible from heavy loaded to lightly loaded BSs and vice versa. Also, we assume that BSs are cooperating and broadcasting their load information continuously. In our algorithm, we considered UEs mobility, UEs status change, and BSs status change. We compared the performance of our algorithms to other work 
in literature and proved the superiority of our algorithms in providing balanced load among all BSs in a timely manner. The execution time per every data set change was about $0.02-0.07 \mathrm{sec}$.

- We derived coverage probability for static users, network energy efficiency, drop rate, coverage probability for mobile users considering handover and no handover cases.

All aforementioned contributions were preceded by a deep analysis of the existing work in literature. This allowed the identification of the advantages, weaknesses, trends and challenges related to the HetNet development.

\subsection{Future Developments}

HetNet technology is an emerging solution with huge potential for 5G systems. HetNets would provide connectivity between various network layers with each layer considered as an individual system with its own access methods, transmission powers, and topology. HetNets have their own unique characteristics that differ them from conventional homogeneous systems. BSs are closely packed which increases interference levels dramatically and due to the variation in signal power of various tier BSs, conventional UE association algorithms does not work properly with it. For the future work, we can expand our system according to the following:

- Benefit form the characteristics of OFCDM (UEs can transmit different data types with a different code for each one) and consider multi-classes of UEs, where UEs can ve divided into UEs sending data, video, and audio at the same time and study whether the proposed UE association algorithms can provide adequate performance with the new assumptions.

- As we generate SINR matrix for the possibilities of UEs connection and assign 1 for greater than a threshold and 0 for less than a threshold, we can apply error correcting output coding which can be used for multiple class classification to classify UEs and assign them to BSs based on some features based on the assumption that UEs carry different data types.

- Apply power allocation and subcarrier allocation for the OFCDM HetNet using Q-learning machine learning algorithm.

- Optimize number of BSs in every tier and study its effect on battery life and energy efficiency.

- Introduce more traffic management, radio resource management, and network planning in HetNets.

- Study new optimum beam forming, precoding, estimation, and blind detection methods for HetNets. New algorithms to mitigate pilot contamination.

- Study various performance metrics in HetNets in the massive MIMO context. 


\subsection{Conclusions}

In this dissertation, we introduced a novel analytical method to obtain the optimum pilot length under pilot power and NMSE constraints. Optimum pilot length depends on number of users. Longer pilot length gives more channel capacity and NMSE. A moderate value of pilot sequence length is recommended to achieve both good data rate and NMSE. However, overly long pilot will impair the system. Hence, a suitable training length should be chosen according to the system requirements.

Also, we derived analytical expressions for coverage probability and energy efficiency for HetNets and we introduced an algorithm to optimize Micro BSs locations to improve coverage, energy efficiency, and reduce interference. We put constraints on location of Micro BSs within the cell area used interior-reflective Newton optimization method to solve our non-linear optimization problem. Our results showed significant improvement in system performance by eliminating interference.

Furthermore, we suggested an algorithm to optimize Macro BSs locations by eliminating all sources of interference coming from adjacent Macro BSs, Micro BSs in adjacent cells, and edge Micro BSs in the same cell. We succeeded to obtain Macro cells as interference free and completely independent units from the rest of the network. After achieving the previous goal, we worked on core Micro BSs in the same cell and obtained their best positions to reduce interference as well. We supported our findings by Matlab images and figures to show how we were able to achieve a completely interference free HetNet system.

Finally, we introduced two UE association algorithms for load balancing in HetNets. The first algorithm works by minimizing overall network standard deviation. First we specify all UEs possibilities of connection based on a certain SINR threshold, then we sort UEs in ascending order by the ones with the least possibilities. We try to assign first UE to the least loaded BS then the more loaded one and so on and calculate standard deviation in every iteration until a balanced load is achieved.

The second algorithm works by balancing load as well by borrowing users and giving users to other BSs and by the idea that traffic transfer is possible between BSs. We first start by specifying which BSs are in giving state and specify which UEs are associated with them. UEs with the highest SINR value should be given first. Specify BSs which are in accepting state and at the same time it is one of the available BSs of that UE for connection. Move the UE to the one with the highest SINR value.

When comparing both algorithms we concluded that they both provide balanced load distribution. LSTD runs in nearly $1 \mathrm{sec}$ per data set change, and RTLB runs in nearly $0.02 \mathrm{sec}$ per data set change. Both algorithms run fast enough so that no significant delay is detected by the serviced UEs. Both algorithms consider an acceptable SINR level for the BS that UE should be moved to, as well as, network load. In real time rapid changing systems, RTLB might be more favourable. 


\section{Bibliography}

[1] André Martins, António Rodrigues, and Pedro Vieira. On the choice of positioning and cluster size for fixed relay stations in a LTE network. Wireless personal communications, 79(1):745-762, 2014.

[2] Emil Björnson, Erik G Larsson, and Thomas L Marzetta. Massive MIMO: Ten myths and one critical question, 2015.

[3] Erik Larsson, Ove Edfors, Fredrik Tufvesson, and Thomas Marzetta. Massive MIMO for next generation wireless systems. Communications Magazine, IEEE, 52(2):186-195, 2014.

[4] AKHIL Gupta and RAKESH KUMAR Jha. A survey of 5G network: architecture and emerging technologies. Access, IEEE, 3:1206-1232, 2015.

[5] Lu Lu, Geoffrey Ye Li, A Lee Swindlehurst, Alexei Ashikhmin, and Rui Zhang. An overview of massive MIMO: Benefits and challenges. Selected Topics in Signal Processing, IEEE Journal of, 8(5):742-758, 2014 .

[6] A Chockalingam and B Sundar Rajan. Large MIMO Systems. Cambridge University Press, 2014.

[7] Jeffrey G Andrews, Stefano Buzzi, Wan Choi, Stephen V Hanly, Aurelie Lozano, Anthony CK Soong, and Jianzhong Charlie Zhang. What will 5G be? Selected Areas in Communications, IEEE Journal on, 32(6):1065-1082, 2014.

[8] Dantong Liu, Lifeng Wang, Yue Chen, Maged Elkashlan, Kai-Kit Wong, Robert Schober, and Lajos Hanzo. User association in $5 \mathrm{G}$ networks: A survey and an outlook. IEEE Communications Surveys $\mathbb{E}$ Tutorials, 18(2):1018-1044, 2016.

[9] Thomas L Marzetta. Massive MIMO: An introduction. Bell Labs Technical Journal, 20:11-22, 2015.

[10] Sau-Hsuan Wu. Factor graph em algorithm for joint channel tracking and map detection of MIMO-OFDMA in fading channels. In Acoustics, Speech and Signal Processing, 2008. ICASSP 2008. IEEE International Conference on, pages 2701-2704. IEEE, 2008. 
[11] Xiang Xu et al. Factor graph based detection and channel estimation for MIMO-OFDM Systems in doubly selective channel. In 2010 7th International Symposium on Wireless Communication Systems, pages 456-460, 2010.

[12] Bernhard Etzlinger, Werner Haselmayr, and Andreas Springer. Message passing methods for factor graph based MIMO detection. In Wireless Advanced (WiAd), 2011, pages 132-137. IEEE, 2011.

[13] T Lakshmi Narasimhan, Ananthanarayanan Chockalingam, and B Sundar Rajan. Factor graph based joint detection/decoding for ldpc coded large-MIMO Systems. In Vehicular Technology Conference (VTC Spring), 2012 IEEE 75th, pages 1-5. IEEE, 2012.

[14] Werner Haselmayr, Bernhard Etzlinger, and Andreas Springer. Factor-graph-based soft-input soft-output detection for frequency-selective MIMO channels. Communications Letters, IEEE, 16(10):1624-1627, 2012.

[15] Clemens Novak, Gerald Matz, and Franz Hlawatsch. Idma for the multiuser MIMO-OFDM uplink: a factor graph framework for joint data detection and channel estimation. Signal Processing, IEEE Transactions on, 61(16):4051-4066, 2013.

[16] Xiangming Meng, Sheng Wu, Linling Kuang, Zuyao Ni, and Jianhua Lu. Expectation propagation based iterative multi-user detection for mimo-idma systems. In Vehicular Technology Conference (VTC Spring), 2014 IEEE 79th, pages 1-5. IEEE, 2014.

[17] Sana Sfar and Khaled Letaief. Group ordered successive interference cancellation for multiuser detection in MIMO CDMA Systems. In Wireless Communications and Networking, 2003. WCNC 2003. 2003 IEEE, volume 2, pages 888-893. IEEE, 2003.

[18] Wen Che, Hui Zhao, and Wenbo Wang. An improved soft interference cancellation based combined probability data association and sphere decoding algorithm for MIMO detection. In Communications and Networking in China, 2007. CHINACOM'07. Second International Conference on, pages 1106-1110. IEEE, 2007.

[19] Li Wang, Lei Xu, Sheng Chen, and Lajos Hanzo. MMSE soft-interference-cancellation aided iterative center-shifting k-best sphere detection for MIMO channels. In Communications, 2008. ICC'08. IEEE International Conference on, pages 3819-3823. IEEE, 2008.

[20] Manabu Mikami and Teruya Fujii. Iterative MIMO signal detection with inter-cell interference cancellation for downlink transmission in coded OFDM cellular systems. In Vehicular Technology Conference, 2009. VTC Spring 2009. IEEE 69th, pages 1-5. IEEE, 2009. 
[21] Ke Xiao, Mingchao Su, and Shujun Guo. MMSE soft-interference-cancellation aided MCMC detection for MIMO Systems. In Industrial Engineering and Engineering Management, 2009. IEGEM'09. 16th International Conference on, pages 2107-2110. IEEE, 2009.

[22] Christoph Studer, Schekeb Fateh, and Dominik Seethaler. Asic implementation of soft-input soft-output MIMO detection using MMSE parallel interference cancellation. Solid-State Circuits, IEEE Journal of, 46(7):1754-1765, 2011.

[23] Peng Li, Rodrigo C De Lamare, and Rui Fa. Multiple feedback successive interference cancellation detection for multiuser MIMO Systems. Wireless Communications, IEEE Transactions on, 10(8):2434-2439, 2011.

[24] Yung-Ping Tu, Hoang-Yang Lu, and Wen-Hsien Fang. Alternating multiuser detection with soft interference cancellation for heterogeneous-signaling MIMO CDMA Systems. Wireless Personal Communications, 67(4):811-828, 2012.

[25] Yejian Chen and Stephan Ten Brink. Enhanced MIMO subspace detection with interference cancellation. In Wireless Communications and Networking Conference (WCNC), 2012 IEEE, pages 267-271. IEEE, 2012.

[26] Bei Yin and Joseph R Cavallaro. LTE uplink MIMO receiver with low complexity interference cancellation. Analog Integrated Circuits and Signal Processing, 73(2):443-450, 2012.

[27] Keke Zu and Rodrigo C De Lamare. Pre-sorted multiple-branch successive interference cancelation detection for high-dimensional MIMO Systems. In Smart Antennas (WSA), 2012 International ITG Workshop on, pages 157-161. IEEE, 2012.

[28] Jie Wu, Jie Zhong, Yunlong Cai, Minjian Zhao, and Wenyan Zhang. New detection algorithms based on the jointly gaussian approach and successive interference cancelation for iterative MIMO Systems. International Journal of Communication Systems, 27(10):1964-1983, 2014.

[29] Jin Xu, Xiaoming Dai, Weiguo Ma, and Yingmin Wang. A component-level soft interference cancellation based iterative detection algorithm for coded MIMO Systems. In Vehicular Technology Conference (VTC Fall), 2014 IEEE 80th, pages 1-5. IEEE, 2014.

[30] Liliana Arevalo, Rodrigo C de Lamare, Keke Zu, and Raimundo Sampaio-Neto. Multi-branch lattice reduction successive interference cancellation detection for multiuser MIMO Systems. In Wireless Communications Systems (ISWCS), 2014 11th International Symposium on, pages 219-223. IEEE, 2014 . 
[31] Manish Mandloi, Mohammed Azahar Hussain, and Vimal Bhatia. Adaptive multiple stage k-best successive interference cancellation algorithm for mimo detection. Telecommunication Systems, pages $1-16,2017$.

[32] Frederik Simoens, Henk Wymeersch, and Marc Moeneclaey. A novel mimo detection scheme with linear complexity. In Wireless Communications and Networking Conference, 200\%. WCNC 200\%. IEEE, pages 1103-1107. IEEE, 2007.

[33] Ni-Chun Wang, Ezio Biglieri, and Kung Yao. A systolic array for linear MIMO detection based on an all-swap lattice reduction algorithm. In Acoustics, Speech and Signal Processing, 2009. ICASSP 2009. IEEE International Conference on, pages 2461-2464. IEEE, 2009.

[34] Chiao-En Chen and Wern-Ho Sheen. A new lattice reduction algorithm for lr-aided MIMO linear detection. Wireless Communications, IEEE Transactions on, 10(8):2417-2422, 2011.

[35] Chengkang Pan, Jian Geng, Guangyi Liu, Jianjun Liu, Qixing Wang, and Xiaodong Shen. Linear detection and precoding for physical network coding in two-way MIMO relay channels. In Vehicular Technology Conference (VTC Fall), 2011 IEEE, pages 1-4. IEEE, 2011.

[36] Hao-Hsiang Chung, Shiuan-Hao Kuo, and Mao-Chao Lin. A physical-layer network coding scheme based on linear MIMO detection. In Vehicular Technology Conference (VTC Spring), 2012 IEEE 75th, pages 1-5. IEEE, 2012.

[37] Bei Yin, Min Wu, Christoph Studer, Joseph R Cavallaro, and Chris Dick. Implementation trade-offs for linear detection in large-scale MIMO Systems. In Acoustics, Speech and Signal Processing (ICASSP), 2013 IEEE International Conference on, pages 2679-2683. IEEE, 2013.

[38] Keke $\mathrm{Zu}$ and Rodrigo $\mathrm{C}$ de Lamare. Adaptive switched lattice reduction-aided linear detection techniques for MIMO Systems. arXiv preprint arXiv:1304.6468, 2013.

[39] Yunlong Cai and Rodrigo C de Lamare. Adaptive minimum BER reduced-rank linear detection for massive MIMO Systems. arXiv preprint arXiv:1302.4433, 2013.

[40] Youngmin Kim, Jae Hyun Seo, Heung Mook Kim, and Sooyoung Kim. Soft linear MMSE detection for coded MIMO Systems. In Communications (APCC), 2013 19th Asia-Pacific Conference on, pages 657-660. IEEE, 2013.

[41] Shahram Zarei, Wolfgang H Gerstacker, Jocelyn Aulin, and Robert Schober. I/q imbalance aware widely-linear receiver for uplink multi-cell massive mimo systems: Design and sum rate analysis. IEEE Transactions on Wireless Communications, 15(5):3393-3408, 2016. 
[42] Leonardo D Oliveira, Paul Jean E Jeszensky, Taufik Abrao, Fernando Casadevall, et al. Simplified local search multiuser detection for QPSK S/MIMO MC-CDMA Systems. In Signal Processing and Communication Systems, 2008. ICSPCS 2008. 2nd International Conference on, pages 1-6. IEEE, 2008.

[43] I-Wei Lai, Chia-Han Lee, Gerd Ascheid, Heinrich Meyr, and Tzi-Dar Chiueh. Channel-aware local search (CA-LS) for iterative MIMO detection. In Personal, Indoor, and Mobile Radio Communications (PIMRC), 2015 IEEE 26th Annual International Symposium on, pages 731-736. IEEE, 2015.

[44] Ali Elghariani and Michael Zoltowski. Low complexity detection algorithms in large-scale mimo systems. IEEE Transactions on Wireless Communications, 15(3):1689-1702, 2016.

[45] Lin Li, Weixiao Meng, and Cheng Li. Semidefinite further relaxation on likelihood ascent search detection algorithm for high-order modulation in massive mimo system. IET Communications, 11(6):801-808, 2016.

[46] Vishakan Ponnampalam, Darren McNamara, Andy Lillie, and Magnus Sandell. On generating soft outputs for lattice-reduction-aided MIMO detection. In Communications, 200\%. ICC'0\%. IEEE International Conference on, pages 4144-4149. IEEE, 2007.

[47] Xiaoli Ma and Wei Zhang. Performance analysis for MIMO Systems with lattice-reduction aided linear equalization. Communications, IEEE Transactions on, 56(2):309-318, 2008.

[48] Chiao-En Chen. Lattice-reduction-aided MIMO detection under imperfect channel state information. In Acoustics Speech and Signal Processing (ICASSP), 2010 IEEE International Conference on, pages 3446-3449. IEEE, 2010.

[49] Lin Bai, Chen Chen, and Jinho Choi. Lattice reduction aided detection for underdetermined MIMO Systems: A pre-voting cancellation approach. In Vehicular Technology Conference (VTC 2010-Spring), 2010 IEEE 71st, pages 1-5. IEEE, 2010.

[50] Brian Gestner, Wei Zhang, Xiaoli Ma, and David V Anderson. Lattice reduction for MIMO detection: from theoretical analysis to hardware realization. Circuits and Systems I: Regular Papers, IEEE Transactions on, 58(4):813-826, 2011.

[51] Chiao-En Chen, Hang Su, Chun-Fu Liao, and Yuang-Hao Huang. A constant-throughput lll algorithm with deep insertion for lr-aided MIMO detection. In Circuits and Systems (ISCAS), 2012 IEEE International Symposium on, pages 1251-1254. IEEE, 2012.

[52] Jaehyun Park and Joohwan Chun. Efficient lattice-reduction-aided successive interference cancellation for clustered multiuser MIMO System. Vehicular Technology, IEEE Transactions on, 61(8):3643-3655, 2012. 
[53] Qi Zhou and Xiaoli Ma. An improved lr-aided k-best algorithm for MIMO detection. In Wireless Communications $\mathcal{G}$ Signal Processing (WCSP), 2012 International Conference on, pages 1-5. IEEE, 2012.

[54] Hyunsub Kim, Jangyong Park, Hyukyeon Lee, and Jaeseok Kim. Near-ml MIMO detection algorithm with lr-aided fixed-complexity tree searching. Communications Letters, IEEE, 18(12):2221-2224, 2014.

[55] Raul Ambrozio Valente, José Carlos Marinello, and Taufik Abrão. Lr-aided MIMO detectors under correlated and imperfectly estimated channels. Wireless personal communications, 77(1):173-196, 2014.

[56] Shu Fang, Jian Wu, Chengyi Lu, Yuan-chao Han, et al. Simplified qr-decomposition based and lattice reduction-assisted multi-user multiple-input-multiple-output precoding scheme. IET Communications, 10(5):586-593, 2016.

[57] Wei Wang, Meixia Hu, Yongzhao Li, Hailin Zhang, and Zan Li. Computationally efficient fixed complexity 111 algorithm for lattice-reduction-aided multiple-input-multiple-output precoding. IET Communications, 10(17):2328-2335, 2016.

[58] Alex Miyamoto Mussi, Bruno Felipe Costa, and Taufik Abrão. Efficient lattice reduction aided detectors under realistic mimo channels. Wireless Personal Communications, pages 1-32, 2016.

[59] Hengliang Zhu, Behrouz Farhang-Boroujeny, and Rong-Rong Chen. On performance of sphere decoding and markov chain monte carlo detection methods. In Signal Processing Advances in Wireless Communications, 2005 IEEE 6th Workshop on, pages 86-90. IEEE, 2005.

[60] Xuehong Mao, Peiman Amini, and Behrouz Farhang-Boroujeny. Markov chain monte carlo MIMO detection methods for high signal-to-noise ratio regimes. In Global Telecommunications Conference, 200\%. GLOBECOM'0\%. IEEE, pages 3979-3983. IEEE, 2007.

[61] Ronghui Peng, Koon Hoo Teo, Jinyun Zhang, and Rong-Rong Chen. Low-complexity hybrid QRD-MCMC MIMO detection. In Global Telecommunications Conference, 2008. IEEE GLOBECOM 2008. IEEE, pages 1-5. IEEE, 2008.

[62] Ashok Kumar, Suresh Chandrasekaran, Ananthanarayanan Chockalingam, and B Sundar Rajan. Near-optimal large-MIMO detection using randomized MCMC and randomized search algorithms. In Communications (ICC), 2011 IEEE International Conference on, pages 1-5. IEEE, 2011.

[63] Fang-Li Yuan, Chia-Hsiang Yang, and Dejan Marković. A hardware-efficient vlsi architecture for hybrid sphere-MCMC detection. In Global Telecommunications Conference (GLOBECOM 2011), 2011 IEEE, pages 1-6. IEEE, 2011. 
[64] Tanumay Datta, N Ashok Kumar, Ananthanarayanan Chockalingam, and B Sundar Rajan. A novel MCMC based receiver for large-scale uplink multiuser MIMO Systems. arXiv preprint arXiv:1201.6034, 2012.

[65] Jienan Chen, Jianhao Hu, and Gerald E Sobelman. Stochastic iterative mimo detection system: Algorithm and hardware design. IEEE Transactions on Circuits and Systems I: Regular Papers, 62(4):1205-1214, 2015.

[66] Jinho Choi. An mcmc-mimo detector as a stochastic linear system solver using successive overrelexation. IEEE Transactions on Wireless Communications, 15(2):1445-1455, 2016.

[67] Lin Bai, Tian Li, Jianwei Liu, Quan Yu, and Jinho Choi. Large-scale mimo detection using mcmc approach with blockwise sampling. IEEE Transactions on Communications, 64(9):3697-3707, 2016.

[68] Catherine ZW Hassell Sweatman and John S Thompson. Orthotope sphere decoding and parallelotope decodingreduced complexity optimum detection algorithms for MIMO channels. Signal processing, 86(7):1518-1537, 2006.

[69] Giorgio Taricco and Giulio Coluccia. Optimum receiver design for correlated rician fading MIMO channels with pilot-aided detection. Selected Areas in Communications, IEEE Journal on, 25(7):1311-1321, 2007.

[70] Mohamed Chouayakh, Andreas Knopp, and Berthold Lankl. Low-effort near maximum likelihood MIMO detection with optimum hardware resource exploitation. Electronics letters, 43(20):1104-1106, 2007.

[71] Lie-Liang Yang. Using multi-stage mmse detection to approach optimum error performance in multiantenna MIMO Systems. In Vehicular Technology Conference Fall (VTC 2009-Fall), 2009 IEEE 70th, pages 1-5. IEEE, 2009.

[72] Giulio Coluccia, Erwin Riegler, Christoph Mecklenbräuker, and Giorgio Taricco. Optimum MIMO-OFDM detection with pilot-aided channel state information. Selected Topics in Signal Processing, IEEE Journal of, 3(6):1053-1065, 2009.

[73] Ricardo Tadashi Kobayashi, Fernando Ciriaco, and Taufik Abrão. Efficient near-optimum detectors for large mimo systems under correlated channels. Wireless Personal Communications, 83(2):1287-1311, 2015.

[74] Dan Zhang, Luciano Leonel Mendes, Maximilian Matthé, Ivan Simões Gaspar, Nicola Michailow, and Gerhard P Fettweis. Expectation propagation for near-optimum detection of mimo-gfdm signals. IEEE Transactions on Wireless Communications, 15(2):1045-1062, 2016. 
[75] Cai Yueming, Xu Xin, Cheng Yunpeng, Xu Youyun, and Li Zi. A SISO iterative probabilistic data association detector for MIMO Systems. In Communication Technology, 2006. ICCT'06. International Conference on, pages 1-4. IEEE, 2006.

[76] Shaoshi Yang, Tiejun Lv, Xiang Yun, Xinghui Su, and Jinhuan Xia. A probabilistic data association based MIMO detector using joint detection of consecutive symbol vectors. In Communication Systems, 2008. ICCS 2008. 11th IEEE Singapore International Conference on, pages 436-440. IEEE, 2008.

[77] Shaoshi Yang and Tiejun Lv. A novel probabilistic data association based MIMO detector using joint detection of consecutive symbol vectors. In Consumer Communications and Networking Conference, 2009. CCNC 2009. 6th IEEE, pages 1-5. IEEE, 2009.

[78] Shaoshi Yang, Tiejun Lv, and Lajos Hanzo. Unified bit-based probabilistic data association aided MIMO detection for high-order QAM. In Wireless Communications and Networking Conference (WCNC), 2011 IEEE, pages 1629-1634. IEEE, 2011.

[79] Shaoshi Yang and Lajos Hanzo. Exact bayes' theorem based probabilistic data association for iterative MIMO detection and decoding. In Global Communications Conference (GLOBECOM), 2013 IEEE, pages 1891-1896. IEEE, 2013.

[80] Shaoshi Yang, Li Wang, Tiejun Lv, and Lajos Hanzo. Approximate bayesian probabilistic-data-association-aided iterative detection for MIMO Systems using arbitrary-ary modulation. Vehicular Technology, IEEE Transactions on, 62(3):1228-1240, 2013.

[81] Shaoshi Yang, Tiejun Lv, Robert G Maunder, and Lajos Hanzo. From nominal to true a posteriori probabilities: an exact bayesian theorem based probabilistic data association approach for iterative mimo detection and decoding. Communications, IEEE Transactions on, 61(7):2782-2793, 2013.

[82] Yufang Yin, Gangjun Li, and Hua Wei. Distributed probabilistic data association detector with turbo base-station cooperations in multi-user multi-cell mimo systems. EURASIP Journal on Wireless Communications and Networking, 2017(1):81, 2017.

[83] Darryl Dexu Lin and Teng Joon Lim. A variational inference framework for soft-in soft-out detection in multiple-access channels. IEEE Transactions on Information Theory, 55(5):2345-2364, 2009.

[84] Christoph Studer and Helmut Bolcskei. Soft-input soft-output single tree-search sphere decoding. IEEE Transactions on Information Theory, 56(10):4827-4842, 2010.

[85] Mirsad Cirkic and Erik G Larsson. Sumis: Near-optimal soft-in soft-out mimo detection with low and fixed complexity. IEEE Transactions on Signal Processing, 62(12):3084-3097, 2014. 
[86] Wang Yi. An Investigation of Peak-to-Average Power Reduction in MIMO-OFDM Systems. PhD thesis, Blekinge Institute of Technology, 2009.

[87] Jakob Hoydis, Stephan Ten Brink, and Mérouane Debbah. Comparison of linear precoding schemes for downlink massive MIMO. In Communications (ICC), 2012 IEEE International Conference on, pages 2135-2139. IEEE, 2012.

[88] Sinh Le Hong Nguyen and Ali Ghrayeb. Compressive sensing-based channel estimation for massive multiuser MIMO Systems. In Wireless Communications and Networking Conference (WCNC), 2013 IEEE, pages 2890-2895. IEEE, 2013.

[89] Yi Zhu, Lingjia Liu, Anding Wang, Krishna Sayana, and Jianzhong Charlie Zhang. Doa estimation and capacity analysis for 2d active massive MIMO Systems. In Communications (ICC), 2013 IEEE International Conference on, pages 4630-4634. IEEE, 2013.

[90] Hua Zhang, Xinru Zheng, Wei Xu, and Xiaohu You. On massive MIMO performance with semi-orthogonal pilot-assisted channel estimation. EURASIP Journal on Wireless Communications and Networking, 2014(1):1-14, 2014.

[91] Andrew J Duly, Taejoon Kim, David J Love, and James V Krogmeier. Closed-loop beam alignment for massive MIMO channel estimation. Communications Letters, IEEE, 18(8):1439-1442, 2014.

[92] Junjie Yang, Shengli Xie, Xiangyun Zhou, Rong Yu, and Yan Zhang. A semiblind two-way training method for discriminatory channel estimation in MIMO Systems. Communications, IEEE Transactions on, 62(7):2400-2410, 2014.

[93] Negin Shariati, Emil Bjornson, Martin Bengtsson, and Mérouane Debbah. Low-complexity polynomial channel estimation in large-scale MIMO with arbitrary statistics. Selected Topics in Signal Processing, IEEE Journal of, 8(5):815-830, 2014.

[94] Xiongbin Rao and Vincent KN Lau. Distributed compressive csit estimation and feedback for fdd multi-user massive MIMO Systems. Signal Processing, IEEE Transactions on, 62(12):3261-3271, 2014.

[95] Muhammad Ali Raza Anjum. A new approach to linear estimation problem in multi-user massive MIMO Systems. arXiv preprint arXiv:1504.07426, 2015.

[96] Ke Li, Xiaoqin Song, M Omair Ahmad, and MNS Swamy. An improved multicell MMSE channel estimation in a massive MIMO System. International Journal of Antennas and Propagation, 2014, 2014.

[97] Xiongbin Rao, Vincent KN Lau, and Xiangming Kong. CSIT estimation and feedback for FDD multi-user massive MIMO Systems. In Acoustics, Speech and Signal Processing (ICASSP), 2014 IEEE International Conference on, pages 3157-3161. IEEE, 2014. 
[98] Zhen Gao, Chao Zhang, Chengran Dai, and Qian Han. Spectrum-efficiency parametric channel estimation scheme for massive MIMO Systems. In Broadband Multimedia Systems and Broadcasting (BMSB), 2014 IEEE International Symposium on, pages 1-4. IEEE, 2014.

[99] Hien Quoc Ngo and Erik G Larsson. Blind estimation of effective downlink channel gains in massive MIMO. arXiv preprint arXiv:1503.09059, 2015.

[100] Chao-Kai Wen, Shi Jin, Kai-Kit Wong, Jung-Chieh Chen, and Pangan Ting. Channel estimation for massive MIMO using gaussian-mixture bayesian learning. Wireless Communications, IEEE Transactions on, 14(3):1356-1368, 2015.

[101] Mohammed Teeti, Jun Sun, David Gesbert, and Yingzhuang Liu. The impact of physical channel on performance of subspace-based channel estimation in massive MIMO Systems. 2015.

[102] Hongxiang Xie, Feifei Gao, Shun Zhang, and Shi Jin. A simple DFT-aided spatial basis expansion model and channel estimation strategy for massive MIMO Systems. arXiv preprint arXiv:1511.04841, 2015.

[103] Chao-Kai Wen, Chang-Jen Wang, Shi Jin, Kai-Kit Wong, and Pangan Ting. Bayes-optimal joint channel-and-data estimation for massive MIMO with low-precision ADCs. 2015.

[104] Fengyang Xu, Yang Xiao, and Dong Wang. Adaptive semi-blind channel estimation for massive MIMO Systems. In Signal Processing (ICSP), 2014 12th International Conference on, pages 1698-1702. IEEE, 2014.

[105] De Mi, Mehrdad Dianati, and Sami Muhaidat. A novel antenna selection scheme for spatially correlated massive MIMO uplinks with imperfect channel estimation. arXiv preprint arXiv:1403.2902, 2014.

[106] Peng Zhang, Lu Gan, Sumei Sun, and Cong Ling. Atomic norm denoising-based channel estimation for massive multiuser MIMO Systems. In Communications (ICC), 2015 IEEE International Conference on, pages 4564-4569. IEEE, 2015.

[107] Zhen Gao, Linglong Dai, Wei Dai, Byonghyo Shim, and Zhaocheng Wang. Structured compressive sensing-based spatio-temporal joint channel estimation for fdd massive mimo. IEEE Transactions on Communications, 64(2):601-617, 2016.

[108] Chao-Kai Wen, Chang-Jen Wang, Shi Jin, Kai-Kit Wong, and Pangan Ting. Bayes-optimal joint channel-and-data estimation for massive mimo with low-precision adcs. IEEE Transactions on Signal Processing, 64(10):2541-2556, 2016.

[109] Weiyang Xu, Wei Xiang, Yunjian Jia, Youjun Li, and Yi Yang. Downlink performance of massive-mimo systems using evd-based channel estimation. IEEE Transactions on Vehicular Technology, 66(4):3045-3058, 2017. 
[110] Wei Huang, Yongming Huang, Wei Xu, and Luxi Yang. Beam-blocked channel estimation for fdd massive mimo with compressed feedback. IEEE Access, 5:11791-11804, 2017.

[111] Yu Zhang, Dongming Wang, Jiangzhou Wang, and Xiaohu You. Channel estimation for massive mimo-ofdm systems by tracking the joint angle-delay subspace. IEEE Access, 4(10):166-10, 2016.

[112] Xin Xiong, Xiaodong Wang, Xiqi Gao, and Xiaohu You. Beam-domain channel estimation for fdd massive mimo systems with optimal thresholds. IEEE Transactions on Wireless Communications, $16(7): 4669-4682,2017$.

[113] Jun Fang, Xingjian Li, Hongbin Li, and Feifei Gao. Low-rank covariance-assisted downlink training and channel estimation for fdd massive mimo systems. IEEE Transactions on Wireless Communications, 16(3):1935-1947, 2017.

[114] Yiqing Zhou, Tung-Sang Ng, Jiangzhou Wang, Kenichi Higuchi, and Mamoru Sawahashi. OFCDM: a promising broadband wireless access technique. IEEE Communications Magazine, 46(3):38-49, 2008.

[115] Yiqing Zhou and Tung-Sang Ng. Joint iterative detection for multi-code MIMO-OFCDM systems. In Circuits and Systems for Communications, 2008. ICCSC 2008. 4th IEEE International Conference on, pages 434-438. IEEE, 2008.

[116] Yiqing Zhou and Tung-Sang Ng. MIMO-OFCDM systems with joint iterative detection and optimal power allocation. IEEE Transactions on Wireless Communications, 7(12):5504-5516, 2008.

[117] Yiqing Zhou and Tung-Sang Ng. Optimal power allocation for channel estimation in MIMO-OFCDM systems. In Circuits and Systems, 2008. APCCAS 2008. IEEE Asia Pacific Conference on, pages 822-825. IEEE, 2008.

[118] Yiqing Zhou and Tung-Sang Ng. Performance analysis on MIMO-OFCDM systems with multi-code transmission. IEEE Transactions on Wireless Communications, 8(9):4426-4433, 2009.

[119] Yiqing Zhou and Tung-Sang Ng. Performance investigation of MIMO-OFCDM with imperfect channel estimation. In Communications (MICC), 2009 IEEE 9th Malaysia International Conference on, pages 223-228. IEEE, 2009.

[120] Fatima Hussain and Alagan Anpalagan. OFCDM-based small femtocells embedded in OFDM-based macro cellular network. In 2013 IEEE International Conference on Communications Workshops (ICC), pages 1199-1204. IEEE, 2013.

[121] Ardavan Rahimian and Farhad Mehran. RF link budget analysis in urban propagation microcell environment for mobile radio communication systems link planning. In Wireless Communications and Signal Processing (WCSP), 2011 International Conference on, pages 1-5. IEEE, 2011. 
[122] Giuseppe Caire, Nihar Jindal, Mari Kobayashi, and Niranjay Ravindran. Multiuser MIMO achievable rates with downlink training and channel state feedback. Information Theory, IEEE Transactions on, 56(6):2845-2866, 2010.

[123] Tetsuki Taniguchi, Nordin Bin Ramli, and Yoshio Karasawa. Subband adaptive array with reduced pilot signal using maximal ratio combining scheme. In Communications, 2009. ICC'09. IEEE International Conference on, pages 1-6. IEEE, 2009.

[124] Imad H Azzam and Raviraj S Adve. Linear precoding for multiuser MIMO Systems with multiple base stations. In Communications, 2009. ICC'09. IEEE International Conference on, pages 1-6. IEEE, 2009.

[125] Vidit Saxena, Gábor Fodor, and Eleftherios Karipidis. Mitigating pilot contamination by pilot reuse and power control schemes for massive MIMO Systems. In Vehicular Technology Conference (VTC Spring), 2015 IEEE 81st, pages 1-6. IEEE, 2015.

[126] Beatrice Tomasi and Maxime Guillaud. Pilot length optimization for spatially correlated multi-user MIMO channel estimation. In 2015 49th Asilomar Conference on Signals, Systems, and Computers, pages 1237-1241. IEEE, 2015.

[127] Imed Hadj-Kacem, Noura Sellami, Inbar Fijalkow, and Aline Roumy. Joint training interval length and power allocation optimization for MIMO flat fading channels. In Wireless Communication Systems, 2009. ISWCS 2009. 6th International Symposium on, pages 16-20. IEEE, 2009.

[128] Jakob Hoydis, Mari Kobayashi, and Mérouane Debbah. Optimal channel training in uplink network MIMO Systems. Signal Processing, IEEE Transactions on, 59(6):2824-2833, 2011.

[129] Jiyong Pang, Jiandong Li, Linjing Zhao, and Zhuo Lü. Optimal training sequences for frequency-selective MIMO correlated fading channels. In Advanced Information Networking and Applications, $200 \%$. AINA'07. 21st International Conference on, pages 820-824. IEEE, 2007.

[130] Imed Hadj-Kacem, Noura Sellami, Inbar Fijalkow, and Aline Roumy. Training interval length optimization for mimo flat fading channels using decision-directed channel estimation. In Wireless and Mobile Computing, Networking and Communications, 2009. WIMOB 2009. IEEE International Conference on, pages 24-29. IEEE, 2009.

[131] Imed Hadj Kacem, Noura Sellami, Aline Roumy, and Inbar Fijalkow. Training sequence optimization for frequency selective channels with MAP equalization. In Communications, Control and Signal Processing, 2008. ISCCSP 2008. 3rd International Symposium on, pages 532-537. IEEE, 2008.

[132] Xiaoli Ma, Liuqing Yang, and Georgios B Giannakis. Optimal training for MIMO frequency-selective fading channels. IEEE Transactions on Wireless Communications, 4(2):453-466, 2005. 
[133] Li Jiandong, Lu Zhuo, Pang Jiyong, and Chen Liang. The impact of training sequence on the capacity of MIMO system. China Comm, pages 53-57, 2006.

[134] Tadilo Endeshaw Bogale and Long Bao Le. Pilot optimization and channel estimation for multiuser massive MIMO systems. In Information Sciences and Systems (CISS), 2014 48th Annual Conference on, pages 1-6. IEEE, 2014.

[135] Xin Geng, Hanying Hu, Weijia Cui, and Yanan Dun. Optimal pilot design for MIMO OFDM channel estimation. In Signal Processing Systems (ICSPS), 2010 2nd International Conference on, volume 2, pages V2-404. IEEE, 2010.

[136] Jubin Jose, Alexei Ashikhmin, Thomas L Marzetta, and Sriram Vishwanath. Pilot contamination and precoding in multi-cell TDD Systems. Wireless Communications, IEEE Transactions on, 10(8):2640-2651, 2011.

[137] T Lakshmi Narasimhan and Ananthanarayanan Chockalingam. Channel hardening-exploiting message passing (CHEMP) receiver in large MIMO systems. In Wireless Communications and Networking Conference (WCNC), 2014 IEEE, pages 815-820. IEEE, 2014.

[138] Noha Hassan and Xavier Fernando. Massive mimo wireless networks: An overview. Electronics, 6(3):63, 2017.

[139] Syed Junaid Nawaz, Abrar Ahmed, Shurjeel Wyne, Kanapathippillai Cumanan, and Zhiguo Ding. 3-d spatial modeling of network interference in Two-Tier Heterogeneous networks. IEEE Access, 5:24040-24053, 2017.

[140] Jinlin Peng, Hao Tang, Peilin Hong, and Kaiping Xue. Stochastic geometry analysis of energy efficiency in heterogeneous network with sleep control. IEEE Wireless Communications Letters, 2(6):615-618, 2013.

[141] Trung Kien Vu, Mehdi Bennis, Sumudu Samarakoon, Mérouane Debbah, and Matti Latva-aho. Joint load balancing and interference mitigation in $5 \mathrm{G}$ heterogeneous networks. IEEE Transactions on Wireless Communications, 16(9):6032-6046, 2017.

[142] Kyuho Son, Eunsung Oh, and Bhaskar Krishnamachari. Energy-aware hierarchical cell configuration: from deployment to operation. In IEEE INFOCOM, pages 289-294, 2011.

[143] Cemil Can Coskun and Ender Ayanoglu. Energy-efficient base station deployment in Heterogeneous Networks. IEEE Wireless Commun. Letters, 3(6):593-596, 2014.

[144] Tiankui Zhang, Jiaojiao Zhao, Lu An, and Dantong Liu. Energy efficiency of base station deployment in ultra dense HetNets: A stochastic geometry analysis. IEEE Wireless Communications Letters, $5(2): 184-187,2016$. 
[145] Tuong Xuan Tran and Kah Chan Teh. Performance analysis of two-tier hetnets with massive MIMO and nonuniformly small cell deployment. IEEE Transactions on Vehicular Technology, 66(11):10044-10054, 2017.

[146] Yong Sheng Soh, Tony QS Quek, Marios Kountouris, and Hyundong Shin. Energy efficient heterogeneous cellular networks. IEEE Journal on Selected Areas in Communications, 31(5):840-850, 2013.

[147] He Zhuang and Tomoaki Ohtsuki. A model based on poisson point process for analyzing MIMO heterogeneous networks utilizing fractional frequency reuse. IEEE Transactions on Wireless Communications, 13(12):6839-6850, 2014.

[148] Raul Hernandez-Aquino, Syed Ali Raza Zaidi, Des McLernon, and Mounir Ghogho. Energy efficiency analysis of two-tier MIMO diversity schemes in poisson cellular networks. IEEE Transactions on Communications, 63(10):3898-3911, 2015.

[149] Chang Li, Jun Zhang, Jeffrey G Andrews, and Khaled B Letaief. Success probability and area spectral efficiency in multiuser MIMO HetNets. IEEE Transactions on Communications, 64(4):1544-1556, 2016.

[150] Marco Di Renzo and Peng Guan. A mathematical framework to the computation of the error probability of downlink MIMO cellular networks by using stochastic geometry. IEEE Transactions on Communications, 62(8):2860-2879, 2014.

[151] Siavash Bayat, Raymond HY Louie, Zhu Han, Branka Vucetic, and Yonghui Li. Distributed user association and Femtocell allocation in heterogeneous wireless networks. IEEE Transactions on Communications, 62(8):3027-3043, 2014.

[152] Seungseob Lee, SuKyoung Lee, Kyungsoo Kim, David Griffith, and Nada Golmie. Optimal deployment of Pico base stations in LTE-advanced heterogeneous networks. Computer Networks, 72:127-139, 2014.

[153] Anabel Martínez-Vargas and Ángel G Andrade. Deployment analysis and optimization of heterogeneous networks under the spectrum underlay strategy. EURASIP Journal on Wireless Communications and Networking, 2015(1):55, 2015.

[154] Robert W Heath, Marios Kountouris, and Tianyang Bai. Modeling heterogeneous network interference using poisson point processes. IEEE Transactions on Signal Processing, 61(16):4114-4126, 2013.

[155] Harpreet S Dhillon, Marios Kountouris, and Jeffrey G Andrews. Downlink mimo hetnets: Modeling, ordering results and performance analysis. IEEE Transactions on Wireless Communications, 12(10):5208-5222, 2013.

[156] Tadilo Endeshaw Bogale and Long Bao Le. Massive MIMO and millimeter wave for 5G wireless hetnet: Potentials and challenges. arXiv preprint arXiv:1510.06359, 2015. 
[157] Junbo Wang, Song Guo, Zixue Cheng, Peng Li, and Jie Wu. Optimization of deployable base stations with guaranteed QOE in disaster scenarios. IEEE Transactions on Vehicular Technology, 66(7), 2017.

[158] Nusrat Mehajabin, Md Abdur Razzaque, Mohammad Mehedi Hassan, Ahmad Almogren, and Atif Alamri. Energy-sustainable relay node deployment in wireless sensor networks. Computer Networks, 104:108-121, 2016.

[159] Punyasha Chatterjee, Sasthi C Ghosh, and Nabanita Das. Load balanced coverage with graded node deployment in wireless sensor networks. IEEE Transactions on Multi-Scale Computing Systems, 3(2):100-112, 2017.

[160] Xuxun Liu. Node deployment based on extra path creation for wireless sensor networks on mountain roads. IEEE Communications Letters, 21(11):2376-2379, 2017.

[161] Wei Kuang Lai and Chung-Shuo Fan. Novel node deployment strategies in corona structure for wireless sensor networks. IEEE Access, 5:3889-3899, 2017.

[162] Yihui Li, Gaoxi Xiao, Gurpreet Singh, and Rashmi Gupta. Algorithms for finding best locations of cluster heads for minimizing energy consumption in wireless sensor networks. Wireless networks, 19(7):1755-1768, 2013.

[163] Yi Shi, Y Thomas Hou, and Alon Efrat. Algorithm design for a class of base station location problems in sensor networks. Wireless Networks, 15(1):21-38, 2009.

[164] Sung Nok Chiu, Dietrich Stoyan, Wilfrid S Kendall, and Joseph Mecke. Stochastic geometry and its applications. John Wiley \& Sons, 2013.

[165] Serkan Ak, Hazer Inaltekin, and H Vincent Poor. Gaussian approximation for the downlink interference in heterogeneous cellular networks. In Information Theory (ISIT), 2016 IEEE International Symposium on, pages 1611-1615. IEEE, 2016.

[166] Keith Conrad. The gaussian integral. University of Connecticut, pages 1-2, 2016.

[167] Harpreet S Dhillon, Radha Krishna Ganti, Francois Baccelli, and Jeffrey G Andrews. Modeling and analysis of k-tier downlink heterogeneous cellular networks. IEEE Journal on Selected Areas in Communications, 30(3):550-560, 2012.

[168] Sarabjot Singh, Harpreet S Dhillon, and Jeffrey G Andrews. Offloading in heterogeneous networks: Modeling, analysis, and design insights. IEEE Transactions on Wireless Communications, 12(5):2484-2497, 2013.

[169] Serge Dubuc. An approximation of the gamma function. Journal of mathematical analysis and applications, 146(2):461-468, 1990. 
[170] Thomas F Coleman and Yuying Li. On the convergence of interior-reflective newton methods for nonlinear minimization subject to bounds. Mathematical programming, 67(1-3):189-224, 1994.

[171] Thomas F Coleman and Yuying Li. An interior trust region approach for nonlinear minimization subject to bounds. SIAM Journal on optimization, 6(2):418-445, 1996.

[172] Mary Ann Branch, Thomas F Coleman, and Yuying Li. A subspace, interior, and conjugate gradient method for large-scale bound-constrained minimization problems. SIAM Journal on Scientific Computing, 21(1):1-23, 1999.

[173] Seungseob Lee, SuKyoung Lee, Kyungsoo Kim, and Yoon Hyuk Kim. Base station placement algorithm for large-scale LTE heterogeneous networks. PloS one, 10(10):e0139190, 2015.

[174] Eitan Altman, Anurag Kumar, Chandramani Singh, and Rajesh Sundaresan. Spatial SINR games of base station placement and mobile association. IEEE/ACM Transactions on Networking (TON), 20(6):1856-1869, 2012.

[175] Shokri Z Selim, Yasser A Almoghathawi, and Mansour Aldajani. Optimal base stations location and configuration for cellular mobile networks. Wireless Networks, 21(1):13-19, 2015.

[176] Mudassar Ali, Shahid Mumtaz, Saad Qaisar, and Muhammad Naeem. Smart heterogeneous networks: a 5G paradigm. Telecommunication Systems, 66(2):311-330, 2017.

[177] Tianqing Zhou, Zunxiong Liu, Dong Qin, Nan Jiang, and Chunguo Li. User association with maximizing weighted sum energy efficiency for massive MIMO-Enabled Heterogeneous cellular networks. IEEE Communications Letters, 21(10):2250-2253, 2017.

[178] Yi Xu and Shiwen Mao. User association in massive mimo hetnets. IEEE Systems Journal, 11(1):7-19, 2017.

[179] Javier Rubio, Antonio Pascual-Iserte, Jaume del Olmo, and Josep Vidal. User association strategies in HetNets leading to rate balancing under energy constraints. EURASIP Journal on Wireless Communications and Networking, 2017(1):204, 2017.

[180] Qiaoyang Ye, Beiyu Rong, Yudong Chen, Mazin Al-Shalash, Constantine Caramanis, and Jeffrey G Andrews. User association for load balancing in heterogeneous cellular networks. IEEE Transactions on Wireless Communications, 12(6):2706-2716, 2013.

[181] Qiaoyang Ye, Ozgun Yilmaz Bursalioglu, Haralabos C Papadopoulos, Constantine Caramanis, and Jeffrey G Andrews. User association and interference management in massive MIMO HetNets. IEEE Transactions on Communications, 64(5):2049-2065, 2016. 
[182] Han-Shin Jo, Young Jin Sang, Ping Xia, and Jeffrey G Andrews. Heterogeneous cellular networks with flexible cell association: A comprehensive downlink SINR analysis. IEEE Transactions on Wireless Communications, 11(10):3484-3495, 2012.

[183] Supratim Deb, Pantelis Monogioudis, Jerzy Miernik, and James P Seymour. Algorithms for enhanced inter-cell interference coordination (eicic) in LTE HetNets. IEEE/ACM transactions on networking, 22(1):137-150, 2014.

[184] Fancheng Kong, Xinghua Sun, and Hongbo Zhu. Optimal biased association scheme with heterogeneous user distribution in HetNets. Wireless Personal Communications, 90(2):575-594, 2016.

[185] Xuefang Nie, Yang Wang, Jiliang Zhang, and Liqin Ding. Coverage and association bias analysis for backhaul constrained HetNets with eICIC and CRE. Wireless Personal Communications, 97(4):4981-5002, 2017.

[186] Fancheng Kong, Xinghua Sun, Victor CM Leung, and Hongbo Zhu. Delay-optimal biased user association in heterogeneous networks. IEEE Transactions on Vehicular Technology, 66(8):7360-7371, 2017.

[187] Evsen Yanmaz and Ozan K Tonguz. Dynamic load balancing and sharing performance of integrated wireless networks. IEEE Journal on Selected Areas in Communications, 22(5):862-872, 2004.

[188] Ziaul Haq Abbas, Fazal Muhammad, and Jiao Lei. Analysis of load balancing and interference management in heterogeneous cellular networks. 2017.

[189] Fazal Muhammad, Ziaul Haq Abbas, and Frank Y Li. Cell association with load balancing in nonuniform heterogeneous cellular networks: Coverage probability and rate analysis. IEEE Transactions on Vehicular Technology, 66(6):5241-5255, 2017.

[190] Subramanian Vasudevan, Rahul N Pupala, and Kathiravetpillai Sivanesan. Dynamic eICICA proactive strategy for improving spectral efficiencies of heterogeneous LTE cellular networks by leveraging user mobility and traffic dynamics. IEEE Transactions on Wireless Communications, 12(10):4956-4969, 2013.

[191] Yicheng Lin, Wei Bao, Wei Yu, and Ben Liang. Optimizing user association and spectrum allocation in HetNets: A utility perspective. IEEE Journal on Selected Areas in Communications, 33(6):1025-1039, 2015.

[192] Yulin Jia, Ming Zhao, and Wuyang Zhou. Joint user association and eICIC for max-min fairness in HetNets. IEEE Communications Letters, 20(3):546-549, 2016.

[193] Hao Zhou, Yusheng Ji, Xiaoyan Wang, and Shigeki Yamada. eICIC configuration algorithm with service scalability in heterogeneous cellular networks. IEEE/ACM Transactions on Networking (TON), 25(1):520-535, 2017. 
[194] Jie Zheng, Jiandong Li, Nannan Wang, and Xiaoniu Yang. Joint load balancing of downlink and uplink for eICIC in heterogeneous network. IEEE Transactions on Vehicular Technology, 66(7):6388-6398, 2017.

[195] Jeffrey G Andrews, François Baccelli, and Radha Krishna Ganti. A tractable approach to coverage and rate in cellular networks. IEEE Transactions on communications, 59(11):3122-3134, 2011. 